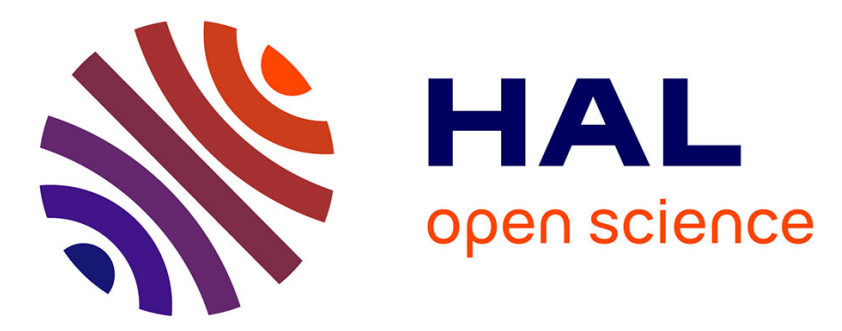

\title{
Les puits et fosses de la colline Saint-Jacques à Cavaillon (Vaucluse)
}

André Dumoulin

\section{To cite this version:}

André Dumoulin. Les puits et fosses de la colline Saint-Jacques à Cavaillon (Vaucluse). Gallia - Fouilles et monuments archéologiques en France métropolitaine, 1965, 23 (1), pp.1-85. 10.3406/galia.1965.2219 . hal-01933651

\section{HAL Id: hal-01933651 \\ https://hal.science/hal-01933651}

Submitted on 26 Feb 2020

HAL is a multi-disciplinary open access archive for the deposit and dissemination of scientific research documents, whether they are published or not. The documents may come from teaching and research institutions in France or abroad, or from public or private research centers.
L'archive ouverte pluridisciplinaire HAL, est destinée au dépôt et à la diffusion de documents scientifiques de niveau recherche, publiés ou non, émanant des établissements d'enseignement et de recherche français ou étrangers, des laboratoires publics ou privés.

\section{(이) $\$$}

Distributed under a Creative Commons Attribution - NonCommercial - NoDerivatives $\mid 4.0$ 


\title{
LES PUITS ET FOSSES DE LA COLLINE SAINT-JACQUES A CAVAILLON (VAUCLUSE)
}

\author{
par André DUMOULIN
}

La colline saint-Jacpues, ancien oppidum de la tribu gauloise des Carares, est un petit massif rétace qui emerge de la plaine quaternaire formée par les alluvions de la I) urane el de son aflluent le Coulon. Sur le plateau qui en couronne le sommel (alt. $190 \mathrm{~m}$.) et sur se's pentes, de nombreuse's monnairs massaliotes ont été décourertes de lous temps ${ }^{1}$ aver des fragments de polerie el des filuules se rapportant surtout à l'epoque de la Tène2. Des fonds de rabanes creusés dans le rocher, ainsi que des restes de rempart, sont visibles en plusieurs endroits, of si les piétons acceident aujourd'hui au sommet par un escalier rustique construit au $\mathrm{x}^{\mathrm{r}} \mathrm{e}$ siecle, les automobilistes empruntent une route morlerne qui suil le méme vallon que l'ancien el unique chemin primitif appele encore : la voie romaine. Cette voie, dont on distingue toujours le tracé, escaladait en pente douce, par le nord, la seule partie de la colline abordable naturellement, les autres versants étant, soit terminés par des falaises abruptes, soit par des vallons descendlant vers la Duranee qui longe de massif au sud ( lig. 1).

Au quartier dit des lergers, less rouches de l'l rgonien supérieur qui constituent l'ossalure de la colline saint-.Jacques, inclinées vers le nord, s'ennoient sous une nappe importante de graviers formés par des éboulis de pente mélangés à des apports éoliens, et à des galets provenant des alluvions de la Durane et du Coulon ${ }^{3}$. Ces graviers sont utilisés dans te Bàtiment. Trois carrieres d'emprunt s'écletonnent en bordure de la R. X. 573, dite "route d'A vignon ", à sa sortie ouest de la ville de Ciavaillon. C'est au cours de leur exploi-

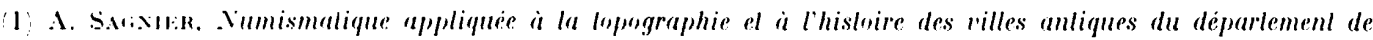

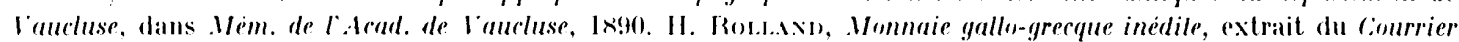

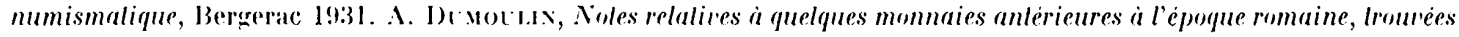

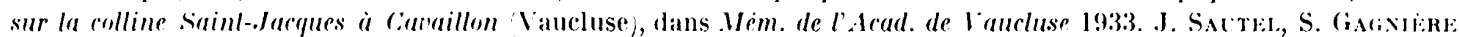
et I. Groman, Essais historiques sur le diparlement de Vaucluse, Prehistoire el Protohistoire, 1.yon, Rey 1933, p. 135, nos 75 at at 757 en note).

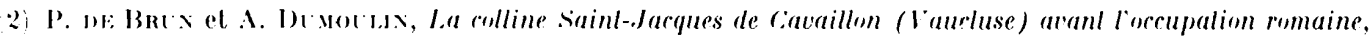

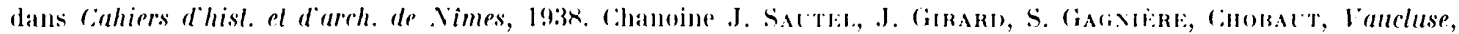
Mistrire locale. Avignon, Bulliere, 1941, 1. \$1, fig. 57.

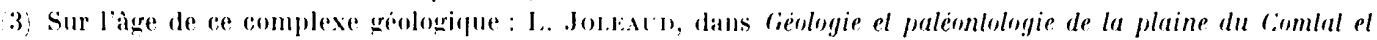

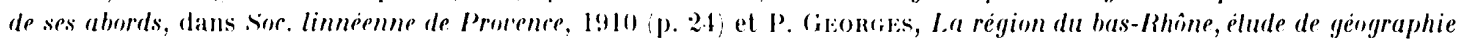
regionale, Paris, Bailliere, 1935 p. 114, Ce dernier auteur le date de l'epoque chellenne. 


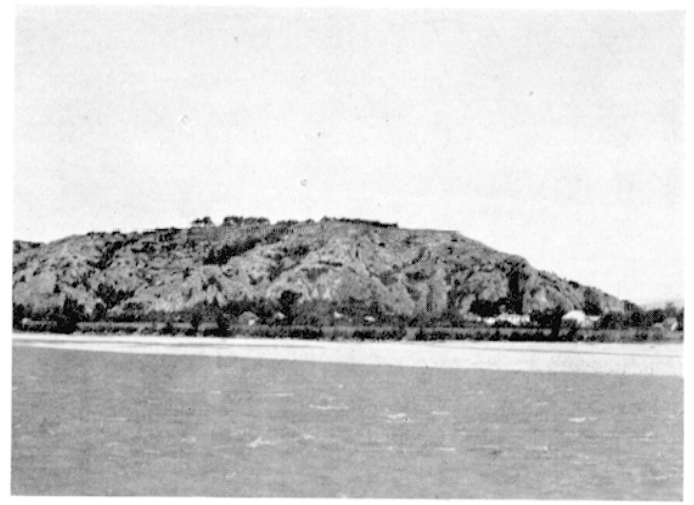

1. Ja colline Saint-Jacepues. de Cavaillon, vue du versanl de la Domance.

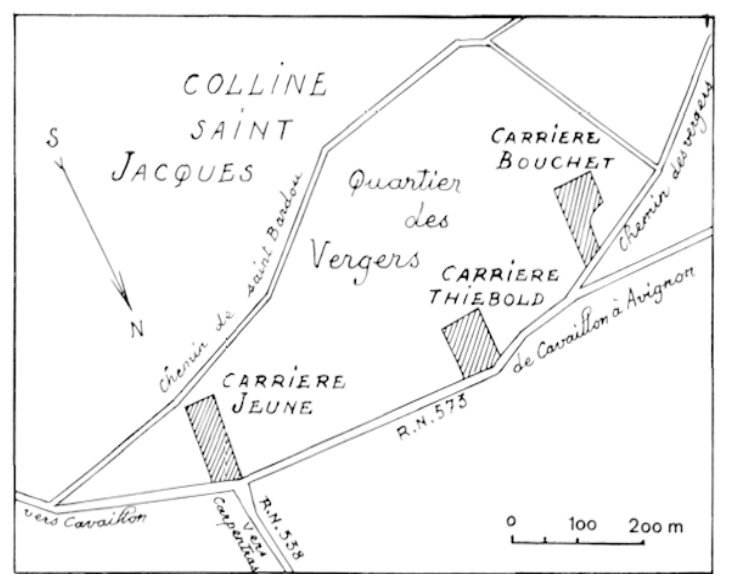

Lation, le plus souvent, malheureusement. a la suite de coups de mines, que des excavalions reusers anciennement dans ces graviers ont ete a rliverses reprises mises au jour. les premières en 19\%6 dans la graviere de .I. A. Jeune (parrelles 67), 671. section H dite des Iscles. du radastre); ensuite an 1941 dans relle de .I. A.

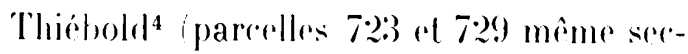
tion: à $400 \mathrm{~m}$. à l'ouest de la préerelente; enfin an 1948 une nouvelle carrière ouverte a list in. a l'ouest de celle de Thiobold

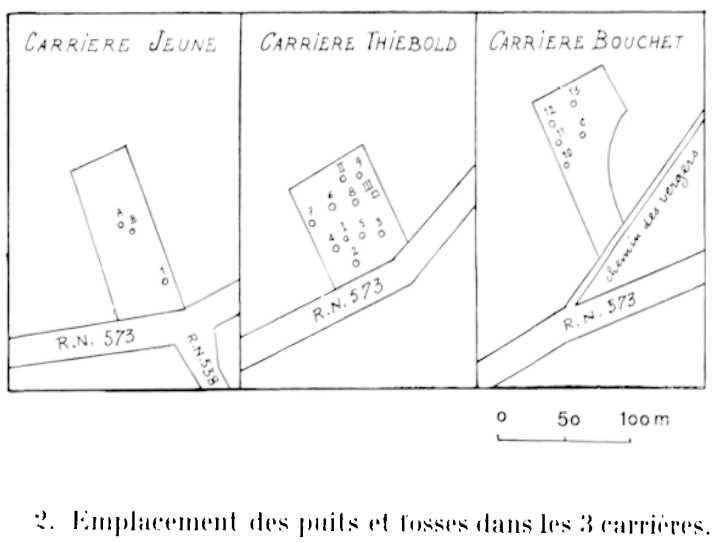

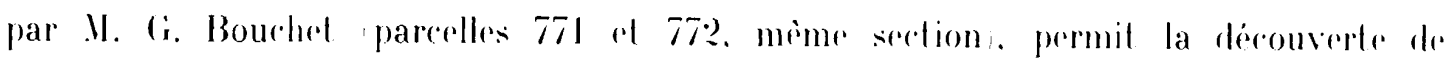
plusieurs autres puils, en partieulier en lys) lors d'une exploitation intensive (fig. : : 5 .

lies excavalions sont dre trois sortes:

$1^{\circ}$ bes puits proprement dits. en majorite nous en dérirons 13 , qui anaient cte fores vertiralement dans l'épaisseur du gravier jusqu'i la nappe aquifire. sur une hauteur de ló mil res environ. l'un diamilre variant de $0.810 \mathrm{~m}$. a $1.70 \mathrm{~m}$. irrégulierement arrondis. ces puits se presentaient en coupe sur le front de la carriere creusis a mème le gravier, sans aurun revelement intérieur. Ja plus souvent la partie supérieure manquait, mais nous avons pu savoir que leur orifice alail surmonte d'une petile murelle circulaire. Cormée de trois ou qualre rangées de pierres serdes. sur laquelle reposail une margelle rarré faile de deux parties symélriques juxlaposées creusésesur le dessus de

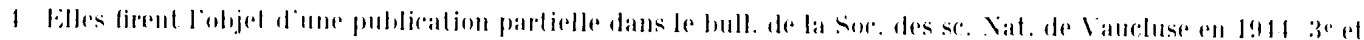

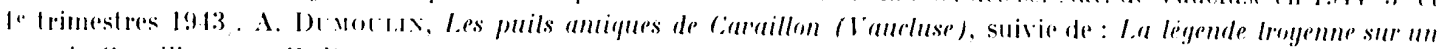

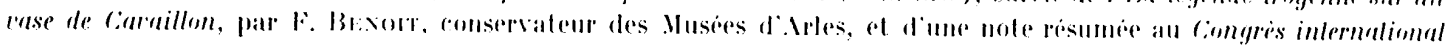

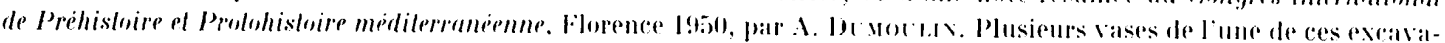

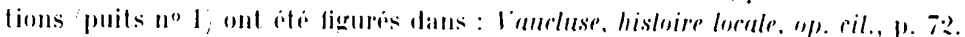

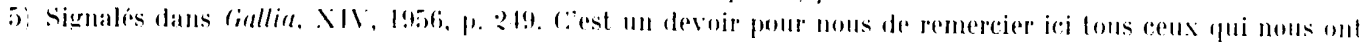

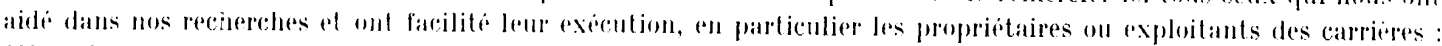

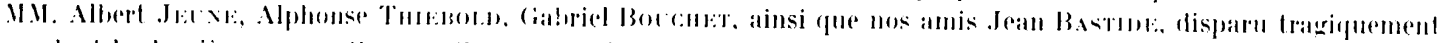

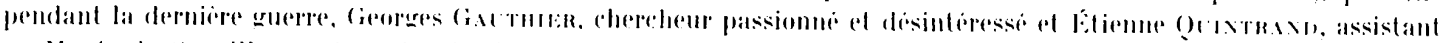
all Musere de lavaillom, anteur des dessins qui illustrent notre travail. 


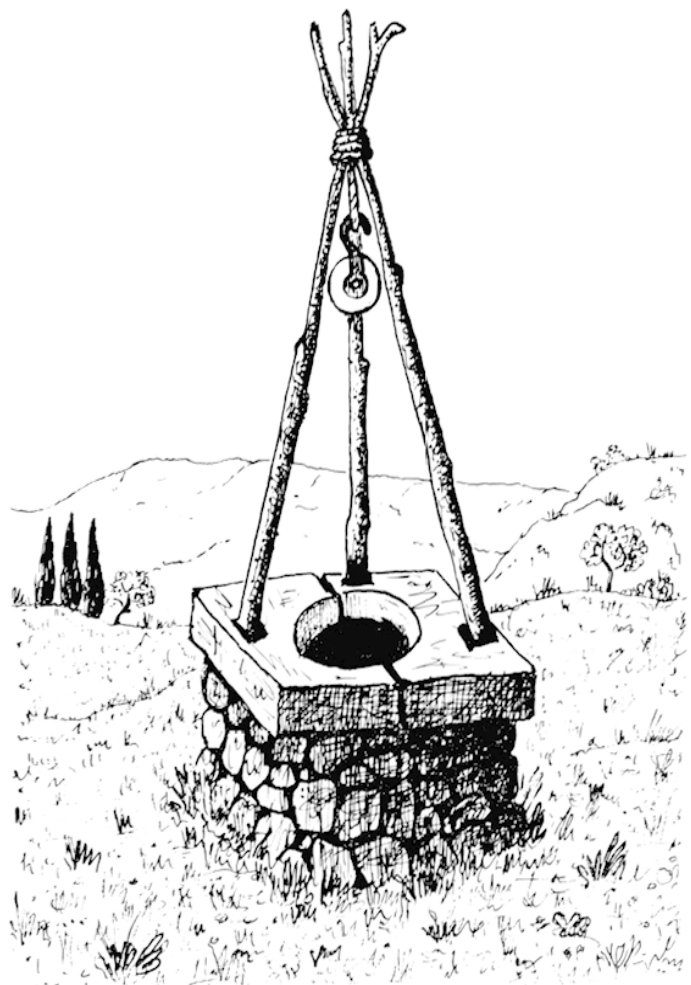

3. Orifice diun puits of sa marerelle reconstitution.

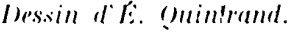

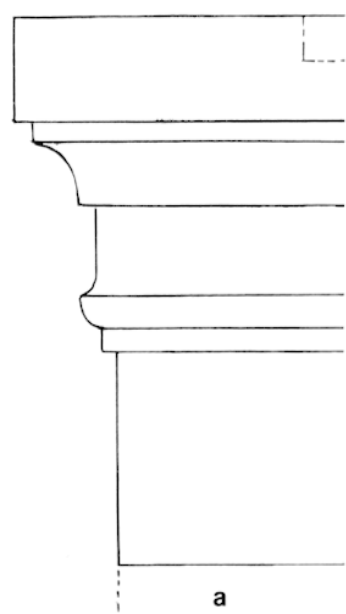

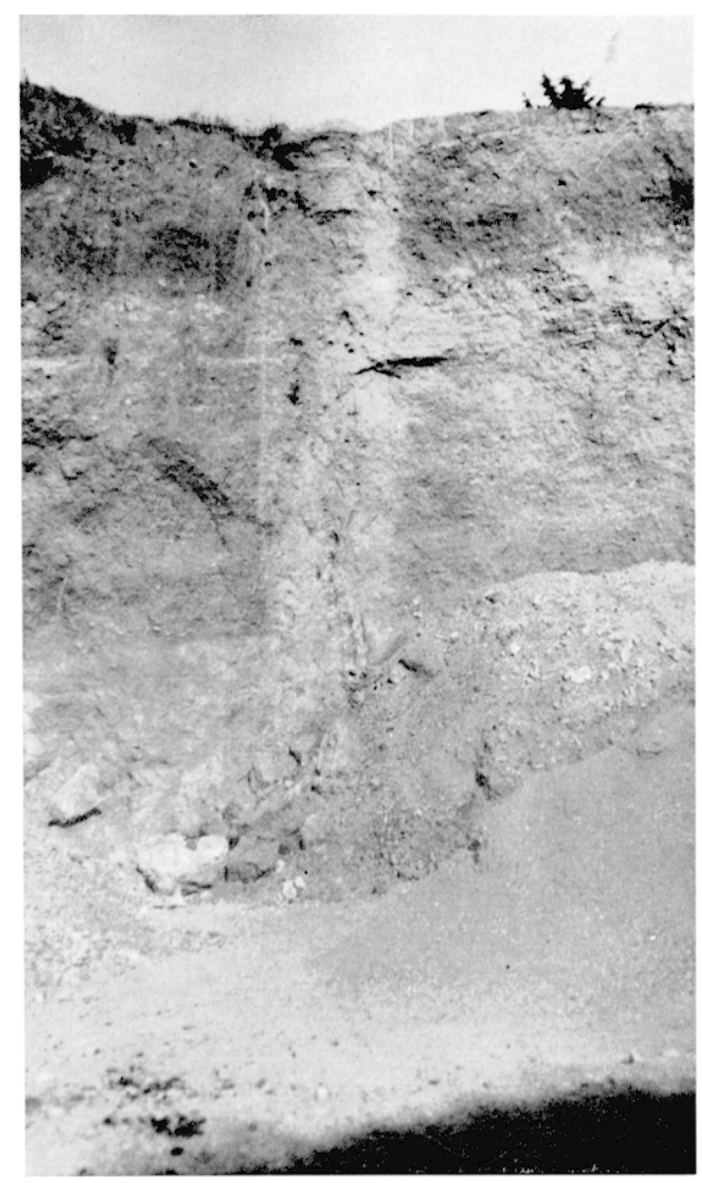

4. Le puits no 7 lors de sa decouverte. On voit, (en haut, lun des chapiteaux de colonne qui en obstruaient liorifice.

Irois pelites encoches reclangulaires pour l'appui des montants supportant la poulie (fig. 3). I)ans la plupart des cas. ces puit élaient comblés sur toute leur hauleur par une terre arqulo-sableuse conlenant une grande quanlilé de vases brisés, mélangés à des ossements d'animaux. dechels de ruisine. cendre el charbons. et décombres principalement dans leur partie supérieure. (Guelques-uns. par exception. furent découverts entierement vides. et on voyait encore l'eau qui miroitait au fond.

$2^{\circ}$ Ies fosses profondes (au nombre de lrois) : sortes de galeries verticales rylindriques. (une seule. la fosse $n^{0}$ :3 éail de section horizontale carrée assez semblahles aux puils. mais dont le creusement avait éte arrile volontairement a une profondeur de quelquess mitres. n'atleignanl jamais l'eau. C'élaient des silos a provisions ou parde-manger.

$3^{\circ}$ Des fosses peu profondes (trois également), à usage sépulcral. 
L'énorme matériel céramique que nous avons recueilli (plus de 30.000 tessons) a nécessité un travail considérable de nettoyage, classement et restauration, qui a demandé plusieurs années. Ainsi, près de 200 récipients ont pu ètre reconstitués, donnant un éventail à peu près complet de la vaisselle en usage chez les habitants primitifs de l'antique Cabellio. Une salle entière du .Iusée de Cavaillon leur a été consacrée. I)ans la réserve, 72 caisses contiennent les fragments d'environ 300 ) autres vases, incomplets, de même provenance.

\section{Les puits}

Sur 13, nous n'avons pu en fouiller entièrement que 4 (puits nos $7,5,10$ et 13), dont l'emplacement ne gênait ni le travail des carriers ni le passage des véhicules. Pour les autres, nous avons dù nous contenter de passer au crible la colonne de terre à hauteur du front d'exploitation, soit 5 m. environ, éboulée par les coups de mine, Nous leur avons donné un numéro d'ordre chronologique mais nous décrirons en premier lieu le puits no 7 le plus important de tous.

\section{PUITs $\times 07$}

IIis au jour dans la carrière Thiébold en 1945, il n'a été déblayé qu'en 1947 (fig: 4 j. Profondeur totale : $15 \mathrm{~m}$. (5 depuis l'ouverture jusqu'au niveau de base d'exploitation de la gravière, 10 en dessous jusqu'à l'eau). Sensiblement cylindrique (diametre: $1,10 \mathrm{~m}$.), il s'élargissait à sa partie inférieure et formait voùte au contact de la nappe aquifere. Son orifice, dont la partie supérieure et la margelle avaient disparu, sans doute lors de l'enlívement de la "découverte" ${ }^{6}$, était "bouché " par 2 grands chapitraux de colonne" less éléments architecturaux se rapprochent tous deux de l'orrlre toscan. Le premier qui est en mollasse calcaire burdigalienne jaune clair, des Taillades, a ses moulures très érodées: sous un tailloir carré mortaisé, échine en cavet droit surmontant un gorgerin terminé par un astragale en quart de rond droit. Cine portion de colonne lisse de 50 $\mathrm{cm}$. de diametre faisant corps avec le chapiteau, le prolonge de $21,50 \mathrm{~cm}$. (fig. 5) a).

Le 2e chapiteau, en mollasse blanche d'Oppède, comprend un tailloir carré mortaisé comme le premier, une échine en doucine droite et un gorgerin entre deux astragales, celui du haut en cavet droit, celui du bas en quart de rond renversé, ( fig. j) b). La terre de remplissage contenait une grande quantité de tessons mélangés à des déchets de cuisine et des décombres à sa partie supérieure : enduits muraux, blocs de mortier, moellons.

Il convient de distinguer 2 couches : l'une supérieure, de $4 \mathrm{~m}$., rlu i ${ }^{\mathrm{e}}$ s. ap. I.-C., l'autre inféricure, de $11 \mathrm{~m}$. du I ${ }^{\text {er }}$ siècle avant.

(6) J.es carriers appellent ainsi la couche de terre végétale qui recouvre directement le gravier, 50 à $60 \mathrm{~cm}$. environ. Ils ont soin de l'enlever sur une large surface, avant de commencer l'exploitation, a fin quicelle ne se mélange pas avec le gravier sous-jacent.

(7) Des chapiteanx et füts de colonnes ont eté trouvés également dans 2 puits du site d'Alésia (Citcd'Or puits Nos 3 et 6. Voir J. Tortan, Vole sur les puils décomerts à Alesia en 1906, Bull. arch., p. 163 et 165 . 

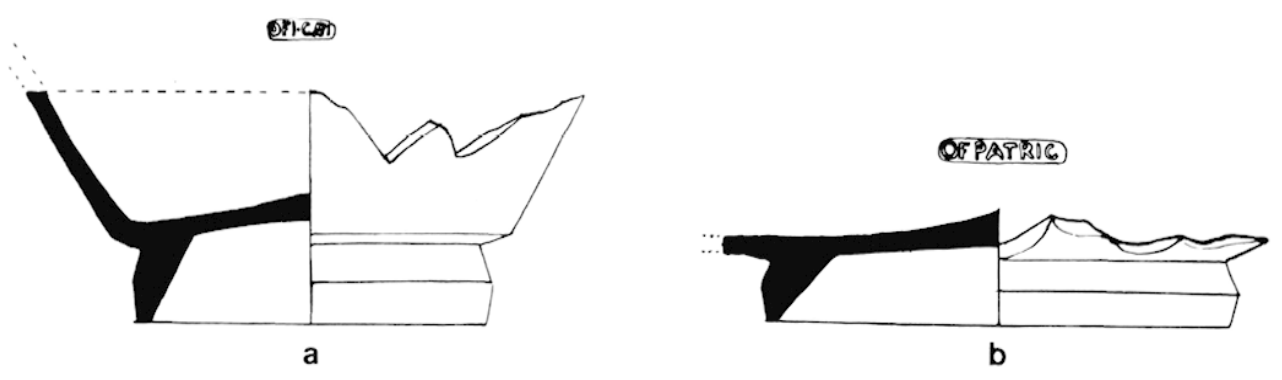

(4a)
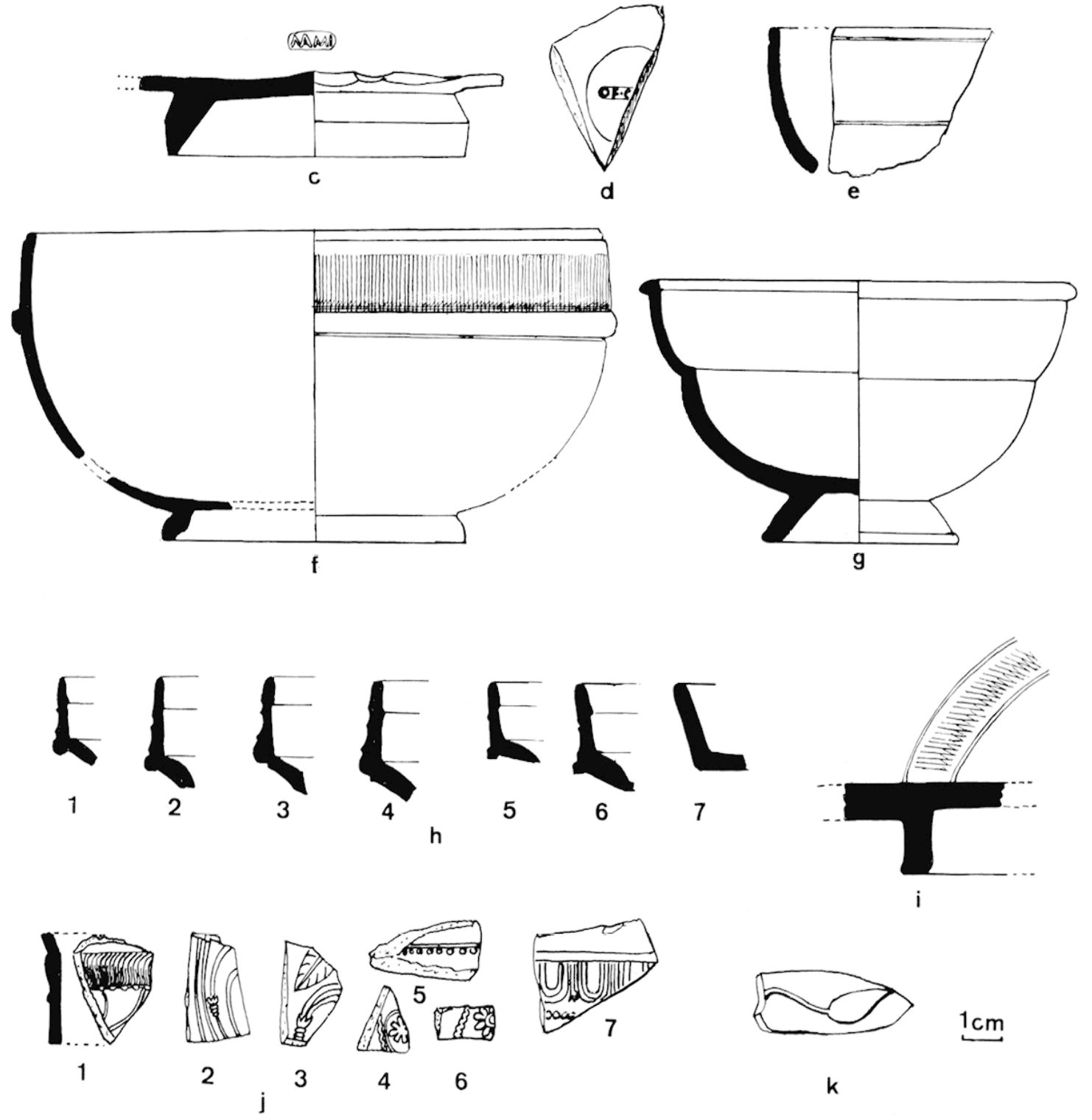

6. Puits no 7 . Poterie sigrille de la couche supérieure. 


\section{Couche supérieure}

Polerie fine. - 1. Terra sigillata :

a) Vases lisses : Ene trentaine de fragments de bols tronconiques (f. I)rag. 33) appartenant ì jécipients différents. dont un fond signé OFI.C.XNTI (IN et T liés) (fig. 6 a) : Canlus potier de la Graufesenque, rigne de Tibere-Claude ${ }^{8}$. Fond d'assiette marqué OFP. ITRIC (fig. (; b) : I'alricius. la Ciraufesenque, période Néron-Domitien ${ }^{9}$. Fond d'assietle à beau vernis rouge carminé estampillé M.IMI (Met I liés) (fig. 6 c.) : Mammius atelier de Lezoux, période Trajan- Intonin ${ }^{10}$. Petil fragnment de fond de bol avec 0. F. C.... (fig. $6 \mathrm{~d}$ ). Bol à hachures verticales le long du bord (f. I)rag. 2425) (fig. $6 \mathrm{f}$ ), époque de Claude ${ }^{11}$. Bol à panse à 2 bourrelets (f. Drag. 27) (fig. $6 \mathrm{~g}$ ), ćpoque de claude ${ }^{12}$. Fragment de coupe f. Ritt. 8 (fig. 6 e). 18 fragments d'assiettes à bord de profils divers (f. I)rag. 15) 17 ) (fig. 6 h 1 a 7 ) appartenant a 7 récipients différents, époque de claude ${ }^{13}$ et 3 fragments de fonds guilloches (fig. 6 i). 2 pieds de vases imitation sigillée, rouge mat.

b) Vases ornés : ils se résument à 6 petils tessons de bol. f. Drag. 29, avec rinceaux, rosares, ef ligne perlée (fig. 6 j. 1 a 6 ), 1 fragment de bol f. Drag. 37 à rangée d'oves (fig. 6 j, 7 ), et 4 fragments. de bords d'écuelles barbotinées (fig. $6 \mathrm{k}$ ), d'époque flavienne.

B. Vases à parois minces:

a) Petit pot à bord court évasé, panse large el base étroite, anse arrondie verticale. en arcrile fine à vernis orangé brillant $(1 \mathrm{H} .: 6 \mathrm{~cm}, 8)$ (fig. $7 \mathrm{a}$ ).

b) Fragments de parois de vases ornés d'écailles de pommes de pin (fig. 7 b) (époque ClaudeXéron ${ }^{14}$, de ponctuation quadrillée en relief (fig. $7 \mathrm{c}$ ), de perles, arceaux et batonnets obliques: (fig. 7 d), panse à décor mamillaire (époque Claude-Xéron) (fig. 7 e); bord de coupe ì paroi granuleuse. par adjonction de sable dans le vernis (époque Tibere-Claude) (fig. $7 \mathrm{f}$ ).

II. Polerie commune. - Crne en terre grise, panse qalbée, fond plat $(1 \mathrm{H} .: 26 \mathrm{~cm})(\mathrm{fig} .8$ a). Crne à panse striéc horizontalement (II. :22 cm) (fig. 8 b). Lrne en terre grise a lives arrondies, col oblique à épaulement (H. : $\left.20 \mathrm{~cm}(\mathrm{fig} .8 \mathrm{c})^{15}\right)$. Cne rentaine de tessons de poterie gris-fer, dure, appartenant : a 2 vases à liquide à bec trilobé, une marmite et plusieurs urnes lisses de forme courante.

III. Divers. - - jéléments de corniche et de revêtement en mollasse blanche moulurée, fragrments de peinture murale polychrome, de plaques d'argile de clayonnage, blors de morlier de chaux et décombres.

\section{Couche inferieure}

Sur 11 mètres d'épaisseur jusqu'à la couche aquifère, le contenu de ce puits présentail une grande homogéniété de types de céramique que nous allons décrire en détail. Signalons d'abord la présence de 2 pièces de monnaie trouvées au même niveau, a $8 \mathrm{~m}$. de profondeur :

$1^{\circ}$ Quinaire (arg.) de Caius Egnaluleius (101 av. J.-(.) : Avers C(aius) E(NATVLEI(us) (ANT et $\mathrm{V}$ et $\mathrm{L}$. liés), C(aii) F(ilius). Tête laurée d'Apollon à dr. Dans le champ : Q(uinarius). -..

(8) F. Oswald, Inder of Pollers' Slamps on Terra sigillata, 1931, p. is.

(9) Ibid., p. 233.

(10) Ibid., p. 181 .

(11) IP. Oswat.d and D. Pryce, An Inlrodurtion to the Sludy of Terra sigillala, 1920, p. xi., $1^{0 .} 3$ et 4.

(12) Ibid., pl. XLIX, no5.

(13) Ibid., pl. XLII, no $18,19,20$.

14. Type d'ornementation représenté dans la nécropole de Vintimille : Nino Lasisocila, Riv. Ingauna $e$ Inlemelia, IV, 1938, no 207 tombe 152.

(15) Forme similaire rencontrée dans plusieurs tombes d'une nécropole gallo-romaine a incincration à Ampurias (Espagne) datie du ler quart. du $1^{\text {er }}$ siecle ap. J.-C: : Martin Amagno, Las necropolis de Ampurias, 1955, II, in fine, planche des urnes, type $V I$ 'inc. Rubert nos 36 et 6 ). 

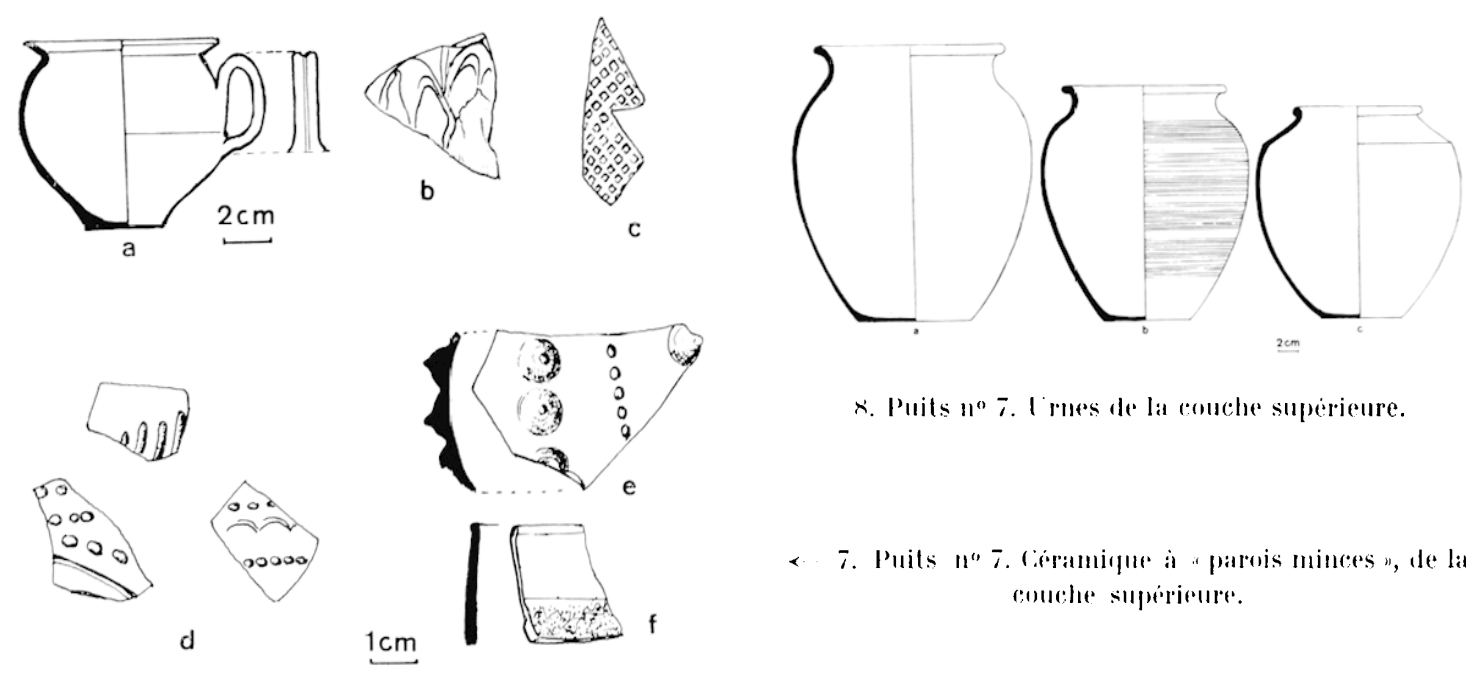

$\rtimes$ Puits $n^{\circ} 7.1$ mes de la couche superienre.

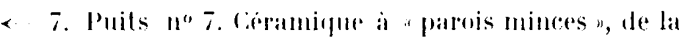
enuche superionte.

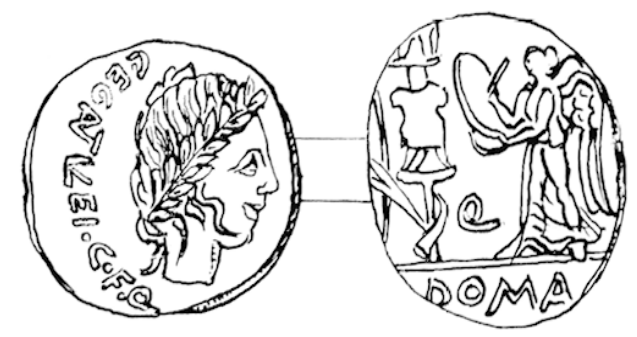

a

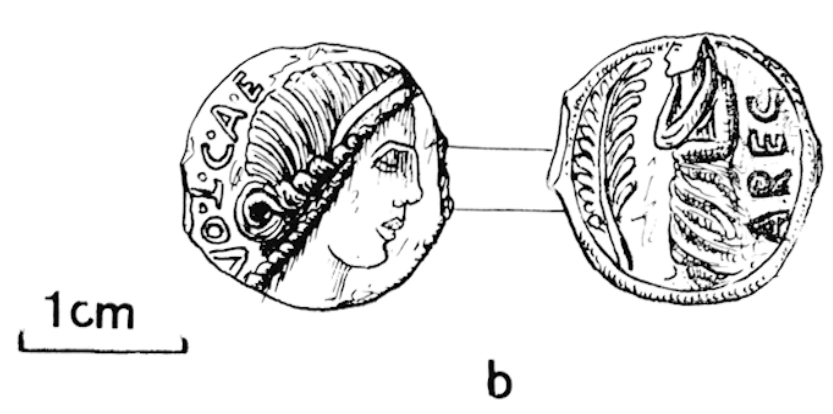

9. Pieces de monnaie du puits n" 7 : quinaire d'lignatuleius et petit bronze des Voldues Arécomiques.

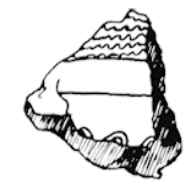

a

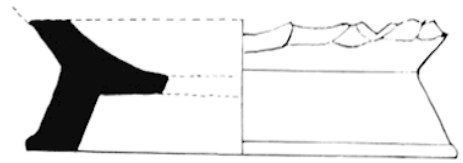

b

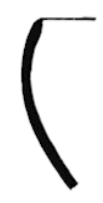

c

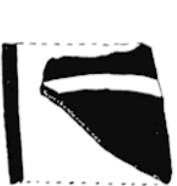

$\mathrm{g}$

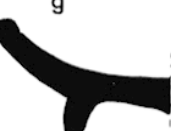

$1 \mathrm{~cm}$

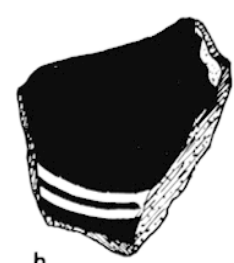

$\mathrm{h}$

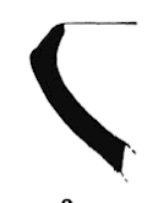

e

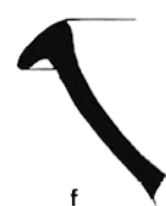

f

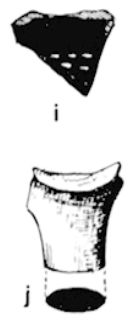

10. Puits $n^{\circ} 7$. Poterie grise imitation phocenenne el campaniemm.
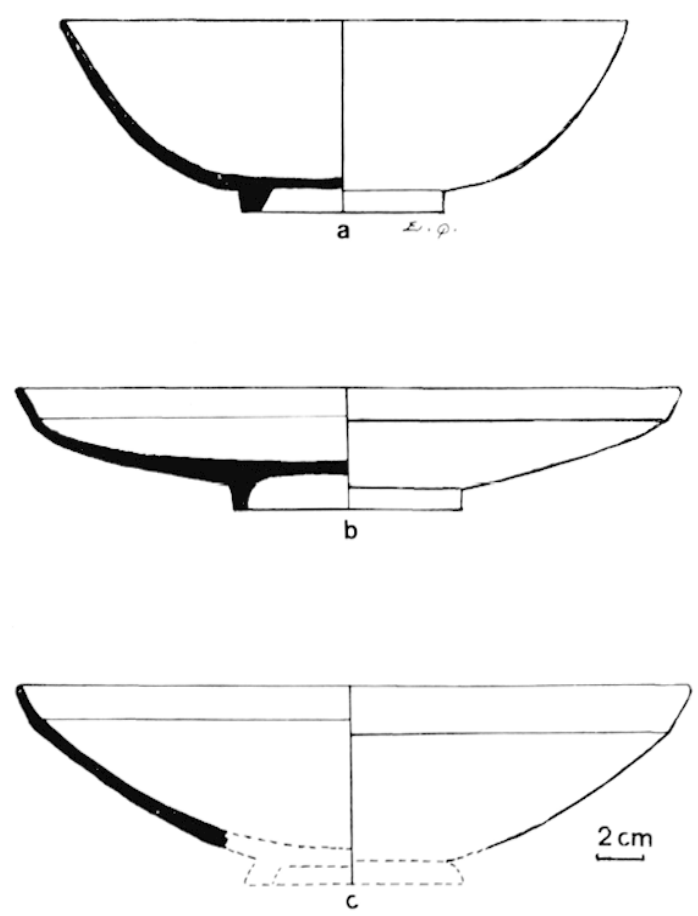

11. Puits $n^{\circ} 7$. Formes de vases, imitation regionale du campanien. 
Revers: RON.I. La victoire, écrivant, sur le bouclier d'un trophée dont le casque est orné de cornes de taureau; au pied du trophée. un carnyx ou trompelte militaire gauloise. Dans le champ, la lellre Q(uinarius). Poids ; 1 gr. 6(6) (fig. 9 a).

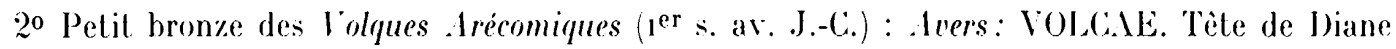
diadémée, à droite, avec, devant, une couronne de lauriers. ... Revers: . IREC-Personnage deboul, revêtu d'une togre, ì gauche, avec palme ${ }^{17}$ (fig. 9 b).

\section{J.- Polerie fine d'importalion}

1. Imilation de polerie phoréenne. Quatre tessons, en argile grise semi-dure, avec traces d'engobe plus foncé : deux fragments épais de cols de vases dont l'un est orné d'une zone de traits finement sinueux horizontaux, séparés par une bande unie d'une rangée de trails ondés (fig. 10a), et deux pieds incomplets, profonds et obliques, de grandes coupes (fig. $10 \mathrm{~b}$ ).

B. Irolocampanien: nous classons dans cette catégorie 3 petils fragments de bord el panse de coupelles a vernis noir tries brillant, et quelques menus morceaux, dont un avec trou de réparation (fig. 10 ) cd).

(. Campanien $.^{18}: 2$ fragments de coupes caractéristiques de cette céramique. l'une a bord rentrant anqulaire, l'autre à rebord pendant ${ }^{19}$ (fig. 10 e, f).

I). Campanien B: fragment de pied de coupe avec double bande circulaire peinte en blanc a l'intérieur, de paroi avec une seule bande peinte, de fond a guillochis intérieur. d'anse étroite (figr. $10 \%$ h i j).

E. "Campanien", imilation régionale, probablement massaliole: c'est la céramique" fine la plus abondamment représentée. L'argile qui la constitue est rougeàtre, pàle, homogene al dure, recouverte par un vernis soit noir mat ou peu brillant, soit marron foncé de nuances diverses à éclat métallique, pouvant ètre de teintes différentes sur le mềne vase.

Trois types de récipients. Le premier est une écuelle en forme de saladier, sorte de (alotte sphérique s'élevant d'un pied reux (fig. 11 a) ${ }^{20}$. Sur le fond intérieur, on voit quelquefois un ou deux sillons circulaires concentriques, ct aussi, souvent, la trace du pied d'un autre récipient semblable, qui s'est imprimé lors de l'empilement des vases lans le

16. F. Bassion, Mescriplion des monnaies de la Rejublipne Romaine, I, p. 475 et 176.

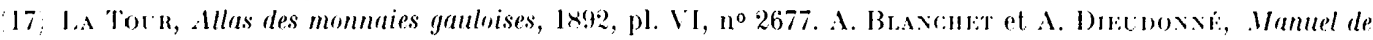
Numismalique francaise, 1912, I, p. 13 fig. 56. l.es volques Arécomiques ataient localisés dans lacluel département

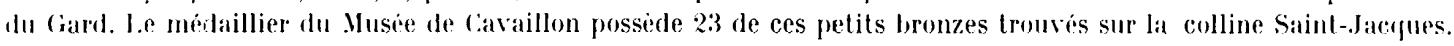
Il n'est pas sans intribl de noter la presence d'un quinare d'Egnaluleins, associc à 2 p. b. des loblues et d'autres pioces de Massalia, dans une habitation hellénistique de Glamum du $\mathrm{I}^{\mathrm{r}}$ siecle ax. J.-(:. : H. Ros.taxb, Fonilles de Gilanum

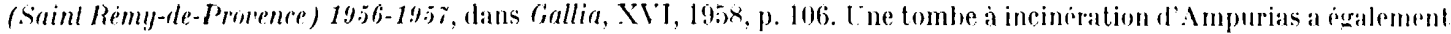

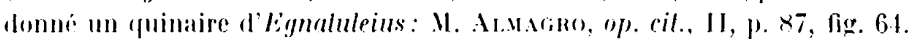

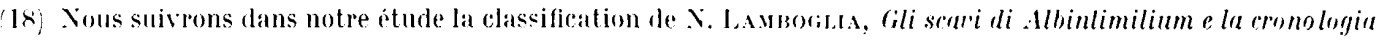
della ceramia remana, Istilulo internazionale di studi liguri, Bordighera, 1950, p. 65 et suivantes.

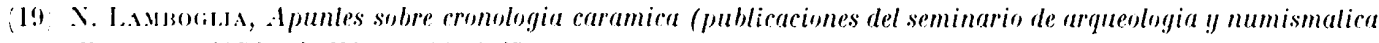
aragmese ), Zaragozal, 19\%2, pl. 11, no; 34 et 35.

(20), Cette forme de vase ne se retrouve exactement ni dans la Campanienne $A$, ni dans la $B$ ni mome dans la $C$.

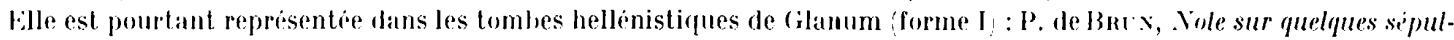
lures gallo-grecques des environs de saint-Rém!-de-Procence (B.-r.-R.), E.xtr. des Mém. de la soc. d'hist. el d'arch. de

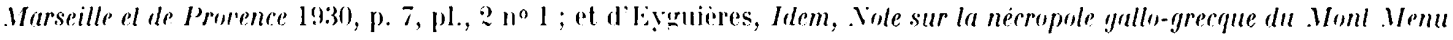
près d E!ygniores (B. du R.), extr. des . Vem. de l'inslil. hisl. de Pronence, 1931, p. 1 el 13. 
PLITS ET FOSSES IDE G.AVAILLON

9
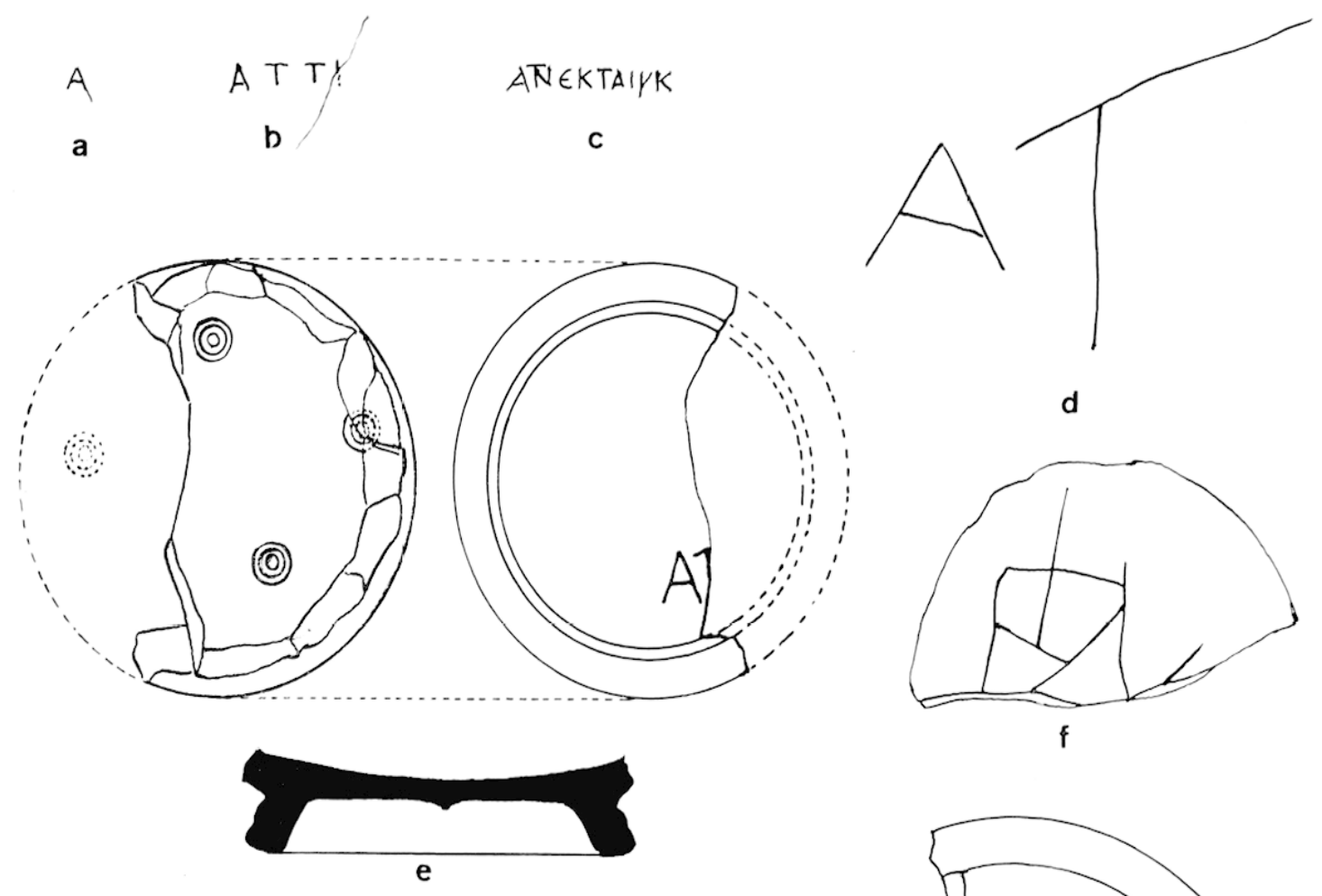

d
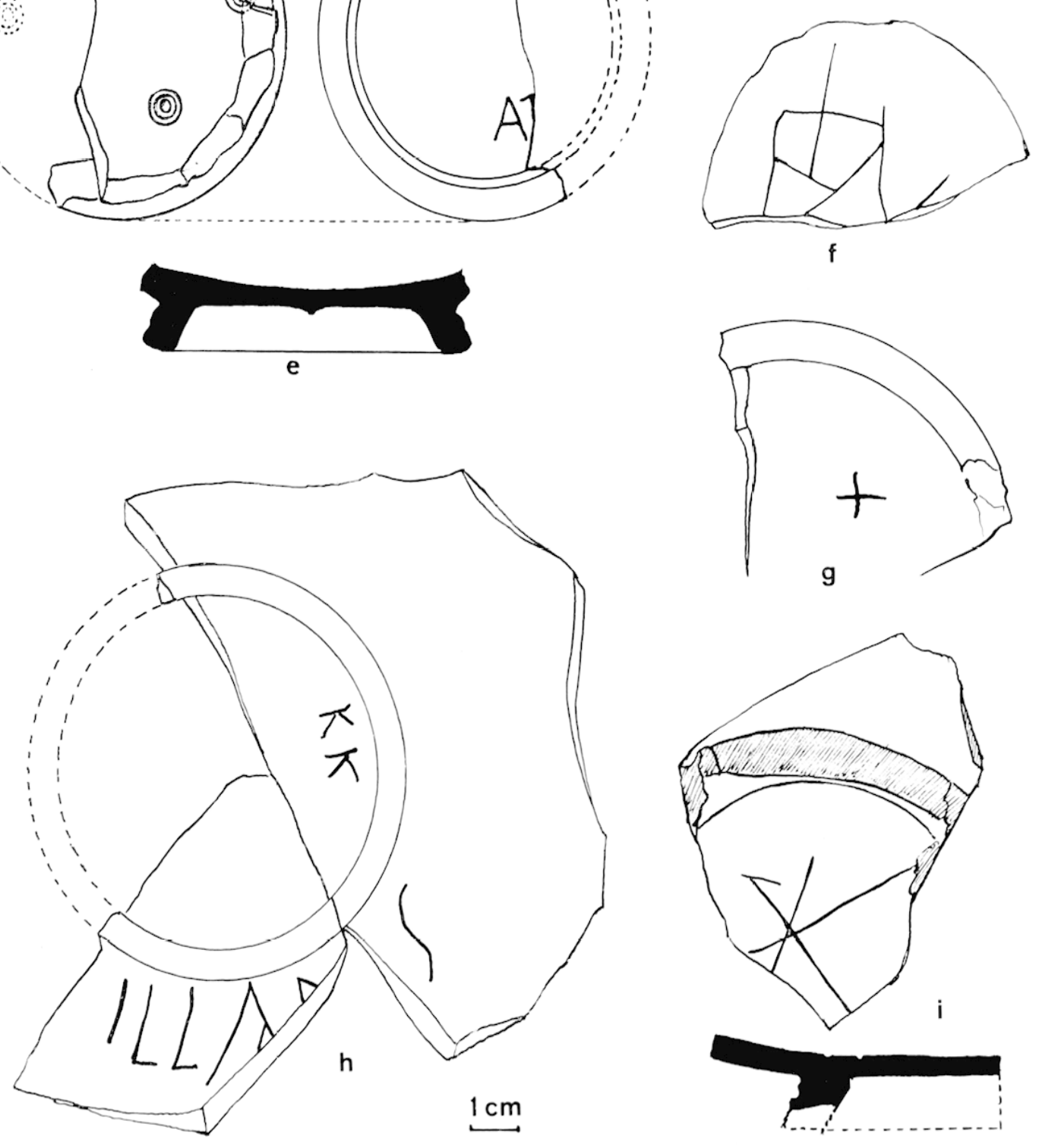

12. Puits no 7 . Graffiti sur poterie imitation (ampanien. 
four du potier. 4 ont été reconstituécs. L'une mesure $8 \mathrm{~cm}$. de hauteur et $24 \mathrm{~cm}$. de diametre; une autre $(H: 7,2 \mathrm{~cm} .-$ I). $0 .: 24,2 \mathrm{~cm}$.) a la lettre A gravée sous le pied (ligr. 12 a)

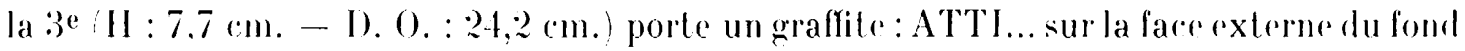
(fig. 1.2 b) ; la $4^{\mathrm{e}}$ a une hauteur de $7,4 \mathrm{~cm}$. et un diametre de $24.17 \mathrm{~cm}$. sont incompletes.

bes graffites se remarquent sur 6 d'entre elles, soit sur la paroi externe (fig. 12 d at fig. 13 a), soit sur la partic extérieure du fond (fig. 12 c f g i ct fig. 13 b), soit sur l'un ot l'aulre a la fois (fig. 12 h). Ont été recueillis encore: 120 fragments de bords, 30 de fond ot 143 de panses, d'une vingtaine de récipients de mème espèce. La variété à vernis noir comprend 2 écuelles incomplètes et 73 fragments de bord, 15 de fonds, 79 de parois, d'une dizaine d'autres vases de forme semblable.

Le druxième type de récipient, moins abondamment représenté, est une patère basse a pied creux profond droit et bord oblique (fig. 11 b) ${ }^{21} .6$ exemplaires incomplets : 1 à vernis marron brillant, 1 à vernis noir mat et pied marron, 2 à vernis noir à reflets métalliques par plares, 2 en argile pâle à vernis marron clair et une cinquantaine de fragments de 5) autres à vernis noir plus ou moins brillant.

Le troisième type (un seul exemplaire incomplet) est une patère haute, forme intermédiaire entre le 1 er et le 2 e, à parois légèrement courbes et bord oblique, court, faisant un angle très obtus avec la panse (argile marron clair à vernis foncé très écaillé) (fig. 11 c).

Signalons encore, dans la catégorie des écuelles, un fragment en argile très pâle à vernis marron sans éclat métallique, un pied incomplet aver intérieurement 3 petits cercles concentriques en 4 endroits et un graffite extérieur: A T. (fig. 12 e) et 3 fragments en argile jaune à enduit histre clair ; dans la forme patère, quelques tessons en argile jaunâtre à enduit brun clair, qui sont des imitations du Campanien tardif.

F. Campanien $C: 1^{0}$ (irand plateau rirculaire, en terre cuite gris cendré a vernis gris foncé brillant s'écaillant facilement et ne recourrant pas la totalité extérieure du récipient lle pied et une zone avoisinante en sont exempts), bord légèrement évasé, pied bas creux oblique, à rainure. 1 l'intérieur, autour d'un petit cercle central sont disposés concentriquement 2 sillons et une bande hachurée (fig. $14 \mathrm{c})\left(\mathrm{H} .: 5.5 \mathrm{~cm} . \quad\right.$ D. $0 .: 39 \mathrm{~cm} .-$ D. base $: 14,3 \mathrm{~cm} .{ }^{22} .132$ fragments de grands plateaux du mème type dont un pied incomplet avec graflite répété (fig. 13 e), représentent 4 autres récipients semblables.

20 Patère, réduction du grand plateau précédent. I l'intérieur, zone circulaire hachurée limitée par 2 sillons concentriques autour d'un petil cercle central $(11 .: 3.2 \mathrm{~cm} . \quad$ D. $(0 .: 21 \mathrm{~cm} .-$ D). B.: $8 \mathrm{~cm}, 7$ ). Deux exemplpires idenliques (fig. $14 \mathrm{~d}$.)

$3^{0}$ Bol a pied large, panse obtuse, évasée vers le haut, levre légierement abaltue ${ }^{23}$; sous le pied graffite disposé en cercle (fig. 13 c et 14 a). (H. : $4 \mathrm{~cm}, 1$ - I). (0. : $14 \mathrm{~cm}, 8-\mathrm{D}$ ). B. : $7 \mathrm{~cm}$ ).

$4^{\circ} \mathrm{Bol}$ à pied très large, à orifice bordé par 2 sillons parallèles extérieurs ${ }^{24}(\mathrm{H} .: 3 \mathrm{~cm}, 8 . \mathrm{I}) .0$. : $14,8 \mathrm{~cm}$. I). B. : $8.6 \mathrm{~cm}$.) (fig. $14 \mathrm{e})$, et I bord à un seul sillon sous la lívre.

21 Cette forme est conmue dans la Campanienne B (cf. L. LAMborids, Apunles sobre cronologia Ceramica, op. cil., pl. $1 \mathrm{n}^{\circ} 7$;

(2) Ibid., A rapprocher du no 7 , pl. II.

2:3) Ibid., pl. II, no 19.

(2) Ibid.. pl. II, no 1 . 

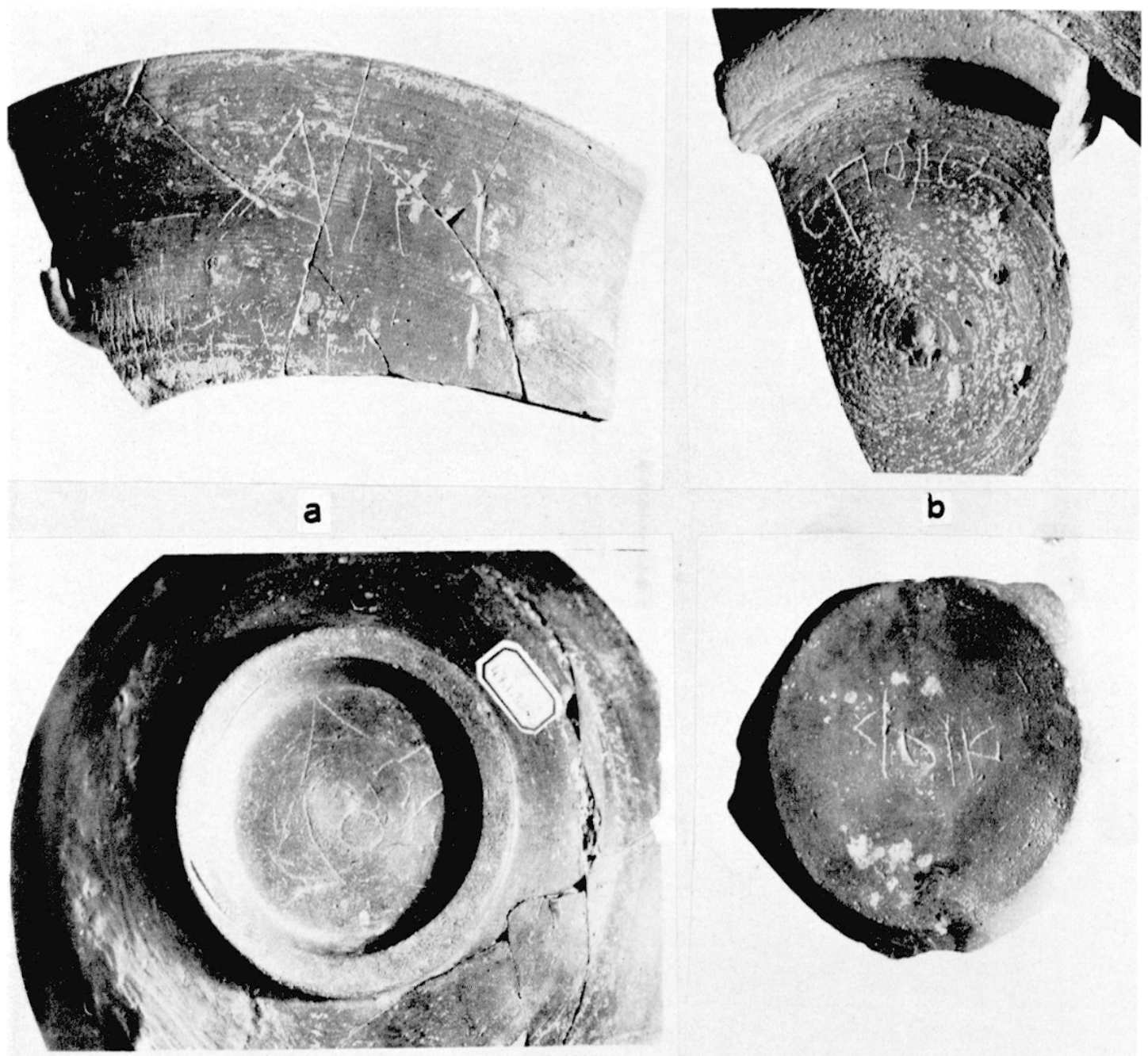

b
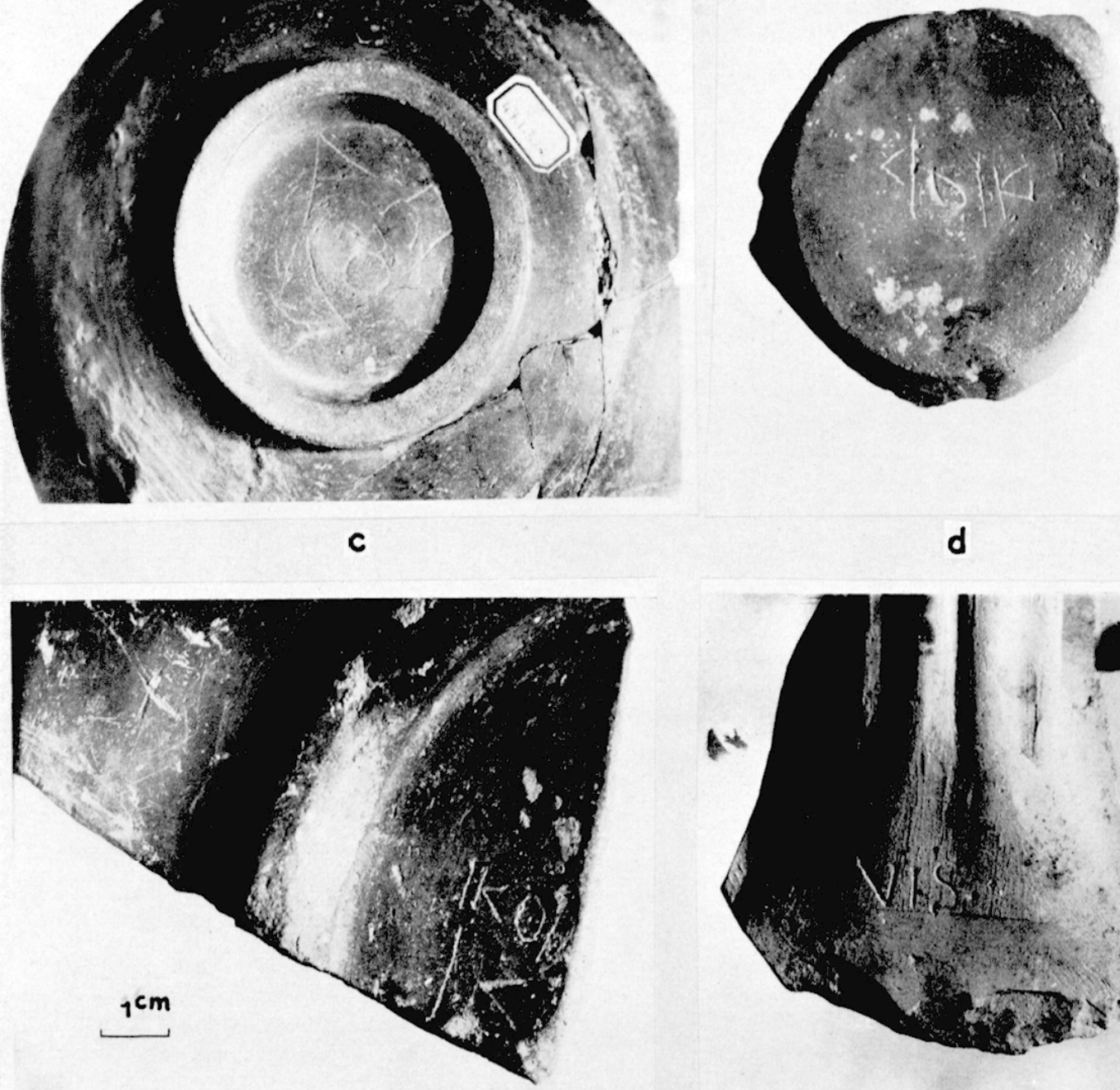

d

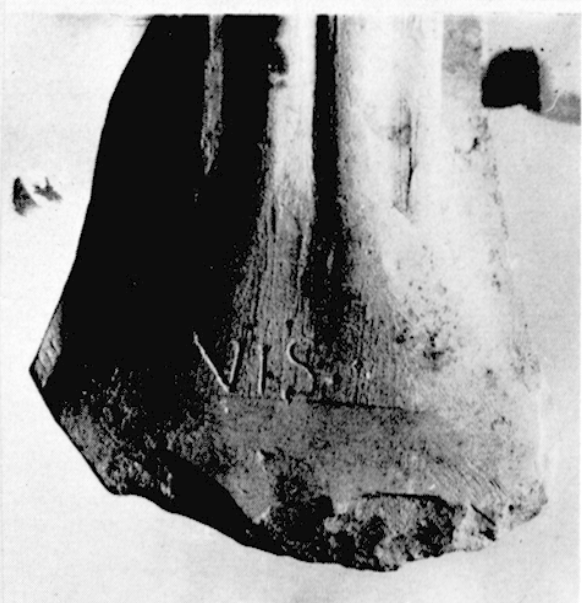

e

f

13. P'uits $n^{\circ} \overline{7}$. Riraffiti. 
5o Bol à rebord déversé aver rainure circulaire sur le dessus ${ }^{25}$ (figr. 14 b) el 23 fragments de 3 autres bols de ce type. Fignalons encore 20 fragments de bols divers de forme non précisable.

(i. Imitalion de Campanienne $\left(^{26}: 1^{0}\right.$ Petit bol en terre gris cendré dure, a engobe plus foncé, évasé a parois obliques el live arrondie (11. : 3,5$)(\mathrm{m}$. I). ().: $10.4 \mathrm{~cm} .1$ ). 13.: $4.8 \mathrm{~cm}$. ! (fic. li) a) ;

$2^{\circ}$ Petit bol a rebord épais et panse légerement galbée (fig. lis b) ;

30 Petit bol en terre grise dure à enduit foncé tris écaillé, parois obliques el bord redressi: pied en bourrelet circulaire (H. : 3,3 cm. D. (). : $9.2 \mathrm{~cm}$ (' D. P. : 4.7 ('m.) (fig. 1:) c).

$4^{0}$ Ecuelle en terre grise, panse oblique devée légerement coudée vers le bas. (1I. : 6,8 cm. 1). (). : $20 \mathrm{~cm}$. I). P. : $8,4 \mathrm{~cm}$.) (fig. 15) d);

$5^{0}$ Vase en forme de grand bol, a parois hautes, galbées. terminées par un pied bas, légerement rreux. live épaissie en bourrelel (II. : 13 ('m. I). (). : 21.5) (m. I). $13 .: 8.7$ (m. (fig. 15) e) ;

6o Deux bols incomplets a double sillon sous le bord. en argile grise a engobe gris marron:

$7^{\circ}$ Lrne globuleuse a col court coneave, el panse a cannelures horizonlales dans sa partie supérieure (le bas manque) (lig. 1:) f ${ }^{27}$;

80 foulot d'un vase en terre cuite gris clair a courerte gris foncé mate avec départ d'anse géminée au ras du bord. Ce goulol est rurieusement cloisonne horizontalement a sa base par une mince plaque d'arquile bombéc. perforée de petits trous comme un bouquelier ou rerlains pols à infusion (fig. 15) $\underline{\text { g) }}$.

II. Polerie a enduil romge inlerieur. 10 Fragment d'un grand plateau en argile arínacere de lexture granuleuse. a enduit lisse épais couleur rouge "sang de berul" peu adhérent. courant seulement la surface intérieure du vase el s'arreit ant a la part ie externe du bord fond a sillons concentriques intermes. parois un peu incurveses el rebord plat fige 16 a ${ }^{28}$. Iutre fragment de bord cpais (fig. 16 a).

20 Jalle a cole oblique el rebord lombanl. en argile claire dure compacte a enduil rouge fonces

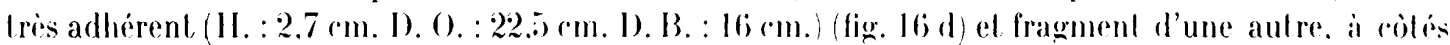
ef bords arrondis (fig. 16 b, reconstitution?.

$3^{\circ}$. Jalte incomplibe en terre cuite dure, fine, homogine el sonore, prefiguration de cerlaines: formes de la "sigillée claire" (fig. 16 re reconstilution)"

I. I'ré-sigillee. Coupelle a fond conique en dessous fragments de bord el de pied (fig. If e).

2.) Ibid., pl. II, n" 17, Ix.

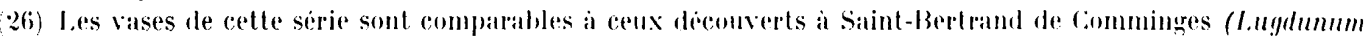

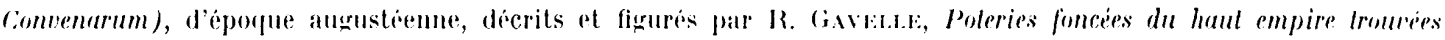

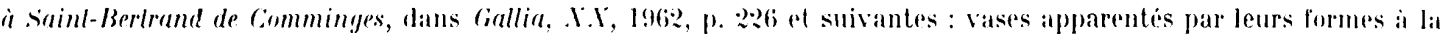
crimamique campanienne.

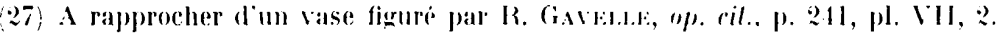

2x, l ne srande jatte semblable, qui a pu être reconstitue en entier, a ti trouve a cilanmm dans un milieu

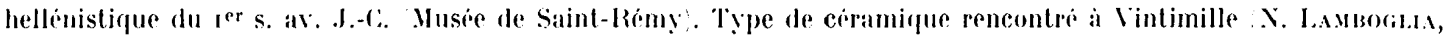

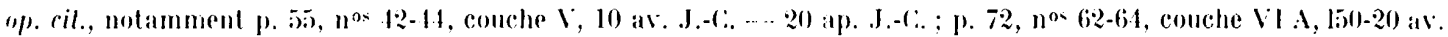

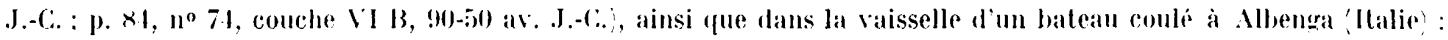
Idem, I.a nure momana di Alhenga, dans Rerue d'eludes ligures, .VIII, 1952, p. 169 et 170. forme 9 ).

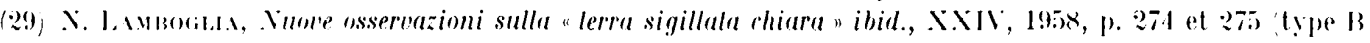



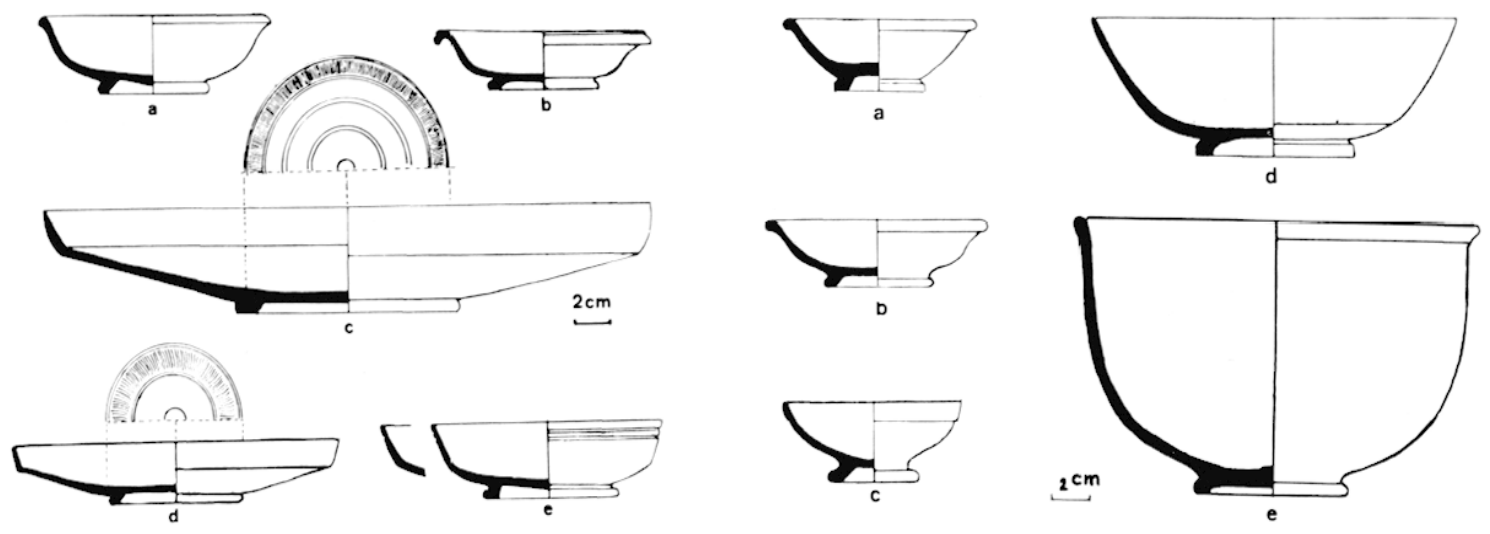

1.4. Puits no 7 . Vases en campanien l:
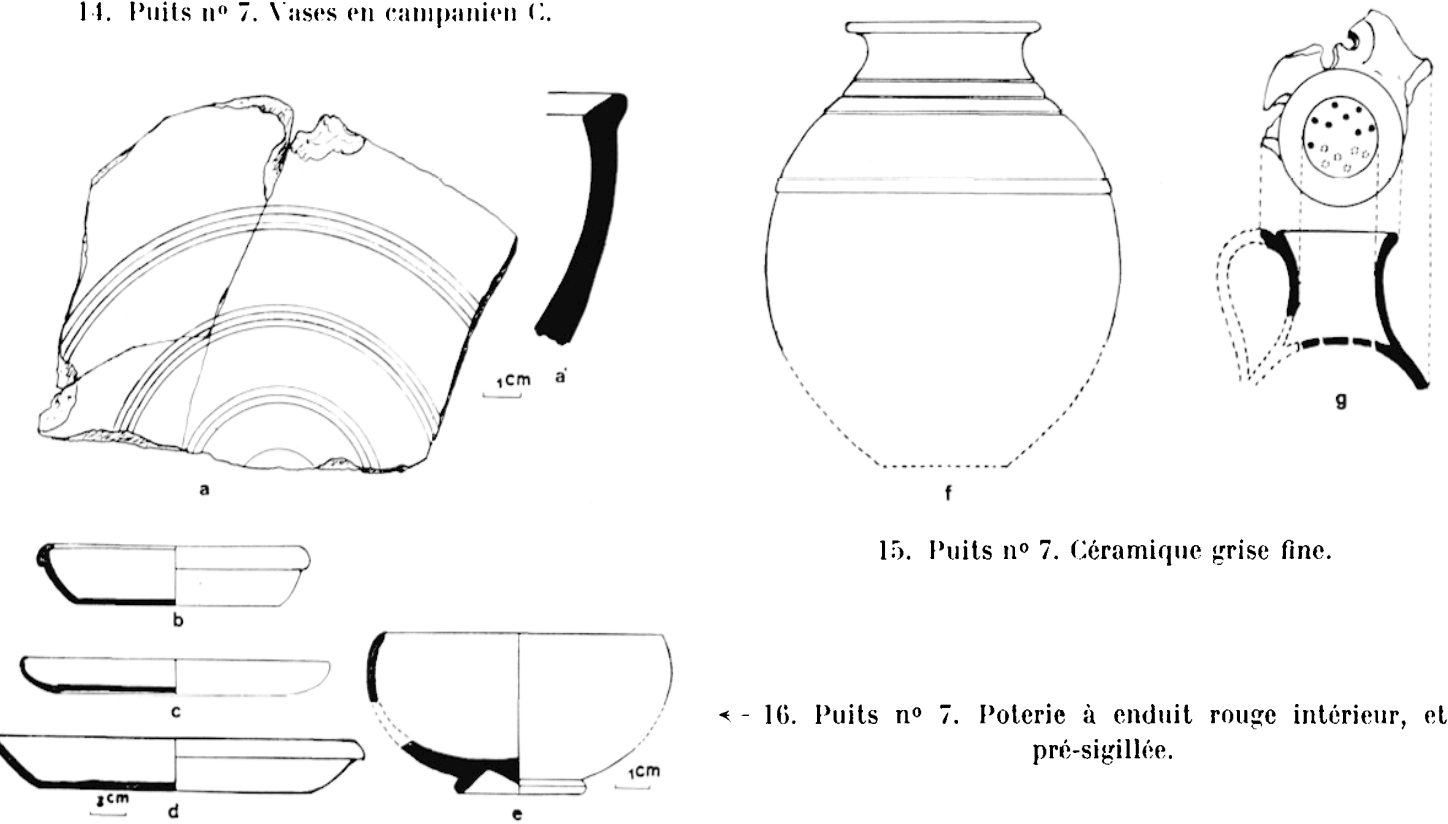

15. Puits no 7 . Céramique grise fine.

\&-16. Puits $n^{0} 7$. Poterie à enduit rouge intérieur, et pré-sigillée.

J. Terra-sigillata. a) Poterie italique unie :

1. Assietle du potier P. Hertorius (atelier de Fonte-Pozzolo près .Arezzo, Italie) ${ }^{30}$ - marque P. HER(H et E liés) répétée 4 fois sur le fond, à l'intérieur d'un cercle limité par une zone hachurée circulaire. (H. : 3,5 cm. D. 0.: $17 \mathrm{~cm}$. D. B. : 7,8 cm.) (fig. 17 b et $20 \mathrm{a}$ ).

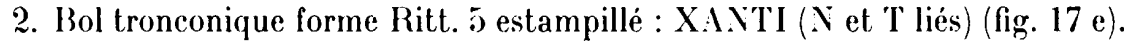

3. Fragments de fond marqué (I) VLI (fig. 17 a), de bol tronconique avec la lettre I. (fig. 17 f).

4. Bol forme Irag. 31 (variante) dont le pied manque (fig. $17 \mathrm{~g}$ ).

5. 13 fragments de bords de bols (f. Haltern 7 (fig. 17 c), d'assiettes (f. I)rag. 17 b) (fig. 17 d) et de pieds avec zone circulaire hachurée intérieure (fig. 17 h).

(30) Som de potier connu a Glanum : II. Rol.taxn, Inscriplions antiques de Glanum à Saint-Rémy-de-Propence, dans Gallia, 11,1944, p. $207, n^{\circ} 32$; a liréjus fond de récipient estampillé : PHERT, avec ligature PHE, dans les couches hasses de la Butte, Saint-Antoine, sille VII! : P. A. Fivinak, Fonilles à la ciladelle meridionale de Forum Julii (Frijus, Var) en 1955 dans Gallia, XIV, 1956, p. 44 et 16, n. 17, datée par l'auteur du dernier quart du ier siècle avant notre ire; à Ensérune (fraqt. de coupelle marquée PHElrT (H et F liés) dans la couche supérieure de la derniere phase de l'habitat $(-225-30): \mathrm{J}$. JAxNoray, Ensérune. Comlribulion à l'étude des civilisalions préromaines de la gaule méridionale, 1955, p. 45.4, en note. 

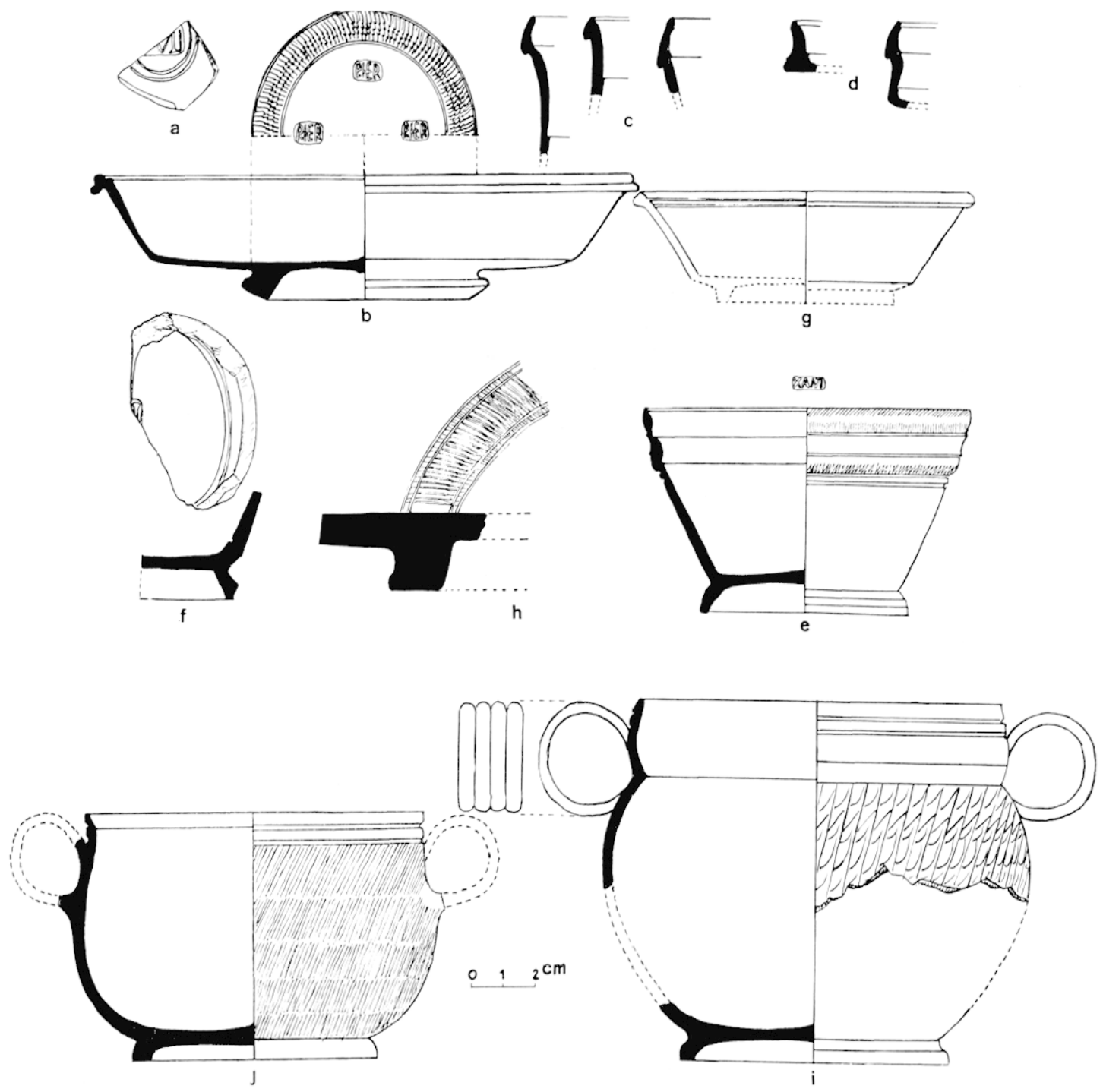

17. Puits n" T. Polerie ilatiqume.

b) Polerie arétine a panse quillochée:

1. Tasse incomplete a 2 anses annulaires, larges. a :3 sillons verticaux, pied has peu profond. panse arrondie (fig. 17 i).

2. Tasse a pied large, borl a 2 sillons paralliles horizonl aux, anses annulaires simples (fig. $17 \mathrm{j}$.

3. Fragments de panses el pied de 4 aul res lasses similaires.

K. Polerie peinle de Lezon.r. (P. de D.). 1. Elégante poliche a panse galbée col court bord arrondi. qui a pu être reconstiluée en entier, sauf la base. (H. : 22,2 cm. I). $0 .: 7,7$ cm. I). B. : 6,7 cm.). (fig. 18 et fig. 20 (·). I'argile qui la constilue est rougealre, fine, dure i engobe marron et peinture blanche légire par-dessus. Le décor, géométrique comprend de haut en bas : une rangée de bàtonnels verticaux, une frise de postes en ramaïeu marron. une zone de lignes tremblées verticales orcupant. 

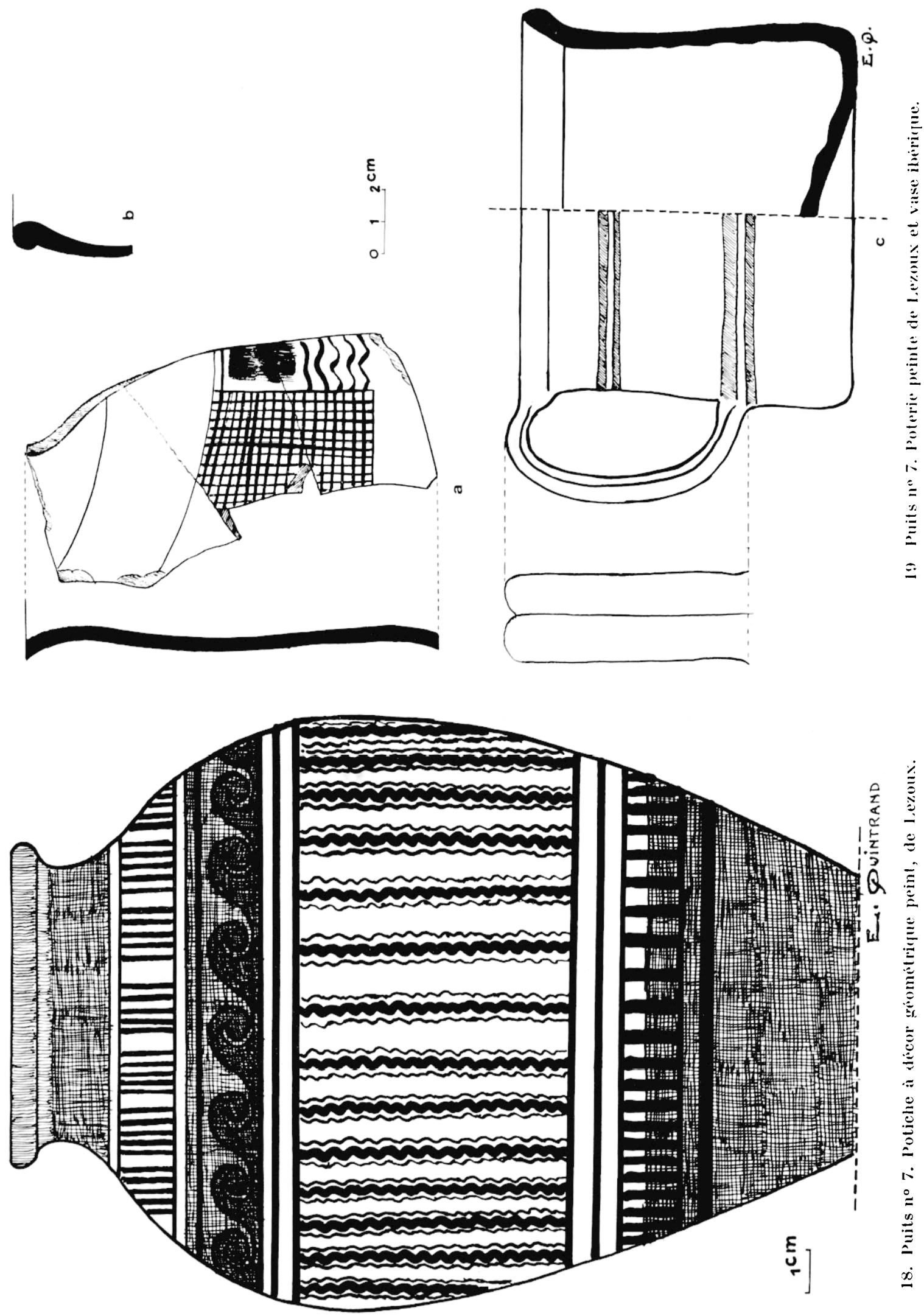
la plus grande partie de la surface de la panse. enfin une nouvelle rangée de courts batonnels vertiraux".

2. Fragments de paroi d'un grand vase orne de bandes blanches aver sinuosites horizontales: allernant avec une grille marron (fig. 19 a).

3. Bord d'une coupe is enduil blanc uni / lig. 19 b).

L. Polerie ibérique. Moilié de vase du type "pot de biere"; anse haute double, fond saillanl intérieurement, panse cylindrique ornée de 2 zones horizonl ales de bandes peint es en brun. Terre ruite jaune fine dure. légerement miracese (H. : 11.5 ('m. I). () : 12.5) ('m. I). B. 11.6 (cm.) (fig. 19) a et $2(1) d^{32}$.

M. Iases it parois minces. Celle céramique tris caractérislique 33 qui comprend des vases tous: de petite taille, en argile fine. dure. homogiene, est abondamment représentée dans le puils $n^{\circ} 7$ aver des formes variées dont nous avons pu reconstiluer la majeure partie. Nous distinguerons les récipients unis. ceux à ornements en relief barbolinés, el les vases à décor en creux.

a) Vases unis: 1. Petite urne de forme oroüde tronguée. en aryile rougeàl re panse lisse in rour-

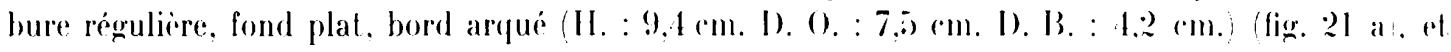
8 autres de mème lype, incomplites.

2. Petile urne dont la panse présente tout autour fi dépressions ovalaires profondes. irréguliares. (1I. : 9 cm. I). $0 .: 7.2 \mathrm{~cm}$. I). B. : 4.2 (m).), (iir. 21 b).

3. Vase élevé, a panse ovïode rél rérie à la base où elle se termine par un pied plal circulaire. bord convexe (II. : 18.5 (m. I). 0. : $8.2 \mathrm{~cm}$. I). B. : 5 (m) (fig. $21 \mathrm{l}$ ) el 2 pieds de 2 aul res vases semblables.

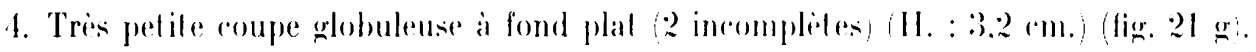

b) Tases ì ornements en relief. barbolinis:

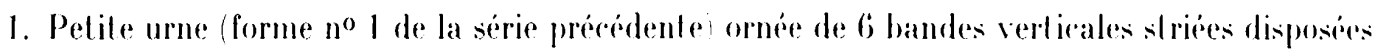

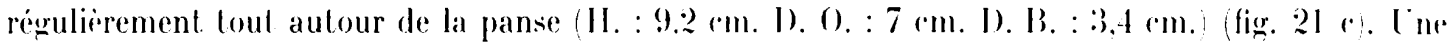
2e reconslituée et 3 autres incomplies.

2. Petile urne ovoïde à livere arrondie et pied bas. ornée sur la panse de prolubérances triangulaires allongées la pointe en bas (II. : $10.4 \mathrm{~cm}$. I). $0 .: 6,9 \mathrm{~cm}$. I). B. : $4.1 \mathrm{~cm}$.) (fig. $21 \mathrm{~h}$. I ' $\mathrm{ne}$ autre reconstituée (a pied un peu différent (fig. 21 i) et in incomplètes.

3. Vase cylindrique décoré de la mème façon (la parlie inférieure manque (lig. $21 \mathrm{j}$ ).

4. Vase allongé, rétréci ì la base, ì panse couverte de motifs virguliformes (HI. : 19,4cm. D. $0 .: 7,4 \mathrm{~cm}$. I). B. : $4.8 \mathrm{~cm}$.) (fig. $21 \mathrm{n}$. 5 bords et 60 fragments de panses.

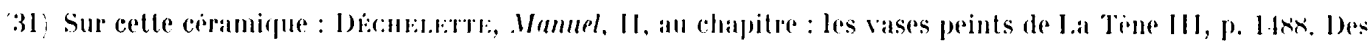
lessons de vases peints de lezoux ont ite recomms a Vintimille dans les niveaux du ler siecle at. notre ire

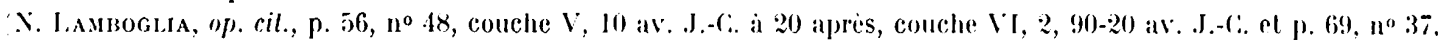
couche VI $A, 50-20$ av. J.-f.. et récemment à Vaison-ta-romaine dans un niveau augustien (foulles de l963). Cette forme de potiche élancée se retrouve dans la série des vases peints de l'oppidum de Manching, en Allemagne, qui sont

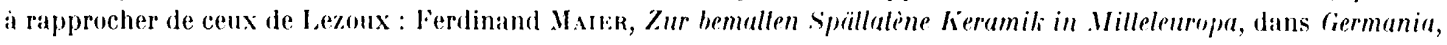
$11,1963$.

(32) Type de ceramique rencontre éralement a Vintimille dans des niveaux de la fin de l'ipoque républicaine : ‥ Lamborila, op. cil., couche VI B, p. 93.

(33) ("est celle qui constitue les vases a "pareti soltili "a parois fines, ou minces) des archeologues italiens, pien representes et datos dans les differents horizons de Vintimille decrits par x. I..umbisa (op. cil). 

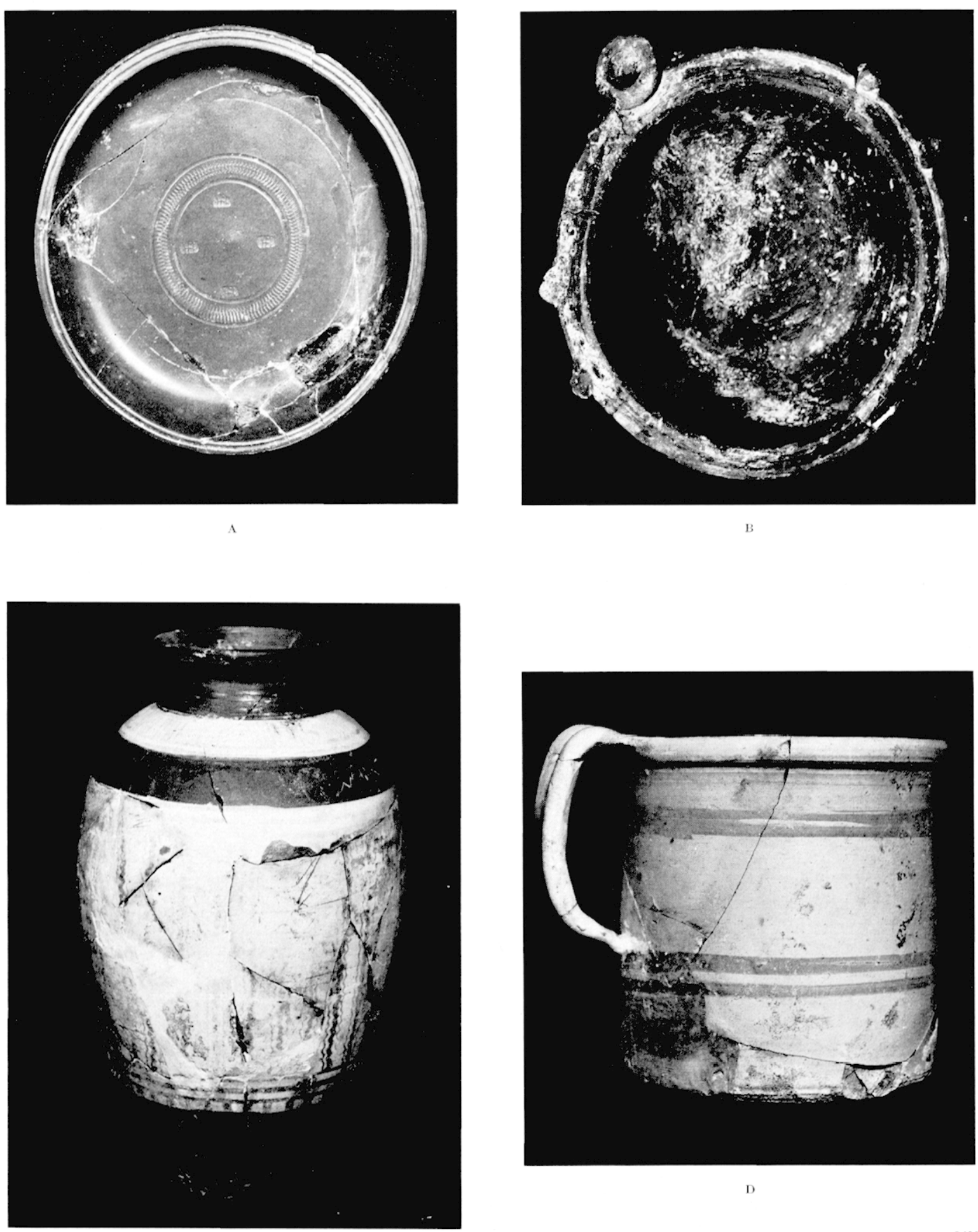

D

20. Puits $n^{\circ} 7$. Assielte du potier Herlorius, iatte a woulot, potiche de l.e\%oux et vase à anse, ilsérique. 
5. Vase allongé. en terre grise. bord droil étranglé a la base surmontant un col large, suréleve. arqué, lisse. panse à ornements semblables au type précédent (II. : 18.7 ( $\mathrm{cm}$. I). (). : 8 (cm. I). B. : 4 cm. (fig. 210$)$.

6. Ciobelet a bord droit. base plate, panse ornée sur les 2.3 inférieurs de sa hauleur de molif: claviformes obliques, (1I.: $11.2 \mathrm{~cm}$. I). 0.: $7,4 \mathrm{~cm}$. I). I3. : 4,2 (m.) el un aul re semblable. reconsliture. (fig. $21 \mathrm{f}$ ).

7. Petite urne à panse parsemée de petits triedres disposés en quinconce. verticalement. la pointe en bas. (H. : $9,4 \mathrm{~cm}$. D. $0 .: 7,6 \mathrm{~cm}$. D. $13 .: 4,8 \mathrm{~cm})$ (fig. $21 \mathrm{~d}$ ) et 3 autres incompletes.

8. Vase a pied étroit, bord en forme de petit bourrelet avec filel horizonlal au-dessous. orné romme le précédent (H. : $8,5 \mathrm{~cm}$. D. $0 .: 6,7 \mathrm{~cm}$. I). P. $3,7 \mathrm{~cm}$.) (fig. 21 e) el 1 bord d'un autre un peu plus grand.

9. Vase allongé à pied rétréci, panse à pelits triedres disposés comme les 2 récipients précédenls. (H. : 15,5 cm. D. 0.: 7 cm. I). 13. 4,1 cm.) (fig. 20) m) ef 4 incomplets.

10. Vase du potier Aro (Italie du nord).

Fragment d'un vase à parois fines. caracléristique, à décor moulé el non barbotiné représentant de petits triangles en relief. au milieu desquels se trouve un sapin stylisé (fig. 22: a) ${ }^{34}$.

11. Fragments d'un gobelet "à décor clouté " constit ué par des points en relief réunis en guirlandes (fig. 22 b). C'est la forme bien reconnaissable. décrite par F. Benoil. parmi la vaisselle importé d'origine étrusco-campanienne recueillie sur lépave de Marseille ${ }^{35}$ el rencontrée aussi i) Entremont ${ }^{36}$ el Impurias ${ }^{37}$.

c) V'ases à décor en creux: 1. Vase allongé à pied rél réci, en argile marron rlair. male. orme d'une série de lignes irrégulieres, poncluées (fig. 21 p).

2. (iobelets en terre rougeatre a panse guillochée (2 fragments de parlie inferieure) (fig. 2:2 a el d).

Mentionnons encore une soixantaine de fonds de petits récipients i parois minces dont on ne peut préciser la forme (l'un porte un nom gravé) (fig. 1:3 d) el des fragments de panses ef bords.

X. Cngnentaria. 10 Type fusiforme, a goulot étroit et pied mouluré livere abaltue, panse

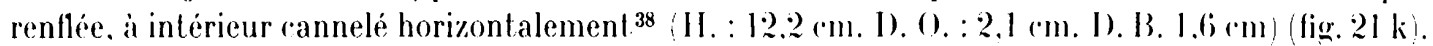

$2^{\circ}$ Type semblable, à pied plus simple. de taille plus petile el fragments de 6 aulres (fig. 21 q).

O. Poterie ì decor ocule el ponclué. ${ }^{0}$ loupe basse apode, fond saillant forlement en pointe conique a l'intérieur et bord mouluré. Paroi ornée extérieurement de 4 rangées d' " yeux " superposiess imprimés dans la paite avant cuisson ${ }^{39}$; l'argile est rougeatre tendre, bien épurée, aver engobe brun

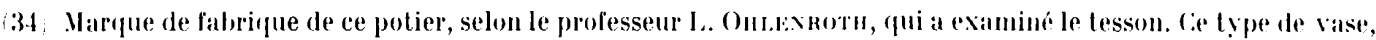

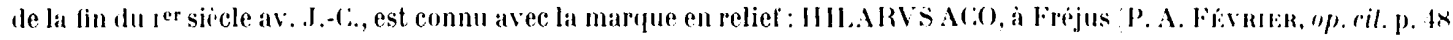

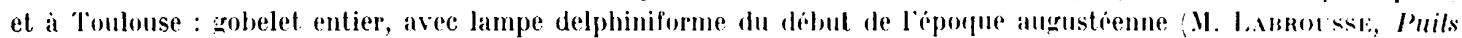
funeraires de Vieille-Toulunse, dans fiallia, XX, 196?, p. 576.

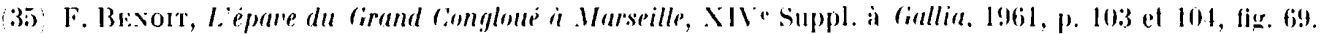

36) riallia, XIV, 1956, p. 221, fig. 6.

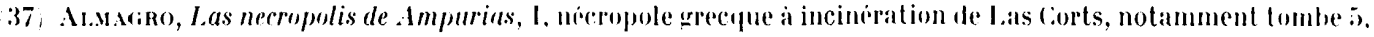

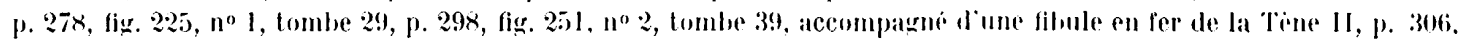

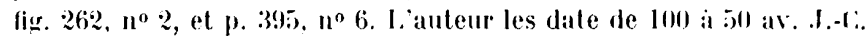

i36 Celle forme de recipient est particuliepement alondante dans les nócropoles dimpurias : M. Ar.u.riko,

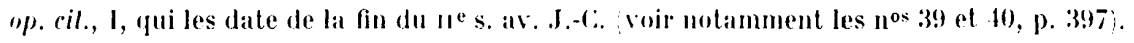

(39) Ce decor original et caractipistique, compose de "bandes horizontales de gholules en relief enchassees dans

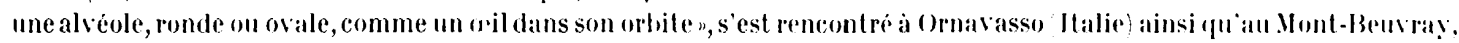

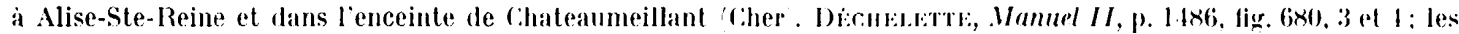

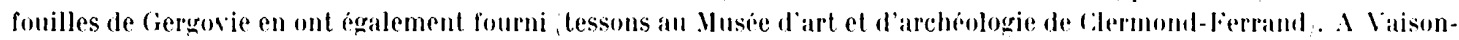

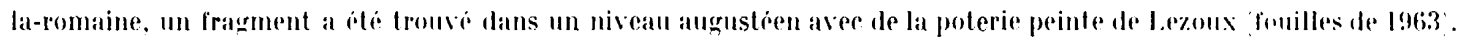



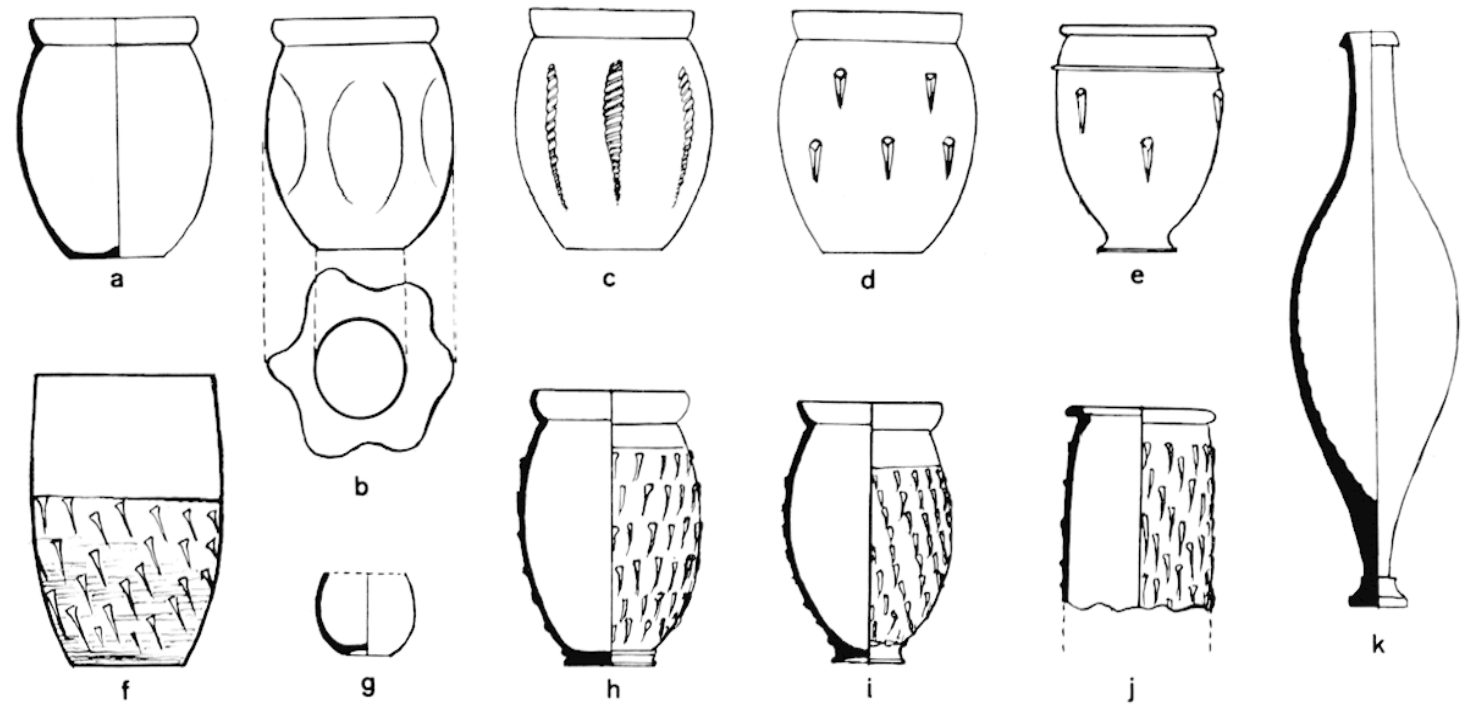

$2^{\mathrm{cm}}$
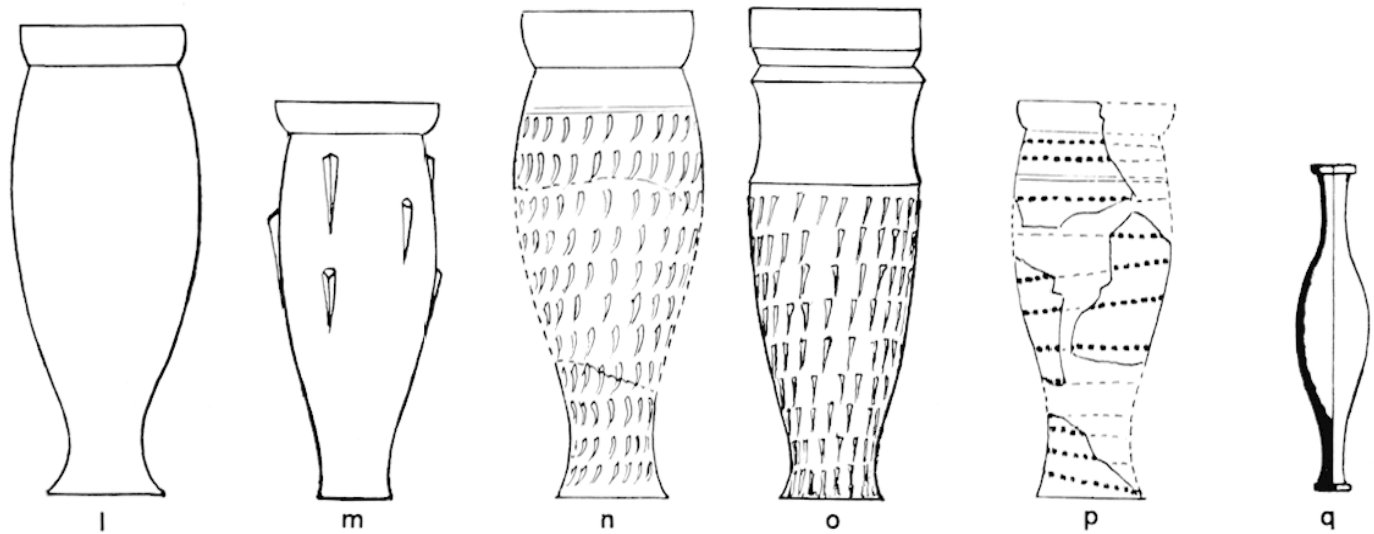

21. Puits $n^{\circ} 7$. Coramique a parois minces.

foncé a surface onclueuse (H. :5) cm. I). (). : 16,2 cm.) (fig. 23 a) - 2 fragments de deux autres coupes similaires portent. l'un 3 rangs d'« yeux » avec une bande très moulurée, l'autre 6 rangées (fig. 23, a' a"). 4 autres coupes sont représentées par 16 tessons et 1 fond conique.

$2^{\circ}$ Coupe incomplete de même forme, en argile grise tendre finement pailletée de mica. à engobe noir mat. La panse est ornée de plusieurs bandes inégales de ponctuations de forme carrée disposées horizontalement sous un bord mouluré (fig. $23 \mathrm{~b}$, reconstituée).

\section{Polerie lustrée.}

Cette céramique, probablement locale, rappelant par sa technique certaines formes du i ${ }^{\text {er }}$ Age du Fer est caractérisée par des vases non tournés dont toute la surface aussi bien externe qu'interne présente un lustrage très apparent dù au passage répété du lissoir. On peut en distinguer 2 sortes : la lustrée noire et la lustrée orangée. 

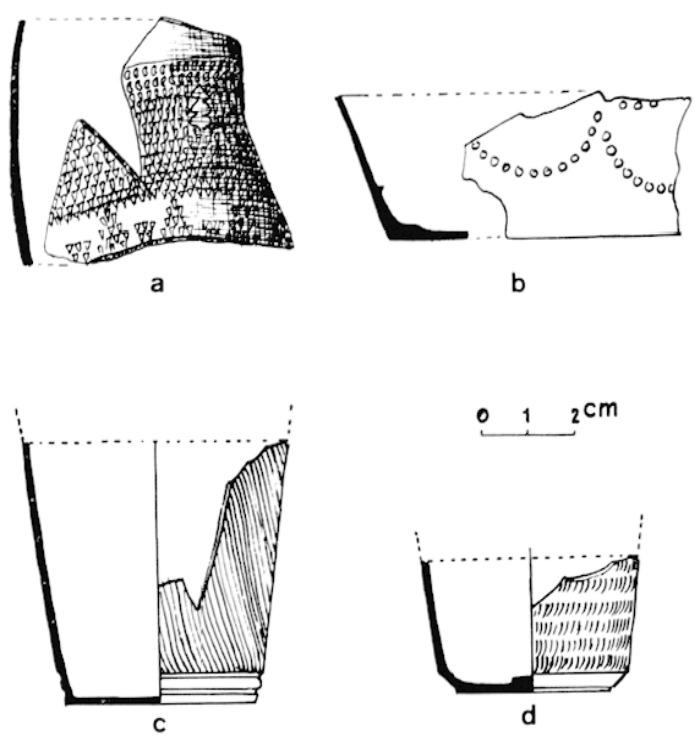

b

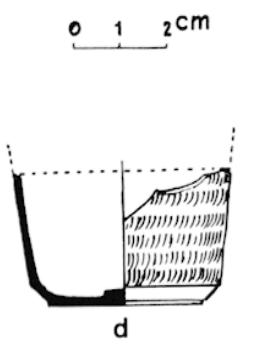

$2: 2$. Puits no 7 . Fragments de vase du potier $A\left({ }^{\circ}\right.$, de gobelet à décor "clouté " et guilloché.

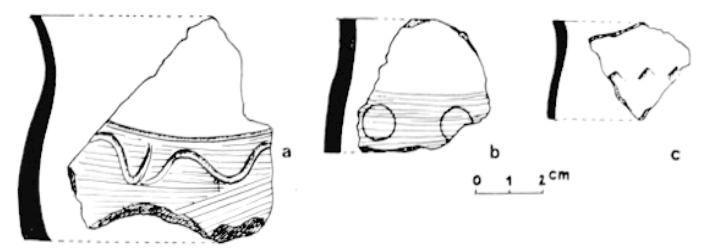

25. Puits no 7 . Decor sur panses diurnes de tradition hallstattienne.

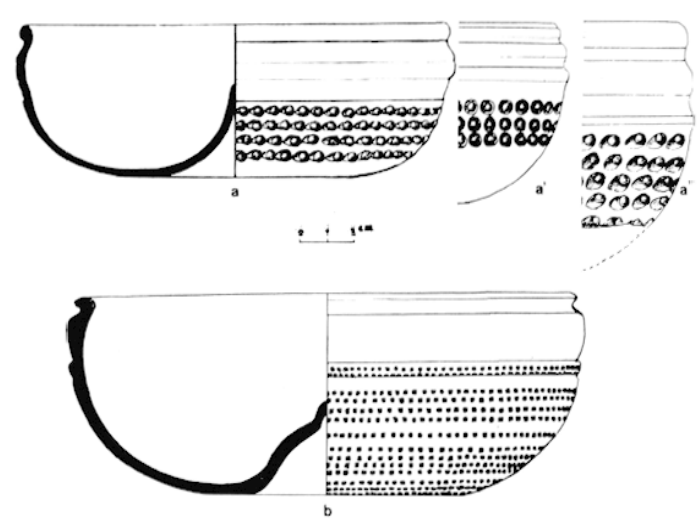

23. Puits $n^{\circ} 7$. Coupes à décor oculé et ponclui.
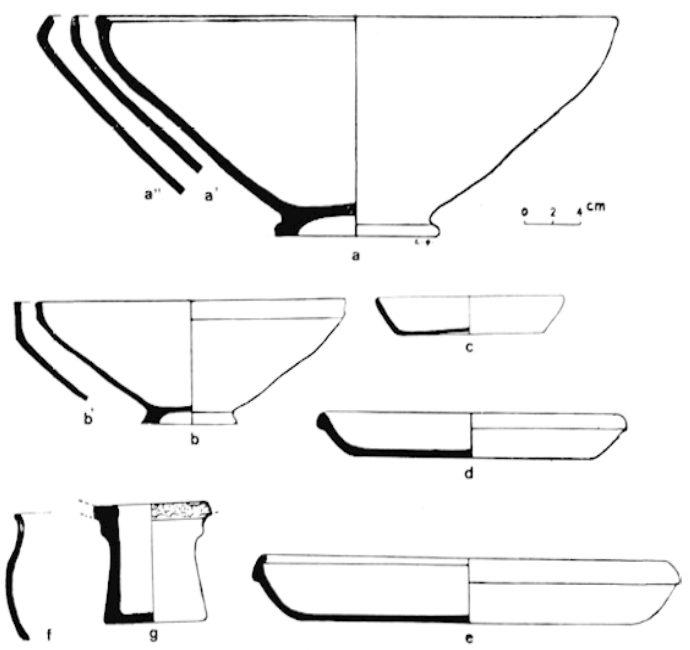

24. Puits no 7 . Vases en poteric lustrie noire et orangie.

1. Céramique lustrée noire (argile rougeàt re avec petits grains de calcaire blanc comme dégraissant, a engobe brun-noir) :

1. Grande terrine en forme de tronc de còne évasé renversé ; bord à livre un peu aplatie sur le dessus, pied creux circulaire (II. : 12,7 cm. I). 0. : $37 \mathrm{~cm}$. D. B. : $11,3 \mathrm{~cm}$.$) (fig. 24 a) et fragmenls$ d'une dizaine de récipients similaires.

2. Grande terrine de même lype à bord vertical et lìve non aplatie $(1 \mathrm{H} .: 12 \mathrm{~cm} .1) .0 .: 36,7 \mathrm{~cm}$. J). B. : $10 \mathrm{~cm}$.) (fig. $23 \mathrm{a}$ ').

3. Terrine plus petite que les 2 précédentes à bord rentrant. (H.: $8 \mathrm{~cm}$. I). (). :25) cm. I). 13. : 7,7 cm.) (fig. $24 \mathrm{a}^{\prime \prime}$ ).

4. Pied profond el haut, creux intérieurement, d'un vase de forme indéterminée (fig. $23 \mathrm{~g}$ ).

B. Céramique lustrée orangée (argile rose orangée dure mélangée de petit gravier; couleur identique à la cassure et à la surface) :

1. Terrine tronconique à pied creux oblique incisé d'une fine rainure circulaire : bord droit court (H. : 7,6 cm. D. 0. :22 cm. D. B. : $7 \mathrm{~cm}$ ) (fig. $24 \mathrm{~b}$ ) et fragments d'une aulre ì bord plus haut (fig. $24 \mathrm{~b}^{\prime}$ ). 
2. Petite assiette à fond plat et còtés obliques (II. : 2,2 cm. D. $0 .: 13,6 \mathrm{~cm}$. I). B. : $10,8 \mathrm{~cm}$.) (fig. 24 c).

3. Grande jalle a bord rentrant débordant un peu à l'extérieur (II.: $4 \mathrm{~cm}$. I). 0. : $30 \mathrm{~cm}$. D. B. : 2i) cm) (fig. $24 \mathrm{e}$ ) et fragments d'une dizaine de jattes de profil à peu près semblable.

4. Jalte plus petite i bordure teintée de brun foncé lranchant avec la couleur claire du reste du récipient (II. : : cm. 5. D. 0. : $21 \mathrm{~cm}, 5$. D. B. : $18 \mathrm{~cm}$ ) (fig. $24 \mathrm{~d}$ ) et fragments d'une $2^{\mathrm{e}}$.

5. Partie supérieure d'une urne it col incursé (fig. 24 f).

\section{Poterie commune.}

Cous désignons par ce terme toute la réramique non vernissée, de fabrique probablement locale, qui constitue les récipients d'usage domestique courant.

1. Irnes ou ollae. I part seulement trois tessons ornés d'une ligne sinueuse, d'une rangée de cercles et d'encoches triangulaires gravées (figr. 25) a b c), ayant appartenu is des urnes de Iradition hallstattienne, l'immense majorité de ces récipients, fails au tour, est du type de la Tine. Nous en distinguerons 6 formes:

Forme 1. Irne plus large que haute, en terre ruite rougeâtre ou grisìtre, dure, contenant de menus grains de dégraissant, a engobe marron clair ou foncé. Le col court el lisse se termine à l'ouverture par un rebord plat horizontal, il est orné à sa base d'une rangée d'incisions obliques, en séries de sens opposé ; la panse galbée est peignée surtout horizontalement le fond est plat, circulaire, plutiot mince. La laille de re récipient varie de 11 a

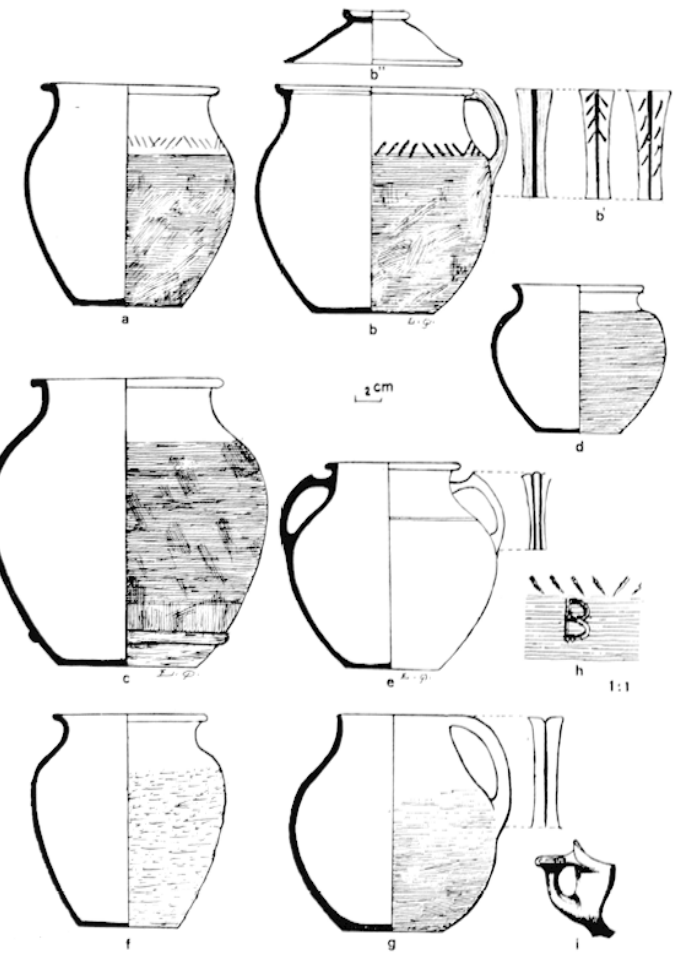

26. Puits $n^{\circ} 7$. Types d'urnes, pot et anse de lasse. 45) $\mathrm{cm}$. pour une largeur de 14 ia $48 \mathrm{~cm}$. C'est la forme de vase la plus représentée dans ce puits ${ }^{40} .21$ urnes ont été reconstituées avec presque tous leurs morreaux, 39 sont incomplètes, enfin 5000 tessons (fragments de bords de parois, de fonds) représentent a peu priss 250 autres urnes de ce type (fig. 26 a).

Forme 2. Urne de même technique, différente de la précédente :

10 par la présence d'une anse courbe prenant naissance au ras du bord du vase et aboutissant au haut de la panse; cette anse, à rainure médiane très prononcée, est tantìt ornée d'incisions obliques de même sens ou de sens contraire, ne se prolongeant pas jusqu'à sa base (fig. 26 b') ; $2^{\circ}$ par une légìre inflexion du dessus du rebord qui n'est pas rectiligne mais un peu en gouttière. Cies ollae n'atteignent pas une grande taille comme certaines des précédentes. Leur hauteur varie de $8 \mathrm{~cm}$, , a $19.2 \mathrm{~cm}$. pour une largeur de $10,3 \mathrm{~cm}$. a $21,8 \mathrm{~cm}$ (fig. $26 \mathrm{~b}$ ). 10 ont été reconstituées. 11 sont incompletes, 12 autres fragmentées. sur une panse, en haut, a été gravée avec une baguelte, avant ruisson, la lettre B (fig. 26 h).

(10) Type d'urne utilise comme olla cineraria dans les tombes gallo-grecques de Saint-Remy-de-Provence (P. de Brix, op. cil., p. 6, pl. I, 13), Lyguieres, Mouriès, Cavaillon (P. de Brtx et A. IxMoct.tx, op. cil., p. 29). 


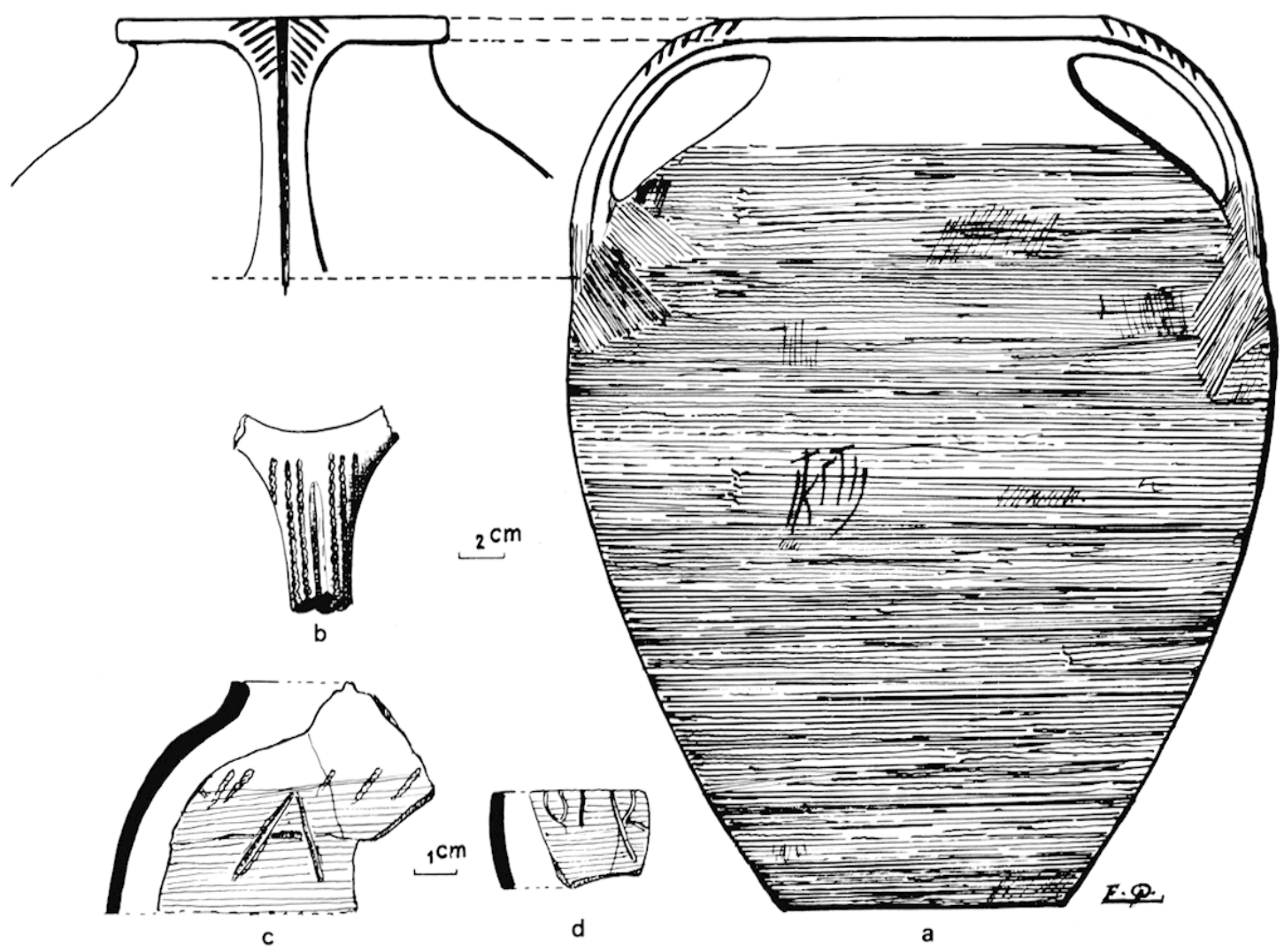

27. Puits $n^{\circ} 7$. Cirand vase a '2 anses, et grallites.

Forme 3. Line de mème technique, à engobe clair rougeàtre ou jaunailre. mais sans incisions au col. Pas d'anse. Panse a bourrelet peu saillant faisant le tour du récipient pris de la base. Reconstituées: 6 , incomplètes : 3 , et une trentaine représentées par 500 tessons (fig. 26 c).

Forme 4. Lrne de forme trapue, à livre évasée, col court, lisse, incurvé. à léger épaulemenl a la base, panse peignée régulièrement dans le sens horizontal. Lne seule a pu ćlre reconstiluée (H. : $10,5 \mathrm{~cm}$. D. (). : 10,4 cm. D. B. : $6.6 \mathrm{~cm}$ (fig. $26 \mathrm{~d}$ ). Cne trentaine de fragments représentent environ 10 urnes de ce type.

Forme 5. Lrne de forme harmonieuse, à surfare externe entièrement lisse, enduite d'un engobe marron foncé lustré, munie de 2 anses opposées a 2 rainures profondes, longitudinales, s'évasant vers le haut, coudées et aplalies a leur attachement au col. Ln sillon peu profond fait le tour du vase limitant le col et la panse ; base plate 41 (H. : $14 \mathrm{~cm}$. D. 0. : $11,2 \mathrm{~cm}$. I). 13. : $7,4 \mathrm{~cm}$.) (fig. $26 \mathrm{e})$. 2 autres reconstituées, 2 incomplètes, et 15) fragments de 4 au moins de ces récipients (il faut rattacher à cette céramique une portion de petite tasse à anse à poucier avec double sillon sur le dessus) (fig. 26 i).

Forme 6. Urne à bord évasé, col oblique lisse, et panse grossière, rugueuse extérieurement. Irgile grisâtre contenant souvent en forte quantité de petites paillettes de mica doré (3 incomplìles) (lig. $26 \mathrm{f}$ ).

11) Forme d'urne identique dans les tombes gallo-grecques d'Fyguiires (B.-d-R.), 5) ex. . P. de Вr(x, op. cil.. 1. 13 et pl. I, no 6 . 
B. Cirands vases a 2 anses. Leur forme est celle des urnes a panse peignée. mais sans incisions au col. Orifice a levres plates avec départ de 2 anses courbes, verticales. dans leur prolongement, se fixant au haut de la panse ; ces anses sont fendues par un profond sillon médian et striées en "arète de poisson" à leur sommet (fig. 27 a) où à cannelures parallèles verticales (fig. 27 b) (H. : $38.5 \mathrm{~cm}$. D. O. : $16 \mathrm{~cm}$. D. B. : $13.5 \mathrm{~cm}$.) 4 reconstituées. 2 incomplètes et 4 autres fragmentées. 3 graffiti ont été relevés sur panse de re type de rase (fig. 25) c d).

C. Combercles d'olla. Ils ont tous la mème forme conique trìs évasée, avee pied creux circulaire peu profond, en argile identique a celle des vases qu'ils recouvraient (fig. $\left.26 b^{\prime \prime}\right)$. Leur taille varie de $11 \mathrm{~cm}$. de diamètre et $2,8 \mathrm{~cm}$. de hauteur i $30.7 \mathrm{~cm}$. el $9.5 \mathrm{~cm}$. 21 ont été reconstit ués, 5) sont incomplets, une quinzaine sont représentés par 97 fragments.

I). Marmile. Récipient en terre cuite marron noiraitre mate. a panse ef fond arrondi, a parois fleves avec rebord pendant. (H. : $18 \mathrm{~cm}$. D). 0. : $30.8 \mathrm{~cm}$.) (figr. 28 a). sion couvercle a la forme de ceux qui couvrent les urnes, mais les bords sont rentrants (fig. 28 a'). Che autre marmite a été reconstituée, et 70 fragments se rapportent a 5) aulres récipients du mème grenre. avec rebord de forme variée (fir. $28 \mathrm{a}^{\prime \prime}$ ).

E. Bassine. Vaste vaisseau tronconique d parois légèrement incurvées, peignées grossièrement, munies de 2 anses horizontales pleines, en "oreille ". Le bord plat est orné sur le dessus d'incisions disposées comme sur le col des urnes, tantòt dans un sens, tantòt dans l'autre. (II. : $16 \mathrm{~cm}, 7$. D. (). : 50 cm. D. B. : $30 \mathrm{~cm}, 5)$ (fig. $28 \mathrm{c}$ ). 141 fragments de 4 autres bassines semblables. 1 signaler un tesson avec feuille de buis (Buxus

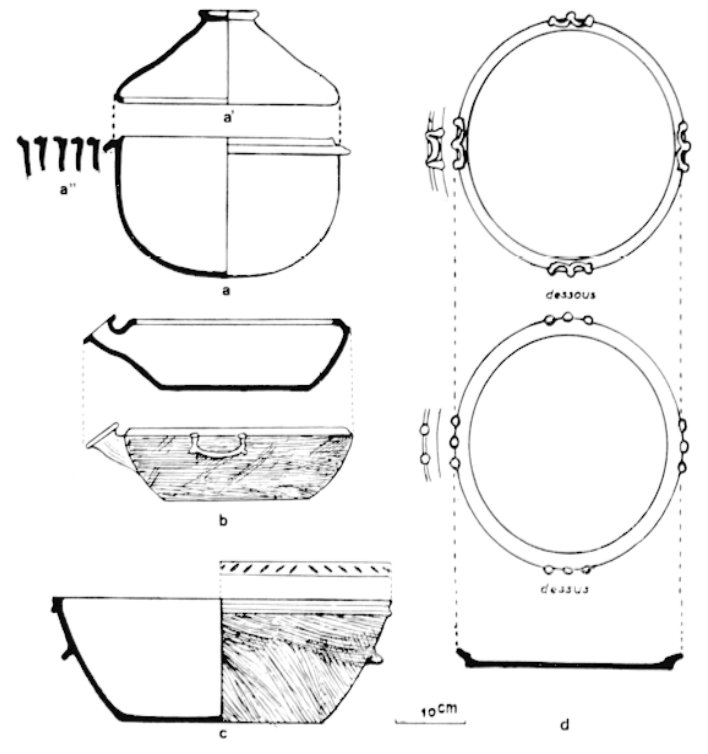

28. Puits no 7. Marmite, jatte a goulot, bassine et plateau, en poterie commune. sempervirens $L$.) conservée dans l'argile.

F. Ilatean ì pain. ('est une plaque circulaire de terre cuite marron clair, grossiire, rugueuse en dessous, lissée au-dessus, aver un très court bord oblique muni de 4 anses horizontales appliquées, simples colombins irréguliers aplatis sur le haut ou ils forment 3 boutons (H. : $2,5 \mathrm{~cm}$. D. : $40 \mathrm{~cm}$.) (lig. 28 d) et quelquefois 2. 6 autres plateaux sont représentés par 110 fragments. L'un était piqueté intérieurement de nombreux trous ne le perforant pas complètement. Un lesson contient encore une feuille de prunier épineux (prunus spinosa L.) prise dans l'argile.

(i. Jalle ì goulot. sorte de terrine basse à parois peignées, courbes, bord rentrant et fond large. plat. Ln groulot court, ì orifice oblique et lèvre retroussée, est fixé sous le bord, et 2 anses, de même lacture que celle des plateaux décrits précédemment, sont appliquées contre la paroi extérieure. rapprochées du goulot (fig. $28 \mathrm{~b}$ ). 3 ont été reconstituées : la plus grande mesure $9.8 \mathrm{~cm}$. de haut et $333 \mathrm{~cm}$. de diamirtre í l'ouverture; la plus petite $7,2 \mathrm{~cm}$. de haut et $25,8 \mathrm{~cm}$. de diamitre (fig. $20 \mathrm{~b}$ ). 7 autres sont incomplites et 194 fragments représentent une dizaine de ces récipients.

H. Pol genre pichel. Vase en argaile rouge brique, dure, sans engobe, bien tourné, à ouverture retrécie sans rebord, panse galbée, anse étroite verticale à profond sillon médian, joignant le ras du bord au bas du col ; fond plat (H. : $15 \mathrm{~cm}$. I). $0 .: 8,8 \mathrm{~cm}$. D. B. : $8 \mathrm{~cm}$.) (fig. $26 \mathrm{~g}) .4$ fragments de bord el anses appartiennent à 3 autres récipients semblables. 

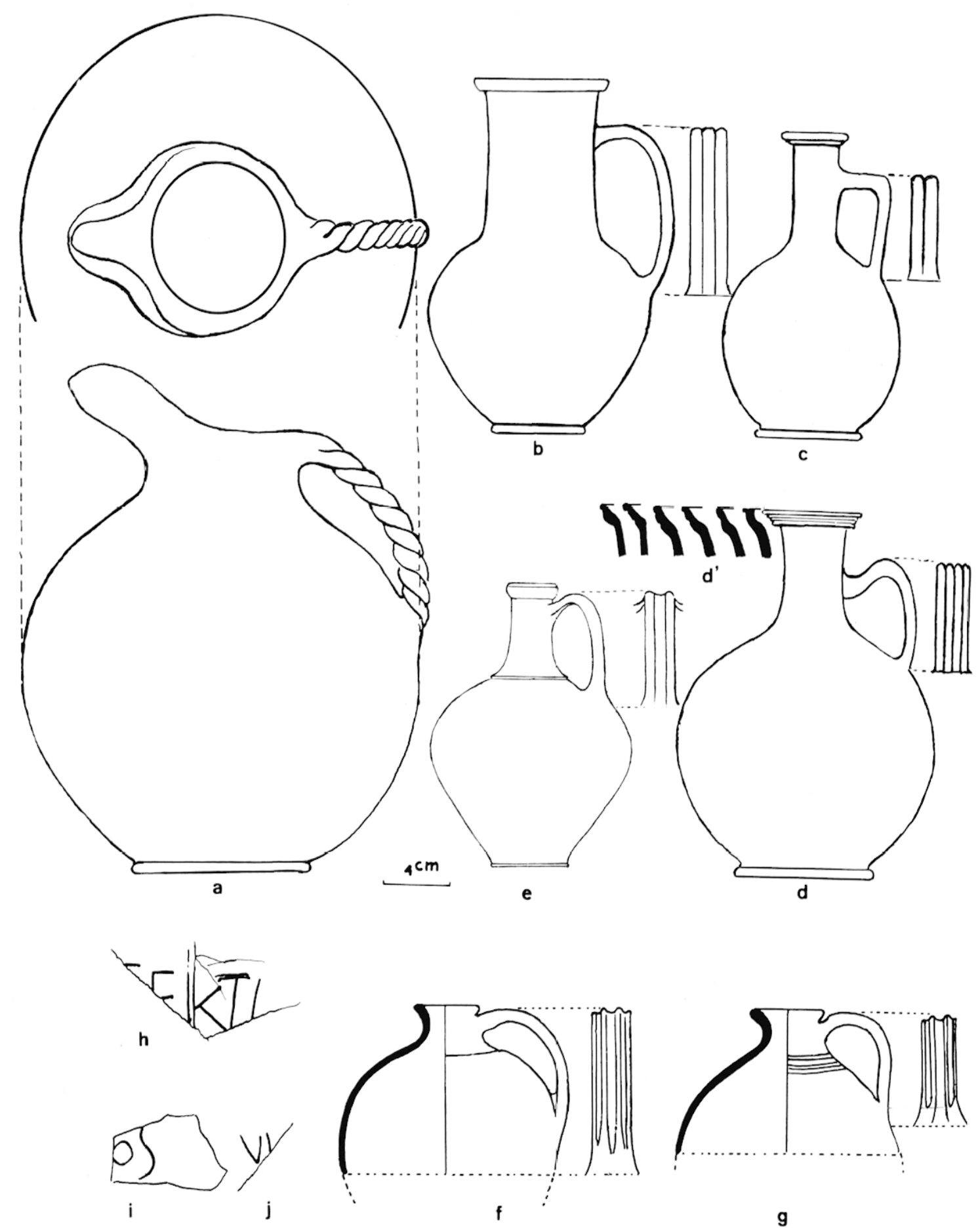

99. Puits $n^{\circ} 7$. Vases en poterie jaune el graflites.

IV. Polerie jaune.

Cette céramique, très abondante, constitue essentiellement des vases à liquides et des mortiers. L'argile est jaune clair, fine, homogène, tendre, sans engobe ni vernis, de coulcur identique à la cassure et à la surface. 
1. Vases a liquide ${ }^{42}$. Forme 1. Vase pansu a fond plat en bourrelet circulaire, col court, orifice prolongé en avant par un bec à bord abattu en dedans, et à l'opposé par une anse torsadée, caractéristique, se rattachant en haut de la panse. (H. : $28 \mathrm{~cm}$. D. (0.:9 cm. D. M. : $23 \mathrm{~cm}$.) (fig. $29 \mathrm{a})$. 37 fragments représentent 4 autres vases de même type dont un porte au col un nom gravé (fig. $29 \mathrm{~h}$ ).

Forme 2. Vase à panse globuleuse surmontée d'un haut et large col rylindrique, hord a levre évasée, anse trìs ouverte a 2 sillons longitudinaux joignant le $1 / 3$ supérieur du col au point le plus large de la panse (II. : 18,6 cm. I). O. : $8 \mathrm{~cm}$. D. M. : $14 \mathrm{~cm}, 7$ ) (fig. 29 b). 180 tessons proviennent. d'une trentaine de ces vases à liquide de différentes tailles.

Forme 3. Vase à panse sphérique, col élevé, étroit, bord à lèvre en double bourrelet d'inégale longueur, anse coudée ì une rainure médiane, pied large (H. : 15,8 cm. DM. : $11,2 \mathrm{~cm}$.) (fig. $29 \mathrm{c}$ ).

Forme 4. Vase à panse globuleuse, pied large, col élancé, anse flexueuse à 3 nervures verticales, livvre à 4 ressauls successifs décroissants. (II. : $18.4 \mathrm{~cm}$. D. M. : $16 \mathrm{~cm}$.) (fig. $29 \mathrm{~d}$ ). 14 fragments de bords de profils variés d'une dizaine de récipients (fig. $29 \mathrm{~d}^{\prime}$ ).

Forme ij. Vase a panse plus large que haute. col étroit élevé, anse à dépression verticale limitée par 2 sillons; livre en biseau, pied étroit (II. : $14,6 \mathrm{~cm}$. D. M. : $12 \mathrm{~cm}, 5)$ (fig. $29 \mathrm{e}$ ) et 4 autres fragments.

Forme 6. Grand vase it 2 anses, col court cylindrique, orifice à lèves plates (a pu être reconstitué gràce à un exemplaire complet trouvé dans la fosse-silo $n^{0} 2$ ( $(\mathrm{fig} .71$ a); quelquefois il y a un bourrelet a la base du col. Lne vingtaine de fragments de groulots de 5 récipients.

Dans cette série de vases a liquide, on peut inclure 2 parties supérieures de cruches en terre jaune rosée, a panse ronde, col court, et anse

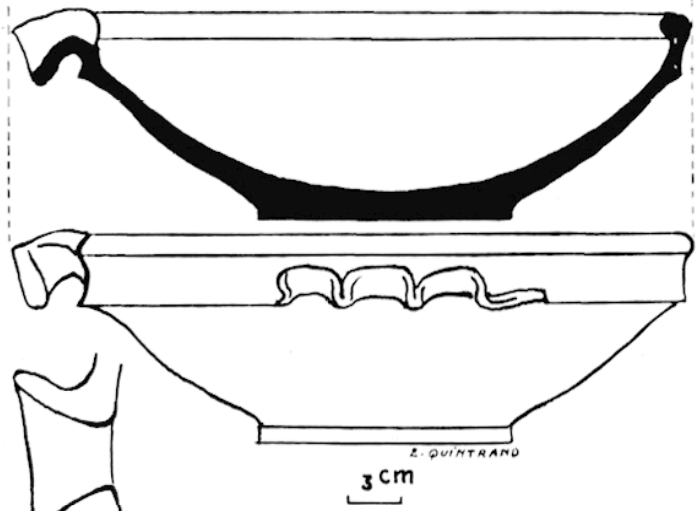

30. Puits n० 7 . Mortier en terre cuite. còtelée (fig. $29 \mathrm{f}$ et $\mathrm{g}$ ) ainsi qu'un fragment de tasse a 2 anses. de jalte à còté oblique et bords rentrants, et 2 fragments de couvercle a bouton de préhension. la forme.

Celte poterie jaune a fourni encore $1: 3$ vases a liquide incomplets, dont il est difficile de préciser

Les fragments sont nombreux : 2000 de parois, 44 de fonds plats, 1:00 de fonds a pied creux, 42 de bords. 6 d'anses simples, 25) d'anses à 1 sillon dont une parlie gravée a la base: 1 IS (fig. 13 f), 52 a 2 sillons, 14 a 3 sillons. 1 seul a 4 sillons et 1 anse horizontale appliquée en "oreille " c. graffites sur plusieurs tessons (fig. $29 \mathrm{i}$ j).

B. Morliers. Grands récipients à fond plat, épais, de forme tronconique renversée, bord un peu incurvé surmonté d'une lèvre munie d'un déversoir trapézoïdal curviligne et, de chaque còté, de 3 dépressions en are de cercle obtenues par le rabattement vers le haut de :3 languettes d'argile pour faciliter la préhension ${ }^{43} ; 2$ reconstilués en entier (H. : $10 \mathrm{~cm}, 5$. D. $0 .: 38 \mathrm{~cm}$ ) (fig. . 30$)$ et 70 ) fragments d'une dizaine de récipients identiques.

V. Poterie gris fer. 1. Vase ì liquide incomplet (type à bec trilobé, corps ventru et anse large). ( $\circ$ f. infra, puits $\mathrm{n}^{0} 10$ ) (fig. 56 a) 33 goulots et une quarantaine de fragments de 3 autres vases semblables. 2. 200 tessons de jattes et urnes ordinaires.

(42) "Olpi " des archéologues italiens.

(43) C'est le "vaso a ditate " rencontré dans plusieurs niveaux du Ier siècle av. J.-C. à Vintimille, X. Lambocilia, op. cil., p. 71 , fig. $29, \mathrm{n}^{\circ} 11$. 


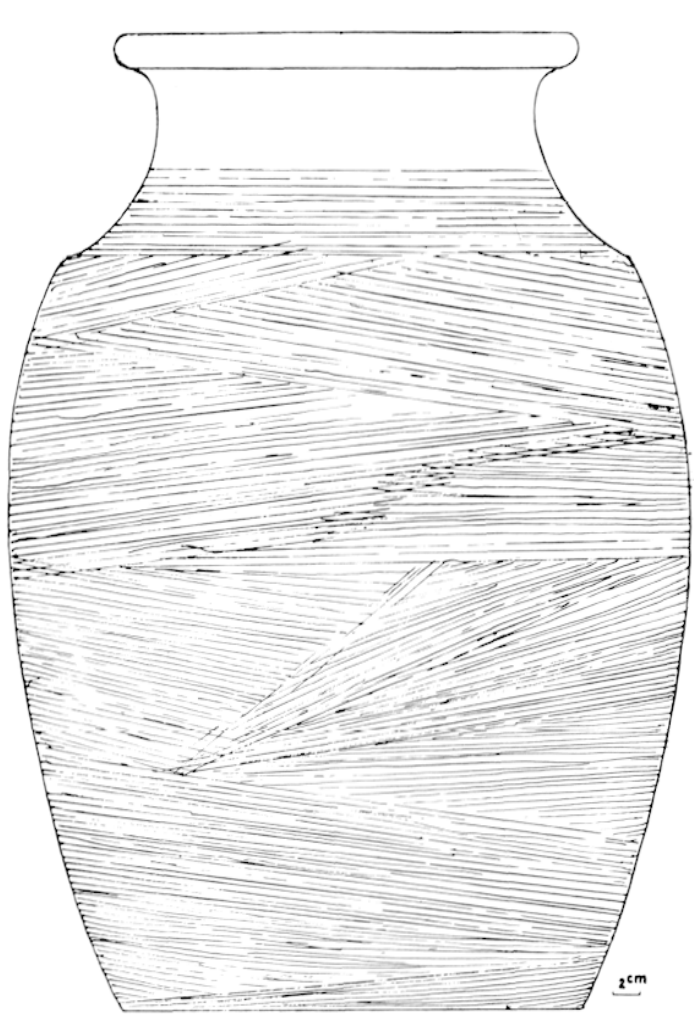

31. Puits no 7 . Cirande jarre.

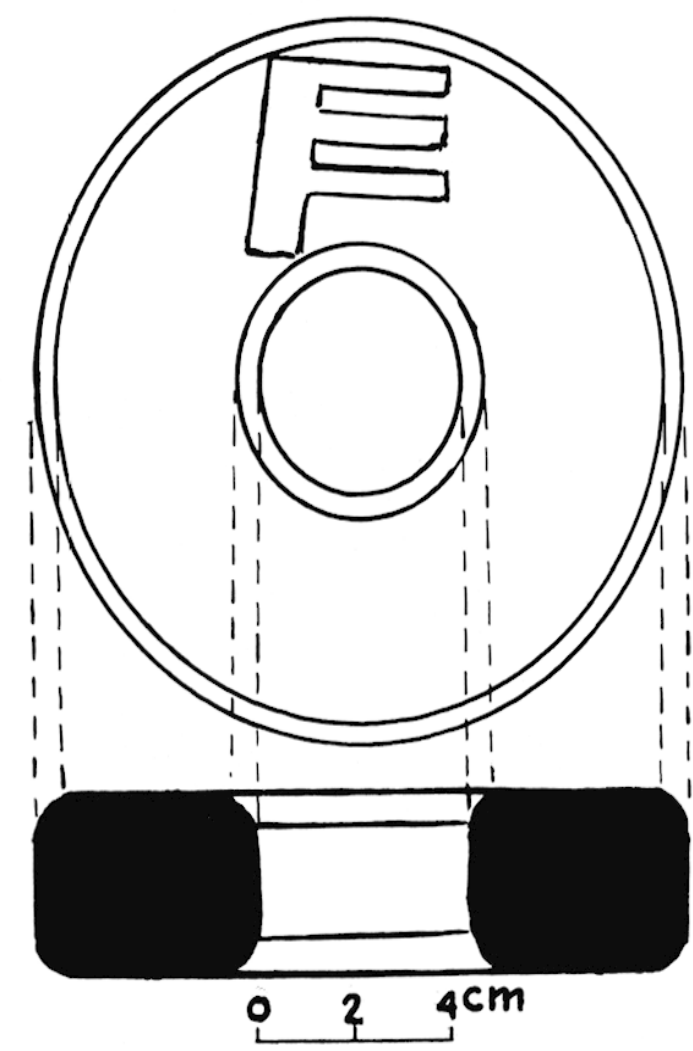

3:2. Puits $n^{\circ} 7$. Couronne en lerre cuite arec marque.

VI. Grande Jarre. Le plus grand récipient trouvé dans ce puits est une sorte de petit dolium en terre rougeàtre à grains de calcite, (reconstitué avec tous ses fragments : $11 .: 70$ cm. 1 ). $0 .: 32.6$ ('m. D. M. : 48 cm.) (fig. 31). Base d'un autre. genre pyllhos. à bourrelet, avec agrafe de plomb, vestige d'une réparation d'époque.

VII. Couronnes en lerre cuile. Elles sont presque loutes en argile jaune, finc (9 entieres ou recollées) ; une seule est en terre de dolium. Leur section est circulaire ou ovale un peu aplatie. 27 fragments représentent encore une dizaine de ces couronnes que l'on s'accorde à considérer comme des supports de vases. L'une porte un signe gravé avant cuisson (fig. 32) (4) $^{44}$ sur une autre on remarque l'empreinte d'une petite brindille.

VIII. Amphores. A noter la rareté de ces récipients représentés seulement par quelques tessons de type massaliote avec grosses paillettes de mica.

IX. Lampes. A. Type delphinoïde (f. Dressel 2) : 1. Lampe en terre grise à vernis noir brillant (campanien (i) à aileron latéral du còté gauche. Bord du réservoir ponctué de perles ; trou d'alimentation bien central avec petite perforation supplémentaire en bas à la périphérie de la cuvetle: bec lisse large, rectiligne à son extrémité, trou pour la mèche largement ouvert, anse rubanée à bourrelets latéraux; sous le réservoir : lettre $M$ à bouts cerclés, en creux (H. : $3,5 \mathrm{~cm}$. I. : $12 \mathrm{~cm}$.) (fig. 33 a)..$^{45}$.

14) Signe identique sur une couronne de la Coll. Gilles, au Musée des Apilles à Saint-Rémy-de-Provence.

(45) Marque signalée par R. BAIl.I.Y, Essai de classificalion des marques de poliers sur lampes en argile dans la Varbonnaise dans Cahiers ligures de Préhisloire el d'Archéologie, II, l' partie, 1962, p. 112 et p. 111, no 18, figuré fautivement (lettre $\mathrm{N}$ au lieu de $\mathrm{II}$ ); lampe mentionnée par F. Bwort, L'épave du Grand Congloué, p. 112 et 113. 

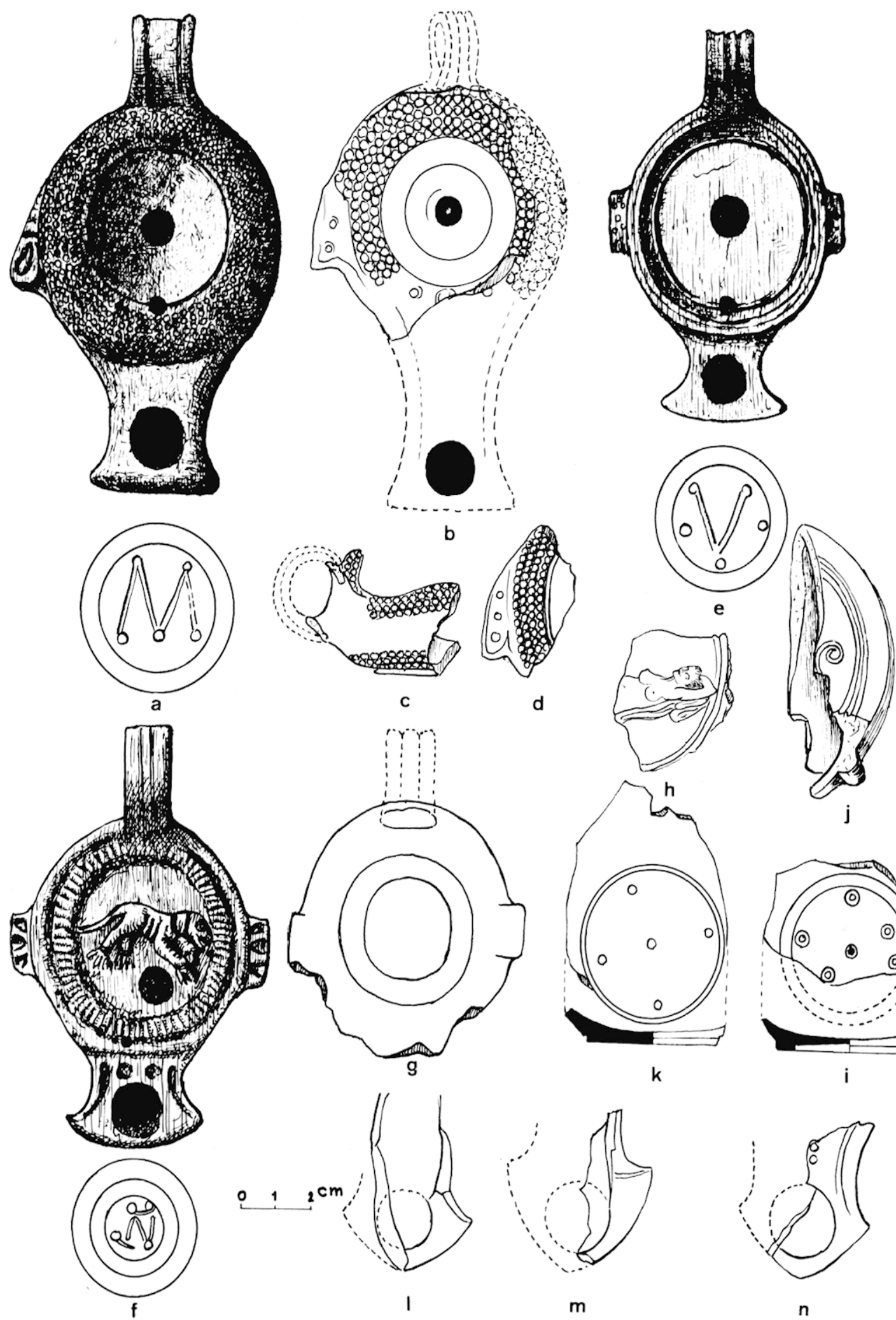

33. Puits $n^{\circ} 7$. Lampes en terre cuite. 
2. Lampe incomplite en argile jaune à vernis marron clair ; réservoir granulé à cinq rangées de perles autour d'un disque lisse a l trou rentral, avec aileron, a deux perles sur le dessus (fig. 333 b) ${ }^{46}$. el d).

:3. Fragments de 2 aut res lampes de mème type (partie postérieure el aileron is :3 perles) (fig. 3)3 c

B. Type a ailettes (f. Iressel 3) : 1. Lampe en argile marron clair a vernis foncé. Itessus plat avec trou d'alimentation bien au centre, el un $2 \mathrm{e}$ plus petit, en bas. dans son axe longitudinal. De. chaque còté du réservoir, protubérance rectangulaire parsemée sur le dessus de petils points creux. Bec large. un peu arqué sur le devant, avec grand trou pour la morche, anse rubanée à 2 sillons:

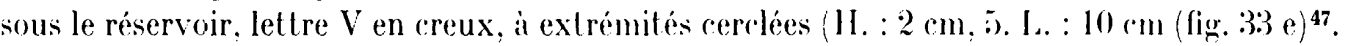

2. Lampe en terre jaune a vernis bistre. bec arrondi au bout, incurvé sur les coltes ; bords soulignés par 2 petits sillons arqués, préfiguration de volutes, qui doublent la courbure; a la rarine du bec, entre leurs extrémités : 2 petits cercles. Le dessus, circonscrit par un bandeau circulaire st rie est orné d'un quadrupide couranl a droite. sous le pied. lettres en creux. a jambages cercles à leur extrémité, entourées de 2 rercles concentriques (H. : :3 cm. L. : lo.5 (m.) (fig. 3:3 f) f8

3. Parlie postérieure de lampe en argile rougeattre it engobe plus foncé. avec départ d'anse (fi $\underline{x .}$. 3:3 $\mathrm{g})$.

4. Deux fonds de lampes : l'un, plal. circulaire avec 5 petits rercles disposés en rroix (fig. 3:3) h. l'autre à pied bas. ponctué de 6 petits cercles ( $(\mathrm{igg} .333 \mathrm{i}$ ) se rattarhent au lype ì ailettes.

c. Type a volutes latérales: 1. Fragment droil d'une lampe en lerre cuile grisàtre à engobe marron, dont on ne voit de l'ornementation qu'une tige enroulée (fig. .3:3 j).

2. Fragment de dessus orné : femme nue couchée, à droite le bras gaurhe replie sous la lithe (argile blanche sans engobe), (fig. $333 \mathrm{k}$ ).

I). Divers (fig. :3:3 I m n I. 5) becs incomplets : 4 angulaires, 1 en "ogive ». 4 anses striées ("l une vinglaine de fragments.

X. Bronze. Petile fibule a charnire, arc is courbure réguliere, ètroit au porte agrafe, qui est surmonté d'une courte tige transversale. s'élargissant progressivement jusqu'i son extrémité opposée. La fixalion de l'ardillon à la lête de l'arc est assurée par une petite clavelte en fer. (H. : $1.7 \mathrm{~cm}$. L. : 4 cm.) (fig. 34 a). Ce type de fibule caractéristique de la Téne III, a été l rouvé à Mlésia, Bibracte. dans l'enceinte de l'oppidum de Pommiers (Aisne), el à Ensérune ${ }^{49}$.

Pelil anneau filiforme ferme (I). : $1,5 \mathrm{~cm}$.) (fig. $34 \mathrm{~b}$ ), aiguille it chas $(\mathrm{L}: 8 \mathrm{~cm}$.) (fig. $34 \mathrm{~d}$ ). tige termince par un crochet, fragments d'un bracelet a tige plate unie, 2 plaquettes arrondies perforées à chaque extrémité (fig. . 34 c), 8 fragments d'un miroir rectangulaire mét allique, et 16 débris indéterminables.

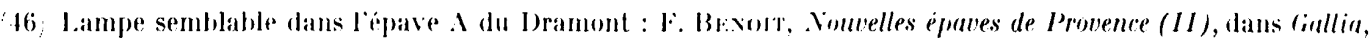
N1III, 1960), p. .22, fig. 21 .

17. K. Bamis. op. ril., p. 80 et p. 85, fig. 1.

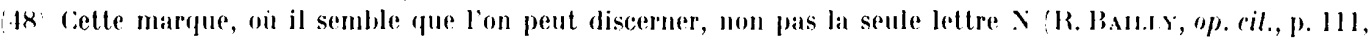
ligr. 17 et p. 112) mais plutiot les 3 lettres 1. V. I., s'est rencontre sur 2 lampes à 2 becs du ger $^{\text {s. ar. }}$. J.-(.. cf. H. B. Waltrirs, Calalogue of the Gireek and Roman lamps in the British .Museum, 1914, p. 6x, nos 46.1 et 165$)$.

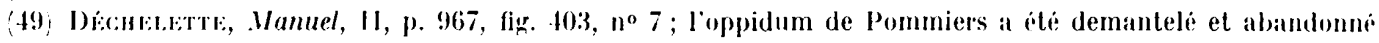

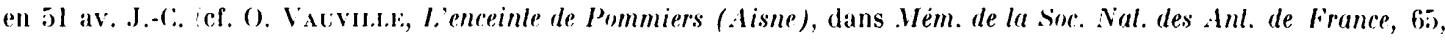

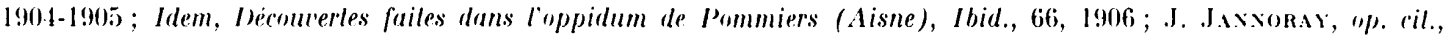
pl. I.IX, 1 . 
XI. Fer. Plusieurs maillons d'une chaine (fig. $34 \mathrm{e}$ f), 58 clous et une cinquantaine de morceaux informes, très oxydés.

XII. Os. Deux épingles, ou styles, ì corps fuselé allongé terminé par une pointe conique (fig. 35 a, b), pointe triangulaire a 2 rainures en angle aigu au bout d'une tige a encoche a la base (fig. 35 c). Plaquette en forme de parallélogramme perforé à une extrémité, dont la surface porte 2 rayures parallèles dans le sens de la longueur (fig. 3ij) d). Fragment de tige cylindrique. Os plat de bouf à bordure dentelée due a l'enlevement de rondelles avec un emporte-pièces (fig. 35 f), fragment de rouleau cylindrique.

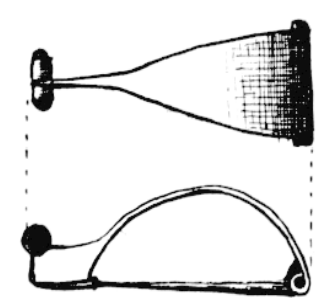

a
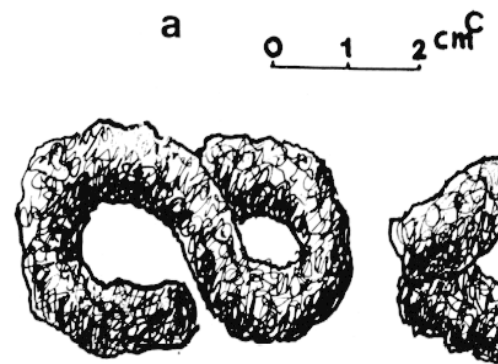

e

34. Puits no 7 . Bronze et fer.

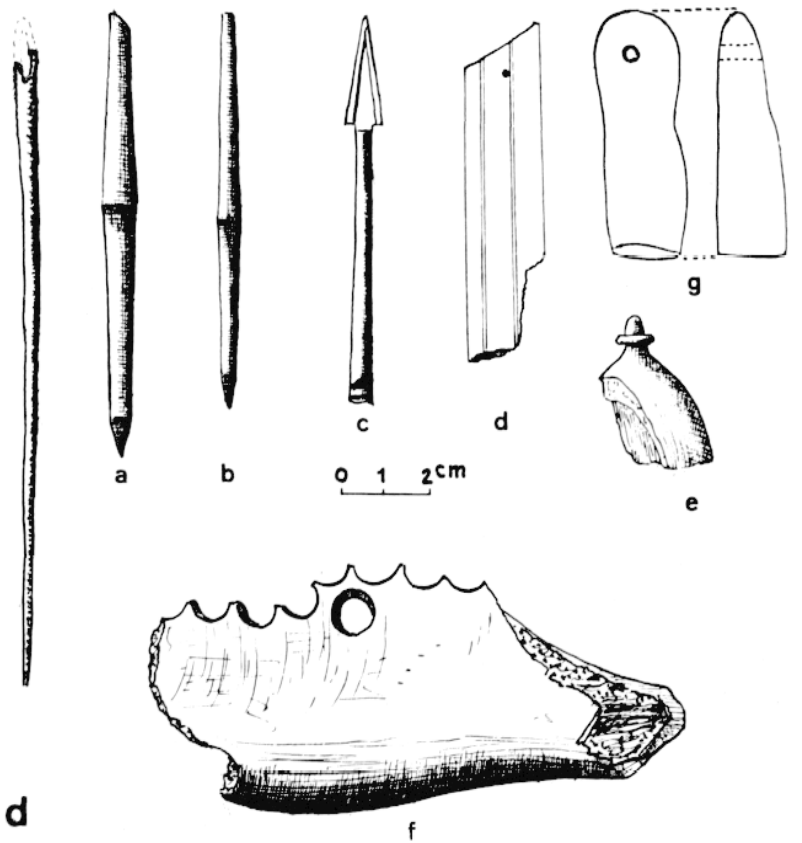

35. Puits $n^{\circ} \bar{Z}$. Os travaillé et objets divers.

XIII. Divers. Fragment de petit récipient en albàtre de forme conique, avec bouton terminal (fig. 35 e), de bras de poupée (?) en terre cuite (fig. 35 g).

XIV. Faune. Yombreux os de bovidés, ovidés, et suidés, valves de moules et huitres.

Si la couche supérieure montre que la fin du remplissage de ce puits s'est eflectuée dans la $2^{e}$ moitié du ${ }^{\text {er }}$ s. ap. J.-C., la partie inférieure indique un comblement progressif qui a commencé vers le milieu du ${ }^{\mathrm{e}}{ }^{\mathrm{r}}$ s. av. J.-(.. et a été surtout important pendant la 2 e moitié de ce siècle (fig. 36).

Nous allons maintenant décrire les autres puits, en suivant l'ordre chronologique. 


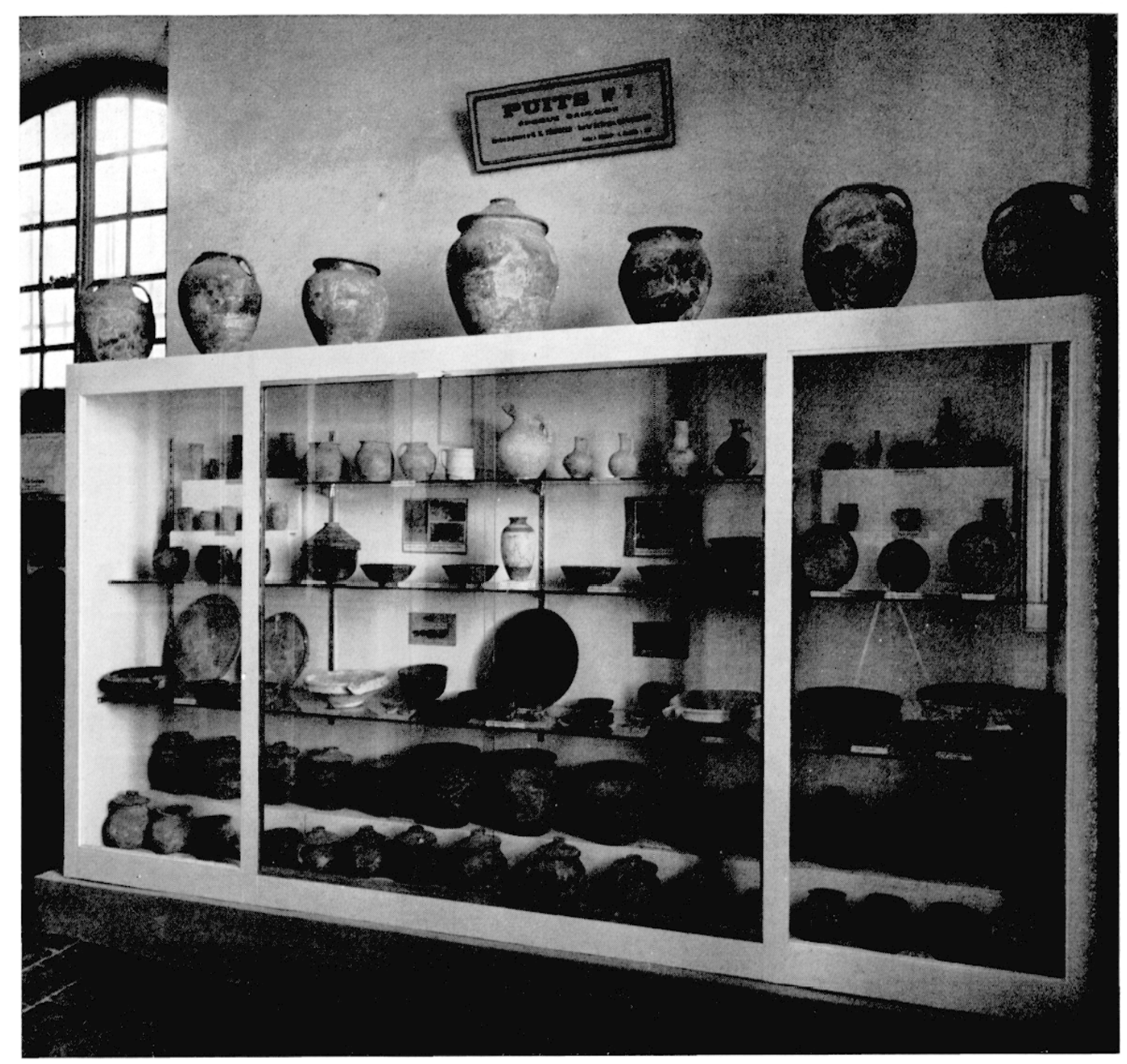

36. Vitrine du puits $n^{\circ} 7$ an Vuspe de Cavaillon.

\section{PIITS $x^{0} 1$}

Il a été découvert en 1936 dans la carrière de .I. A. Jeune. La colonne de terre qui le remplissait sur environ $6 \mathrm{~m}$. el s'était éboulée sur le front d'exploitation a donné de nombreux tessons typiquement hallstattiens accompagnés de quelques rares fragments de poterie d'importation.

I. Céramique. 1. Polerie fine d'importalion. 1. Plocéenne tardive : a) Pàte grise tendre: fragment de pied profond de coupe à parois très épaisses (fig. 36 bis a).

b) Pàte grise dure à engobe foncé : fragments de bord évasé (fig. 36 bis d) de panse carénée, avec zone ondée sur 4 sillons parallèles (firg. 36 bis e), de panse carénée avec stries horizontales (fig. 36 bis b) et 6 tessons informes.

c) Pàte grise dure à engobe clair : fragment de pied oblique (fig. 36 bis b) et de bord de skyphos avec départ d'anse de section circulaire (fig. 36 bis c). 
2. Attique : Fragment de panse de vase, probablement un lécylhe, peinte en noir sur fond jaune foncé. Le motif ornemental réliculé se compose de bandes curvilignes rayonnant aulour d'un rercle pointe (fig. $3(\mathrm{i} \text { bis i })^{50}$.

3. Précampanien : Pied de vase en argile fine, dure, rosaitre, à beau vernis noir brillant, orné a l'intéricur de 4 palmettes de bon slyle. composées de 7 pétales avec a la base les 2 volutes qui caractérisent la palmelte arrecque ${ }^{51}$. La cassure régulière tout autour, et la présence de 2 trous c'entraux opposés de chaque coté de ce pied, font penser à son utilisalion romme roulelte (fig. 36j bis g).

4. (iampanien 1 : a) Fragment d'anse à poucier, probablement d'une roupe dans le genre de celles trourées sur l'oppidum des Pennes ${ }^{52}$ (fig. :36 bis j).

b) Fragment de pied de bol. brisé a la bordure d'une palmelte intérieure centrale (fig. 36 bis h).

B. Polerie indigène. 1. Lirnes. 3ätj Lessons de vases du type olla a bord oblique. evasé, panse arrondie et fond plat. en lerre cuite rrossière de couleur marron noiritre, contenant des grains de calcaire el parfois de pelits oolithes d'oxyde de fer, se rapportanl it une quarantaine de récipienls.

La paroi externe, dont le peignage oblique irrégulier exclut l'usage du lour, est ornée i sa partie supérieure de motifs géométriques imprimés avant cuisson. Il faut noter la variélé de terhnique du décor obtenu, soit ì l'aide d'un batonnet (ondulalions simples, lignes brisées disposées en chevrons parfois surmontés d'un sillon, ou rombinaison de rhevrons et traits obliques) (fig. 37 a) soil par l'empreinte des dents d'un peigne de potier (chevrons
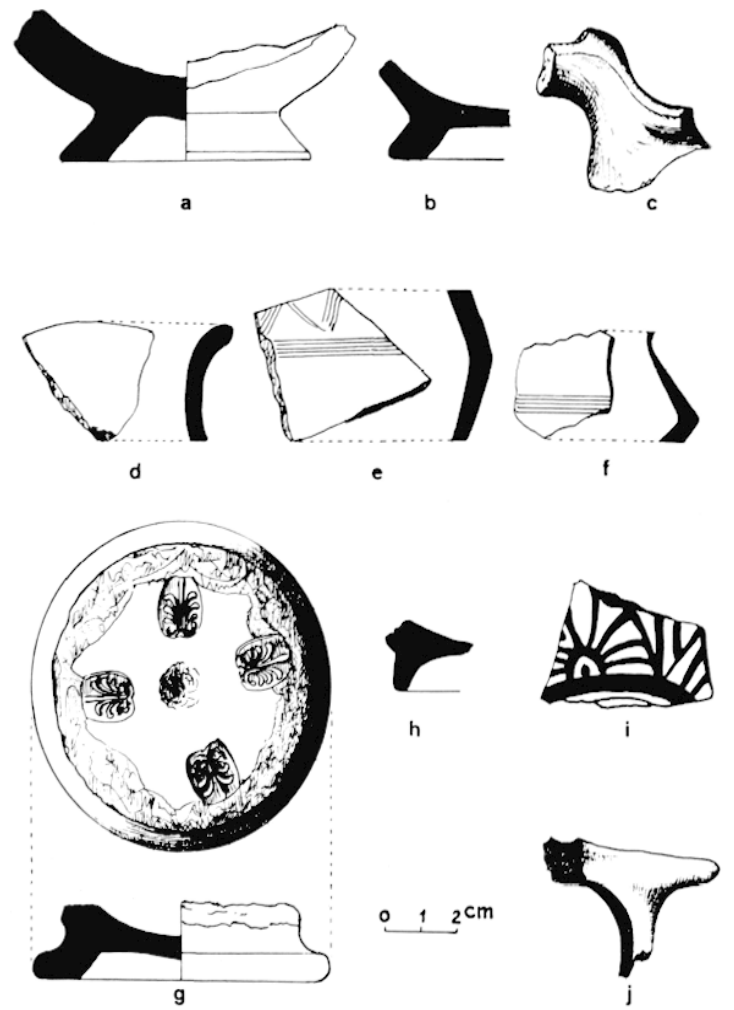

36 his. Puits no 1 . Poterie phocenne et attique. simples ou traits allongés obliques sous sillonj (fig. $37 \mathrm{~d}$ ), soil par impression d'une coquille de gastéropode lerrestre placée obliquement (empreintes fuselées de clausilia ou plus larges de cyclostoma ou de rumilla) (fig. 37 b) $)^{53}$.

Cing vases ont pu ètre reconstitués en entier.

a) Lrne ornée d'une suite de chevrons sous un sillon circulaire.

(II. : 2: cm. I). (). : 18 (m.) (fig. 38 a).

(5) Voir lornementation i urande palmette sur la panse des lécythes de la nocropole Martis, d'Ampurias :

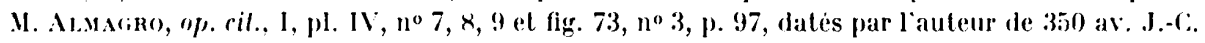

(j) P. E. Conвıтt, Palmelle slamps from an Allic black glaze uorkshop, dans .Mesperia, XXIS, 1955, pl. $66-71$.

(52) F. B1:Nort, L'épave du Cirand Congloué, p. 81, pl. V', fig. b 2 et 3.

(53) Cette particularití de decor sur ollae, a éti remarquee dans plusieurs grisements de l'A gre du fer de l'rovence. II. de Gírix-Racaro, Empreinles cuquillires sur des poleries de la Tène II, dans Rhodania, Congris de Cannes-(irasse, 1929 , p. 87 à 89 . 

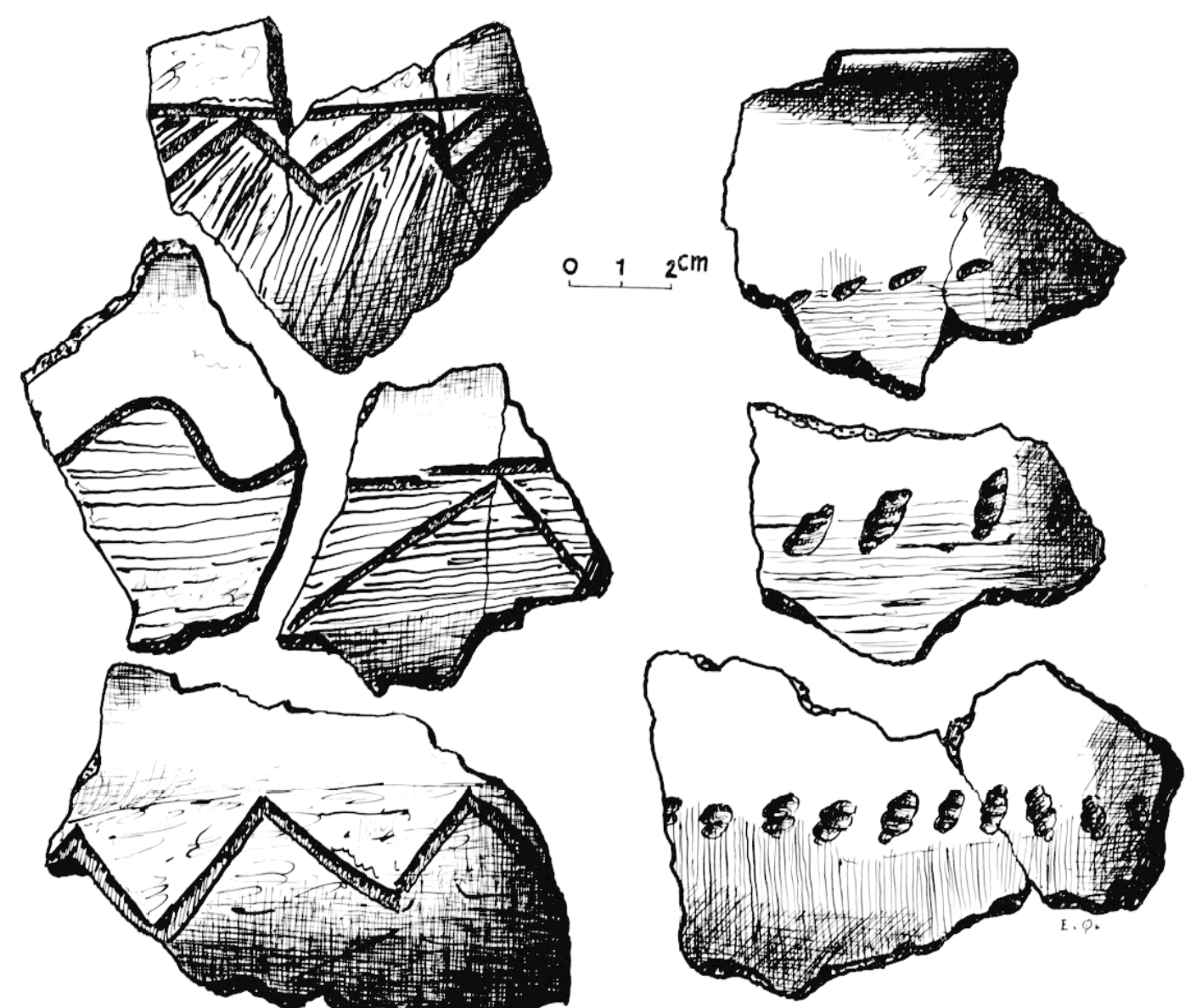

b
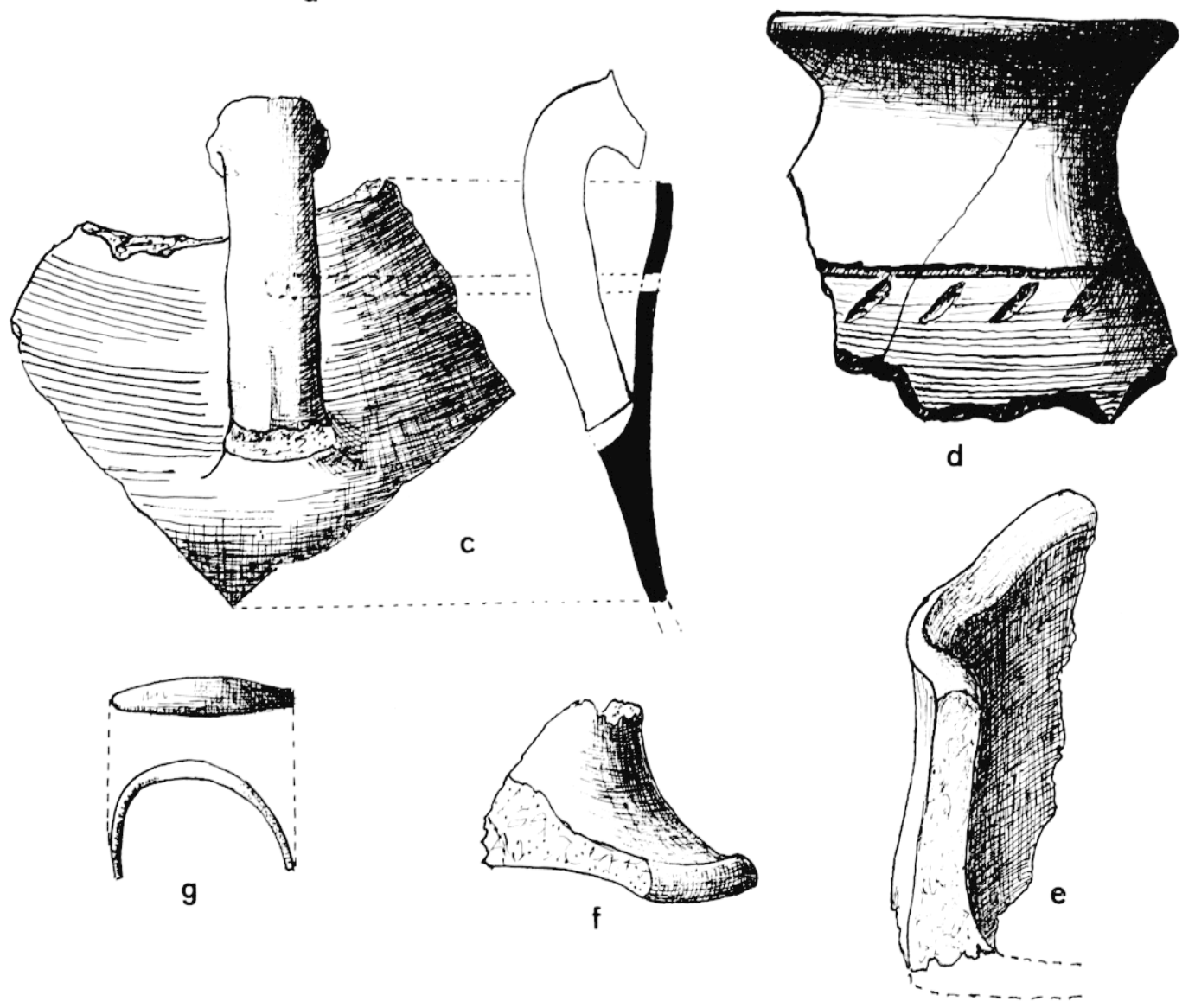

37. Puits no 1. Céramique indigrene. 

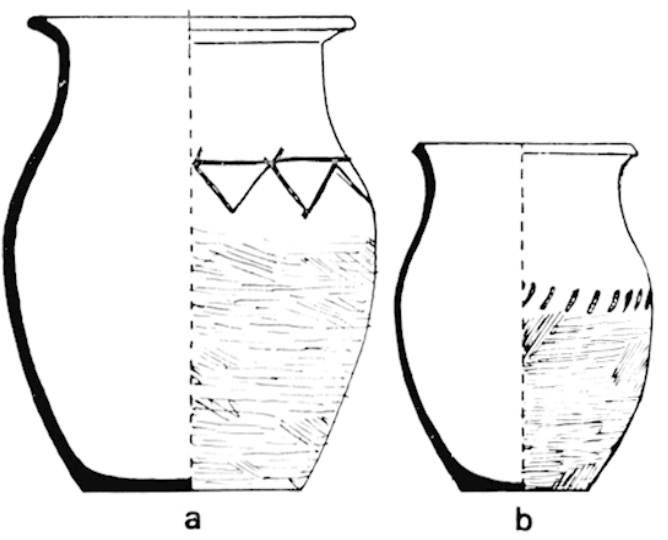

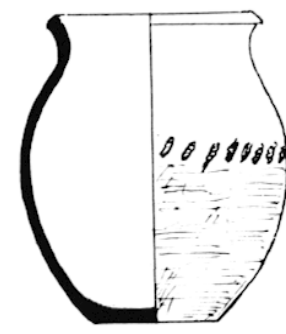

C

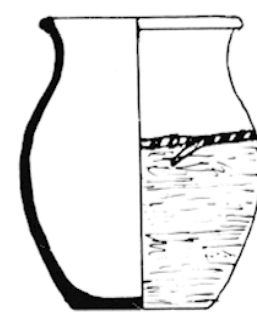

d

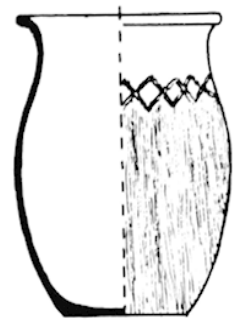

e
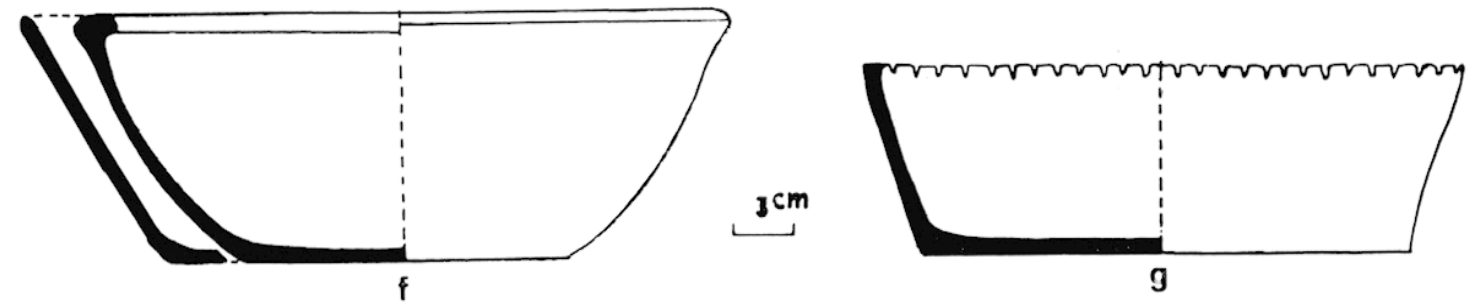

3x. Puits nO $^{\prime}$ 1. Vases indigènes.

b) Lirne de forme régulière, avec rangée d'encoches obliques à intérieur striè (H. : $16 \mathrm{~cm}$. I). O.: 12 ('m.) (fig. $38 \mathrm{~b}$ ).

c) Urne de mème lype ornée de fines incisions presque verticales (H.: $17 \mathrm{~cm} . \mathrm{D}, 0 .: 13 \mathrm{~cm}$.) (fig. 38 c).

d) Crne ì décor constitué par une bande irrégulière horizontale ponctuée d'encoches obliques (II. : $15 \mathrm{~cm}$. I). $0 .: 11 \mathrm{~cm})$ (fig. $38 \mathrm{~d})$.

e) Urne ornée de chevrons opposés en sens inverse el entremélés, panse étroite peignée verlicalement (H. : $14 \mathrm{~cm}$. D. (). : $11 \mathrm{~cm})(\text { fig. } 38 \mathrm{e} \mathrm{e})^{54}$.

Cn seul tesson d'urne porte une anse simple avec trou de raccomodage dans la paroi (fig. 37 c).

2. Vases carénés. Écuelle surbaissée, apode, à bords évasés, panse arrondie à carène haute; fond à ombilic central en dessous. L'argile est marron, assez fine, bien lissée et lustrée sur toute sa surface. 6 fragments se rapportant ì 3 de ces récipients. Un $4 \mathrm{e}$ a été reconstitué en entier. $(\mathrm{H} .: 4 \mathrm{~cm}, 7$. D. 0. : $11,7 \mathrm{~cm}$. D. B. : :5.5 cm.) (fig. 39 a) $)^{55}$.

3. Bols. Sortes de coupes en forme de calotte sphérique, à bord rentrant el pied creux en poterie semblable aux vases carénés précédents, qu'ils accompagnent aussi dans les cimetières marniens ${ }^{56}$.

(5.) Des urnes de mime tỵpe avec decor similaire se sont rencontrées a Einserune (Hérault) : .I. Lot:s, La polerie grossière du I er Age du Fer à Enserune, dans Revue d'èludes ligures, XVII, 1951, p. 10I, fig. 4, nos 20 à 37, et

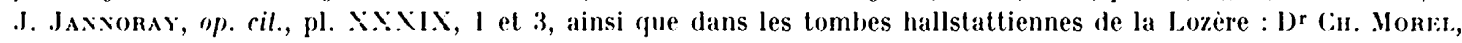
Siepullures lumulaires de la région du Freyssinel (Causse de Sauvelerre), 1936, p. 71, pl. 111, forme 10.

(55) Dr (.н. Монеi, op. cil., p. 69, fig. 17 ; P. Favret, Les nécropoles des Jogasses a Chouilly (.Marne), dans Préhistoire, $\mathrm{v}, 1936$, fig. $43, \mathrm{~N}^{\circ} 106$

(56) P. Favret op. cil., fig. 44, $1^{\circ} 22$; 1)r Cil. Monel., op. cil., forme 7, p. 70, fig. 18 ; M. Lor Is, op. cil., p. 99, fitr. $3, n^{0 *} 25$ à 31 . 
52 fragments d'une douzaine de ces récipients dont l'un a pu ètre restitué (H. : $8.6 \mathrm{~cm}$. I). (). : $16,3 \mathrm{~cm}$. I). B. : 5,2 cm.) (fig. 39 b). (Certains de ces bols dont les bords sont rectilignes ont dû servir de couvercles d'urnes.

4. Jalles. a) Grand récipient de $35 \mathrm{~cm}$. de diamitre (reconstilué à moilié) en forme de curette a panse lisse et fond plat. en argile marron lustrée, bord rentrant a levre épaissie, un peu arrondie sur le dessus. ( $\mathrm{H} .: 11 \mathrm{~cm}$.) (fig. $38 \mathrm{f}$ ). Fragment d'une autre i paroi oblique el un bord avec pincement dans la paite pour faciliter le versement (fig. $37 \mathrm{e}$ ).

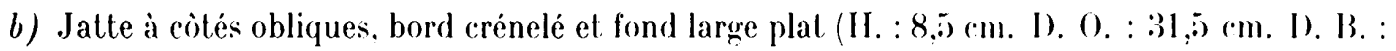
$26 \mathrm{~cm}$.) (fig. $38 \mathrm{~g})$.

Parmi les tessons divers signalons un pied de coupe en argile marron lissie (fig. $37 \mathrm{f}$ ).

II. Bronze. Ire de fibule courbe mince élargi au milieu, brisé de chaque còté (I. : :3. jorm. (figr. $37 \mathrm{~g})$.

III. Fer. Fragment de tige de clou tris rouille.

IV. Fanne. Canon de cheval, défense de sanglier, os longs de bovides el ovides. helix.

PliTs $N^{0} \stackrel{2}{2}$

Carrière Thiébold (avril 1941). La terre contenue dans la partie supérieure de ce puils a donné de la poterie réduite en petits fragments, accompagnée d'unc moilié de meult. en mollasse et de 4 rouronnes en argile jaunàtre fine à trou central régulier. Meux ont Ir.

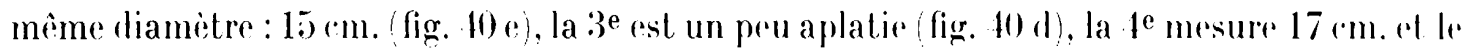
trou 6 ('m.

I. Ceramique. 1. Polerie fine:

1. Campanien el imitation. Tesson d'écuelle en Camp. I. fragment de bord d'ereuclle. imitation Camp. a vernis marron mélallique, fragment de bord de plateau. imitation lamp. C.

2. Poterie orangée claire à petits points brillants de mica. enduit plus fonce : fragment de bord de bol a sillon pries de la livere (fig. 40) a).

3. Pré-sigillée. Fragment de bord de coupe (lig. 40 b).

4. sigillée ilalique. Fragment d'assielle, de panse de coupe de de pied de bol aver leminaison de nom de potier : ... E.XT (X el T liés) (fig. 40$)(\cdot)$

B. Polerie commune:

1. Fragments de cols, panses peignées, el fonds d'urnes de tradilion hallstatlienne.

2. Fragments de jalte lisiée a fond ombiliqué. ol de pied de bol (lypes du puils precédent).

3. Fragments d'ollae de la Tine.

4. Fragment de bord caréné de jalte basse type à goulol court du puits no 7 ).

\section{Faune.}

Os de bovirlés, ovidés et suidés.

Ces vestiges sont à classer, en gros, à la Tène III, et à une époque où persistent des types hallstattirns altardés. 


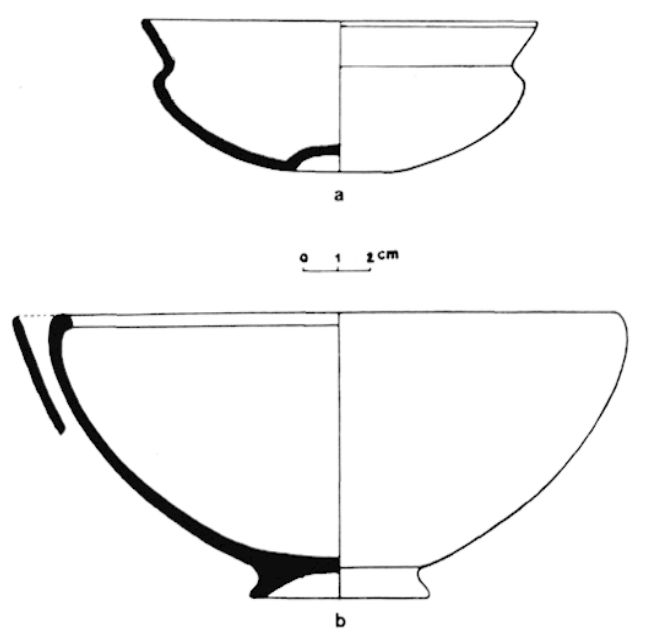

39. Puits no 1. Coupes en terre cuite Iustrice.

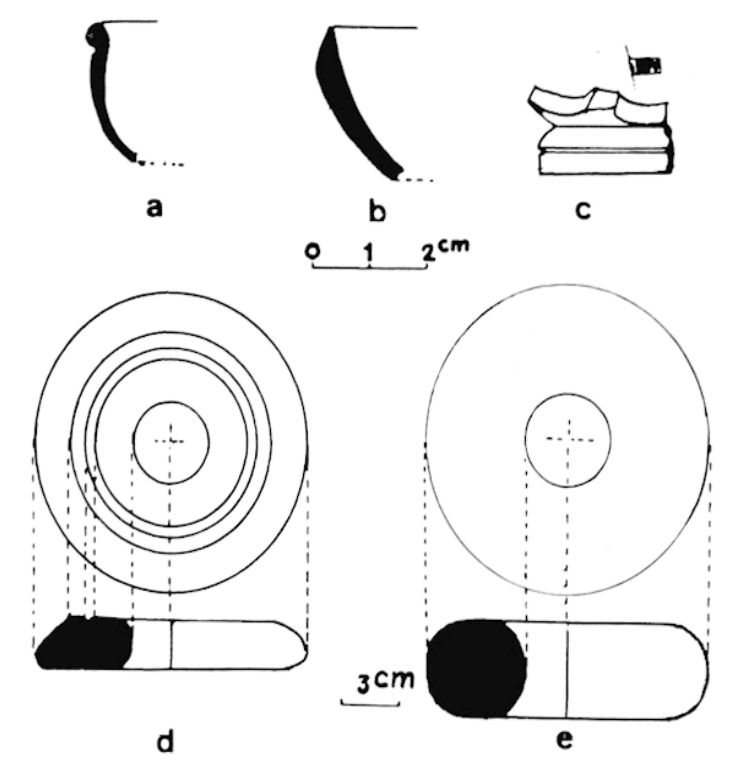

40. Matériel du prits no 2.

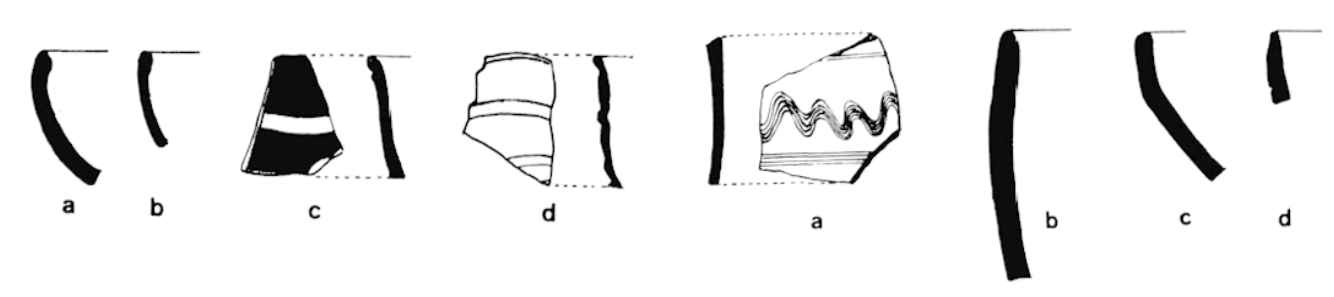

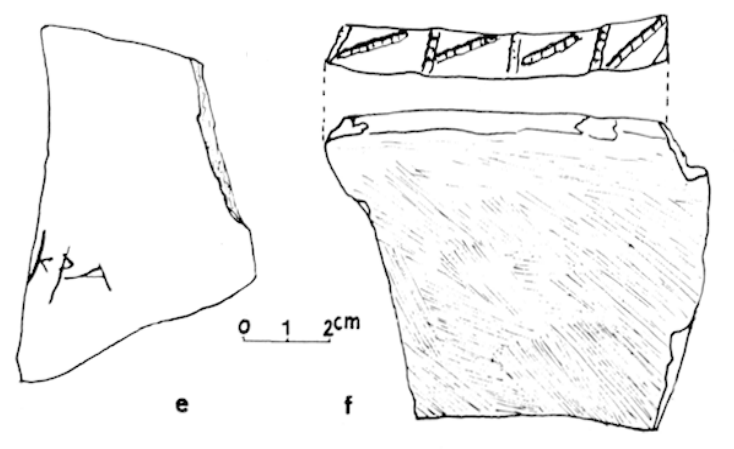

11. Céramique du puits $n^{\circ} 3$.

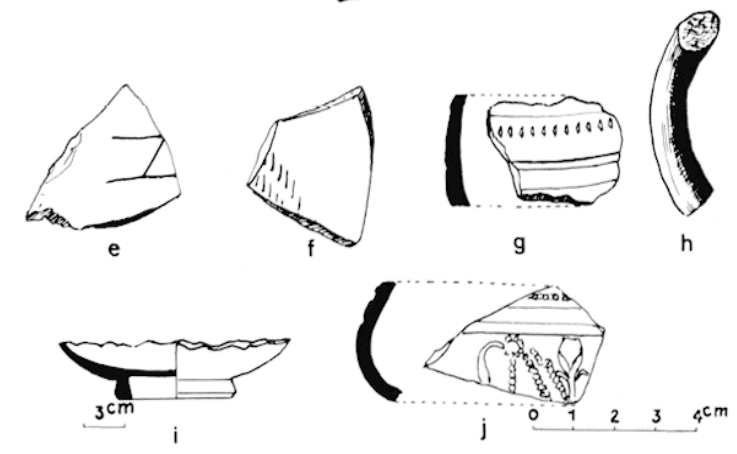

42. Puits $n^{\circ}$ 4. Ciramique phocienne, campanienne et sigrillee.

PUITS $\div 03$

Carrière Thiébold (mai 1941). Il se trouvait à $8 \mathrm{~m}$. environ à l'est du puits $\mathrm{n}^{0}{ }^{2}$. Comblé par une terre grisître très tassée avec par endroits des brindilles de charbon de bois et des plaques d'argile, il contenait des poteries très fragmentées, de même époque que le précédent.

I. Céramique fine. A. Proto-campanien : Une dizaine de fragments de coupelles à pâte claire et vernis très brillant (fig. 41 a). 
B. Campanien : Fragments de bords et panses de coupes et écuelles en Camp. A (fig. 41 b), de bol de teinte marron avec bande peinte blanchàtre intérieure (fig. $41 \mathrm{c}$ ), d'écuelles en imitation Ciamp., de paroi de patère avec partie terminale d'un graffite fail au poinçon : ....KPA (fig. $41 \mathrm{e}$ ). 6 fragments de plateaux et patères en Camp. C, 4 fragments de coupes en terre jaune à vernis marron écaillé (imitation Camp. tardif).

C. Pré-sigillée : fragments de panses de bols en terre jaune à couverte brun clair.

$D$. Vases à parois minces : 6 fragments d'un vase en argile grise dure avec 2 petits bourrelets parallèles près du bord (fig. $41 \mathrm{~d}$ ).

II. Céramique commune. 92 tessons d'urnes de type hallstattien avec encoches au col et bord évasé -- anse allongée à sillon vertical profond - 3 fragments de coupes lisses à pied creux - fragment de jatte à parois obliques portant sur le plat du bord une série d'encoches cannelées compartimentées (fig. 41 f), fond ombiliqué de vase carené lisse (cf. puits $\mathrm{n}^{0} 1$ ).

III. Poterie jaune. 46 petits tessons dont 6 anses appartenant à des vases à liquide (formes variées du puits $n^{0} 7$ ).

IV. Faune. Os de porc (mâchoire), mouton, bouf et cheval.

\section{PUITS No 4}

Carrière Thiébold (juillet-août 1941). Son ouverture mesurait 1 m. 70 de diamètre, et la portion fouillée, dégagée verticalement sur 5 mètres, allait en s'évasant vers le haut. Il contenait plusieurs gros mocllons en mollasse blanche, quelques-uns moulurés, des fragments de legulae, des plaques de béton et d'enduit muraux ainsi qu'un important matéricl archéologique que nous allons décrire ci-après.

1. Céramique fine. 1. Phocéen : un seul fragment de paroi de vase peu épaisse, en terre grise fine, dure, à couverte gris foncé orné d'une série de traits ondés irréguliers, entre 2 bandes rectilignes (fig. 42 a).

B. Campanien : petits fragments de coupes et bols à bord arqué ou légèrement coudé, en Camp. I (fig. $42 \mathrm{~b} \mathrm{c} \mathrm{d}$ ). Tesson avec graffite (fig. $42 \mathrm{e}$ ). Fragments de plateau à double rangée d'incisions, de panse, avec rang d'encoches et anse en Camp. B. (fig. $42 \mathrm{f} g \mathrm{~h}$ ). Fond de bol et fragment de panse en Camp C.

C. Imitalion de Campanien : pied creux d'une grande écuelle en argile jaunàtre à vernis marron clair (fig. 42 i).

D. Sigilléc : $1^{0}$. Vases ornés. I part un petit tesson de vase forme Drag. 29 (haut de panse avec sautoir et motif floral (fig. 42 j) c'est le bol hémisphérique (f. Drag. 37) le plus représenté.

a) Bol incomplet; le décor comprend de haut en bas : une couronne d'oves à tête du dard étoilée, un cordon de figures pectiniformes entre 2 lignes perlées, une guirlande de boutons fusiformes trifoliés, enfin au soubassement, un cordon chevronné sinistrogyre (provenance probable : ateliers rhénans) (fig. 43 a). ${ }^{57}$.

(57) R. Cagiviat et V. Chapot. Manuel, II, p. 456. 

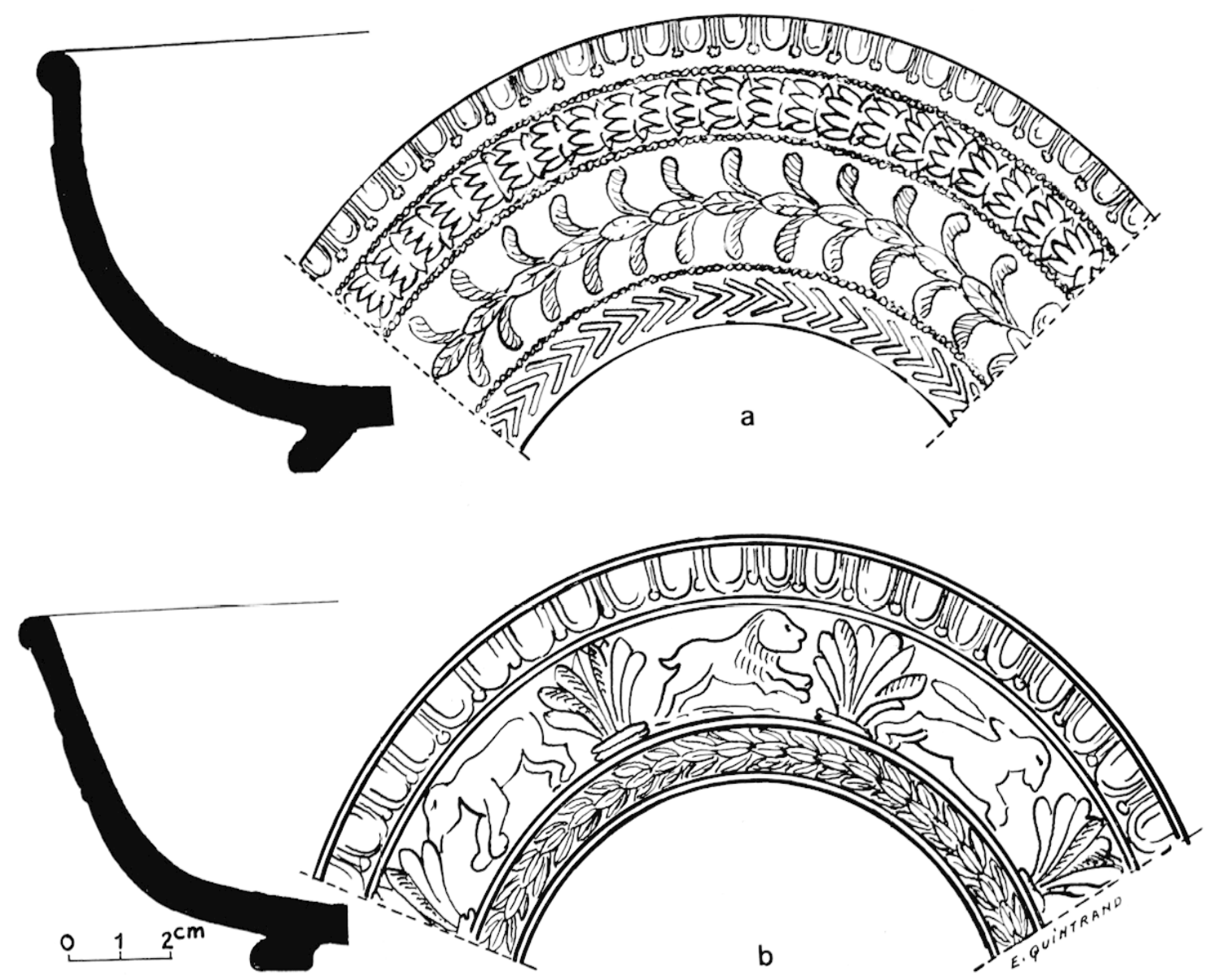

43. Puits $n^{\circ} 4$. Vases sigillés ornés.

b) Bol incomplel. Décor flou. Sous le couronnement habituel d'oves, animaux bondissant (lions) alternant avec une toufle de feuilles en éventail. Au-dessous, guirlande trifoliée sinistrogyre (provenance probable : ateliers rhénans) (fig. $43 \mathrm{~b}$ ).

c) Bol incomplet. Décor à panneaux simples représentant des oiseleurs accroupis, marchant ou immobiles au milieu d'oiseaux divers, séparés par une tige verticale renflée et trilobée à l'extrémité supérieure (la Graufesenque, époque Néron-Vespasien ${ }^{58}$ (fig. 44 a).

d) Fragment de panse décorée de panneaux coupés comprenant essentiellement 2 personnages grambadant se faisant face, puis 2 animaux courant affrontés (fig. 44 b).

e) Fragment de bol, avec décor à métopes à figures peu reconnaissables.

1) Vase de forme parliculière (variante de la f. Hermet 23). Incomplet, couleur rouge mat. l.e bord est muni d'un bec d'écoulement semi-cylindrique et à $4,5 \mathrm{~cm}$. de celui-ci d'une anse formée par un ruban d'argile appliqué horizontalement à extrémités repliées. I.e décor, bien en relief, est réparti en 2 zones superposées séparées par un cordon à éléments trifoliés entre 2 lignes tremblées. La partie supérieure représente, sous un couronnement d'oves à orle festonné, une alternance de décor animal (bélier courant à droile) ${ }^{59}$ et ornemental (sautoir simple timbré d'une étoile aux angles

(58) (oswat.), op. cil., pl. XLVII, no 961. Uенмeт, I.a Graufesenque, pl. 23, no 268.

(59) Oswali), pl. I.X.Ti, no 1855 ; HeRмET, pl. 27, n$^{\circ} 32$. 

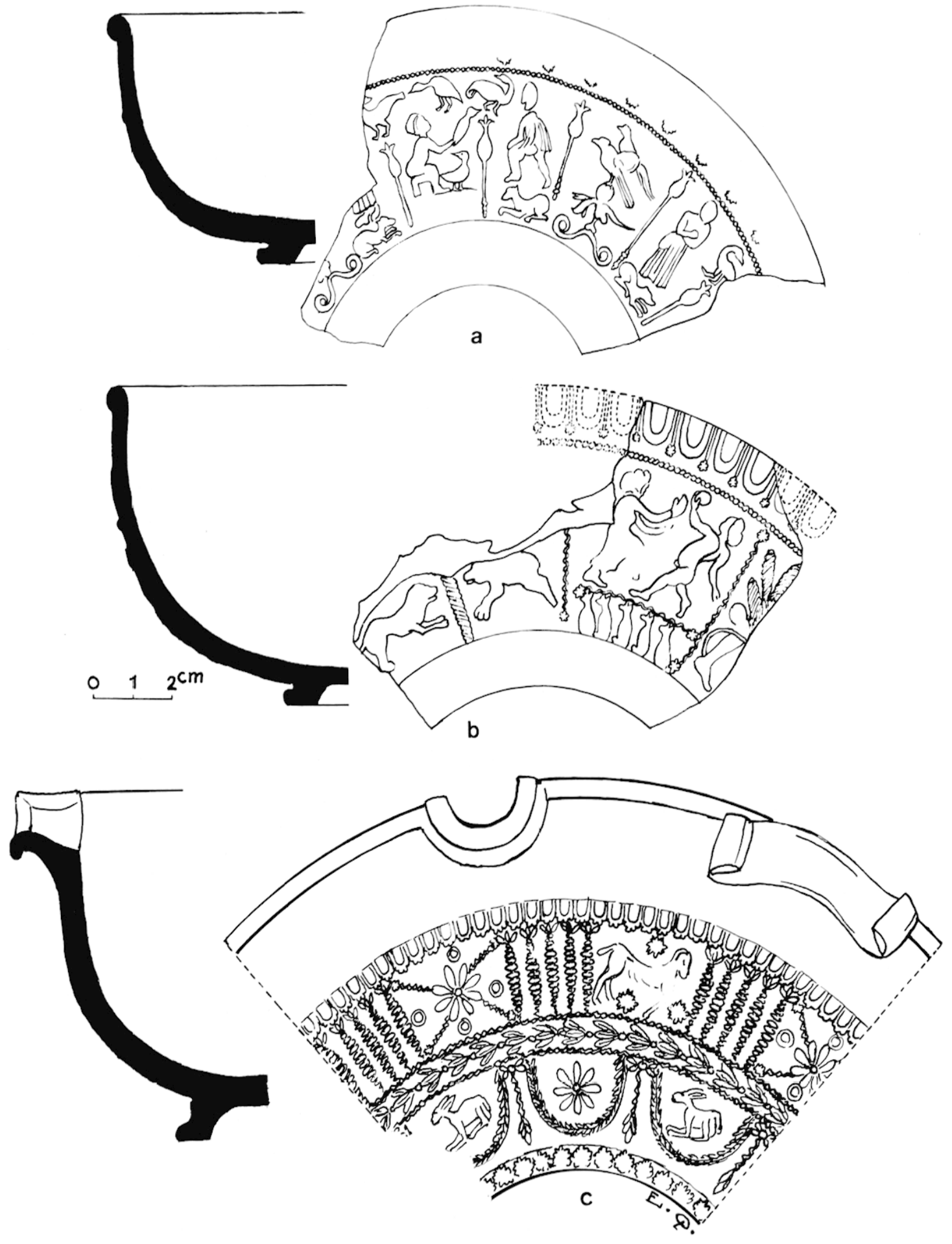

44. Puits $n^{\circ} 4$. V'ases siyillés ornes. 


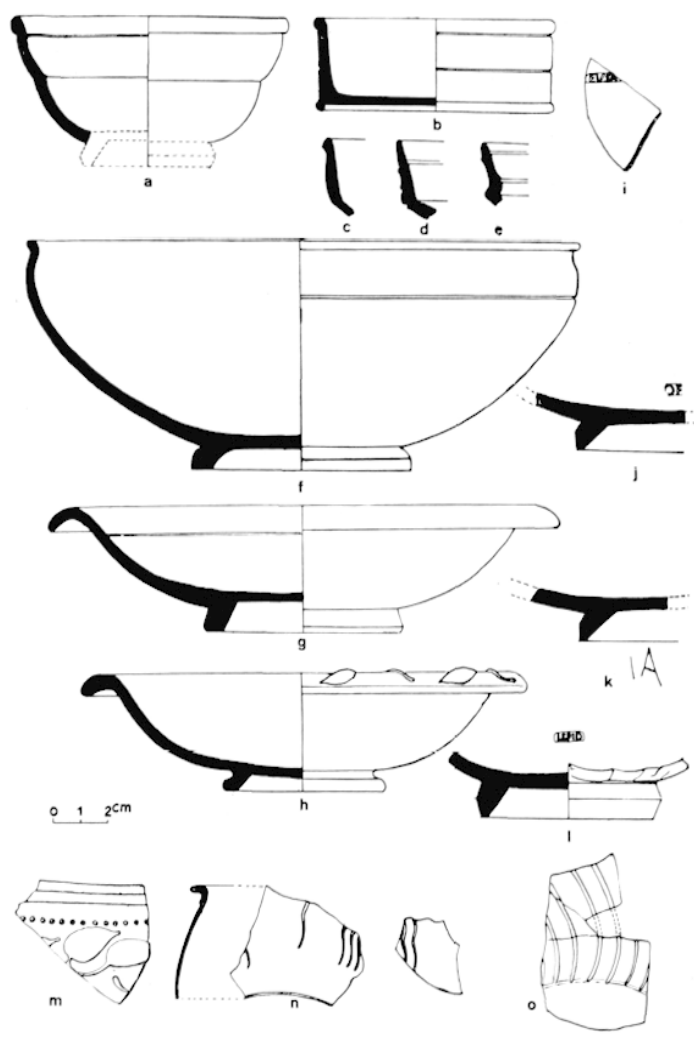

15. Puits no 1. Cimamique sigillie mnie et rases i parois minces.
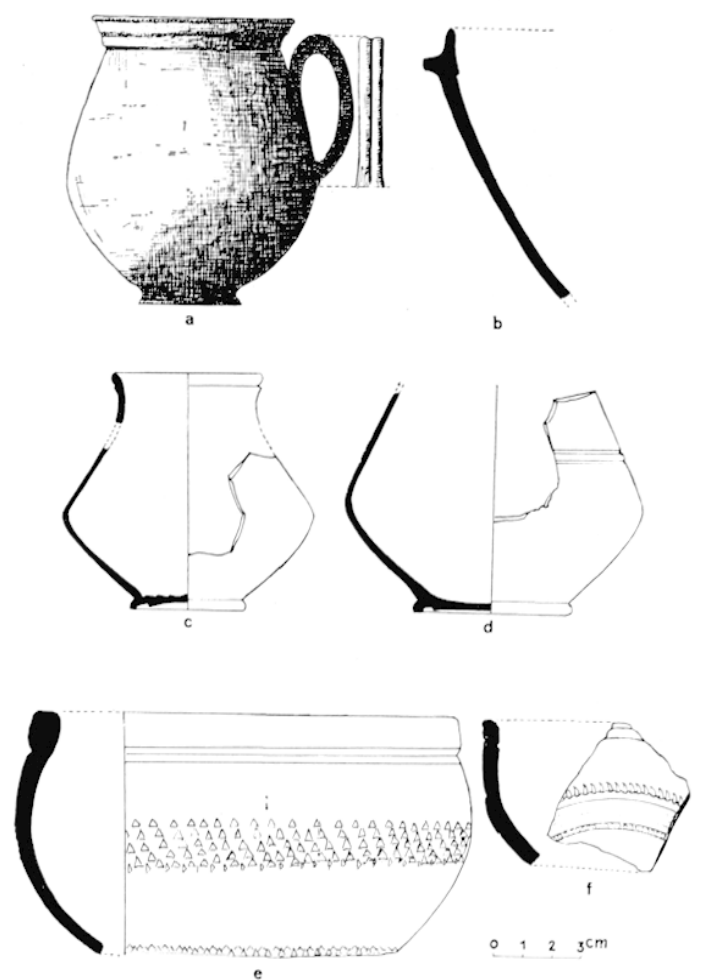

46. Puits no 4. Sigillie claire.

et d'un annelet aux 4 quartiers avec rosace centrale à 8 pétales.). Entre chaque type de décor se place une palissade de $\bar{y}$ tortillons, surmontés chacun d'un fleuron. A la partie inférieure, série de festons en demi-cercle, au centre desquels se trouvent alternativement, une rosace à 8 pétales, et un liìre au repos tourné à gauche ${ }^{60}$. Au soubassement un cordon étoilé circulaire (la Graufesenque, ép. Néron-Vespasien) (fig. $44 \mathrm{c}$ ).

g) Écuelle a bord décoré a la barbotine sur le dessus, de feuilles cordiformes a long pédoncule (H. : $3,1 \mathrm{~cm}$. I). : $16,3 \mathrm{~cm}$.). (fig. 4i) h).

$2^{o}$ Vases unis. a) Fragments importants : d'un bol à 2 bourrelets et une lèvre arrondie (f. Drag. $27^{61}$ (fig. 45) a) ép. Néron-Vespasien, d'un vase sessile, forme petite (Drag. 22) époque flavienne ${ }^{62}$ (fig. $45 \mathrm{~b}$ ), d'une écuelle à bords rentrants (f. Hermet 24) (fig. 45 f), d'une écuelle lisse (f. Drag. 36) (fig. $45 \mathrm{~g}$ ), de bords d'assiettes (f. Drag. 15/17) (fig. $45 \mathrm{c} \mathrm{d} \mathrm{e}$ ).

$3^{\circ}$ Marques de potiers. Fond de bol estampillé L E P I D (VS') (fig. 45 i) la Graufesenque, période Tibère-Néron ${ }^{63}, 2$ fragments de fonds avec marque incomplète : sur l'un EVsT: (T et $A$ liés). (peut-être EVsT:IDIVs, de Rheinzabern, période Hadrien-.Intonin) ${ }^{64}$ (fig. $45 \mathrm{i}$ ), sur l'autre $0 \mathrm{~F} \mathrm{(....}$ (fig. $45 \mathrm{j}$ ).

$4^{0}$ Graflite : I.l à la partie inférieure d'un pied de vase, à la pointe, après cuisson (fig. 45 k).

(60) Oswat.d, pl. LXXX, no 2095 ; HeRMI:T, pl. 26, no 66.

(61) Oswat.b, pl. XLIX, no 11 .

(62) Id., ibid., pl. L, no 10.

(63) Id., ibid., p. 161.

(64) Id., ibid., p. 117. 
E. Sigillée claire : $1^{\circ}$ Vase ovoïde tronqué, en terre jaune clair à couverte brun clair, muni d'une anse verticale à rainure médiane, court bourrelet au col, pied petit circulaire plat, bord à lèvre arrondie (1 ex. reconstitué aux $2 / 3$ et fragments de 5 vases de mème type) forme $26 \mathrm{~B}^{65}$ (fig. 46 a).

$2^{o}$ Petit vase à panse carénée (argile fine à glaçure orangée, extérieure seulement), (incomplet) (fig. $46 \mathrm{c}$ ).

$3^{\circ}$ Vase semblable au précédent, avec 2 sillons horizontaux à la base du col (fig. $46 \mathrm{~d}$ ).

$4^{0}$ Fragment de panse de grand bol décoré de petits triangles (type $\mathrm{B}$ forme $8 \mathrm{~b}^{66}$ (fig. $46 \mathrm{e}$ ).

50 Fragment de panse de bol de type $\Lambda$ (forme $I$ b) avec 2 rangées de stries simples ${ }^{67}$ (fig. $46 \mathrm{f}$ ).

$6^{0}$ Fragment de jatte à glaçure rose clair, bord a collerette horizontale (type $\mathrm{V}$ var. de la forme $38)^{68}$ (fig. $46 \mathrm{~b}$ ).

F. Vases à parois minces : $1^{\circ}$ Fragments de coupe i décor de feuilles d'eau sous une ligne ponctuée (fig. $45 \mathrm{~m}$ ) caractéristique de l'époque flavienne ${ }^{69}$.

$2^{\circ}$ Fragments, d'un petit vase à rebord horizontal, en argile grise très homogène, recouvert extérieurement d'un beau vernis noir glacé brillant, décoré à la barbotine de lignes serpentiformes verticales sur la panse (fig. $45 \mathrm{n}$ ).

$3^{\circ}$ Fragments d'un petit vase semblable au précćdent mais à glaçure marron avec lignes courbes continues, verticales (fig. 450 ).

G. Poterie grise fine : petite urne en terre cuite grisàtre ì engobe plus foncé ; panse globuleuse et pied étroit (fig. $47 \mathrm{~g}$ ).

II. Céramique commune. $A$. Urnes. Complètement diflérentes de celles du puits $\mathrm{n}^{0} 7$, ce sont les formes de l'époque des Flaviens-Intonins qui dominent. On peut en distinguer :3 lypes:

$1^{\text {er }}$ lype: olla en argile fine, dure, de couleur gris clair. La paroi externe porte les traces du tour. souvent accentuées près du col où elles dessinent des raies concentriques, alternant avec des zones lisses. Le fond est étroit et plat, quelquefois un peu concave en dessous. La panse est galbée, le col court et le hord en bourrelet arrondi : un exemplaire entier $($ H. : $12 \mathrm{~cm}$. D. $0 .: 12 \mathrm{~cm}$. D. M. : $15 \mathrm{~cm}$. D. $13 .:$ : $6 \mathrm{~cm}$.) (fig. 47 a), 2 autres plus petits $(10 \mathrm{~cm}$. et $7 \mathrm{~cm}$. de haut) ainsi que des fragments (surtout des bords et fonds) d'au moins 7 récipients semblables.

20 lype: olla de facture plus ordinaire et de taille un peu plus grande, en argile marron à petils grains de calcaire inclus dans la pàte. Les parois sont minces, leur surface externe est rougeàtre ou noirâtre avec zones lisses, les traces du tour sont particulièrement visibles à l'intérieur. La panse est surhaussée, à hase étroite, l'ouverture large avec rebord arrondi sur le dessus. Un de ces récipients a été reconstitué (fig. $47 \mathrm{~b}) . \mathrm{H} .: 17,4 \mathrm{~cm}$. D. $0 .: 17,2 \cdot \mathrm{cm} \mathrm{D}$. B. : $7 \mathrm{~cm} .^{70} .10$ sont incomplets; sur un col on remarque un signe gravé en forme de petit rameau (fig. $47 \mathrm{e}$ ).

3e type: olla en terre grise, à col concave avec épaulement très marqué. Panse galbée terminée par un pied bas débordant (fig. $47 \mathrm{c}$ ).

B. Bassine de forme tronconique renversée (type du puits $n^{0} 7$ ). Quelques fragments de la partie supérieure ornée des incisions obliques caractéristiques.

(. Jattes ou terrines. Récipients bas, à bord plus ou moins déversé, lond plat, large. 1 exemplaire entier (fig. $47 \mathrm{~d}$ ), 5) autres, incomplets, de profils variés (fig. $47 \mathrm{~d}^{\prime}$ ).

(65) X. Lamboglas, Nuove osservazioni sulla "lerra sigillala chiara", op. cil., p.1312, fig. 26.

(66) Id. ibid., p. 307, fig. 8 b.

(67) Id. ibid., p. 262, fig. ë b.

(68) Id. ibid., p. 311.

(69) X. Lamboril.re, Gli scavi di Albinlimilium, p. 125, fig. 27 à 30.

(70) Ce type d'urne est fréquent dans les tombes à incinération de la fin du a er s. ap. J.-C., notamment à Sannes Ise) : A. De moc t.Ix, Recherches archéologiques dans la région d'Apl, dans Gallia, XVI, 1958, p. 225, fig. 33. 


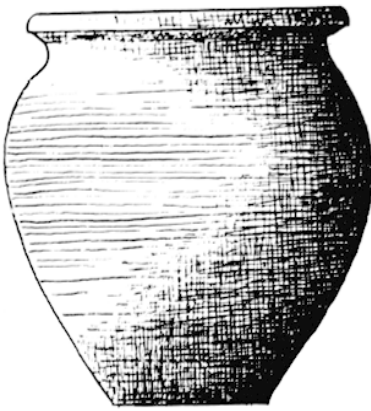

a

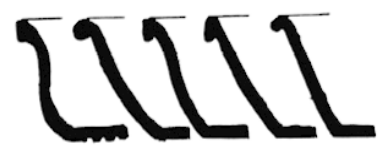

d'

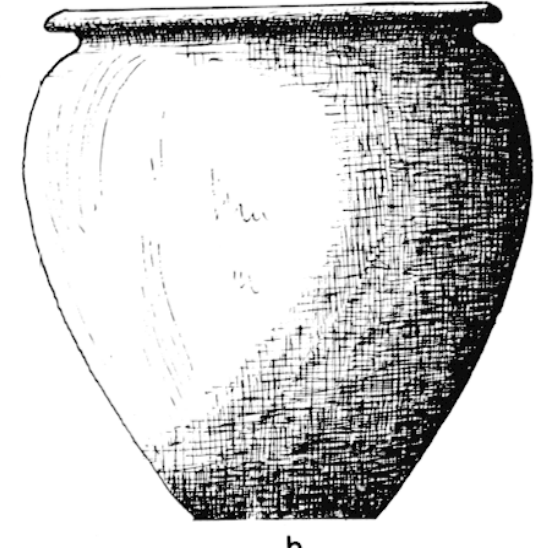

b

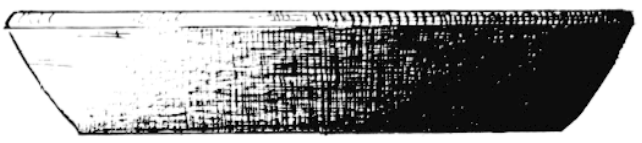

d

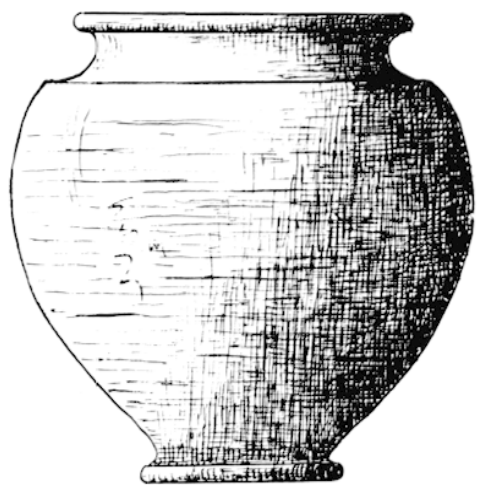

c

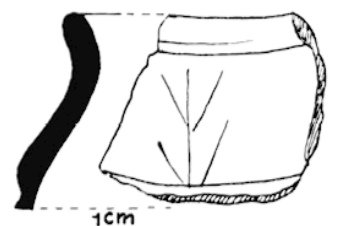

e

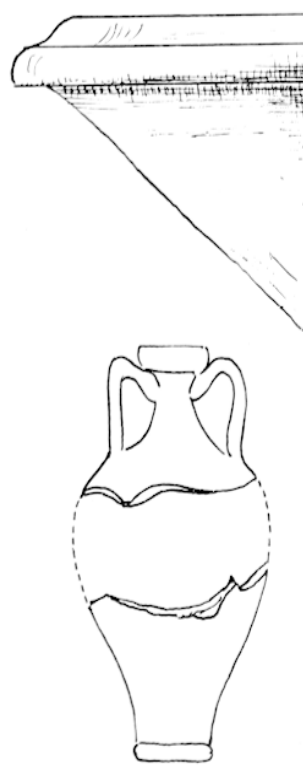

$\mathrm{h}$ $f$

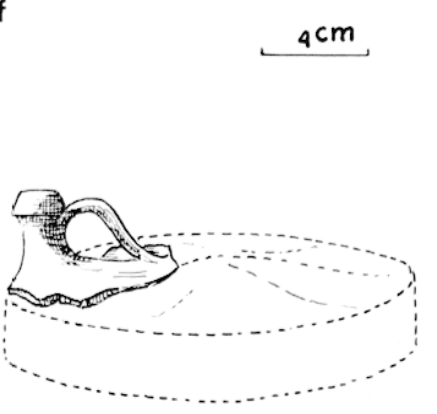

i
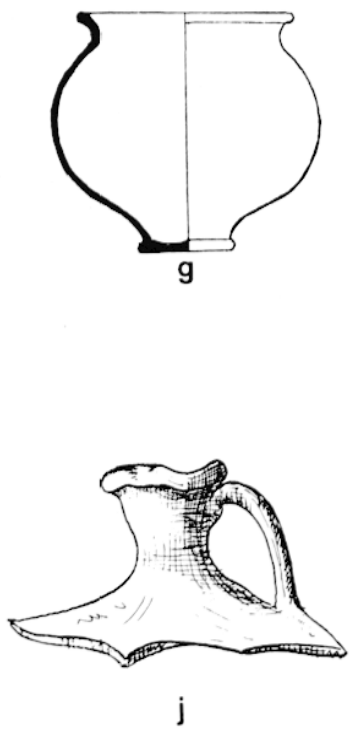

47. Puits no 4 . Récipients en terre commune.

III. Polerie jaune. Mortier à bec et collerette lisse (fig. $47 \mathrm{f}$ ) et 2 autres incomplets. Vase à liquide, sorte d'amphorisque à 2 anses coudées opposées et fond plat (fig. $47 \mathrm{~h}$ ). Vase à liquide à une seule anse coudée et panse large (3 ragments), bec de cruche, type à anse torsadée, bord d'urne ordinaire, fragrments d'amphores à fond conique.

IV. Polerie gris fer. Vase à liquide à bec tribolé (partie supérieure) (fig. $47 \mathrm{j}$ ) et fragment d'une 
gourde horizontale, circulaire, plate, munie d'un goulot se rattachant au dos du récipient par une anse oblique à sillon médian (fig. $47 \mathrm{i})^{\mathrm{a}}$.

V. Poterie à vernis plombière. Fragment de panse de vase, vert foncé, brillant à l'extérieur, jaunâtre à l'intérieur ${ }^{72}$.

VI. Lampes en terre cuile. 8 lampes incompletes et une vingtaine de fragments de plusieurs autres.

A. Type de la Vallée du Pò (forme Dressel 5. Var. A) : fragment d'une grande lampe d'environ $16 \mathrm{~cm}$. de longueur, en argile rouge brique, sans engobe. Il manque tout le réservoir et une partie du dessus, au centre duquel se trouve un grand trou d'alimentation. L'orifice de la mèche $(2 \mathrm{~cm}$ de diamètre) est noirci. Lne gouttière longitudinale joint le bord de la cuvette, avec lequel elle ne communique pas, et le milieu du bec. $\perp$ noter 2 petits trous supplémentaires dans le même alignement, au bas de la gouttière et du dessus (fig. 48 a).

B. Type à volutes et bec rond (forme Dressel 11 type B). 1. Lampe en argile blanchâtre à couverte orangée. Le dessus, qui devait être orné, est très incomplet et porte 3 petits sillons circulaires concentriques (H. : $2,5 \mathrm{~cm}$. L. : $11 \mathrm{~cm}, 5.1: 8,2 \mathrm{~cm}$.) (fig. $48 \mathrm{~b}$ ).

2. Lampe en argile jaunàtre à couverte rose clair. Sur le dessus, à 2 sillons circulaires : 2 dauphins affrontés, la tête en bas, de chaque còté du trou central (H. : $2,9 \mathrm{~cm}$. I. : $8,2 \mathrm{~cm}$. $1 .: 6,7 \mathrm{~cm}^{73}$ (fig. $48 \mathrm{c})$.

3. Lampe à couverte brune. Le dessus est orné d'une sphynge accroupie de face $\mathrm{e}^{74}$; sur le fond : marque du fabricant L. HOS. CRI ${ }^{75}$ (fig. $48 \mathrm{~d}$ ).

4. Fragments de 4 autres lampes de même genre.

(.. Type a volutes en virgule et bec rond (type $C$ de de Brun et (iagnière) ${ }^{76}$. Lampe en argile claire à couverte marron, 1 sillon circulaire, lettre $\mathrm{N}$ sous le réservoir (fig. $48 \mathrm{e}$ ).

D. Type a bec rond sans volutes (f. Dressel 21, sans anse). 1. Lampe ì sillon circulaire du couvercle interrompu au départ du bec, où il se coude en 2 petits segments parallèles limitant un court canal, coupé par une incision rectiligne à 2 points creux à chaque bout". L'ornementation consiste en 2 palmes, à nervures nombreuses, de part el d'autre du trou d'aération ${ }^{78}$ (fig. $48 \mathrm{f}$ ).

2. Lutre lampe semblable à décor plus stylisé (fig. $48 \mathrm{~g}$ ).

E. Divers. Partie postérieure de lampe en argile orangée à engobe de teinte plus foncée, qui rappelle celle de la sigillée claire, type $A$ forme I b décrit plus haut, munie d'une anse verticale perforée située sur la tranche (fig. $48 \mathrm{~h}$ ) ; 2 fragments : l'un avec rosace, l'autre, une jambe humaine (fig. $48 \mathrm{ij}$ ).

(71) Les sépultures du Gard ont donné, parmi les vases constituant le mobilier funéraire, un récipient semblable: S. Gacixil:re, Sëpultures gallo-romaines de la Seignourelle à Villeneuve-les-Avignon (Gard), extr. des Cahiers d'IIist. el d'Arch. de Nimes, 1933, p. 3, pl. I, nº 5. Les couches supérieures d'une grotte préhistorique de ce même département en ont fourni des fragments: S. Gagivi:Re et J. Gravier, Les niveaux hisioriques de la grolle du lierre à Saint-Geniès-deComolas (Gard), extr. de Provence hislorique, no 48, 1962, p. 186, fig. 4 et 4 bis.

(72) Quelques tessons de cette poterie, toujours rare, ont été trouvés dans un dépotoir près de Glanum : P. de Brcx, Noles sur la céramique anlique à Saint-Rèmy-de-Provence, IJ c partie, Succession des lypes, dans Rhodania, 1932, p. 9 du tirage à part. $\mathrm{n}^{\circ} 155$.

(73) P. de Brux et S. Gagivière, Les lampes anliques du .Musée Calvel d'Avignon, Carpentras, 1937, p. 38, pl. X,

(74) Id. ibid., p. 25, no 48 : lampe avec le même décor et la même marque, pl. IV, no 47.

(75) Sur l'atelier de ce potier: R. BaILL.y, op. cil., p. 101 à 106.

(76) P. de Brun et S. Gagivitre, op. cit., p. 14, pl. I, type C.

(77) M. Labrolsse, Lampes romaines de Montans aux Musees de Toulouse el d'Albi, dans Mém. de la Soc. arch. du Midi de la France, XXVIII, 1962, p. 22, fig.l5, forme du $11^{\mathrm{e}}$ siècle ap. J.-(...

(78) P. de Brun et S. Gaginitire, op. cil., p. $41, \mathrm{n}^{\circ} 76$. 

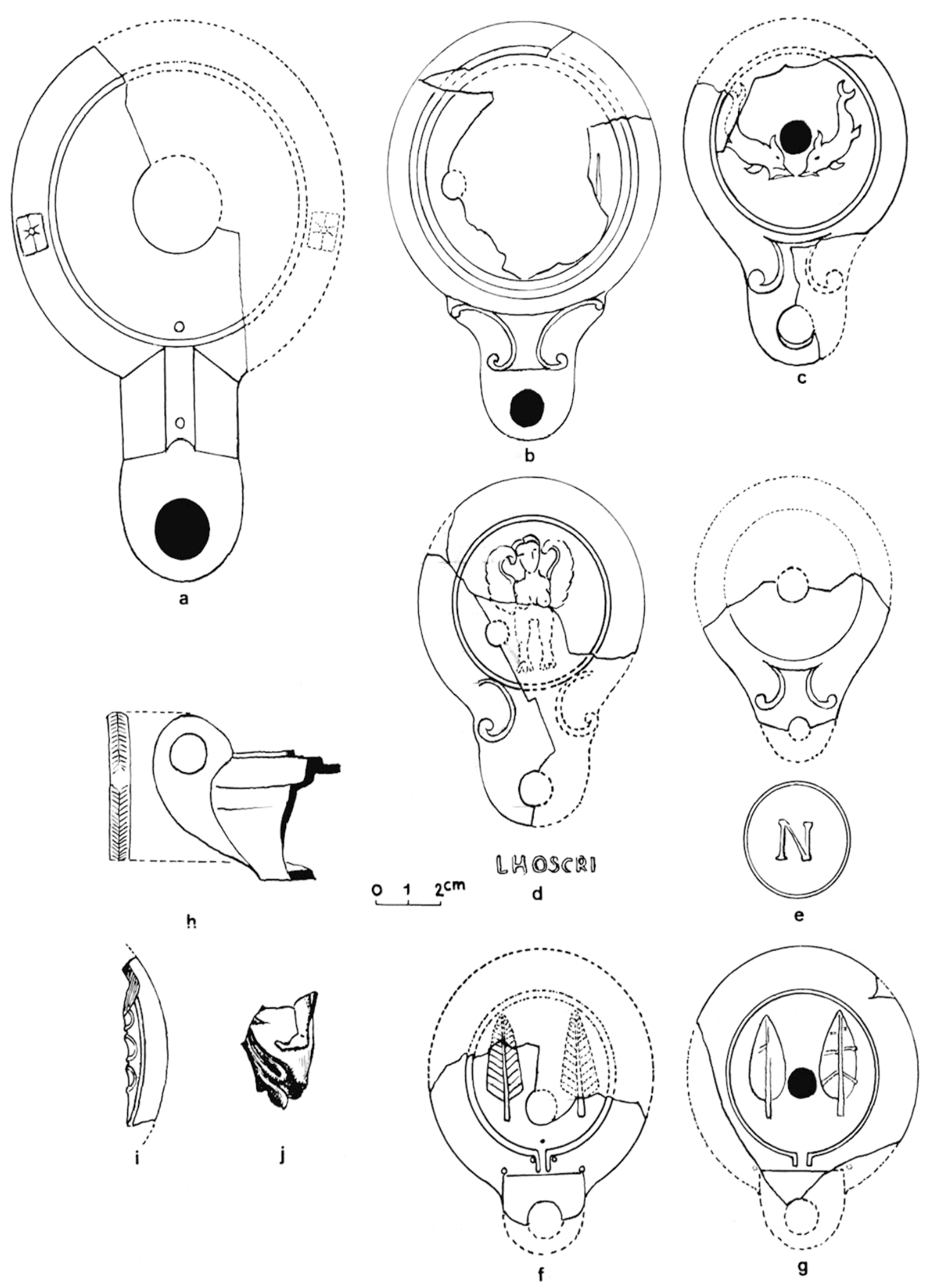

48. Puits $n^{0} 4$. Lampes en terre cuite. 


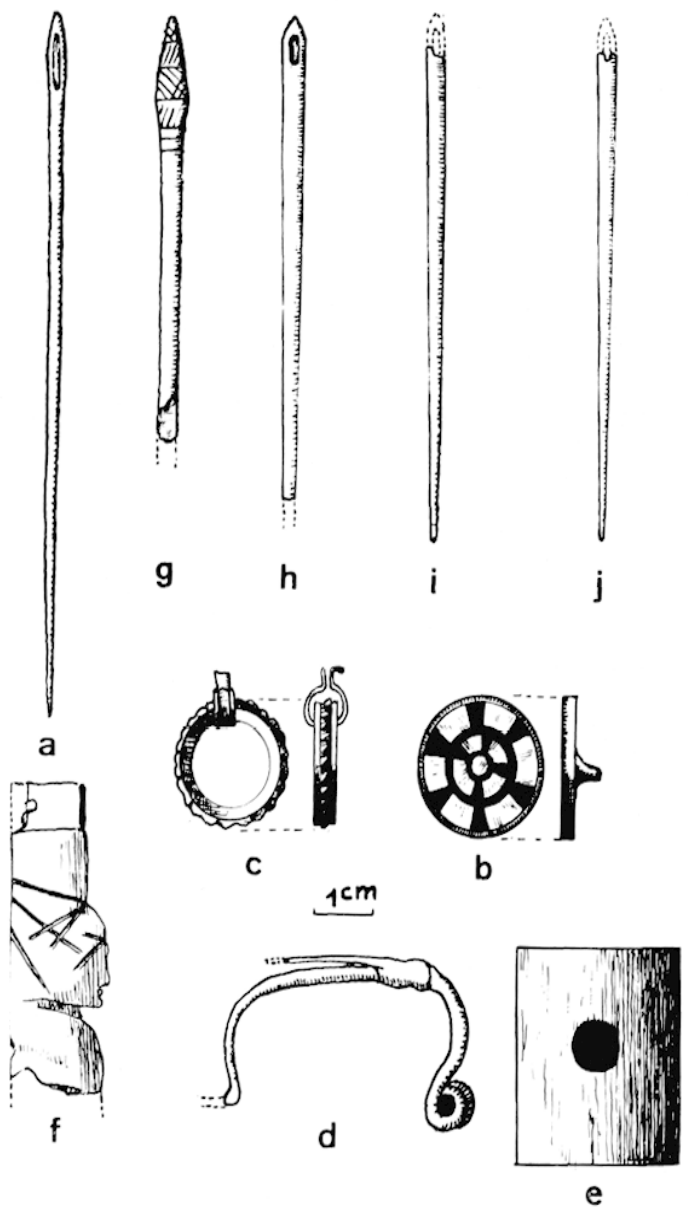

49. Puits $n^{\circ}$.1. Ol,jets en bronze et en os.
VII. Verrerie. Deux fragments de gobelets de teinte claire, a fond concave el parois épaisses (types de la fosse $n^{0} 3$ ).

VIII. Bronze. 1. Aiguille a chas ovale allonge (L. : $11 \mathrm{~cm})(\mathrm{fig} .49 \mathrm{a})$.

2. Fibule de la Tine $11^{79}$ incomplète comprenant un arc coudé de section circulaire dont la tête est terminée par un ressort brisé a la 2 e spire. Le pied montre le départ du porte-agrafe qui était relié au dos de l'arc par un appendice dont il reste encore la bague de raccord. (fig. $49 \mathrm{~d}$ ).

3. Inneau mince, de $1,8 \mathrm{~cm}$. de diametre. à crête sinueuse périphérique avec fragment de chainet te entourant encore l'anneau (fig. $49 \mathrm{c}$ ).

4. Petit disque en forme de bouton plat piqueté tout autour, incrusté sur une face, d'émail vert et rouge avec zones disposées en rayons autour de 2 cercles concentriques. Le revers, uni, porte au centre le départ d'une lige coudée de seclion quadrangulaire qui devait se prolonger comme une épingle (I). : $2 \mathrm{~cm}$. Ep. : $0.2 \mathrm{~cm}$.) (fig. 49 b).

5. Fragment de plaque de forme arquée.

IX. Os. I. 3 aiguilles ou passe-lacets 80 a chas (I. : 7.5) ('m.) (lig. 49 h i j).

2. Epingle a coilfure. à libe biconique. orné de stries obliques disposées tout autour sur 4 \%ones horizontales superposées (figr. 49 gr).

3. Fragment de plaquette polie el enlaillée sur une face. Lne de ses extrémilés amincie, porte un Irou de rivetage el ressemble toul a fait i la parlie du manche des coul eaux qui s'engage dans la virole. Le centre parait avoir éte façonné pour obtenir le profil d'un buste humain (fig. $49 \mathrm{f})$ (L. : 1,5) ('m.).

4. Rouleau de charnière. conslitué par un trongon d'os long de bovidé en forme de mancluon de crlindre soigneusement poli (11.: :3,3 cm. I). :3,9 cm.). Cin trou régulier parfore la paroi de l'objel a érale distance des 2 bases (H. : :3,3 (cm. D. : :3,9 cm.) (fig. $49 \mathrm{e}$ ).

5. Fragments de cote et d'omoplate de boruf qui portent les traces des coups d'un outil tranchant.

X. Objels divers. 20 clous très oxydés ainsi que des morceaux informes de mème mélal ; plaque de plomb tordue ; rondelles en mollasse de forme légirement tronconique (H.:3 (cm. I). : 11, a) (m.) : moitié de couronne en poterie.

XI. Faune. Nombreux os de breuf et mouton, dents de sanglier, coquilles marines (huiltre, moule, pecten et un pelit cardium) et terrestres (hélix et bulimes).

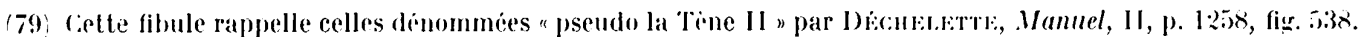

(80) 1 in objet semblable est fịnuré sous le nom de passe-lacet dans le llel. des. Inl. Cirecques el romaines de Jaremberg et saglio. p. (il, fie. s8. 


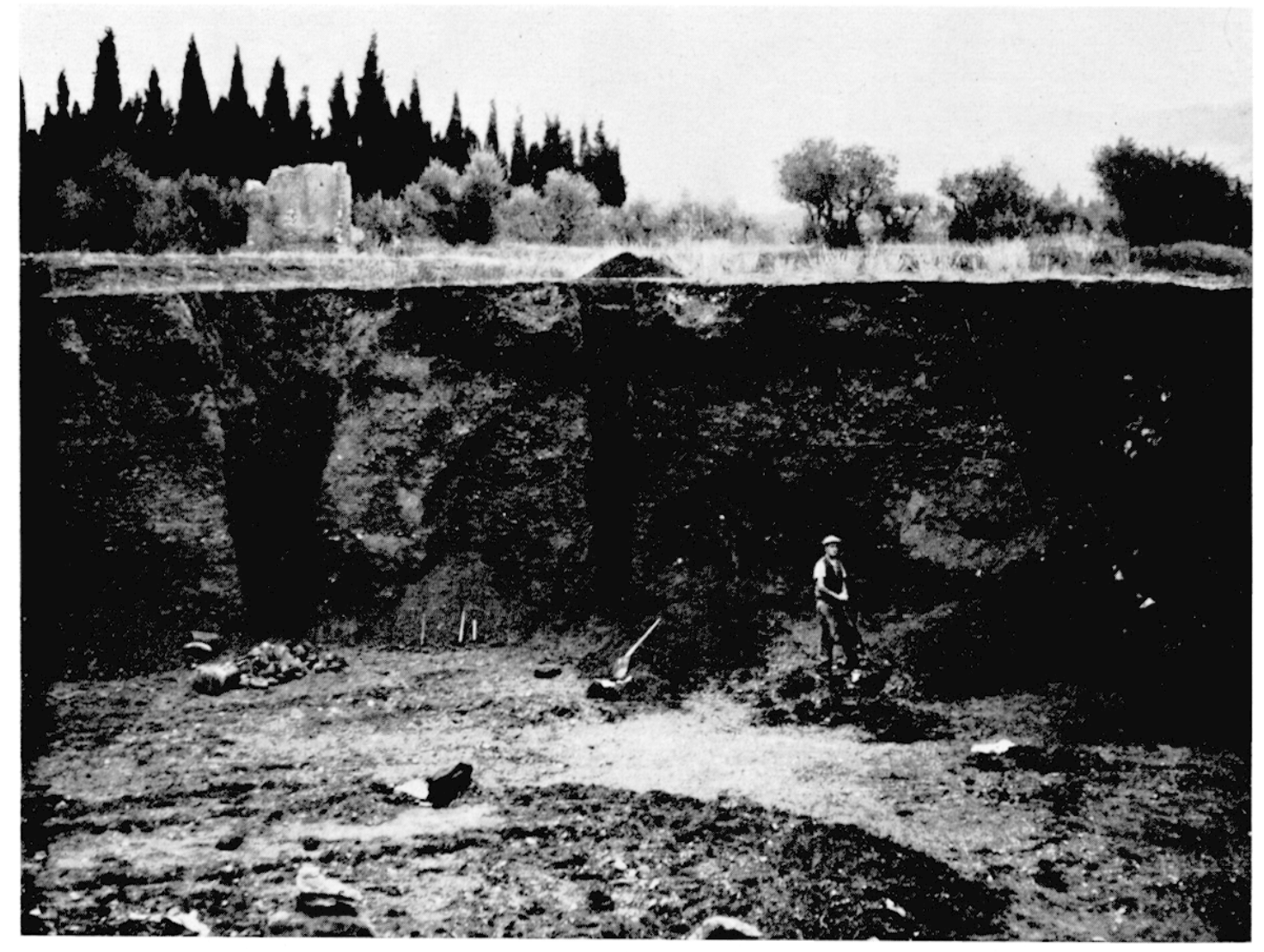

50. Carriere Thiebold. De gauche a droite : puits $n^{\circ} 4$, fosse $n^{\circ} 1$, puils $n^{\circ} 5$. On remarque l'évasement du puits $n^{\circ} 4$, l'étroitesse et l'arrèt en profondeur de la fosse $n^{0} 1$ et la moitio de la margelle coincre dans le puits $n^{\circ} 5$.

Le comblement final de ce puits s'est effectué plus tard que les autres, avec de la céramique el des lampes du ite s. ap. J.-l:.

PIITS $x^{0} \bar{\jmath}$

Carrière Thiébold (septembre 1941). Ce puits avait la c'urieuse particularité de n'être comblé que dans sa moitié supérieure. Lors de sa découverte, la colonne de terre qui le remplissait apparut, arrêtée en profondeur par une moitié de margelle qui étant tombée dans le puits, s'était coincée en travers au bout d'une chute de 6 mètres (fig. 50). Au-dessous, la cavité cylindrique verticale, d'un diamètre de $1,50 \mathrm{~m}$. était vide sur une hauteur de $8 \mathrm{~m}$. et laissait voir au fond la nappe d'eau. Le haut de ce puits contenait des tessons de poterie au milieu de décombres : moellons, plaques de mortier avec enduits muraux vert uni, tuiles à rebord, déchets de cuisine.

I. Poterie fine. A. Phocéen : fragment de pied profond de coupe en argile grise, tendre avec traces de couverte foncée, et 2 petits tessons (imitation régionale).

B. Campanien : 8 petits fragments (bord, panse. fond) d'écuelles, en Camp. $A$. 


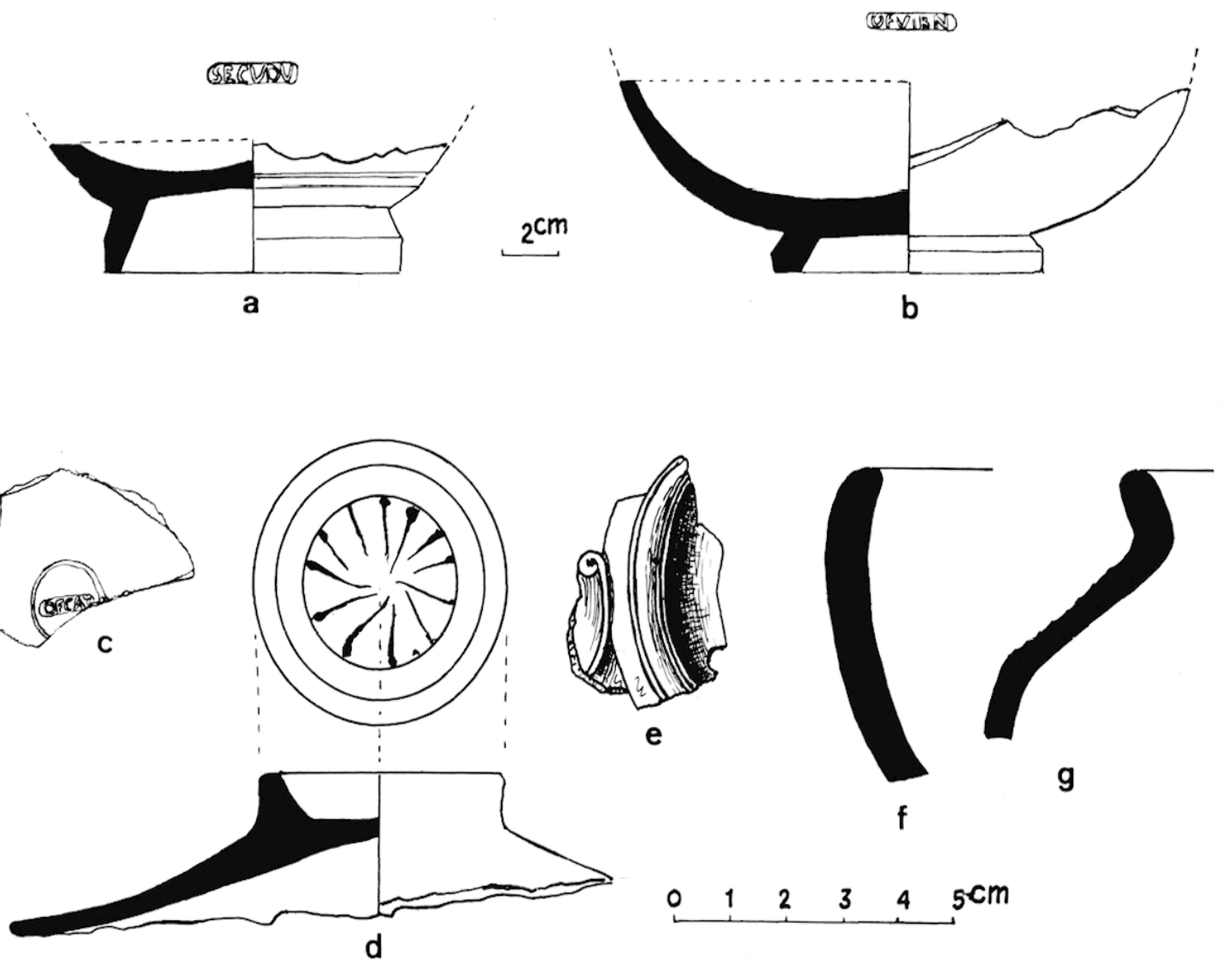

51. Puits $n^{\circ} 5$. Poterie sigillée, commune et fragment de lampe.

c. Imitation de Campanien : 2 tessons à vernis marron foncé, et :3 autres, à vernis très clair.

D. Céramique arénacée à texture grenue, enduite d'un engobe rouge épais couleur lie de vin a l'intérieur seulement : fragment de bord concave de jatte de grandes dimensions (fig. 51 f).

E. Sigillée: 1. Fond de bol tronconique estampillé SECVNDV (S) (V, $\mathrm{N}$ et $\mathrm{D}$ liés) (la Graufesenque, période Claude-Vespasien) ${ }^{\mathbf{8 1}}$ (fig. $51 \mathrm{a}$ ); fond de coupe avec marque peu lisible, peut ètre OFVIBN (VIBIVS, potier de Montans période Claude-Vespasien ${ }^{82}$ (fig. $51 \mathrm{~b}$ ) ; Fragment de fond de vase avec marque empâtée, sans doute OFC.INT (C.INTUS) (la Graufesenque, période Tibere-(ilaude) $)^{83}$ (fig. $51 \mathrm{c}$ ).

2. 7 petits fragments appartenant aux formes Drag. 18, 23, 24, 25 .

11. Lolerie commune. 17 fragments d'urnes à rebord horizontal (5 récipients différents) of, 2 fragments en terre noire à petites paillettes de mica dont 1 bord d'olla à col coudé (fig. ந̂l g).

2 couvercles incomplets d'olla, dont 1 pied avec lignes rayonnantes gravées avant cuisson, extérieurement (fig. 5l d).

Fragment de grande bassine à encoches obliques sur le bord (type du puits no 7 ).

(81) (Oswal.), op. cil., p. 288 (in fine).

(82) Ill., ibid., p. 333.

(83) Il. ibid., p. 58. 
III. Lampe. Fragment d'une lampe en terre jaune à vernis brun brillant à 3 sillons sur le dessus et volute saillante latérale pres du bord (fig. $51 \mathrm{e}$ ).

IV. Margelle. La moitié de margelle placée en travers du puits était un bloc de mollasse blanche de $1,40 \mathrm{~m}$. de long, $0,70 \mathrm{~m}$. de large et $0,35 \mathrm{~m}$. de haut, largement échancré sur un còté, qui devait coiffer avec une moitié semblable l'orifice du puits et constituer ainsi une margelle carrée à 2 éléments juxtaposés. sur le dessus on remarque 2 encoches profondes, points d'appui de 2 des 3 montants en bois, croisés et attachés plus haut pour le soutien de la poulie (fig. 3, reconstitution).

V. Divers: moitié inférieure d'amphore romaine pansue à pied conique, de fond plat de flacon en verre clair, et une vingtaine de grands clous en fer.

VI. Faune: Gros os et mâchoire de buruf, mouton, porc et sanglier.

Le cas fortuit du comblement incomplet de ce puits, dû au "bouchon " formé par le bloc de margelle tombé de la surface, sur lequel est venu s'accumuler une couche archéologique allant du ${ }_{1}^{\text {er }}$ s. av. J.-C. au ${ }_{11}$ e après, illustre bien sa double utilisation successive, qui est du reste, celle de tous les autres : puits à eau, ensuite dépotoir. L'époque de ce changement se situerait donc, pour celui-ci, vers le milieu de la Tène III.

\section{PUITs $x^{\circ} 6$}

Carrière Thiébold (Mai 195う). Il avait $1 \mathrm{~m}$. de diamètre et a pu être fouillé sur une hauteur de $4 \mathrm{~m}$. 75. La terre de remplissage contenait à la partic supérieure, des fragments de tuiles plates à rebord (legulae) et de tuiles couvre-joints (imbrices) mêlées à de gros galets de la Durance et à des fragments de poterie.

I. Poterie fine. A. Protocampanien : 7 pctits fragments de coupelles à vernis noir très brillanl.

B. Campanien $A$. Une vingtaine de menus tessons appartenant à des bols ou coupes.

C. Campanien B : fragment de patère el de coupe.

I). Imitation régionale du Campanien. Une dizaine de fragments d'écuelles à vernis marron métallique (cf. puits $n^{0} 7$ ).

E. Campanien tardif : fragment de pied creux à vernis bistre avec 2 belles empreintes digitales; fragment d'écuelle en argile jaune clair à vernis marron brillant. $n^{0}$ 7.).

F. Campanien C. : 4 fragments d'un grand plateau à 2 zones hachurées concentriques (cf. puits

G. Imitation de Campanien $C:$ fragments de coupe carénée et de bord de bol.

H. Pré-sigillée. 8 fragments d'une coupe à sillon près du bord (f. Ritt. 8 ).

I. Sigillée : a) Poterie arétine : fragment de fond de petit vase avec estampille en 2 lignes, mal imprimée ...VFI peut ètre RVFIO ${ }^{84}$ (fig. 52 a).

....ON

(84) Marque connue à Saint-Rèmy-de-Provence : II. Rol.LANd, Inscriplions anliques de Glanum, dans Gallia, II, 1944, p. 208, no 39. 

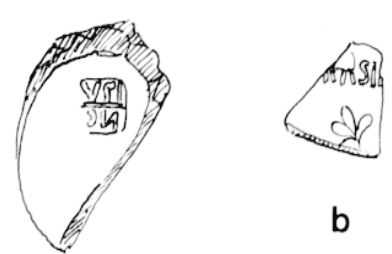

a
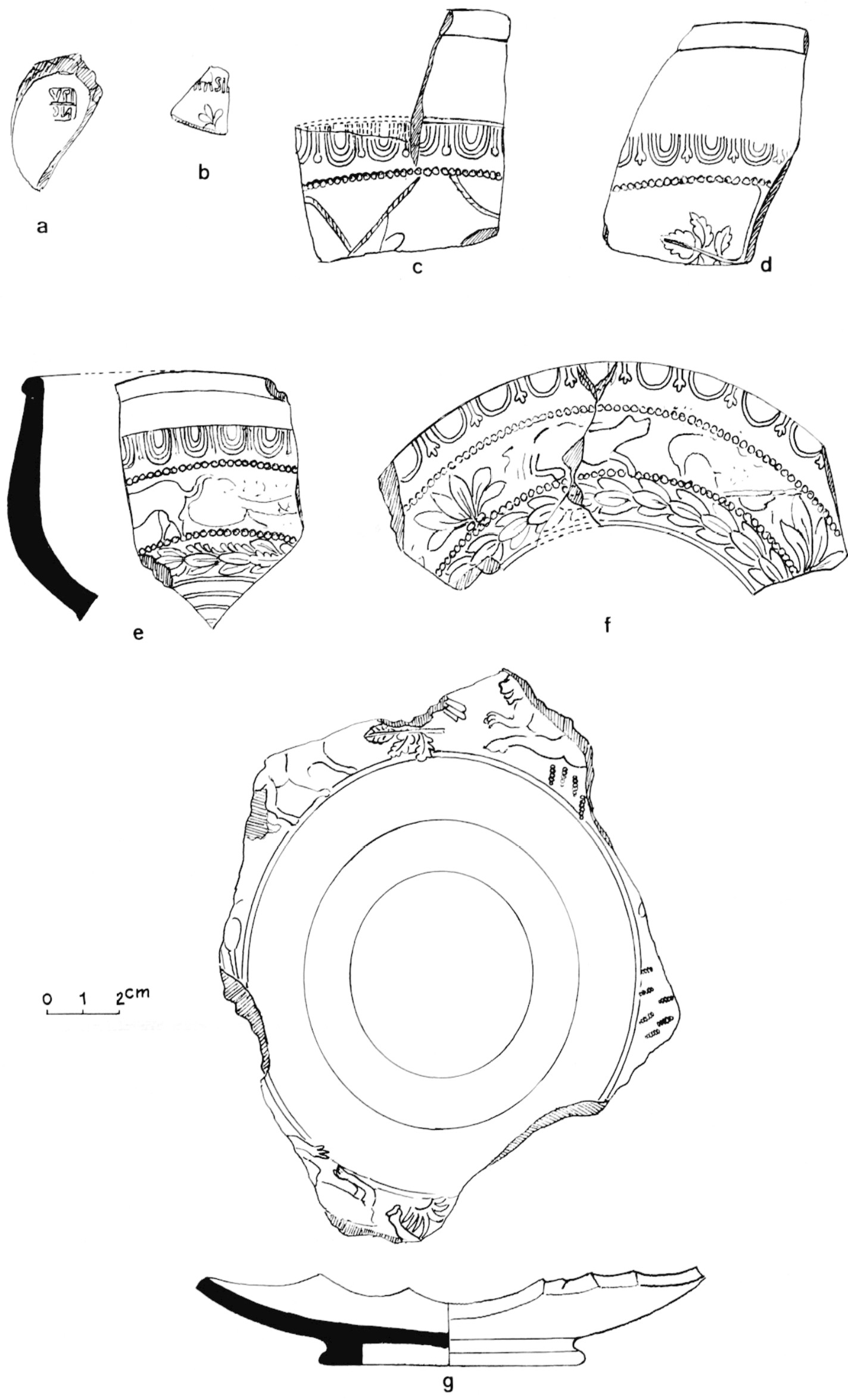

52. Puits no 6. Poterie sigillée. 
Fragment de paroi de bol orné d'une sorte de feuillage, avec marque intra-décorative (R.I) SI (.II $)^{85}$ (fig. $52 \mathrm{~b}$ ). 26 petits tessons prarmi lesquels on peut distinguer 1 fragment orné d'un tortillon, plusieurs bords de f. Haltern 7 et Ritt. i), el de tasse à rayures parallèles près de l'ouverture (cf. puits no 7 ).

b) Fragments de 2 vases ornés f. 37, à motifs flous et mal moulés (atelier de Rheinzabern ?), trouvés dans la partie haute du puits. Le 1 er représente des sant nirs simples avec feuilles (fig. $52 \mathrm{c} \mathrm{d}$ ), le $2^{e}$, des animaux courant, séparés par un buisson à éléments divergents, le tout sur une guirlande trifoliée (fig. 52 e f). Lin 3 e vase. f. 37 , dont il reste seulement la base avait une ornementation figurée dont on ne voit que l'extrémité inférieure (fig. .52 $\mathrm{g}$ ).

J. Poterie a parois minces : 23 petits fragments parmi lesquels on peut reconnaitre le petit vase à décor de triangles, la pointe en bas, barbotinés (cf. puits $n^{0} 7$ ) et l'unguentarium à col allongé.

K. Céramique lustrée, non tournée : ( ; fragments de jattes à cótés obliques, peu élevées. de couleur orangée el 7 fragments de bords de coupes a pied marron foncé (4 récipients dillérents).

II. Polerie commune. 1. Lines : 160) Pragments parmi lesquels on reconnait l'olla de tradition hallstattienne à bord évasé. l'olla de la Tine à rebord horizontal, l'urne à panse surhaussec (type du puits $n^{0}$ 4) avec, en plus, une urne en argile marron terre de sienne aver petits grains de ralcaire, a panse ronde et bord droit.

B. Couvercles d'olla: 1 moitié de pied.

(. Bassine : 3 fragments de bords avec incisions ; 1 fragment de panse a anse pleine.

I. Plateau à pain : 1 fragment de fond épais.

III. Céramique gris fer. 1 fragment de vase a liquide, 1 fond, 2 fragments de bord et 1j de panses d'urnes.

IV. Polerie jaune. 1. Vase à liquide recons-

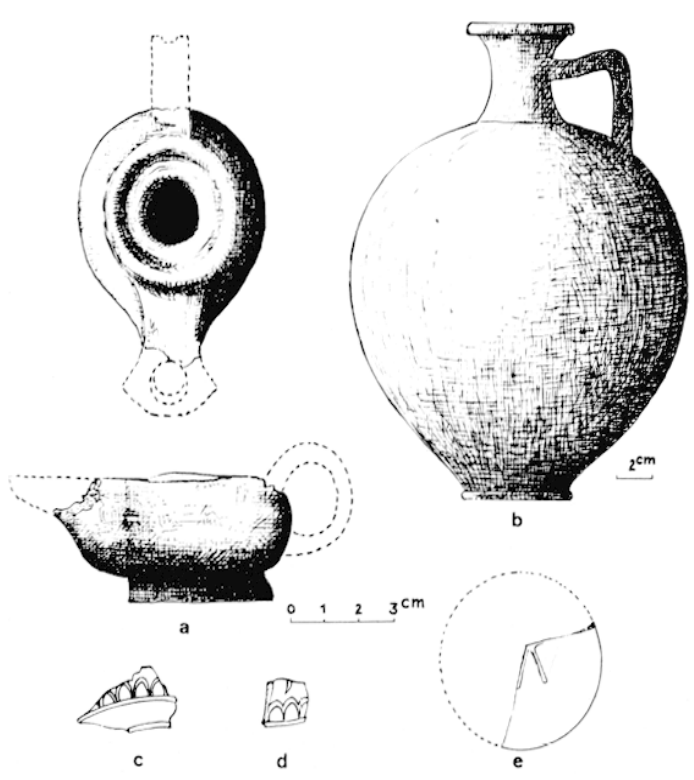

53. Puits no 6. Lampe campanienne, vase a liquide en terre jaune el fragments de lampes du I $^{\text {er }}$ siecle après .J.-C.. titué en entier, à panse sphérique, goulot court arqué et petite anse coudée, pied pelit évidé (H. : $26 \mathrm{~cm}$. DM. : $22 \mathrm{~cm}$.) (fig. $5.3 \mathrm{~b}$ ) et 158 tessons de vases à liquide (type à anse torsadée et formes diverses rencontrées dans le puits $\mathrm{n}^{\circ} 7$ ).

2. Fragment de mortier, goulot d'amphore à anses arrondies, couronne en terre cuite orangée pâle, un peu aplatie.

V. Lampes. 1. Lampe en poterie campanienne (le bec et l'anse manquent). Dessus en cuvette étroite avec grand trou d'alimentation central, pied circulaire creux avec trace digitale en dessous ${ }^{86}$ (H. : $3 \mathrm{~cm}, 5$. L. : $7 \mathrm{~cm}$ ) (fig. 533 a).

2. Une dizaine de petits fragrments dont 2 sont ornés d'une bordure d'oves (fig. 5.3 c d) et un fond avec lettre $V$ en relief (fig. $5.3 \mathrm{e}$ ).

(85) Id., ibid., p. 208, $\mathrm{n}^{\circ}: 38$.

(86) Iampes identiques au Musee de Narbonne: Ph. Hílisa, Les origines de Narbonne, 19:37, figurées p. 416, fig. 276, $n^{\circ} 1$, type de l'Esquillin, I $^{\mathrm{e}}$ s. av. J.-C. ; an Musie Calvet d'Avignon dans la coll. Calvet, sans provenance :

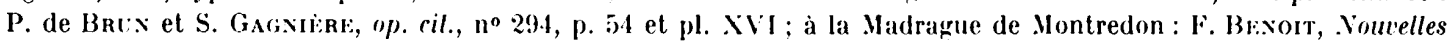
epaves de Provence (III), dans (iallia, X., 1962, p. 161. 
VI. Divers. Fragments de meule en basalte, clous en fer.

VII. Faune. Giros os de breuf, mouton, porc.

Le matériel archéologique de ce puits s'échclonne du II $^{\mathrm{e}} \mathrm{s}$. av. J.-C. (lampe campanienne) au $\mathrm{II}^{\mathrm{e}} \mathrm{s}$. après (sigillée de l'Est) avec prédominance de céramique de la $2^{\mathrm{e}}$ moitié du ier s. av. J.-C.

\section{PLITS $x^{0} 8$}

Carrière Thiébold (juillet 1947). La terre éboulée de la partie supéricure a donné :

I. Polerie fine. A. Protocampanien : 4 petits fragments de coupelles à vernis noir très brillant.

B. Campanien $A$ : fragments de pieds et panse de 3 écuelles.

C. Campanien C: : fragment de plateau.

D. Imitation de Campanien : 10 fragments de 3 écuelles à vernis marron foncé.

E. Céramique à enduit rouge lie de vin intérieur : fragment de bord épais de grande jatte.

F. Pré-sigillée : fragment de jatte à collerette et de bord d'écuelle.

G. Sigillée. a) Italique : tesson orné d'un échassier (fig. .54 a), fragment de panse de tasse guillochée (forme du puits $\mathrm{n}^{0} 7$ ), bord d'assiette f. Haltern I, 2 fragments de bol f. Drag. 46 à bords évasés.

b) Sud de la Gaule : bol tronconique f. Drag. 33, a vernis rouge brillant estampillé : OF. VIT: ILI (atelier de Vitalis, potier de la (iraufesenque, période Claude-Domitien 87 (fig. 54 b) et 2 pieds de bols de la même forme, fragment de fond d'assiette f. Drag. 18 marquée OFCO... (fig. 54 c).

Fragment de pelit bol guilloché verticalement (f. Hermet 13).

H. Vases à parois fines : 1 seul fragment de bord de petile urne marron.

I. Céramique lustrée : 1. Pied d'un grand vase (cf. puits $n^{0} 7$ ) et fragments de coupes à pied dont un bord avec trou de réparation.

2. Vase lourné dont la forme archaïque rappelle les vases chalcolithiques, panse à carène basse et bord a lèvre horizontale. L'argile qui le compose est ocre rouge avec petits grains de calcaire, à engobe marron (fig. 54 d).

II. Polerie Commune. A. Urnes : $1^{0} 3 / 4$ du col d'une grande urne à lèvre en bourrelet, évasće, col à épaulement oblique en terre grise à engobe noirâtre, panse rugueuse, avec 2 autres fragments de bords d'urnes de même type.

$2^{\circ} 11$ fragments d'olla a rebord horizontal, 60 fragments de panses et 1 couvercle presque entier.

B. Marmite : 1 fragment de bord et 2 fragments de fonds.

C. Plateau à pain : 1 fragment de fond.

D. Poterie gris fer : pied d'un vase à liquide du type à bec tréflé.

III. Poterie jaune. 13 fragments de 3 morliers à bec de déversement. 3 fragments de cols, 6 fragments d'anses a sillons verticaux, 3 fragments de pieds et 29 tessons de vases à liquide (cf. puits $n^{0} 7$ ).

(87) OsWat.d, ap. cil., p. 310. 


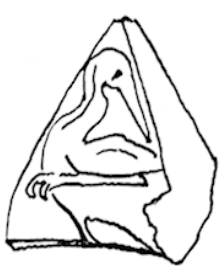

a

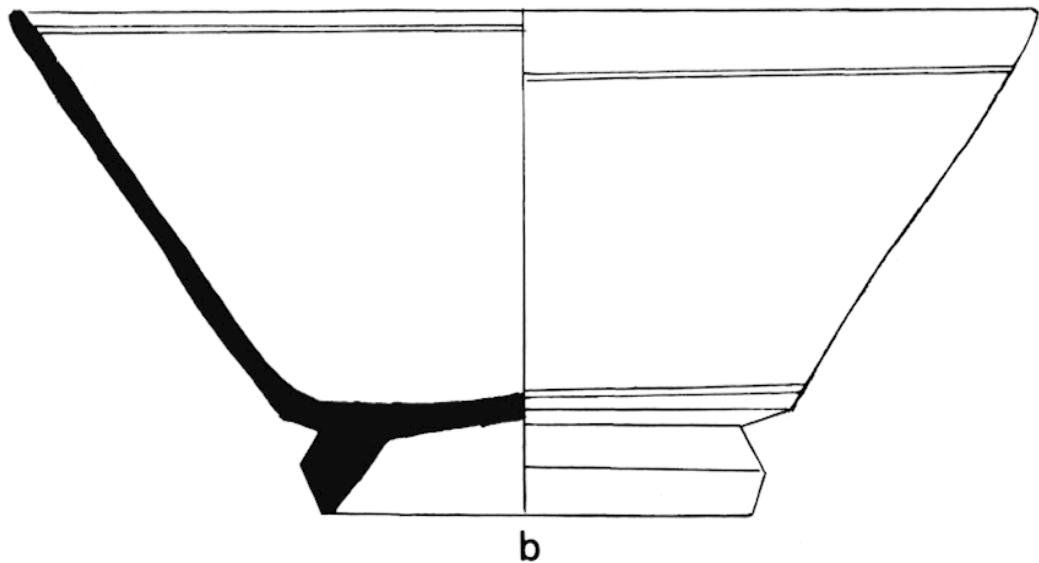

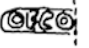

$0.12345^{\mathrm{cm}}$

C<smiles>[3H][IH]</smiles>

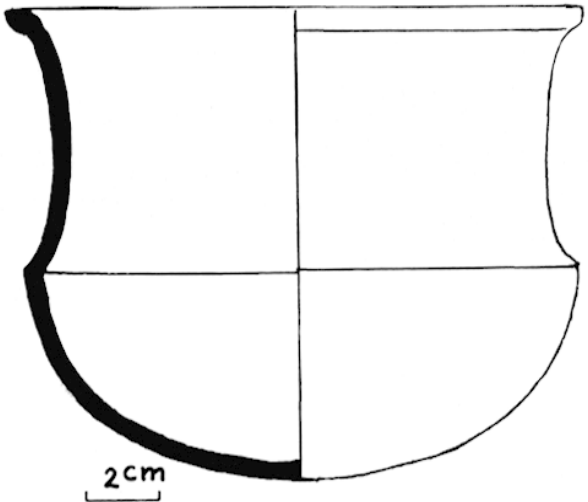

d

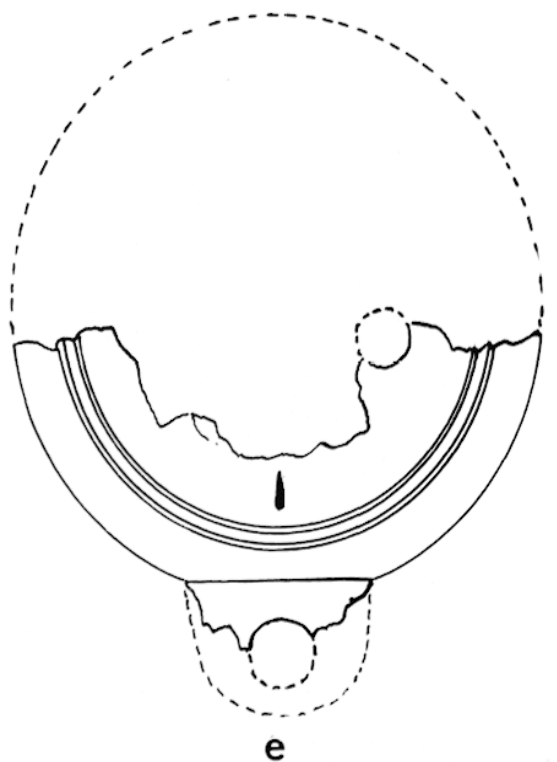

e

54. Puits no 8 . Poterie sigillée, lampe, vase de forme traditionnelle chalcolithique.

IV. Lampe. Partie antérieure d'une lampe en terre jaune à vernis marron avec départ d'un bec rond, type du ${ }_{1}{ }^{\mathrm{e}}$ siècle (fig. 54 e).

V. Divers. 2 fragments de fer oxydé, augette en mollasse blanche de forme irrégulière, fragments de dolium en terre rougeâtre, plaques de revêtement blanc uni.

VI. Faune. Os de bovidés et suidés, hélix.

Remplissage de ce puits datable comme la plupart des autres, de la Tène III avec terminaison au début du $\mathrm{II}^{\mathrm{e}}$ siècle ap. J.-C. 
PUITS No 9

Carrière Thiébold (août 1947).

I. Poterie fine. 1 pied de coupelle en Campanien B (fig. 5̌ a) et 4 fragments de coupes en terre jaunâtre à vernis brun clair (pré-sigillée).

II. Polerie commune. Olla en terre rosée, col court arqué à épaulement, panse galbée, fond plat (H. : $10,8 \mathrm{~cm}$. D. M. : $8,5 \mathrm{~cm}$. D. B. : $5,6 \mathrm{~cm}$.) (fig. $55 \mathrm{~b}$ ). Fragments de fond et panse d'olla de type courant, un couvercle incomplet et 5 fragments de bord. Fond plat de vase en poterie gris-fer.

III. Poterie estampée grise: 2 fragments de bol ornés de palmettes en losange ${ }^{88}$ surmontées de rouelles (fig. $55 \mathrm{c}$ ).

IV. Divers. 1 petit fragment d'anneau de bronze, 1 fragment de fer, 1 silex jaspé et un morceau de matière rouge dure ressemblant à de la cire fondue.

V. Faune. Os de mouton et 5 coquilles d'huîtres.

A part le tesson de poterie estampée, provenant sans doute de la couche de terre de surface enlevée avant le gravier, l'ensemble est du ier siècle av. J.-C:

\section{PUITS $\mathrm{N}^{0} 10$}

Il est apparu sur le front Est d'exploitation de la carrière de M. Bouchet en octobre 1948. Une colonne de décombres et de terre le remplissait sur $11 \mathrm{~m}$. de hauteur et $1,25 \mathrm{~m}$. de diamètre. En dessous, il y avait un vide de $5,50 \mathrm{~m}$. jusqu'à la nappe aquifère, qui se trouvait donc à $16,50 \mathrm{~m}$. de la surface. A quelques mètres au s.-e. de ce puits, des carriers ont mis à découvert, en même temps, à $0,80 \mathrm{~m}$. au-dessous du niveau du sommet de la gravière, un sol de construction s'étendant sur une vingtaine de mètres carrés, formé d'une couche de béton, épais de 6 à $8 \mathrm{~cm}$., parsemé de quelques cubes de mosaïque, reposant sur un lit de pierres brutes posées sur champ.

La stratigraphie du puits $n^{0} 10$, s'établissait ainsi de haut en bas :

Niveau 1. Hauteur $6 \mathrm{~m}$. remplissage de gros galets duranciens, mélangés à des éléments de revêtements muraux formés d'une couche de mortier de chaux et sable blanchâtre à éléments assez gros de $1,5 \mathrm{~cm}$. d'épaisseur, doubléc d'une autre couche de béton fin très mince $(0 \mathrm{~cm} .3)$ avec enduit peint polychrome (bandes et cercles verts, rouges, noirs, bleus, jaunes). Un morceau d'enduit rougeâtre porte une trace de raclure d'or. Aucune poterie.

Niveau 2. Hauteur $3 \mathrm{~m}$.; terre noirâtre avec tessons de poterie.

Niveau 3. Hauteur $2 \mathrm{~m}$. nouvelle couche de fragments de peintures murales en petits morceaux, plus épais que les 1 ers et formés d'une seule couche de béton de $3,5 \mathrm{~cm}$. d'épaisseur avec enduits multicolores : bandes, ponctuations, motifs végétaux (fig. $55 \mathrm{bis}$ ), surmontant un amas de blocs de béton avec par places quelques cubes de mosaïque isolés, provenant sans nul doute de la construction dont on voyait encore les vestiges du sol près de la surface, mais dont les murs avaient disparu. Pas de poteric.

(88) J. Rıgorr, La céramique paléochrélienne sigillée grise, dans Provence hislorique, X, 1960. A rapprocher des fig. 144, p.154, et 167, p.156. 
Céramique du Niveau 2 :

I. Poterie fine. $\mathrm{\Lambda}$. Campanien $\mathrm{A}$ : petit fragment de patère.

B. Pré-sigillée : fragment de paroi de vase en argile rouge pâle, à vernis rouge foncé brillant extérieur.

C. Italique : fragment de bord de bol à lèvre guillochée (fig. 56 a), de panse ornée d'une volute (fig. 56 b), de pied d'assiette, de paroi de tasse à fines encoches (type à 2 anses du puits no 7 ).
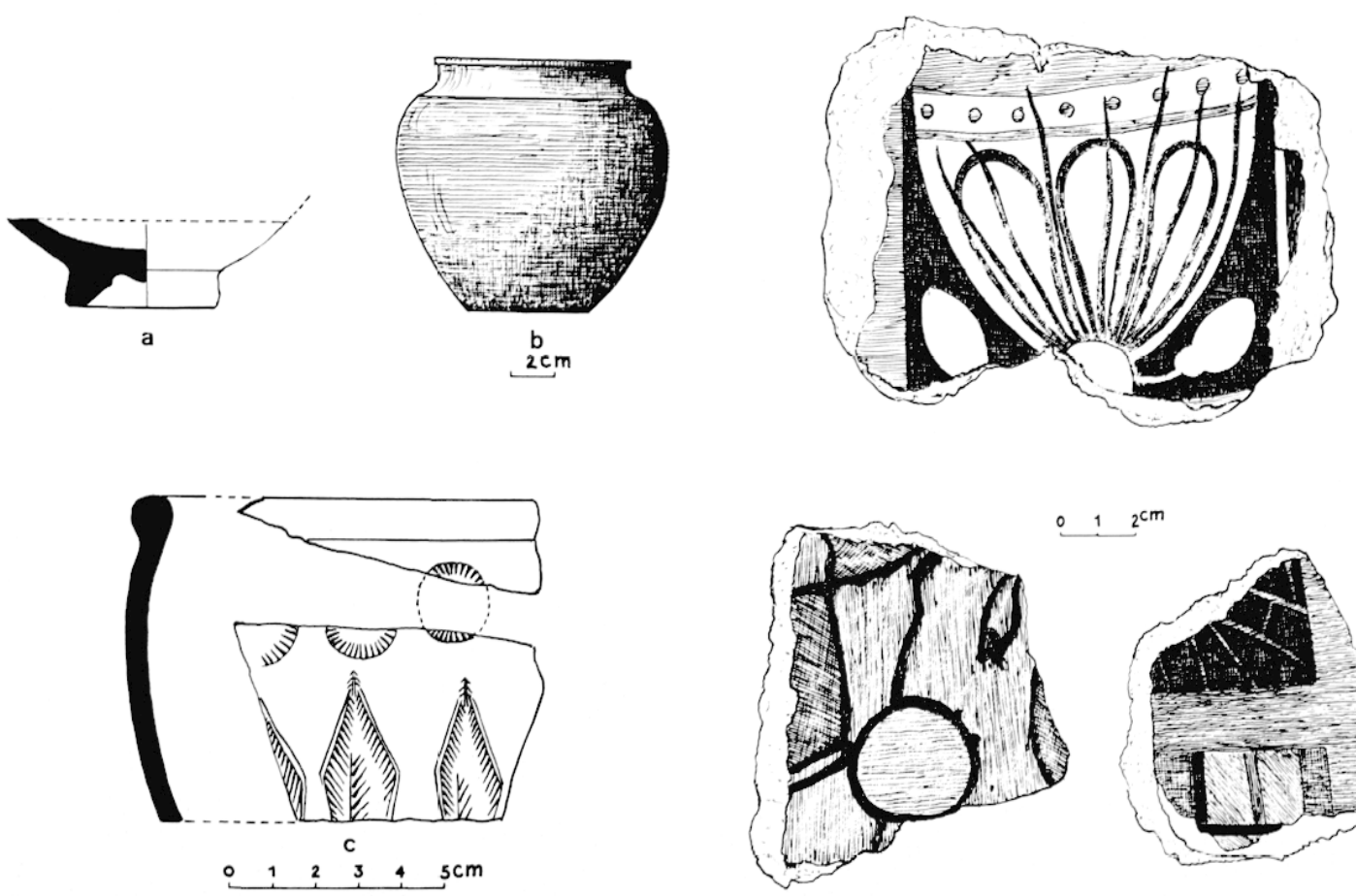

55. Poteries du puits no9.

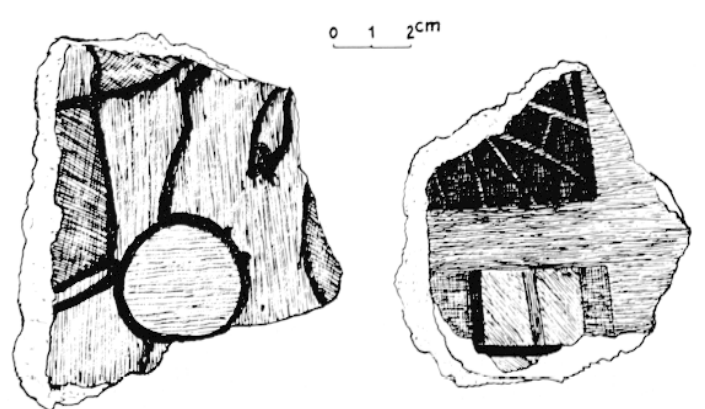

55 bis. Peintures murales du puits n 10.

I. Vases a parois minces : 6 petits tessons appartenant aux formes des vases lisses du puils $n^{\circ} 7$. sauf un petit fragment gris avec triangles allongés barbotinés.

E. Poterie lustrée : 2 fragments de coupes.

II. Poterie commune. A. Urne reconstituée en terre grossière noiràtre, col court à ouverture sans rebord et à épaulement, panse rugueuse, fond plat et large $($ H. : $13 \mathrm{~cm}$. D. $\mathrm{M} .: 17 \mathrm{~cm}$. D. B.:9 cm). (fig. $56 \mathrm{f}$ ). Olla à col oblique lisse et panse peignée (incomplète) et 6 fragments de bords, de même type. Olla en argile rougeàtre (partie inférieure). 34 fragments de panse, 6 de fonds plats et 1 anse de modelé inusité avec appendice vertical en forme de poucier (fig. $56 \mathrm{c}$ ), fragment de grand couvercle et 4 fragments de 3 autres plus petits.

B. Marmite arrondie, à rebord plat sur le dessus (reconstituée en entier), type du puits $n^{0} 7$ (H. : 12,3 cm. D. O. : $30,2 \mathrm{~cm}$ ). Fragment de grande bassine dont la lèvre plate ne porte pas les incisions obliques habituelles, mais des traces de peignage irrégulier (grains de calcite visibles dans l'argile noirâtre.).

C. Polerie gris-jer: Vase à liquide pansu, à bec tréflé (H. : 24,5 cm. D. M. : 22,5) cm. D. B. : $7 \mathrm{~cm})($ fig. 57 a). 
D. Polerie jaune: 2 vases à liquide à bec allongé et anse torsadée (type du puits $\mathrm{n}^{0} 7$ mais moins larges.). L'un mesure $31 \mathrm{~cm}$ de haut et $20 \mathrm{~cm}$. de diamètre maximum (fig. $57 \mathrm{~b}$ ), l'autre, $21 \mathrm{~cm}$. et 16.110 tessons de vases à liquide divers et 1 petite anse simple. 1 fragment de bord de mortier.

III. Lampes. 1. Lampe du $1^{\text {er }}$ s. ap. J.-C. en terre jaune clair dure, à couverte marron "praline " brillant, cuvette très creuse avec rosace à 25 rayons incurvés autour du trou central ${ }^{89}$, fines volutes très saillantes : le bec, cassé, était probablement angulaire (fig. 56 e) (f. Dressel. 9).

2. Autre lampe incomplète de même type à couverte brune, ornée sur le dessus d'un enfant courant à gauche, poursuivi par une chèvre. Volutes très détachées (fig. 56 g).

3. Fragment de dessus de lampe orné : pieds et bras humains (fig. $56 \mathrm{~d}$ ).

4. Fragment de réservoir et de bec angulaire.

IV. Métal. Fragment de plaque de bronze et d'instrument en fer, informe.

V. Faune. Os de bœuf, mouton, porc.

Le comblement de ce puits comporte exceptionnellement, parmi les décombres d'une habitation gallo-romaine voisine, une grande quantité de fragments de peinture murale se rapprochant du "premier style ", avec intercalation de céramique d'époque augustéenne.

PUITS No 11

Carrière Bouchet (juin 1949).

I. Polerie fine. A. Campanien, imitation régionale de la forme 36 du Camp. $A$ (rebord d'écuelle à marli ${ }^{90}$, fragments de coupelles et d'écuelles à vernis mat, de vases en terre jaune clair à vernis brun. 2 fragments de bord de plateau et de panse avec départ d'anse en imitation Camp. C.

B. Pré-sigillée : fragments de col à 2 sillons parallèles et collerette intérieure (fig. 58 a) et de coupe à panse très arquée avec rainure tout le long du bord (fig. $58 \mathrm{~b}$ ).

C. Sigillée : 4 fragments de formes lisses peu reconnaissables. 1 fragment tronconique et 1 petit tesson orné (fig. $58 \mathrm{c}$ ).

D. Poterie à engobe marron : partie supérieure d'une marmite à anse courte, bord évasé et parois peu épaisses, en argile dure, fine, blanchâtre, avec léger enduit marron clair peu adhérent (fig. $58 \mathrm{~d}$ ).

E. Poterie lustrée : fragments de 2 jattes basses, en argile rose lissée à l'intérieur et au bord, l'extérieur étant sans engobe (peut-être une imitation locale de la céramique à enduit intérieur lie de vin, importée d'Italie).

II. Polerie commune: $\Lambda$. Urnes : 11 fragments d'olla du type de la Tène (3 ex.) et 1 fragment à bord en bourrelet sans encoches au col. 1 fond de couvercle, et fragments de 3 autres.

B. Plateau circulaire à pain : 1 fragment de bord.

(89) Décor semblable sur un dessus de lampe trouvé à Glanum: P. de Brcx, Les lampes antiques en argile du Musée des Alpilles à Saint-Rémy-de-Provence (B.-du-R.), Nìmes, 1933, pl. VII, no 9, et aussi sur une lampe provenant de Vaison au Musée Calvet: P. de BrCn et S. Gagnitre, op. cit., p. 47, no 211 et p. 48, pl. XIII.

(90) N. Lambogilia, Apuntes sobre Gronologia ceramica, pl. IV. 

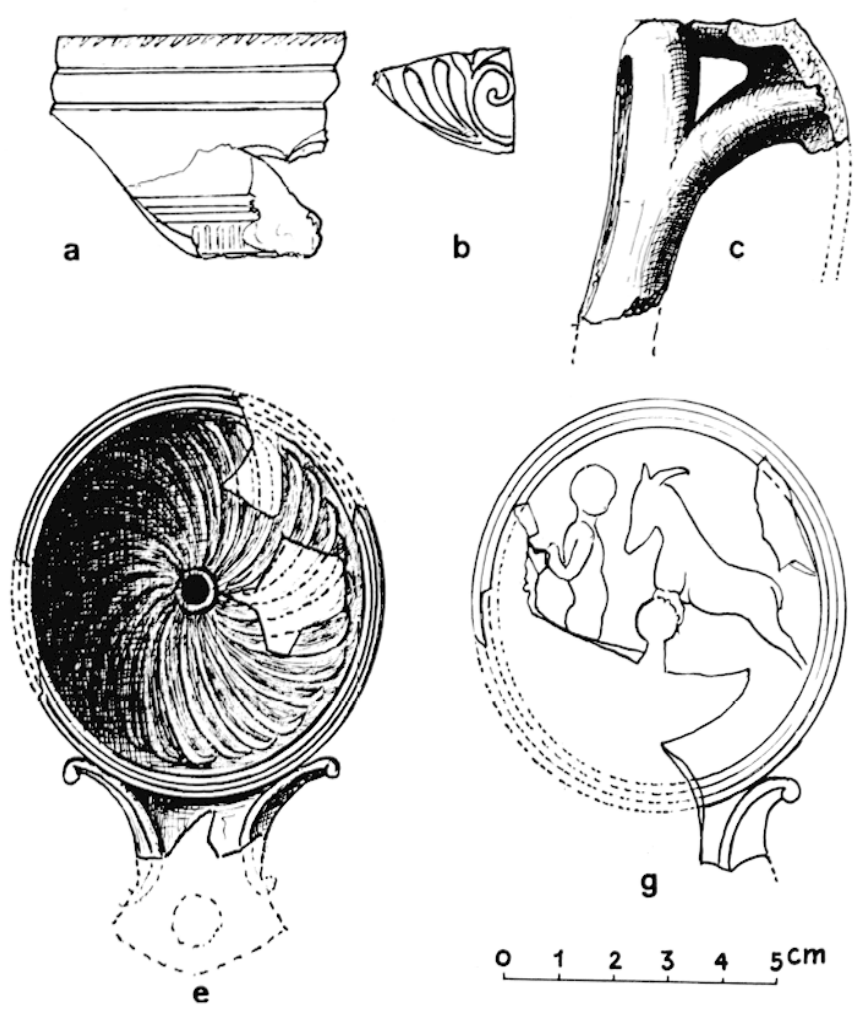

$0 \quad 12345 \mathrm{~cm}$

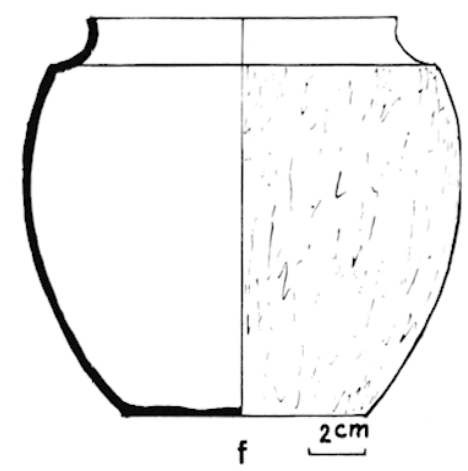

56. Puits no 10. Poterie sigillée, anse, urne et lampes.

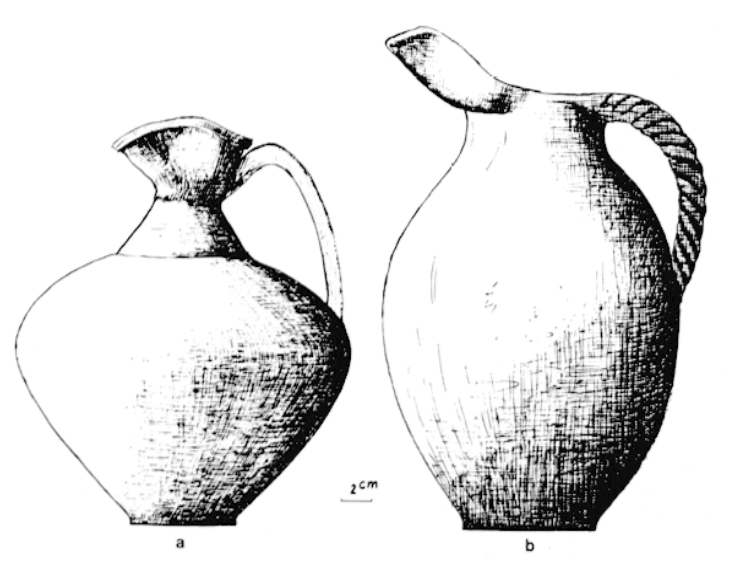

57. Puits $n^{\circ} 10$. Vases à liquide en poterie commune.
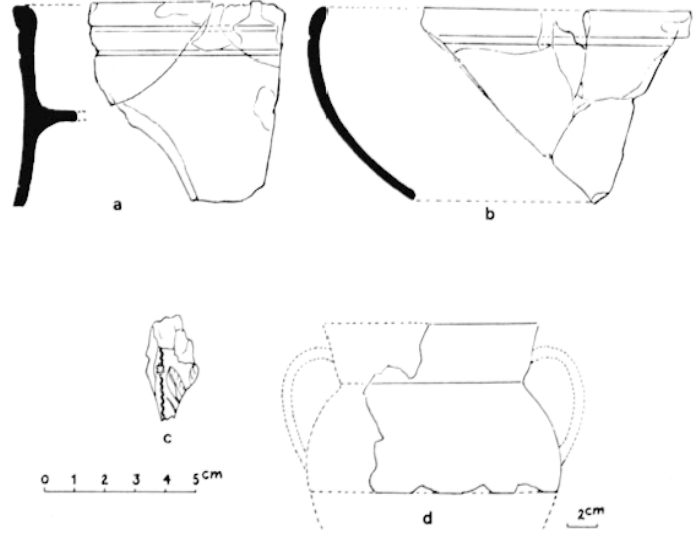

58. Matériel du puits no 11 .

C. Bassine : 15 fragments d'un seul récipient avec anse en oreille.

III. Polerie jaune. 1 goulot de vase à liquide (type du puits $n^{0} 7$ ), fragments de morliers.

IV. Faune. Os de bouf, mouton, cerf élaphe.

Ensemble homogène de la $2^{\mathrm{e}}$ moitié du $\mathrm{I}^{\mathrm{er}}$ s. av. J.-C. 
PUI'TS $N^{0} 12$

Carrière Bouchet (juillet 1955). La colonne de terre de remplissage, éboulée à la suite d'un coup de mine, avait $4 \mathrm{~m}$. de haut et 1,30 m. de diamètre. En-dessous, le puits était comblé avec des galets de la Durance. La couche de terre de $4 \mathrm{~m}$. a donné des vestiges se rapportant, comme le puits précédent, au ${ }^{\mathrm{er}} \mathrm{s}$. av. J.-C.

I. Polerie fine. A. Protocampanien : fragment de panse de coupe.

B. Campanien 13 : petit tesson à bande blanche peinte intérieurement.

C. Imitation de Campanien, probablement massaliote : 7 écuelles incomplètes de même type dont 3 fonds avec graffites extérieurs (fig. 59 a b c). livvre.

D. Campanien C: : fragment de plateaux (bords et fonds) et de bol, forme I, a 2 sillons sous la

E. Imitation de Campanien C : écuelle incomplète, 2 fonds et 2 fragments de patère.

F. Vases à parois minces : Urnette de forme ordinaire en argile bistre clair. Fond de vase lisse, pied de gobelet orné de guirlandes de perles, panse barbolinée, fragment d'unguentarium à pied allongé.

G. Poterie peinte de Lezoux : petits tessons à engobe marron foncé et fragment de vase peint à décor géométrique (bandes marron sur fond blanc (fig. $59 \mathrm{~g}$ ).

II. Poterie lustrée. 6 fragments de jattes. types du puits $n^{0} 7$.

III. Polerie commune. 1 . Ollae de la Tène $: 4$ reconstituées en entier, 6 incomplètes et fragments d'une vingtaine d'autres.

B. Ollae à bourrelet à la base : 1 reconstituée et fragments de 3 autres.

C. Ollae à 1 anse : 3 incomplètes.

D. Ollae a 2 anses et panse lisse : 1 reconstituée et fragments de 2 autres, dont une porte sur le col le graflite : $\mathrm{H}+($ fig. $59 \mathrm{~d}$ ).

E. Couvercles d'ollae: fragments d'une quinzaine.

F. Plateau à pain : 1 seul fragment.

G. Jattes à goulot court : une dizaine, incomplètes.

IV. Poterie jaune. A. Vases à liquide à panse large bi-tronconique, col élevé, étroit, bord évasé à lèvre oblique rentrante, anse à sillon dorsal, pied creux $($ H. : $13,3 \mathrm{~cm}$. D. M. $13,4 \mathrm{~cm}$. D. B. : $6 \mathrm{~cm}$. i (fig. 58 i).

B. Vase de même type, à enduit marron clair extérieur.

C. Fragments de plusieurs vases à liquide (forme du puits n ${ }^{0} 7$ ), 2 tessons avec graffite (fig. $59 \mathrm{ef}$ ).

D. Fragments de panses d'amphores avec traces de matière résineuse calcinée à l'intérieur.

V. Lampes. Fragment d'une lampe de type delphinö̈de en terre jaune à couverte marron clair brillant, bec rectiligne, perlée sur le dessus (fig. $59 \mathrm{~h}$ ).

VI. Divers. Fragments de fer très oxydé.

VII. Faune. Os de bœuf (abondant) mouton, porc. 

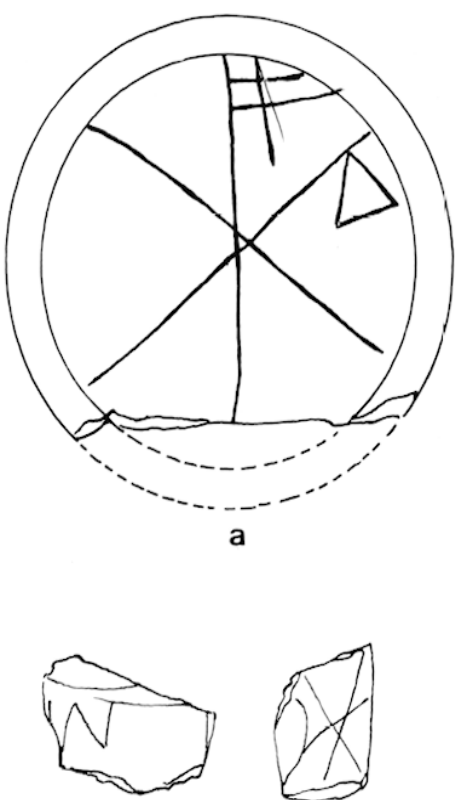

e

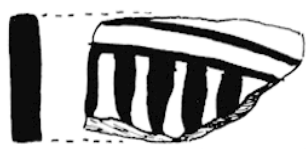

9
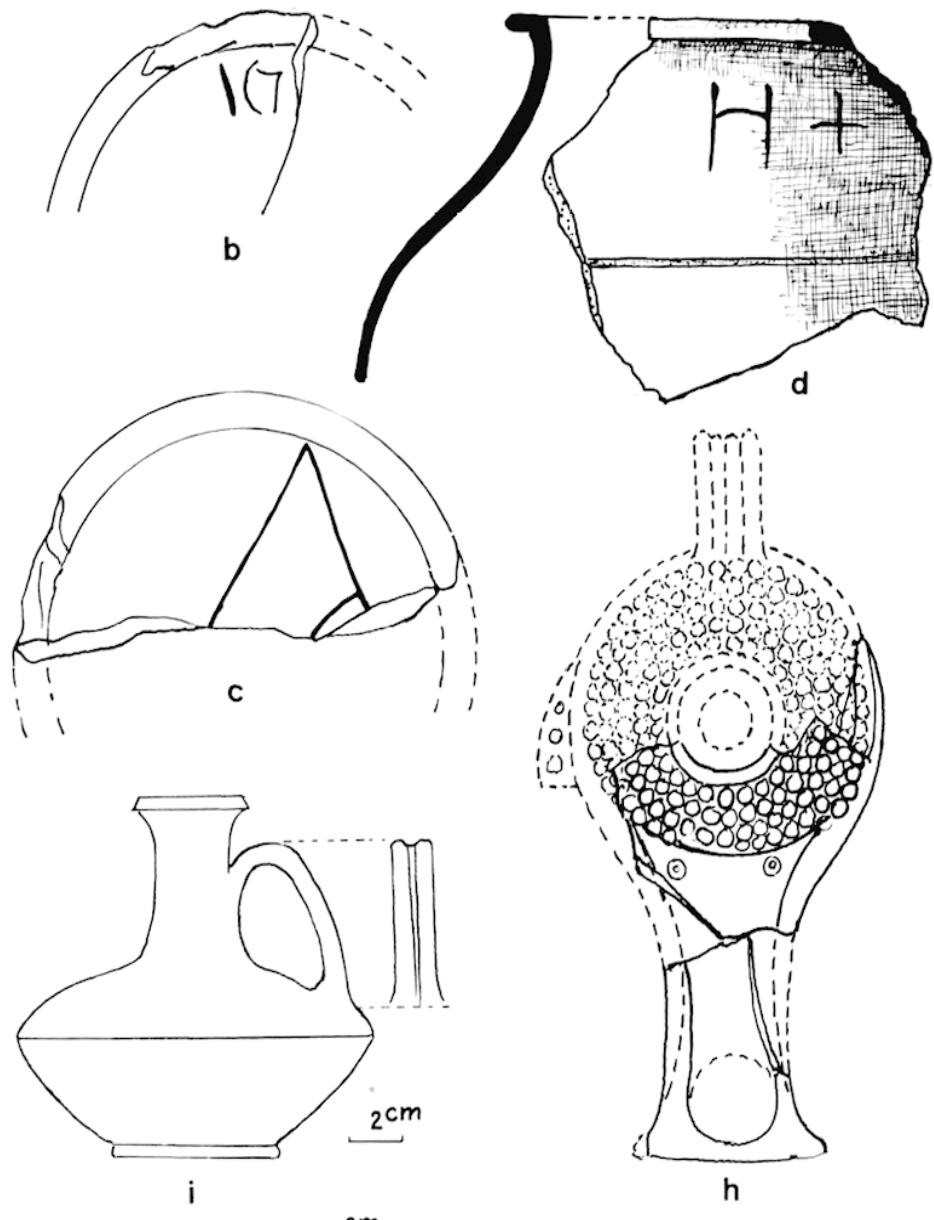

$0 \quad 12345^{\mathrm{cm}}$

59. Puits $n^{\circ} 12$. Graflites, lampe delphinoïde, vase à liquide, fragment de vase peint de I.ezoux.
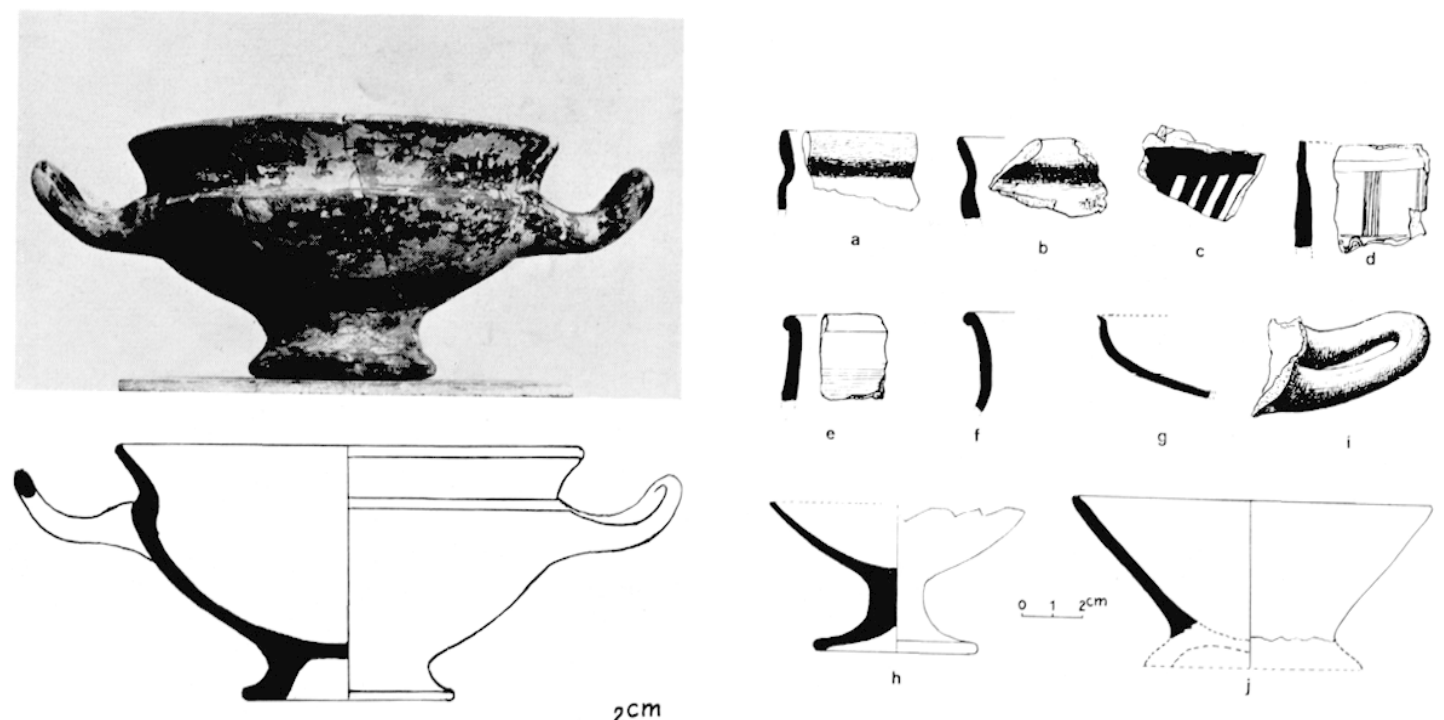

61. Puits no 13. Céramique imitant les formes des 60. Puits no 13. Coupe en poterie imitation phocéenne. vases phocéens. 
PUITS N0 13

Carrière Bouchet. Découverte en juillet 195̄. La terre de remplissage était particulièrement grasse et charbonneuse. Elle contenait un matériel archéologique du début du $2^{e}$ Age du Fer, daté par des fibules caractéristiques.

I. Céramique d'importation (imitation massaliote des types d'Asie mineure). a) 2 fragments de bord oblique, peu élevé, sur panse arrondie de coupes à anses horizontales, de tradition ionienne (fig. $61 \mathrm{a} \mathrm{b}$ ), en argile pàle à enduit marron très clair intérieur et extérieur ; 2 fragments de panse, plus épais et un tesson orné de bandes peintes foncées (fig. $61 \mathrm{c}$ ).

b) Coupe à 2 anses horizontales coudées, bord oblique et pied creux profond, en argile grise tendre, à engobe gris foncé écaillé par places ${ }^{91}$ (H. : 9,4 cm. D. 0. : $19 \mathrm{~cm}$.) (fig. 60) ; pied de vase semblable ; petit tesson à ondes làches ; fragments de col à traits verticaux espacés sur zone d'ondes serrées (fig. $61 \mathrm{~d}$ ), de bord droit à sillons parallèles (fig. $61 \mathrm{e}$ ), de bord arqué (fig. $61 \mathrm{f}$ ), de lèvre d'oenochoé, de coupe arrondie (fig. $61 \mathrm{~g}$ ).

II. Poterie commune. A. On reconnait certaines formes en terre cuite lustrée qui imitent les coupes massaliotes, notamment un pied à tige élevée (fig. $61 \mathrm{~h}$ ), une anse latérale arrondie (fig. $61 \mathrm{i}$ ), un fragment de bord à ressaut, et un bol à pied creux (fig. $61 \mathrm{j})^{92}$.

B. D'autres formes également lissées avec soin sont bien caractéristiques de l'Hallstattien final ; plusieurs vases ont pu être reconstitués totalement :

1. Coupe à pied large, bas, légèrement évidé, d'un très beau noir lustré $(\mathrm{H} .: 8,6 \mathrm{~cm} . \mathrm{D} .0$. : D. $0 .: 24,4 \mathrm{~cm}$.) (fig. 62 a et $63 \mathrm{a}$ ).

2. Coupe de mème type, mais à fond plat (H. : $9,7 \mathrm{~cm}$. D. $0 .: 24,8 \mathrm{~cm}$. (fig. $63 \mathrm{~b}$ ), ainsi qu'une autre incomplète.

3. Écuelle à panse tronconique renversée, fond plat et bord légèrement rentrant $(\mathrm{H} .: 10,8 \mathrm{~cm}$. D. $0 .: 23,4 \mathrm{~cm}$.) (fig. $62 \mathrm{~b}$ et $63 \mathrm{c}$ ).

4. Écuelle plus petite, à bord caréné (H. : $6,3 \mathrm{~cm}$. D. $0 .: 13,4 \mathrm{~cm}$.) (fig. $62 \mathrm{c}$ et $63 \mathrm{~d}$ ) et fragments de 4 autres semblables ${ }^{93}$.

C. Urnes. Comme d'habitude ce sont les récipient les plus nombreux. Il convient d'en distinguer 3 catégories :

a) Urnes de facture soignée quoique non tournées, en argile bien cuite, dure, sonore, à petits grains de calcaire, parois plutôt minces, cols lissés de couleur noire luisante, panse peignée :

1. Petite olla de forme harmonieuse, bord à lèvres évasées, fond plat, ligne d'arrêt entre le col et le haut de la panse (H. : $14,2 \mathrm{~cm}$. D. $0.10,4 \mathrm{~cm}$. D. B. : 6,2 cm.) (fig. 62 e et 63 1) et 7 autres incomplètes.

2. Parlie supérieure d'une grande urne (D. $0 .: 25,3 \mathrm{~cm}$.) de même type, avec bordure de petites encoches à peine marquées en bas du col, et un autre fragment à encoches fuselées.

3. Fragment d'urne à anse en double boudin vertical. p. 65.

(91) "Bucchero gris" local, sans décor, de F. Villann, La céramique grecque de Marseille (VI $I^{\mathrm{e}} I V^{\mathrm{e}}$, s.), 1960,

(92) A rapprocher des "petites coupes sur pied " découvertes à la Roque : P. LARDERET, L'oppidum préromain de la Roque, commune de Fabrègues (Hérault), dans Gallia, XV, 1957, p. 16, fig. 11 (suite), nos 16, 17 et 19.

(93) Récipients identiques à la Roque : P. Larderet, op. cil., p. 15, fig. 11, nos $1,2,3$. 


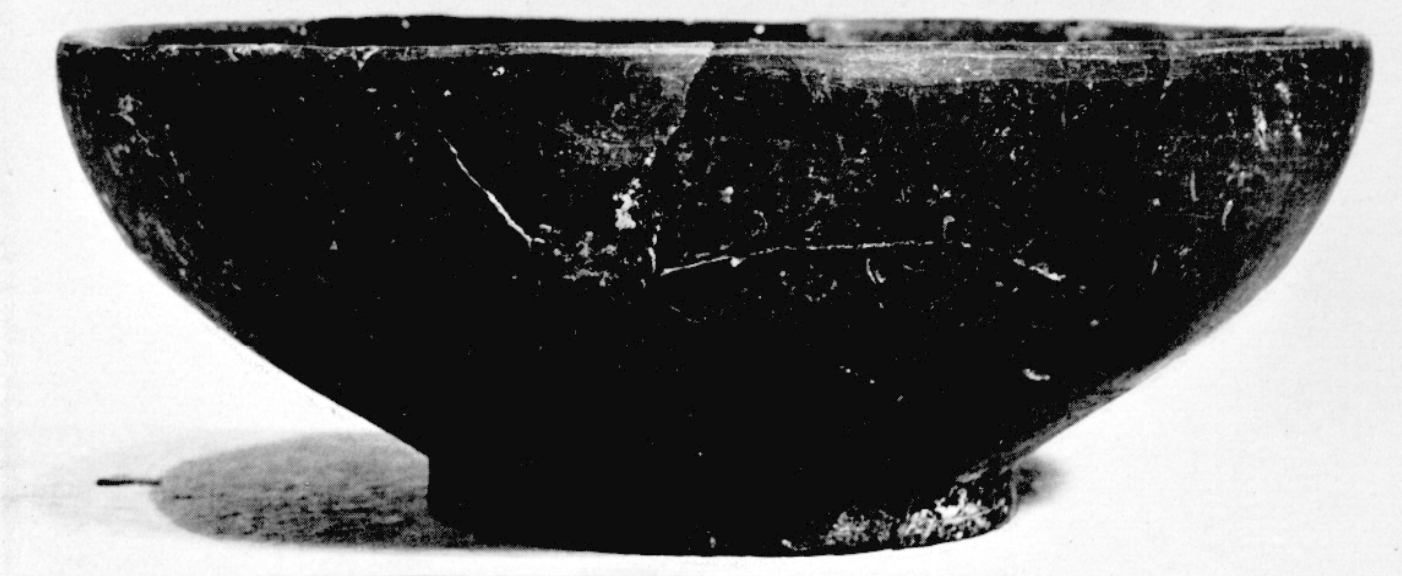

a
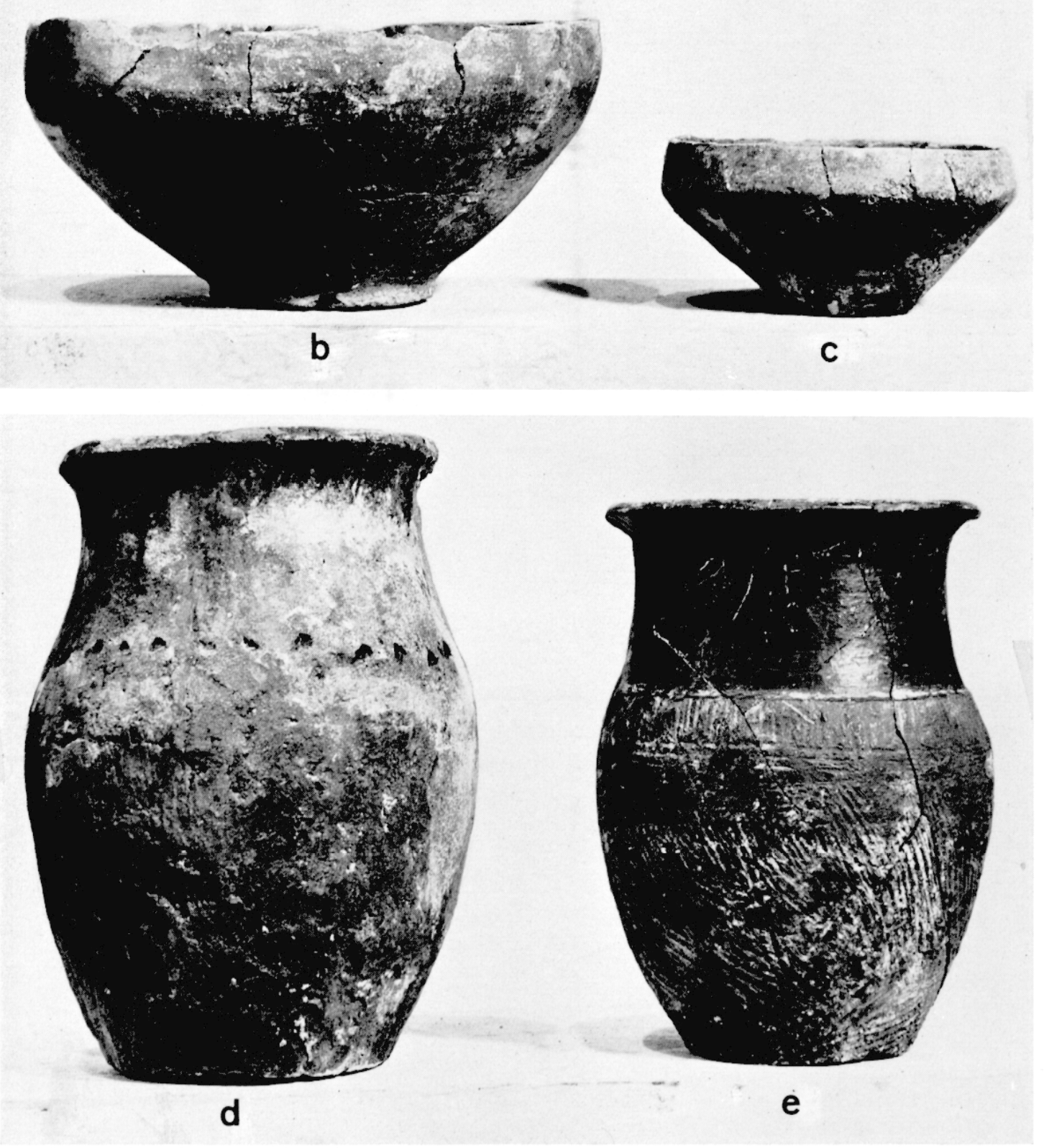

6:. Puits $n^{0}$ 13. Vases indigrines (coupes et urnes). 
b) Urnes en argile grossière, mal cuite, à gros grains de dégraissant, parois épaisses, fond plat et panse peignée : une olla avec rangée de petites encoches au bas du col (H.:16 cm. D. $0.10 \mathrm{~cm}$.) (fig. 62 d et 63 f), 2 incomplètes, et 200 tesssons appartenant à une quinzaine de ces récipients dont le haut de la panse est orné de différents motifs, en creux : rangée de chevrons emboîtés (fig. 64 a). petits cercles (fig. $64 \mathrm{~b}$ ), empreintes coquillères de gastéropodes (fig. $64 \mathrm{c}$ ).

(. Urnes de conlenance plus grande que les précédentes :

1. Partie supérieure à rangée de lignes brisées au bas du col (D. 0. : $24 \mathrm{~cm}$.) (fig. $65 \mathrm{a}$ ).

2. Partie supérieure de 2 autres vases de même type, sans décor (D. 0. : 20,5 (cm.) (fig. 6.) b).

D. Jattes. Fragment d'une grande jatte lissée intérieurement, peignée à l'extérieur, de profil oblique (fig. $64 \mathrm{e}$ ) et fragments de 4 autres : 2 a encoches sur le dessus du bord, 2 autres avec bec de déversement rudimentaire obtenu par pincement de l'argile encore molle (fig. $64 \mathrm{~d}$ ).

E. Polerie jaune. Fragment d'un grand vase pansu à parois asse\% minces, dures, dont il est difficile de reconnaître la forme.

F. Amphores. Fragment de goulot et de panse de 2 amphores massaliotes à pàte rosée micacée.

G. Divers. 2 disques en lerre cuite, pris, l'un dans la paroi d'un vase lissé en terre grise, l'autre en argile rougeâtre.

III. Fibules en bronze. Eilles sont toutes du type marnien ou de la Tène ${ }^{94}$ :

1. Fibule à arc régulier de section circulaire, prolongé par un porte-agrafe coudé et replié, à renflement terminal allongé s'appuyant contre l'arc : ressort à 7 spires à corde interne $(\mathrm{I}$. : $6 \mathrm{j}$ cm) (fig. 66 a). Cette fibule a été trouvée au mème niveau que la coupe massaliote grise décrile plus haut.

2. Fibule d'une forme particulière, dite à faux-ressort. Porte-agrafe relevé el coudé, terminé contre l'arc par un enroulement en ressort à double spire qui n'a qu'un rôle ornemental ${ }^{95}$ (L. : $5,3 \mathrm{~cm}$. (fig. $66 \mathrm{j}$ b).

3. Petile fibule a ressort unilatéral d'une seule spire, porte-agrafe simple, à prolongement aplati replié sur l'are ${ }^{96} \mathrm{~L}$. : : :3 cm, ò) (fig. $66 \mathrm{c}$ ).

4: Fibule dont il manque l'ardilllon el la plus grande partie du ressorl. I. : $4 \mathrm{~cm}, 8$ (fig. $6(6 \mathrm{~d}$ ).

IV. Fanne. Os de bouf, mouton, porc.

Le remplissage de ce dernier puits a une grande analogie avec celui du no 1 . (On peul y noter la même rareté de la céramique d'importation, provenant sans doute de Massalia et l'abondance de la poterie indigène qui présente ici une dualité de facture nettement caractérisée. La datation de l'ensemble est bien donné par les fibules typiques de Ia Tène I (500-400 av. J.-C.) ou La Tène Ancien, ce qui permet de constater une fois de plus, le prolongement de Hallstatt II qui "empiète largement sur la période de La Tène » ${ }^{97}$, ainsi qu'une certaine perduration dans l'imitation régionale de la céramique éolienne, qui a pu, dans l'intérieur, persister jusqu'au seuil de l'époque hellénistique ${ }^{98}$.

(9.1) Fibules à appendice caudal relevé, sans décor plastique : Dícher.FTrti, . Manuel, II p. 1250 et figr. 5333.

(95) Denise Bretz-Malubr, Les fibules à faux ressorl du .Musée de Chàlons-sur-Marne, dans Bull. de la soc. préhist. fr., I.VI, 1959, p. 448. A rapprocher d'une fibule provenant de Chouilly les Jogasses, p. 4.19, pl. I, fig. 1 mais dont le ressort e actif " est à corde externe. La notre serait plutiot à dater de la Tène I b.

(96) Type de fibule rencontré également dans les tombes marniennes : Chanoine FavrET, La nécropole gauloise de Villesenen.r: (canton de Vertus, Marne) dans Bull. de la soc. préhist. fr., XI.VII, 1950, p. 443, fir. 9.

(97) .J.-J. H Атт, De l'Age du Bronze à la fin du I er Age du Fer, problimes el perspeclives de la Prolohisloire française, dans Bull. de la soc. préhisl. fr., I, I, 1954 , p. 109.

(98) li. Viltara, op. cil., p. 67. 

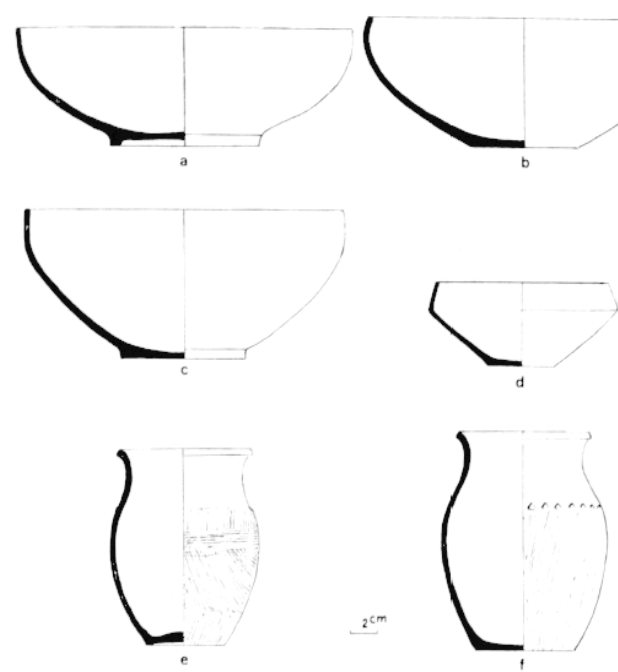

63. Puits $n^{\circ} 11$. Vases indigènes.

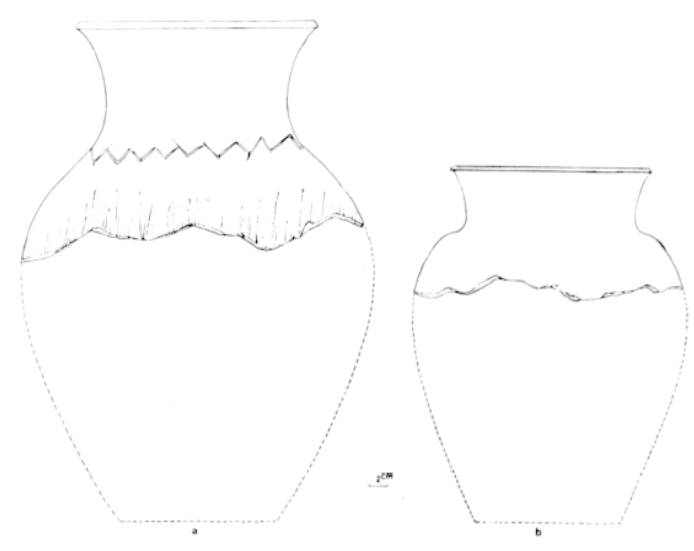

65. Puits $n^{\circ} 13$. Vases hallstattiens.

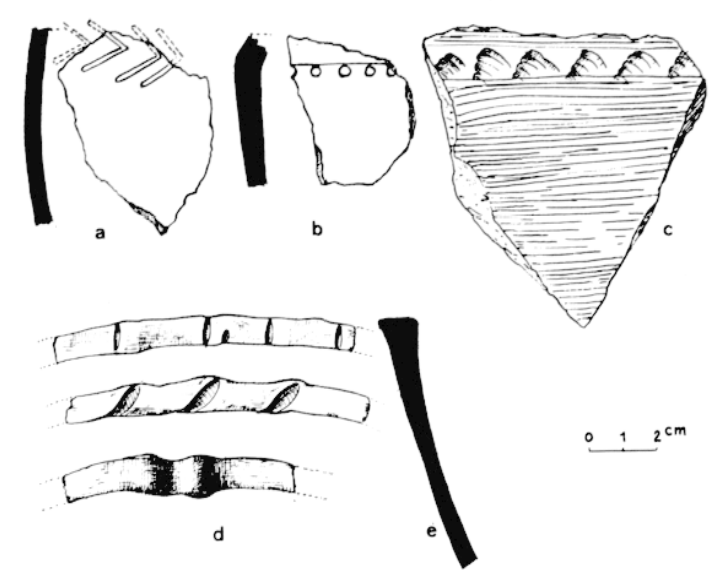

64. Puits $n^{\circ}$ 13. Tessons ornés (poterie indigène).
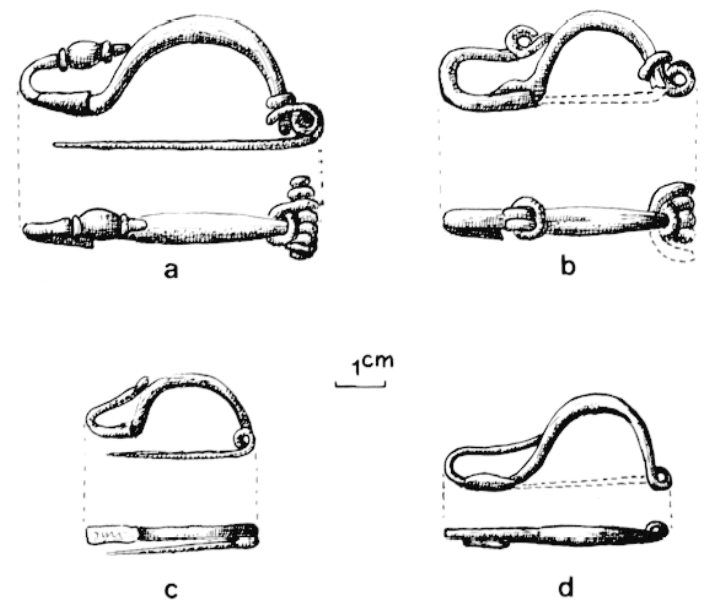

66. Puits no 13. Fibules en bronze (type de La Tène I).

\section{Les fosses-silos}

FOSSE $N^{0} 1$

La $1^{\text {re }}$ fosse, découverte en septembre 1941, se trouvait dans la carrière Thiébold entre les puits $\mathrm{n}^{\mathrm{os}} 4$ et 5 (fig. 50 ), à $4 \mathrm{~m}$. du $1^{\mathrm{er}}$ et $6 \mathrm{~m}$. du $2^{\mathrm{e}}$ Son creusement ancien avait été arrêté à $4,60 \mathrm{~m}$. de profondeur, un peu au-dessus du niveau de base de la carrière. Assez étroite $(0,90 \mathrm{~m}$. de diamètre) elle était comblée par une terre noirâtre sableuse. 3 couches distinctes :

$1^{0}$ couche supéricure de $2 \mathrm{~m}$. d'épaisseur, qui s'est éboulée lors de la découverte;

$2^{0}$ couche moyenne de $1 \mathrm{~m}$. d'épaisseur de sable argileux, compact, stérile.

$3^{\circ}$ couche inféricure de $1,50 \mathrm{~m}$. jusqu'au fond de la fosse, qui contenait des pépins de raisins. 


\section{Conche supérieure}

I. Polerie fine. A. Fragments de bord de coupe proto-campanienne. de pied de vase en Camp. A., de plateau en Camp. B, et de paroi de vase en imitation Campanienne C:.

B. Terra-sigillata :

a) Italique : 1. Beau vase campaniforme, moulé, orné (f. I)rag. 11), à vernis rouge coralin très vif, dont il manque le pied et quelques fragments de la partie supérieure (Haul. actuelle : $12 \mathrm{~cm}$. diam. ouverture : $18,5 \mathrm{~cm}$.) (fig. 67 et 68 ). Le décor, cmprunté à la $I_{y}$ thologie grecque, représente un combat entre Grees ct Amazones; il se développe tout autour du vase entre un couronnement d'oves, souligné par une rangée de perles en haut at une frise de casques au voisinage du pied. La scène principale de cette amazonomachie parait être celle qui représente un guerrier grec de face. largement fendu en arrière, tentant d'entraîner, en la tirant par ses vètements, une amazone qui agrippe une statuette de divinité. sur socle, à la droite de laquelle se dresse un autel, plus large que haul, surmonté d'un grand candélabre élancé, voisinant avec une paire de cymbales; au pied de l'autel, une longue torche allumée est renversée. Le guerrier grec, casqué, revêtu de la lorica, armure moulant le tronc, derrière laquelle flotte la chlamyde est armé d'un bouclier rond (clipeus) dans lequel est passé son bras gauche dont la main tient une sorte de courte épée. La guerrière scythe tîte nue, les cheveux courts, porte une tunique blousante serrée à la taille, ses jambes sont qainées de hauts chaussons. L'attitude des deux personnages de cette scène fait penser tout de suite au thème bien connu d'Ajax et de Cassandre, sa similitude aver la représentation rlassique de cet épisorle de la guerre de Troie étant frappante ${ }^{99}$. Pourtant, la figure féminine qui enlace la statue, est exartement semblable par ses vêtements et son allure. aux adversaires des guerriers grees que l'on voit à la suite sur notre vase. ot n'a rien dess atours de la prophétesse. Quant à la divinité dont le sanctuaire vient d'ètre profané. il s'agirait non pas d'Athéna, mais plutòt d'Artémis coiflée du polos, déesse dont les Amazones étaient prêtresses ou adoratrices; les attributs qui l'accompagnent : candelabrum. cymbalum. fax, ora, font penser à l'Artémision d'Éphèse ${ }^{100}$. Ce cas n'est pas isolé d'une représentation de scène d'amazonomachie où l'attitude des personnages crée des illusions parce qu'clle. s'apparente, pour la forme, à la scène des violences faites par le chef locrien à la fille de Priam, pendant le sac de Troie. Juliette Davreux met l'accent sur ces ressemblances "sources de doute et de confusions pour l'interprétation" "101.

A gauche de cet ensemble, 2 Amazones semblent voler au secours de leur compagne, l'une à pied, armée l'un arc. courant en avant, le bras droit tendu, l'autre, à cheval, casquée. avec bouclier rond it lance en arrêt ${ }^{102}$. Ensuite ce sont des corps a corps entre (irecs et

(99) Juliette Innéx, La légende de la prophélesse Cassandre, dans Bibliolhique de la Fac. de Philo. el Lellres de

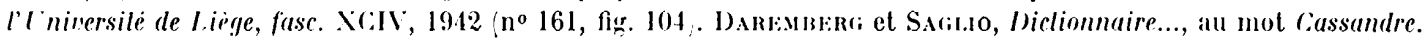

(10): Plixe, Misl. nat., XXXIV, 53.

101 Juliette Dorra:x, op. cil. Voir notamment : p. 166, no 103, un relief découvert en 1931 lors des fouilles, pratiquées au Pirie copie de style italique d'un original grec de l'épo(que de Phidias) et encore: p. 17:2, n²115, p. 17, $n^{\circ} 117$, p. 198, n० 169.

102) Von Bormuk, Amazones in Greek Arl, Oxford 1957: attitude semblable sur des vases attigues a figures rouges, pl. I.NXVit. 


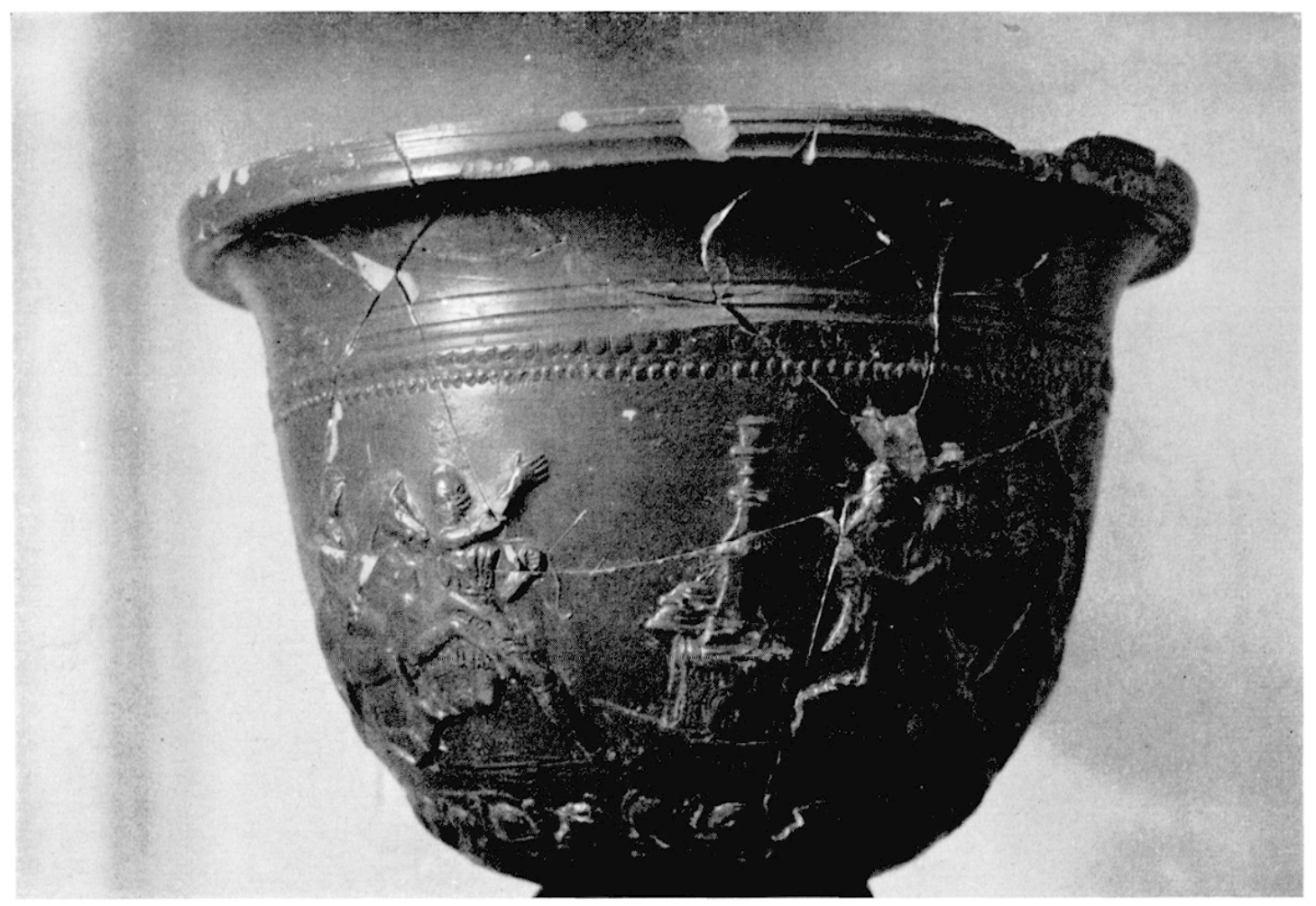

67. Fosse $\|^{\circ}$ I. Vase armi du potier lineius Aleius, atelier d'Arezo Italie.

Amazones, l'une de ces dernières combattant le sein nu' ${ }^{103}$. Cà et là, sont disposés des armures, houcliers, casques, lances, jonchant le sol.

La frise qui souligne le décor est intéressante par la composition el la disposition de ses éléments : un casque à cornes latérales et pare-joues, vu de face, entre 2 autres casques à courte crête, avec visière, couvre-nuque laissant une ouverture pour les oreilles, et paragnathides, de profil, succession qui se répète 8 fois autour du vase. Le casque à cornes est de type gaulois, bien connu par ses représentations sur les ares triomphaux de la Narbonnaise (Saint-Rémy, Orange) et aussi par le bas-relief de la Brague au Mlusée d'Antibes ${ }^{104}$. Tandis que les autres sont des casques de légionnaires romains ${ }^{105}$. Cette association et cette disposition ne sont certainement pas sans valeur symbolique, les casques des conquérants romains paraissant tenir captif celui qui personnifie leur ennemi vaincu.

Ce vase est un produit du potier ('neivs Ateivs, d'Arezzo (Italie) dont l'officine a été découverte, il y a quelques années, dans cette ville de Toscane ${ }^{106}$. Son style est parfaitement

(103) Representation classique de ces unerrieres scythes : Von Botuver, op. cil., pl. I.XXXIX. Voir atussi A. Rır:, Jicl. des Anl. romaines el grecques, 1883, aux mots : Amazone (p. 2.1) el pella (p. 468).

(10.4) Ispreraxiler, Recucil, I, p. 31, fig. 6.

(10) Voir les casques de combattants figures sur les colonnes Trajanne et Aurelienne a Rome : casixar et linspot, Man. d'arch. romaine, II, p. 312.

(106) Nous tenons à remercier ici vivement .1 . le Professeur A. Srixico, de l'T niversite de Milan, spécialiste de la poterie arétine, pour les précieux renseignements qu'il a bien voulu nous donner sur le maitre-céramiste cxivs Atrivs, à qui il a attribuc la fabrication du vase de cavailon, ainsi que pour tous les détails concernant son ornementation. 
caractérisé sur notre vase, principalement par l'existence d'armes offensives et défensives en "remplissage " entre les différents groupes de personnages. I)'autre part, le thème de combats entre Amazones et Grecs est représenté fréquemment dans le matériel archéologique provenant des fouilles de son atelier. Enfin, on peut rapprocher certains éléments du décor, de lypes déjà connus et bien déterminés, par exemple le candélabre ${ }^{107}$ et l'autel ${ }^{108}$.

2. Petite écuelle basse ì bords obliques (f. Drag 31) dont le pied manque (fiq. 69 c), fragments de 2 autres semblables et d'un bord de bol f. Haltern 7 (fig. 69 a).

b) Sigillée du sud de la (iaule : fond de bol f. l)rag. 33, estampillé : FEs'TV' (fig. 69 b) ${ }^{109}$. potier de Lezoux, période Trajan-.Intonin.

(.) Vases à parois minces:

17 fragments de 3 bols en poterie sablée, terre jaunitre recouverte d'un vernis orangé à éclal métallique, criblée de petits grains de sable quartzeux adhérant aux parois qui fonl comme un revètement granuleux (époque Tibère-claude).

D. Poterie peinte de Lezoux : fragments de panse de grand vase à enduil blanchaitre, orné de. handes peintes en marron et de lignes tremblées (figr. $69 \mathrm{~g}$ ), el anse à 2 rainures verticales (fig. 69.9 h.

II. Polerie commune. Fragments d'ollae à rebord plat el incisions au col : jalle en terre blanchaitre à engobe gris fer. sorte de cuvelle a rebord horizonlal et fond plat lII. : 9.2 cm. diametre : $27.4 \mathrm{~cm}$.) (fig. $70 \mathrm{e})$.

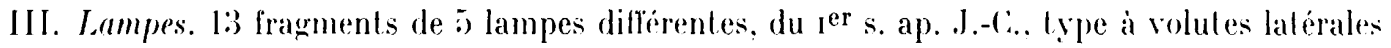
saillantes et ber probablement obtus, en argile jaune a vernis brun; fragment orné d'un casque (lig. 69) d e f).

20 Cocche noyswe: : aucun vestige archéologique;

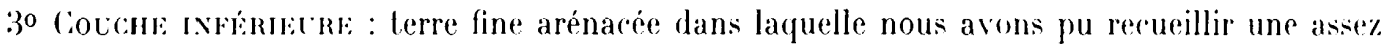
grande quantité de pépins dont la forme semble indiquer qu'ils appartiennent à des raisins du lỵpe Panse110. au milieu des vases brisés qui les avaient contenus ${ }^{111}$. Ce sont des urnes de la T'́ne 111 où se reconnaissent tous les types du puits $n^{0} 7$.

Forme $1: 1$ reconstiluée (fig. 70 a) et fragments d'une dizaine.

Forme $2: 1$ reconstituée (fig. 70 b) el 2 incomplites.

Forme $3: 1$ reconstituce avec 2 graflites à la base (fig. $70 \mathrm{~d}$ ).

(107) Représentation semblable dans A. Ox:, Arrelinische Reliefyefäse von Rhein, 1933, pl. XXXi, n० 135, pl. XL, n ${ }^{\circ} 142,144,145$, et dans A. STrico, Revisione crilica delle publicazioni sulla ceramica arrelina. I.isle di allri-

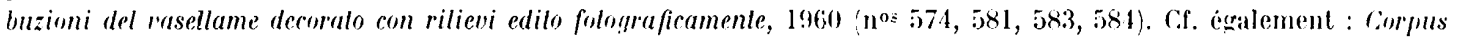
masorum anliquorum, L. S. A., Motropol. Museum of Art, New York, fasc. JCL. S. A. 9, pl. XXIX, 2 a et 2 h.

(108) A. Oxi, op. cil., pl. XXXII, XXXII, XXXIV, no 1:32, pl. XXXV, no 133. A. Strexico, op. cil., no: $n^{071}$

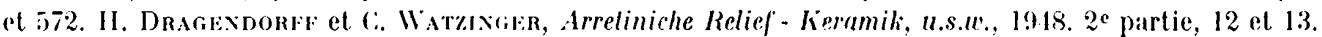

(109) Oswato, op. cil., p. 122.

110) Détermination de .I. le Professeur R. Salgues, de l'lustitut botanique de Brignoles (Var). à qui nous adressons nos vifs remerciements. Composition chimique de ces pripins :

pour 100 gr. de pépins, cendres 3,38

pour $100 \mathrm{gr}$. de cendres, chaux........ 31,52

silice.......... 21,20

(111) Columelle conseille, pour conserver les aliments, de les descendre dans des puits ou des citernes après les avoir enfermé dans des vaisseaux d'argile (cité par Punk, Hist. nal., XV, 18), qui ajoute (que ses contemporains mettent les fruits et raisins a conserver dans une fosse de 2 pieds de profondeur, garnie de sable. Les hommes néolithiques

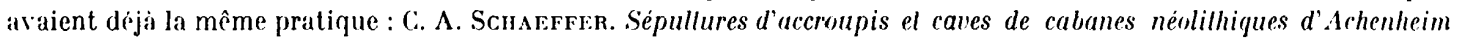
(Bas-Rhin), dans Cahiers d'Archeologie d'Alsace, 1926. 


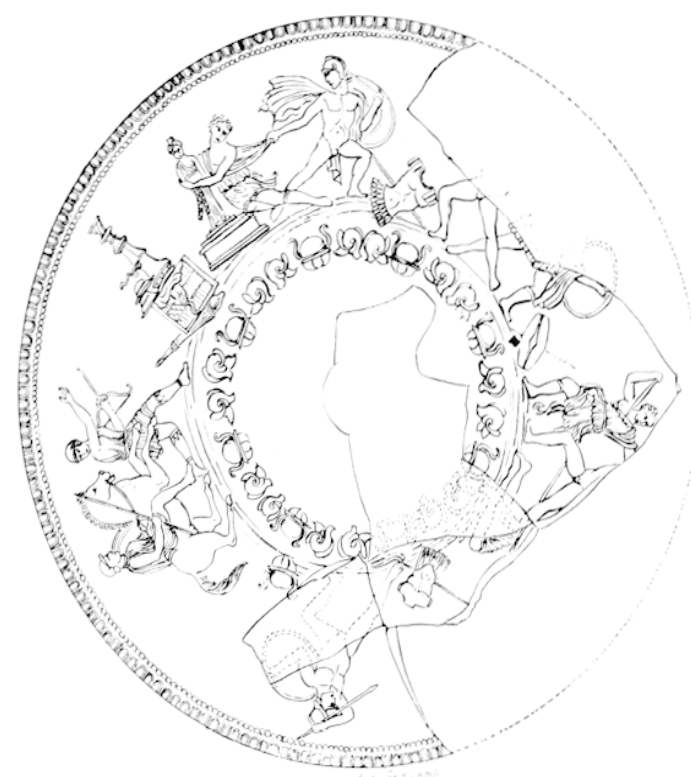

6x. Fosse no 1. Vase orne, develeppement du dicor.

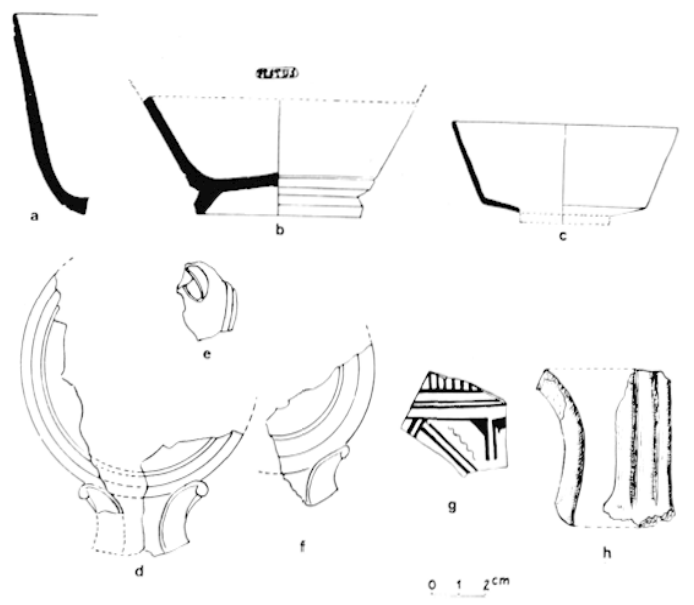

69. Fosse $n^{\circ}$ 1. Poterie sigillie, lampes, poterie peinte de l.e\%oux.
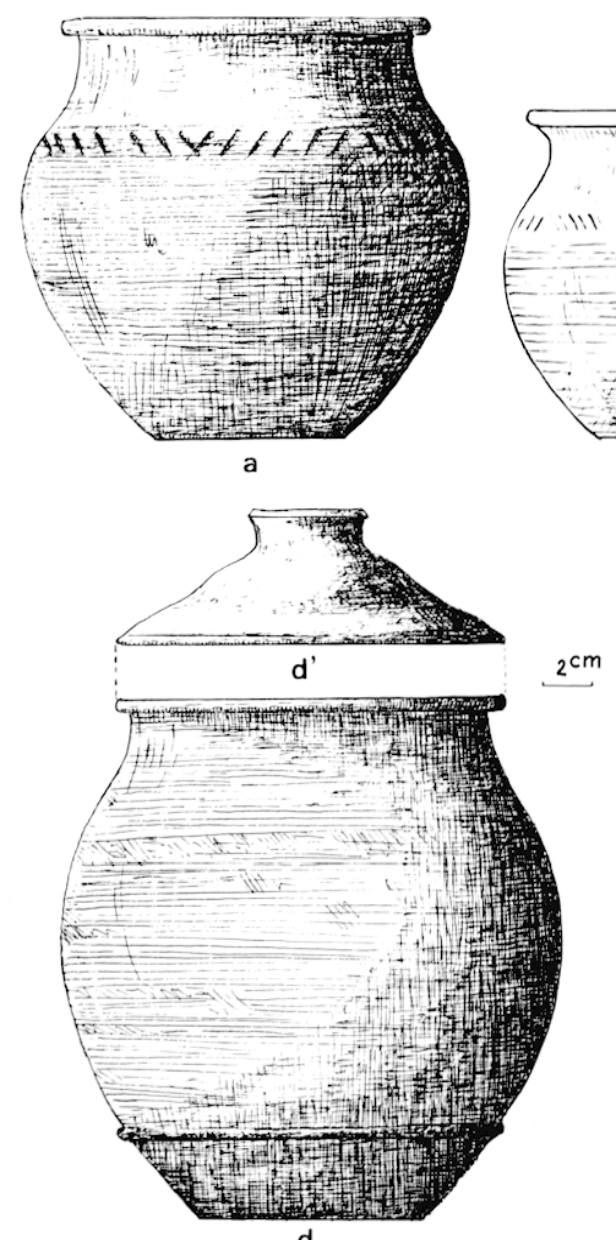

${ }^{\mathrm{cm}} \mathrm{cm} \mathbb{0}$
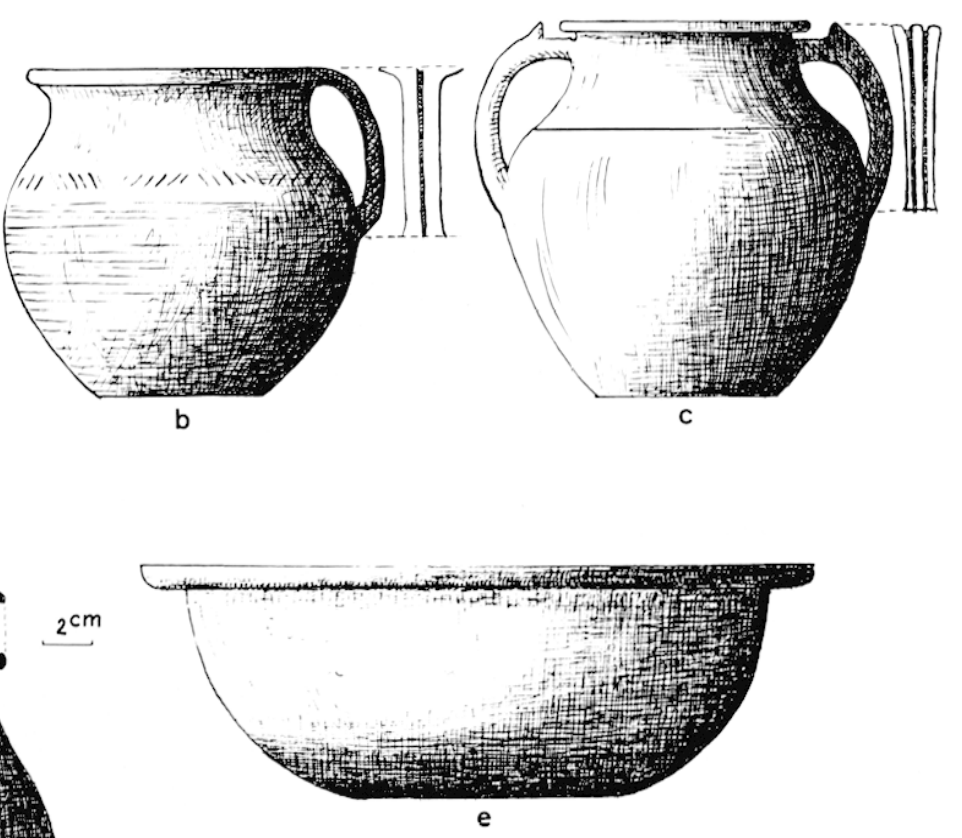

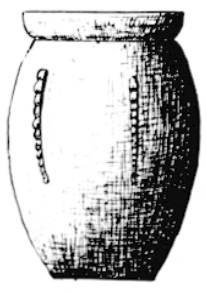

$f$

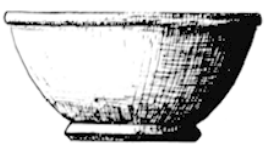

g

70. Fosse $n^{\circ} 1$. Poterie commune et vases à parois minces. 
Forme 4: 1 incompliele.

Forme 5: 1 reconstituée (fig. 70 c).

Courercles d'urnes : 1 seul entier et 4 fragments de 3 autres.

Il y avait encore : des fragments d'un plateau à pain, d'une jatte à goulol, d'une grande bassine $\dot{a}$ incisions sur le plat du bord, d'une jatte lustrée, de 2 vases a liquide en terre gris fer, d'une cruche en terre jaune (type a anse torsadée), un petit bol en terre jaune clair (f. Drag 7) (fig. 70 g). Enfin une petite urne à parois minces ornée de fins bourrelets d'argile cannelés appliqués verticalement sur la panse (fig. $70 \mathrm{f}$ ).

IV. Faune. ()s de brruf, mouton, pore.

La stratigraphie de cette fosse donne done, de bas en haut:

a) Une couche archéologique inférieure, de la $2^{\mathrm{e}}$ moitié du $\mathrm{I}^{\mathrm{er}}$ siècle av. .J.-(. correspondant à son utilisation de resserre pour conserver du raisin ;

b) Cne couche stérile indiquant l'abandon définitif du silo, suivie d'un remplissage, témoin de son utilisation comme dépotoir, surtout au début du ier siècle ap. .J.-li., aver prolongement jusqu'au ile (poterie sigillée de Lezoux).

FOSSE-SILO $x^{\circ} \stackrel{2}{2}$

Carrière Thiébold (octobre 1945). Peu profonde elle mesurait :3 m. jo de hauteur pour une largeur de $1 \mathrm{~m}$. I a couche archéologique très homogène, de la $2 \mathrm{e}$ moitié du I ${ }^{\mathrm{er}} \mathrm{s.} \mathrm{av.} \mathrm{J.-(C..,}$ occupait une épaisseur de $0 \mathrm{~m}$. 40 seulement à la partie inférieure de la fosse. Au-rlessus. sur $3 \mathrm{~m}$. 10 de hauteur, terre argilo-sableuse avec gros galets, stérile. Ici, l'abandon a éc définitif ; pas d'utilisation secondaire comme dépotoir.

I. Poterie fine. A. Prolo-campanien : fragment de bord de coupelle. ('ampanien $1:$ I lessons de vases. forme imprécise. Campanien $B$ : 3 fragments fond de bol, el pied de grand plateau aver. graffite en dessous (fig. 71 a). Campanien ( : : bords de 2 plateaux. Imitation de Campanien : 2 fragments de panses d'écuelles à reflets métalliques. el 1 petit fragment de fond en terre jaune à vernis brun rlair.

B. Italique : fragments de 2 bords d'assiettes f. Haltern 1 et Drag. 17 (fig. 71 b ( ) et 1 bord de bol f. Drag. 27 (fig. $71 \mathrm{~d}$ ).

C. Vases à parois minces : fond de petite urne concave en dessous, fragments de panse noir brillant, marron et rouge mat.

1). Poterie lustrée : fragments de pied et de paroi de 2 vases tronconiques trés évasés (forme du puits no 7 ) ayant pu servir de couvercle.

11. Poterie commune. A. Cirande urne à col court lisse, rebord étroit plat, panse peignée. avec graflite au col, paraissant indiquer une contenance (fig. 71 h) H. : $35 \mathrm{~cm}$. I). 0. : 20.3 ('m. I. 13. : $18 \mathrm{~cm}$. Olla incomplete (forme 3 du puits $\mathrm{n}^{\mathbf{0}} 7$ ) (6 fragments d'une olla forme I el 21 fragments de 4 autres. 2 fragments d'une urne lisse à anse (f. i), petit couvercle d'olla. entier.

B. Fragments de paroi peignée de grand bassine, de bord à grouttiere profonde de jatte à goulol, de pilhos avec bourrelet épais prìs de la base.

III. Poterie jaune. Vase à liquide à 2 anses arrondies rebord verlical plat sur le dessus pied horizontal (H. : 35,5 (cm. I). M. : $28 \mathrm{~cm}$.) (fig. $71 \mathrm{~g}$ ), fragments d'olpe (types du puits no 7 ) et 3 anses; 

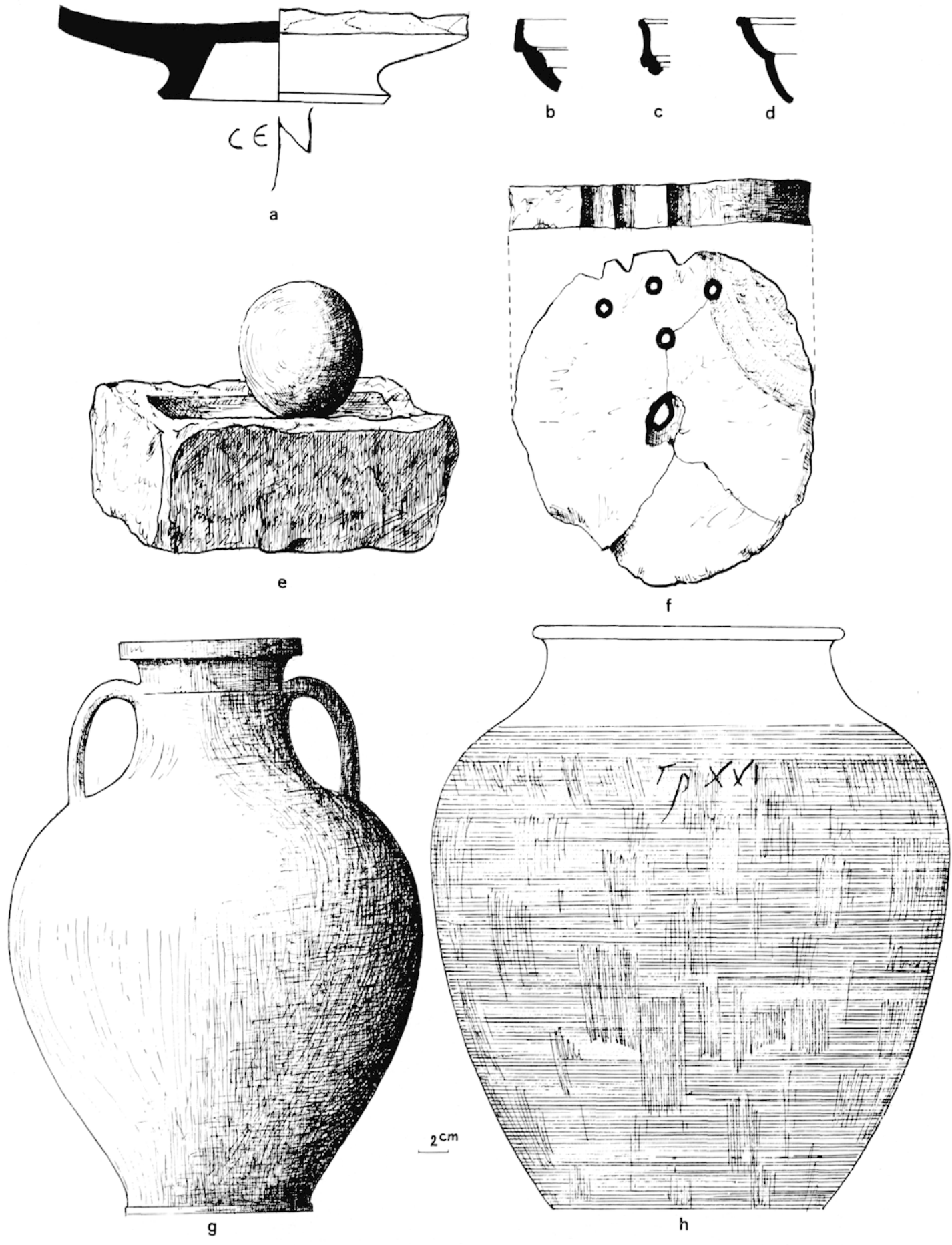

71. Fosse no 2. Céramiquue, meule et disque découpé dans une tuile plate. 
disque découpé dans une tegula, percé au centre et en 4 endroits près du bord, qui est entaillé sur la tranche de 3 encoches profondes (D. : $19 \mathrm{~cm}$. Ep. $3 \mathrm{~cm}, 2$ ) (fig. $71 \mathrm{f}$ ).

IV. Bronze. Fibule a charnière, de petite taille, ouverte (L. : $3 \mathrm{~cm}, 7$ ) (fig. 72 a), semblable à celle du puits $n^{0} 7$. Autre fibule à ressort bilatéral, incomplète (fig. $72 \mathrm{~b}$ ), grand anneau fermé à tige ronde (D. : $3 \mathrm{~cm}$ ) (fig. $72 \mathrm{~d}$ ) petit disque plat percé au centre (fig. $72 \mathrm{c}$ ), bracclct incomplet à tige lisse (fig. $72 \mathrm{e}$ ).

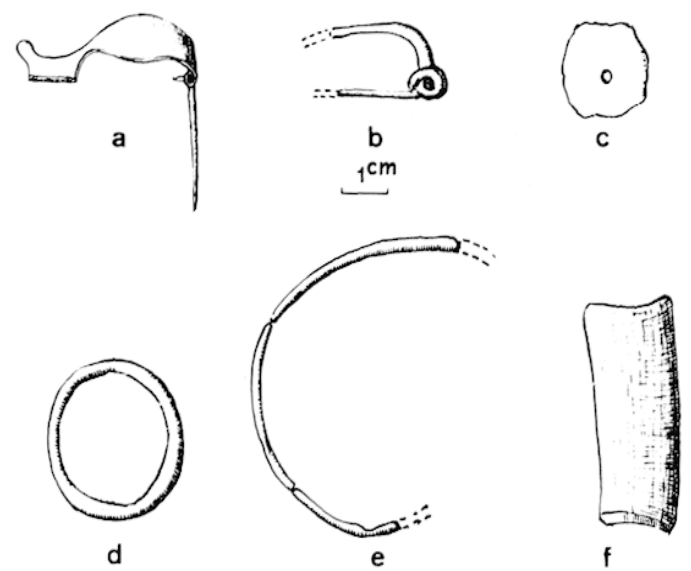

72. Fosse no 2. Objets en bronze et en os.

V. Divers. 9 clous en fer; moitiè de manche de préhension en os d'un petit outil en fer (peut être une alène) (fig. $72 \mathrm{f}$ ); fragment de récipient en verre à base angulaire ; petite meule rectangulaire en mollasse, à va-et-vient, avec cuvette d'utilisation très marquée et broyeur sphérique (fig. 71 e), 5 fragments de plaques de revêtement en argile.

VI. Vestiges alimentaires. La couche de base sableuse contenail des pépins de raisins semblables à ceux de la fosse $n^{0} 1$ ( $1 / 2$ kilo), des fragments d'une galette de céréales, des pois chiches (cicer ariétinum L.) des os de poisson, mouton, bruf et porc.

FOSSE $N^{\circ} \quad 3$

Découverte en novembre 1948, face au puits $\mathrm{n}^{0} 7$ sur le front ouest de la carrière Thiébold, elle avait une hauteur totale de $8 \mathrm{~m}$. et une section horizontale carrée de $1,50 \mathrm{~m}$. de còté (fig. 73). La partie supérieure s'était éboulée sur toute la hauteur du front de carrière. soit $5 \mathrm{~m}$. environ. Parmi des décombres : enduits muraux, galets, socle en mollasse, ont été recueillis les objets suivants :

1. Monnaie. Dupondius de Tibère (14-37 ap. J.-(i.). Avers : TI. CAEsiLR. DIVI .AVG. F. AVGVsT. IMP. VIII. Tête laurée de l'empereur à gauche. - Revers : PONTIF. M.IX. TRIBVN. POTEST. XXXIIX. Globe auquel est attaché un gouvernail, entre s' et C (fig. 74 a). Pièce frappée en 36 ap. J.-C. ${ }^{112}$.

II. Céramique. 1. Grand vase à 2 anses en poterie commune (cf. puits no 7 , fig. 27 a), (II. : $27,4 \mathrm{~cm}$. D. M. : $27 \mathrm{~cm}$.). Moitié de couronne en terre cuite rougeâtre.

2. Lampe en argile jaune à vernis marron peu adhérent, cuvette creuse, avec trou central entouré de 2 cercles concentriques limitant un léger bourrelet circulaire, ornée d'une couronne de feuilles cordiformes sans pétiolc, volutes latérales saillantes et enroulées, bec en arc de cercle (f. Dressel 9, variante) (fig. $74 \mathrm{~b})^{113}$.

3. Fragments de lampes ornées : arrière-train de sanglier à gauche (fig. $74 \mathrm{c})^{114}$. Victoire de face (fig. 74 d), bec de lampe dit à «têtes d'oiseaux» (f. 34 d'Haltern, 4 de Dressel) (fig. 74 e) e $^{115}$.

(112) Cones, I, p. 191, nº 12, 3e variété.

(113) Décor similaire sur une lampe figurée par II. B. Walters, op. cil., figr. $131, n^{\circ} 736$ (rer s. ap. J.-C.).

(114) Fragment identique au Musée de Glanum : P. de Brts, op. cit., p. 19, pl. VI, no 6.

(115) Ces lampes sont considérées comme caractéristiques de l'époque augustéenne, disparaissant sous Tibère : Labrotsse, op. cil., fig. 1, p. 15. Mr.xze, Calalogue des lampes dit Musie de .Mayence, 1956. 

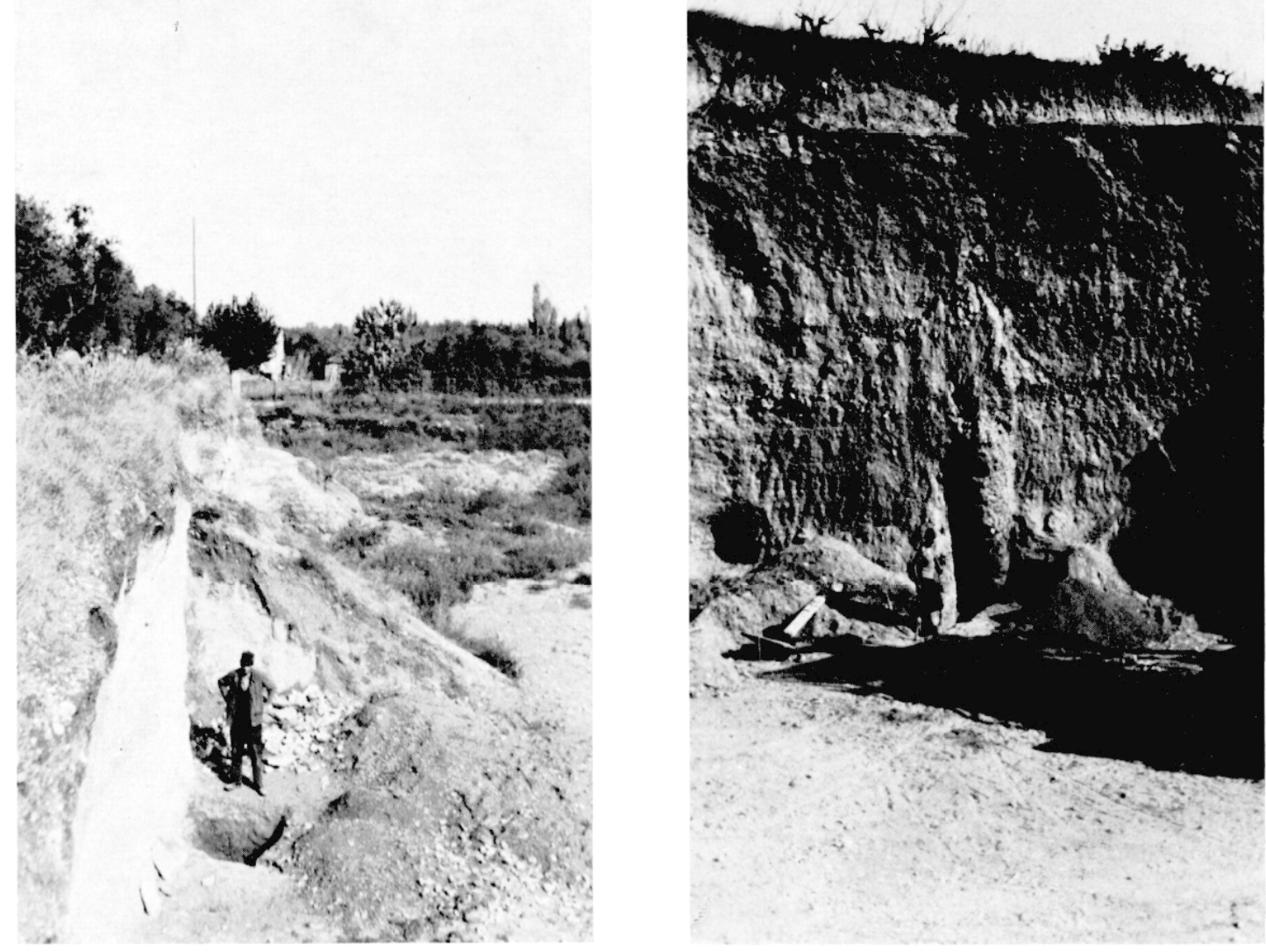

73. A gauche, fosse-silo $n^{\circ} 3$; i droite, puits $n^{\circ} 10$.
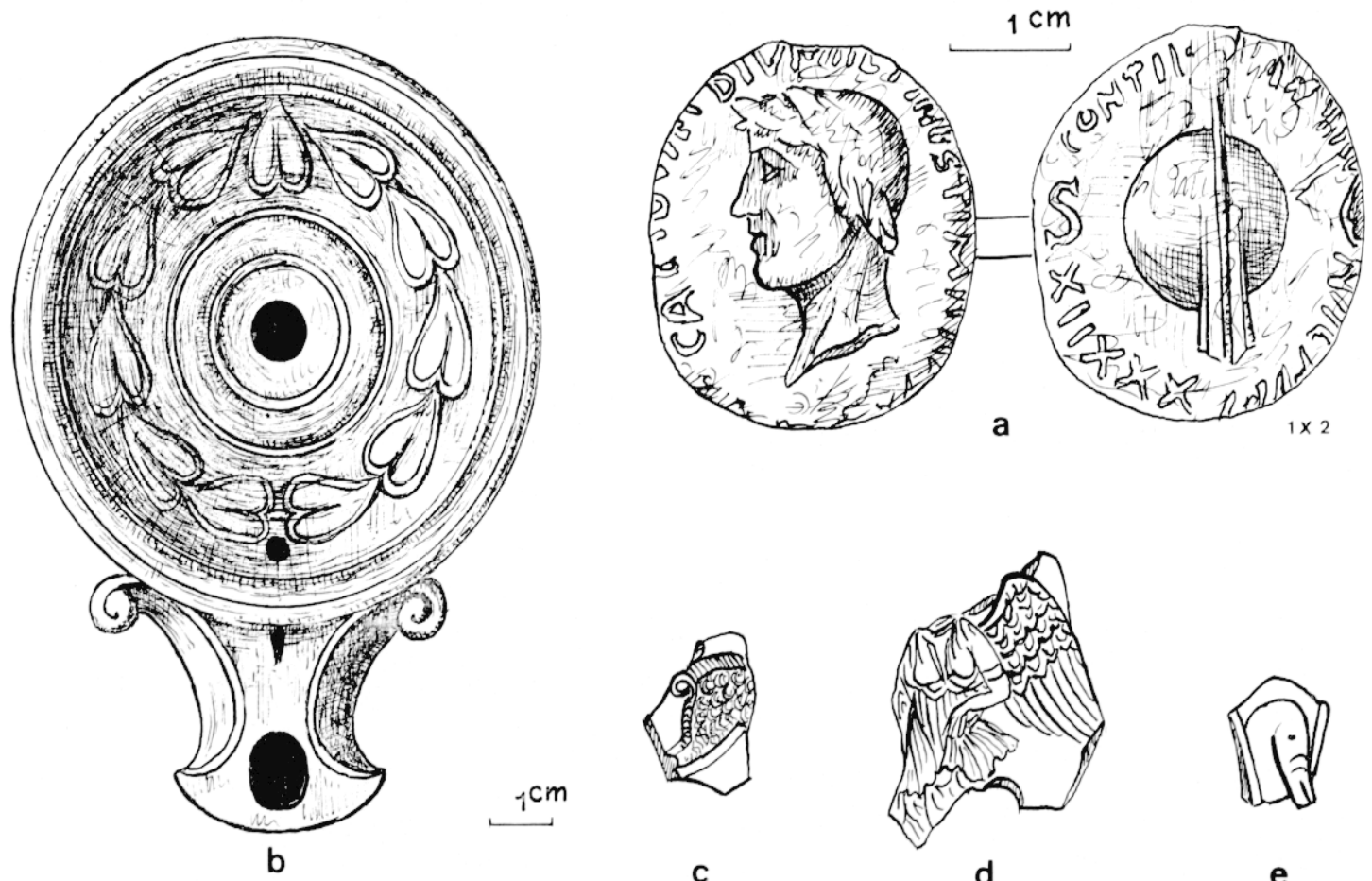

74. Fosse-silo no 3 . Dupondius de Tibère, lampe et fragments de lampes ornées (couche supérieure). 
Dans les 3 mitres en dessous, la terre était friable, cendreuse par endroits et plus riche en vestiges archéologiques, accompagnant des restes alimentaires.

1. Monnaies. $1^{0}$ Petit bronze de Massalia, assez fruste : Ivers : lète d'Ipollon lauré, à droite.

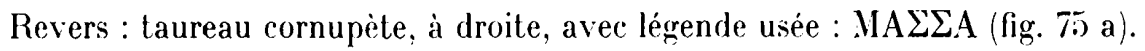

$2^{n}$. Petit bronzc des Volques Arćcomiques, du même type que celui découvert dans le puils no 7 (fig. $75 \mathrm{~b}$ ).

II. Objets métalliques. Petite boucle à 3 trous (fig. 76 a), tige bifide (fig. 76 b), clou à tête ronde (fig. 76 c), 2 plaquettes et 5 fragments en bronze. Clous et plaques de fer ; fragment de plomb informe.

III. Céramique fine. A. Bucchero gris massaliote, de Villard : fragment de bord de vase à traits: ondés (fig. 76 e), autre bord allongé à engobe gris foncé très adhérent et tesson épais en argile tendre.

B. Campanien $\perp$ : fragment de rebord de patère à marli (fig. $76 \mathrm{~h}$ ) et de fond à bandes peintes en clair (fig. $76 \mathrm{f}$ ).

C. Imitation régionale des formes campaniennes : 1. Argoile rougeâtre à vernis marron plus ou moins foncé. C'est la forme écuelle, genre "saladier», qui domine (fig. 77 a el 85 P et T), comme dans le puits $n^{\circ} 7.3$ ont été reconstituées (l'une porte le nom de son possesseur gravé sous le pied (fig. 77 c). 2 sont incomplètes, une centaine de tessons (dont l'un à bande peinte fig. $76 \mathrm{~g}$ ) et 3 avec graffiti (fig. $77 \mathrm{f} \mathrm{g} \mathrm{h}$ ), se rapportent à 7 écuelles de même type.

2. Argile jaune, dure, à vernis brun brillant (peut-être type $\mathrm{F}$ de Lamboglia). Écuelle à panse oblique légérement coudée vers le bas, à pied creux profond (fig. 77 b et $85 \mathrm{R}$ ) et 2 autres incomplètes.

I). Campanien C : fragments de bols, f. 1, à double sillon au bord, et f. 19 à bord déversé. 2 fragments d'un grand plateau et fragment de pied d'un autre, avec stries circulaires ; pied d'écuelle avec graflite répété (fig. $77 \mathrm{i}$ j).

E. Imitation de Campanien $C$ : petit bol entier à rebord évasé avec nom de possesseur à la partie inférieure du pied (fig. $77 \mathrm{~d}, 78$ et 85 G), 1 plateau incomplet à surface usée et fragments de 2 autres.

F. Pré-sigillée : coupelle à pied large, en terre jaune à vernis brun brillant (H.: $3 \mathrm{~cm}$. I). (). : $8 \mathrm{~cm}$.) (figr. 77 e et $85 \mathrm{~A}$ ) et fragments d'écuelle à bord courbe et sillon près du bord, absolument identique à un fragment du puits $n^{0} 11$ (fig. 58 b).

G. Sigillée italique : $1^{\circ}$ Vase orné dont il manque le pied et toute la partie supérieure (fig. 79). Le décor représente un rinceau d'éléments végétaux composé chacun d'une tige renfléc fibreuse verticale naissant et se terminant par une corolle monopétale en entonnoir ; de sa base partent, latéralement et symétriquement, 2 tortillons et 2 longues feuilles d'acanthe à pétiole gainé. La soudure du rinceau est formée par une palmette sur une grande feuille de lierre avec tortillons pendant et enroulement latéraux aboutissant à une fleur à 4 pélales triangulaires ou à 14 globules.

Au-dessus, séparée par une rangée de perles, frise d'armes défensives. De g. à dr. : pella lunata (bouclier des Amazones), casque grec à longue visière et cimier à crinière flottante, de profil ; 2 boucliers disposés en éventail, l'un ovale, l'autre ovale-tronqué ; autre casque rond, à visière triangulaire et paragnathides, vu de face. Ensemble qui se répète 3 fois.

Il s'agit certainement d'un produit arétin mais étant trop incomplet on ne peut préciser 

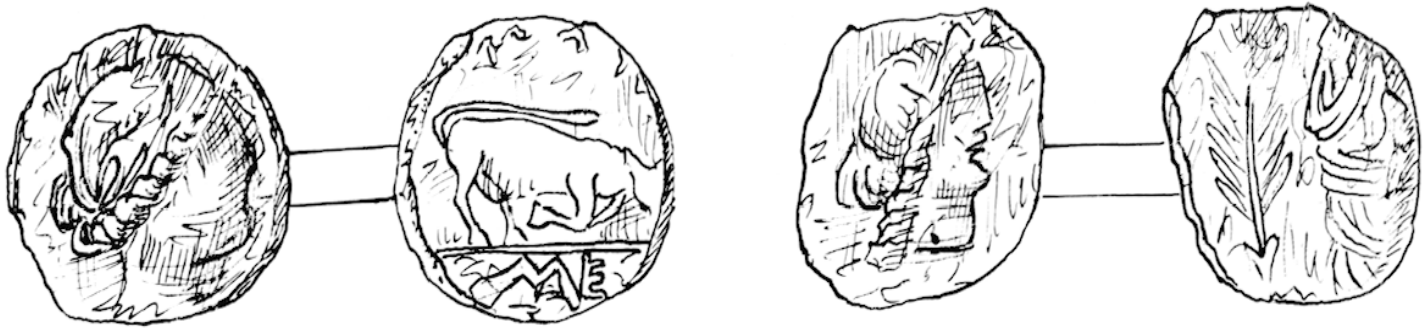

a

$1^{\mathrm{cm}}$

b

75. Fosse-silo n 3. Petits bronzes de Massalia et des Volques Arécomiques (couche inférieure).

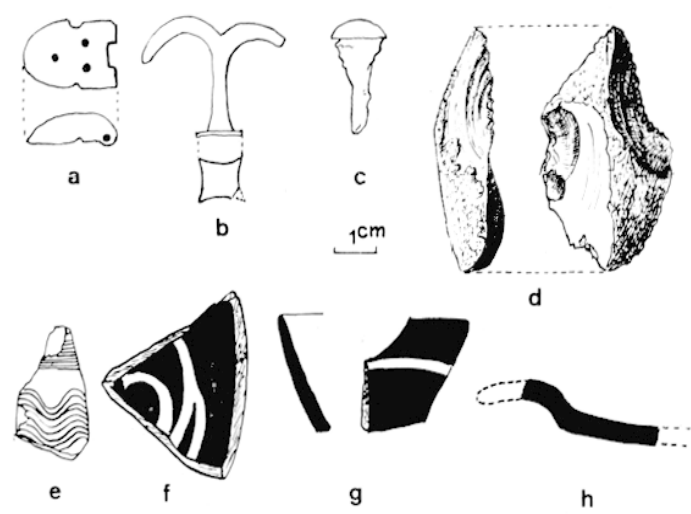

76. Fosse-silo no 3. Ohjets en bronze, silex taille, poterie phocienne et campanienne.

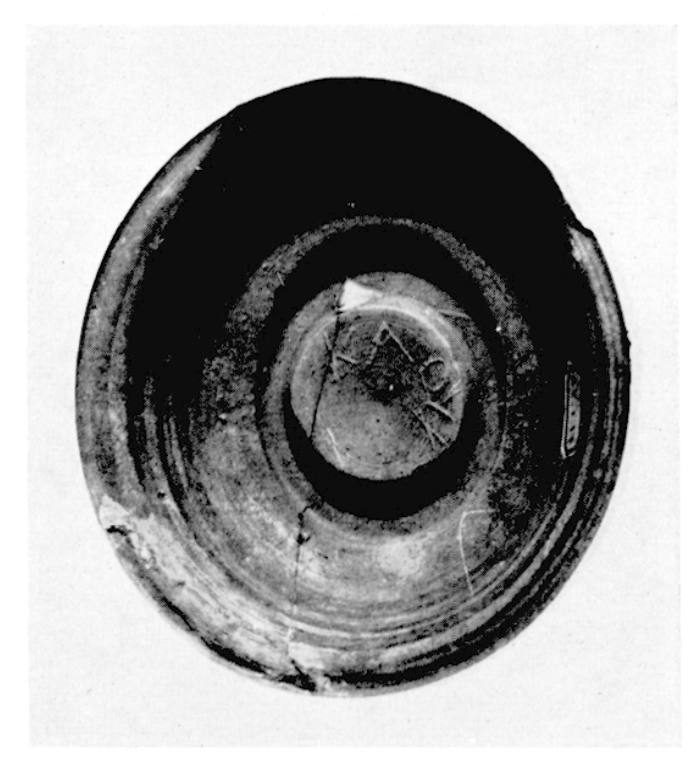

7x. Fosse-silo no 3. Bol en poterie d'imitation de campanien $C_{\text {, }}$ avec nom du possesseur du vase en caractires grecs: KAOYA.

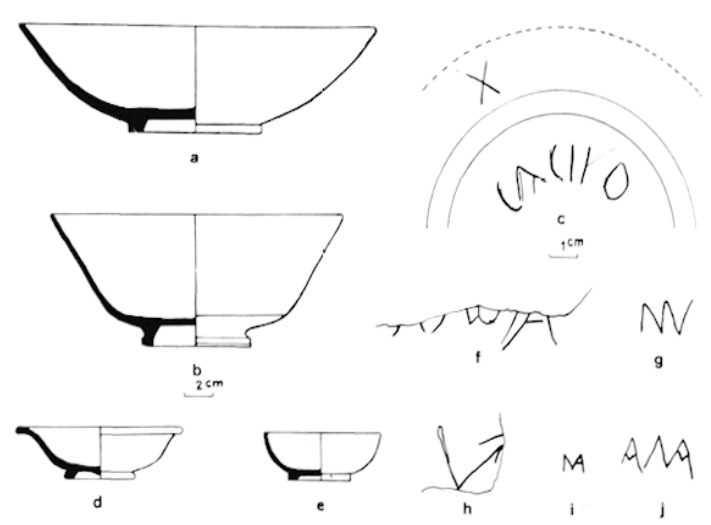

77. Fosse-silo no 3 . Vases d'imitation de campanien, pré-sigrillée et grafrites.
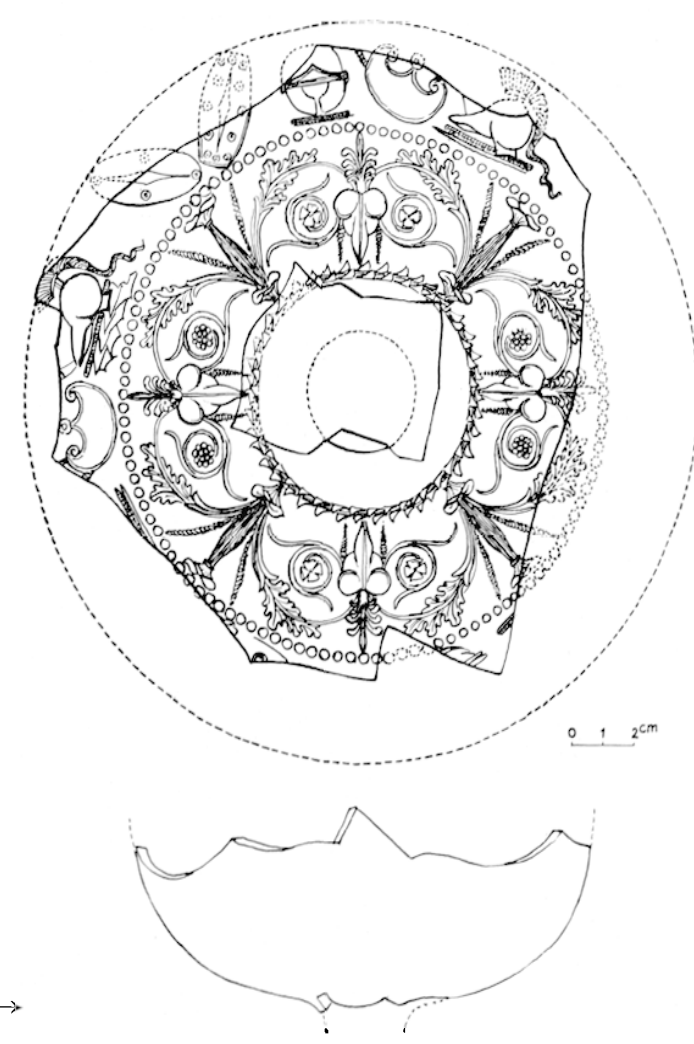

79. Fosse-silo $n^{\circ}$ 3. Fond de vase arétin orné. $\rightarrow$ 
l'officine d'où il est issu. Tout au plus peut-on faire quelques comparaisons avec des vases bien déterminés où figurent certains éléments semblables du décor. Par exemple, du potier L. Titius Thyrsus, dont on connaît un vase signé au Musée de New York, on peut rapprocher la feuille d'acanthe à gaine et la fleur à globules ${ }^{116}$. De L. Avillius Sura, les boucliers du trophée du vase de ce potier, figurés par $0 x e^{117}$, sont semblables aux nôtres, ainsi que les petits triangles alternant sur double rangée à la base du décor, d'un fragment de moule, et d'un tesson du Musée d'Arezzo ${ }^{118}$. Dans la production du potier Publius, sur certains tessons et moules figurés par A. Sténico, on retrouve le bouclier ovale $e^{119}$ et le casque rond vu de face ${ }^{120}$. Ce même casque est aussi représenté chez C. Cispius ${ }^{121}$ dont les enroulements, végétaux sont également très proches de ceux du vase de Cavaillon ${ }^{122}$;

$2^{0}$ Vases lisses. a) Assiette, forme Haltern I, marquée : ATEI (A et T liés) (service 1 de l'atelier d'Arezzo, 30 à 10 av. J.-C.. ${ }^{123}$. Sous le pied, graffite à la pointe : MOY (H. : $2 \mathrm{~cm} .5$ D. $0 .: 18 \mathrm{~cm} .2$ ) (fig. 80 e et $85 \mathrm{~F}$ ) et 2 autres fragments de type semblable.

b) Bol f. Haltern 7, estampillé C. S. F. (H. : 7 cm. 4 - D. 0. : 14 cm.) (fig. 80 a et 85 E) (Arezzo, service 1);

c) Petit bol, f. Ritt 5 A, signé ATEI (H. $4 \mathrm{~cm} .5-$ D. $0 .: 7 \mathrm{~cm} .5$ ) (fig. 80 b et 85 (i) (Arezzo, service II, 20 av. J.-C. - 10 après) et 1 bord d'un autre identique.

d) Bol même forme, avec estampille en 5 lignes dans un cercle : FECIT MALIVS FORTVNATVGI, sous le pied en graffite : PI (H. : $5 \mathrm{~cm} .5 \mathrm{D} .0 .9 \mathrm{~cm} .4$ (fig. 80 c et $85 \mathrm{D})$;

e) Bol bas (f. Drag. 31) marqué ABBI ; sous le pied, graffite : HOY, comme sur l'assiette décrite plus haut (H.: $4 \mathrm{~cm}$. D. $0 .: 11 \mathrm{~cm}$.) (fig. 80 d et $85 \mathrm{~B}$ ) ; 1 autre, sans pied, 2 fragments de bord et une moitié de fond même type.

f) Fragments d'assiettes et de grand plateau à bandes circulaires intérieures hachurées (f. Drag 15/17), de bord f. Drag. 27, de panse guillochée de tasse.

H. Vases à parois minces : gobelet caliciforme à pied creux, étroit (H.: $8 \mathrm{~cm}, 5$. D. $0 .: 10,6 \mathrm{~cm}$.) (fig. $80 \mathrm{f}$ et $85 \mathrm{I}$ ), bol à pied bas, en argile bleuâtre à la cassure et engobe marron mat (fig. $80 \mathrm{~g}$ ), gobelet à lèvre en bourrelet extérieur et fond concave (H.: $9 \mathrm{~cm}$. D. $0 .: 10,2 \mathrm{~cm}$.) (fig. $80 \mathrm{~h}$ et 850 ), gobelet tronconique à parois extrémement minces (moins de $1 \mathrm{~mm}$ ), dont la base manque (fig. $80 \mathrm{i}$ ), fragments de différents petits vases lisses, ou ornés à la barbotine, de bâtonnets, ou triangles (formes du puits $\mathrm{n}^{0} 7$ ), fragment de base de gobelet du potier Aco avec décor caractéristique (fig. $80 \mathrm{k}$ ), fragment de coupe à surface sablée et d'unguentarium.

I. Poterie à enduit rouge : a) Enduit très adhérent intérieur, argile fine homogène : fragment de bord de jatte carénée, identique à celle du puits no 7 .

(116) Corpus vasorum antiquorum, op. cit., pl. XXXIII, 1 a et 1 b. A. Stevico, op. cil., no 851.

(117) Oxí, op. cit., pl. LII, no 220 a.

(118) A. Strenico, Nuovi frammenti di L. Aviluis Sura nel Museo archeologico di Arezzo, dans Bull. della 'omm. arch. Com. di Roma, LXXV (1953-55) (p. 24 fig. 3 et 4).

(119) A. Stricico, Sulla produzione di vasi con rilievi di C. Cispius, dans Alhenaeum, XXXIi, 1955, pl. 11.

(120) Id., ibid., pl. II.

(121) Id., ibid., pl. II, no 20.

(122) Id., ibid., pl. IV, no 42. Tav. I no 4, 7, 14.

(123) N. Lamboglia, Apunles sobre cronologia ceramica, p. 80 et pl. VI. 


\section{(CSIPi}

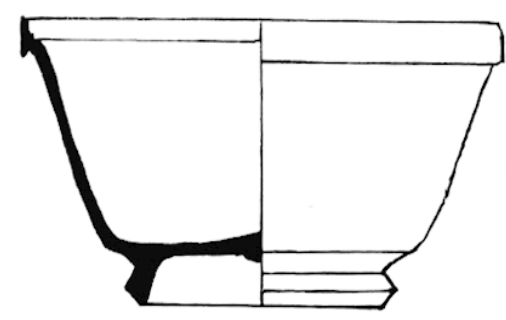

a

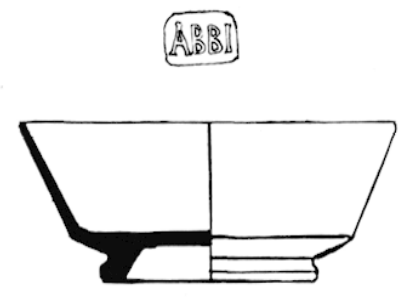

$M \supset Y 1^{\mathrm{cm}}$

d
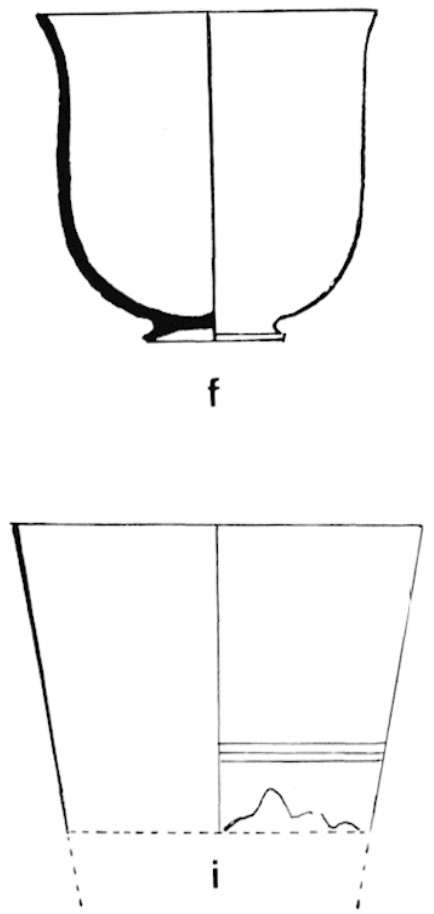

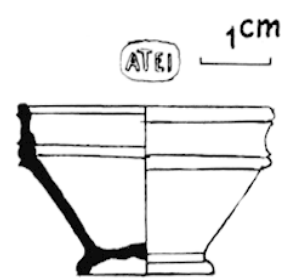

b

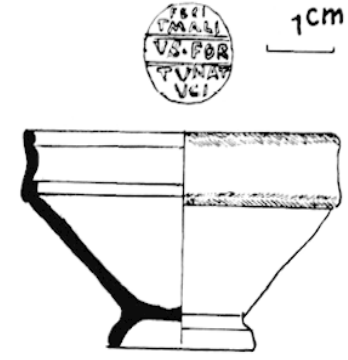

f)

C
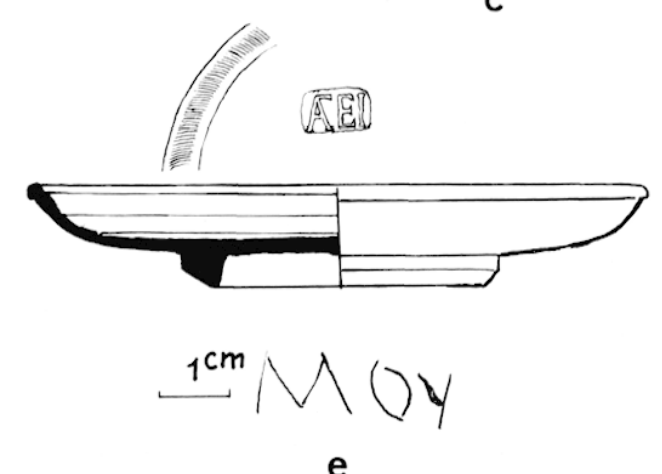

e
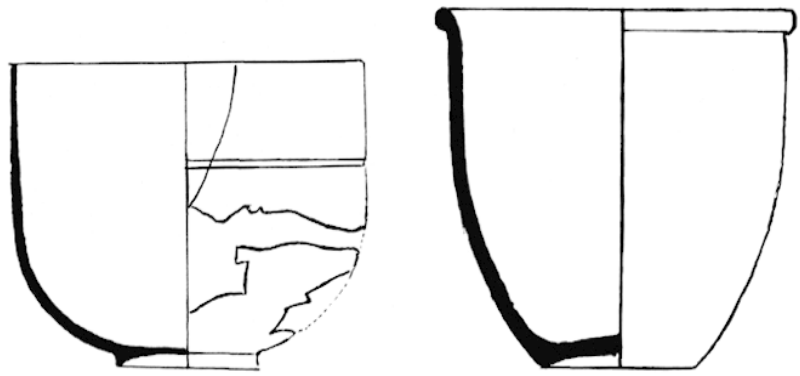

g

h
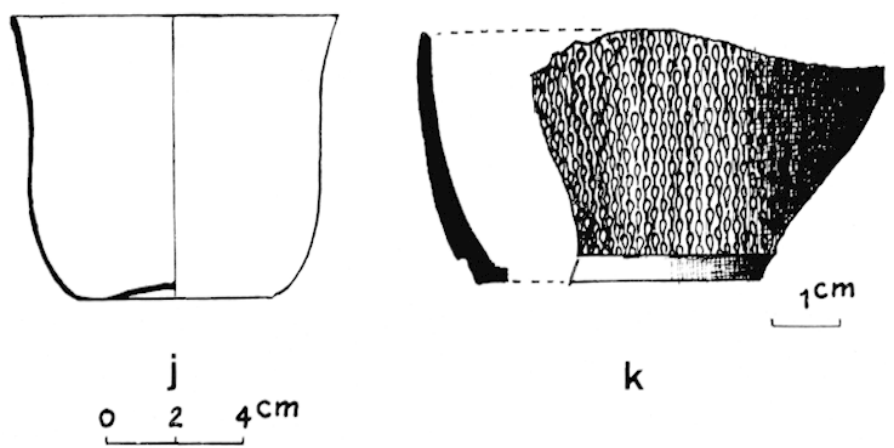

k

80. Fosse-silo $n^{\circ} 3$. Poterie sigillée arétine, à parois minces et gobelet en verre. 
b) Enduil peu adhérent intérieur, argile granuleuse : fragment de bord de jatte arrondie, a levre plate, et 6 fragments d'une pelite urne à bandes horizontales plus fonc'ées.

J. Poterie à décor oculé : coupe semblable à celle du puits no 7 , ornée de 2 rangées d' " yeux" superposées (H. : 4,8 cm. D. $0 .: 13,5 \mathrm{~cm}$, ) (fig. 81 et $85 \mathrm{~L}$ ), fragment d'une coupe plus grande, avec 6 rangs de ce décor caractéristique.

K. Poterie peinte de Lezoux (?): bol peu élevé à enduit blanchàtre, panse convexe, le fond manque (fig. $82 \mathrm{~d}$ ). Fragment de vase caréné et de coupe à bord plat, peint uniformément en blanc laiteux.

L. Poterie lustrée : a) Argile marron à engobe foncé : fragments de 33 écuelles à pied creux.

b) Argile rose à engobe de mème couleur : 3 assiettes reconstituées de taille différente mais de profil identique, provenant d'un même service (Diamètres : $13,8 \mathrm{~cm} .-19, \overline{5} \mathrm{~cm} .-233, \mathrm{~cm}$.) (fig. $82 \mathrm{c}$ ).

c) 2 autres assiettes incomplètes, dont une à bord noirci, et un fragment de bol.

IV. Céramique commune. A. Crnes : toutes du type de la Tène III, où se retrouvent les formes du puits $n^{0} 7$.

Forme 1: 1 intacte, qui se trouvait dans la position renversée, tout i fait, au fond de la fosse (fig. 85) $\mathrm{M}$ ), 1 reconstituée (fig. $85 \mathrm{~S}$ ) et 17 incomplètes.

Forme $2: 1$ entière (fig. 85) $Q$ ).

Forme 3: 50 fragments représentant 3 urnes.

Forme 4: Urne micacée à panse rugueuse, incomplète.

Forme 5 (urnes lisses) : 2 entières (l'une a les 2 anses terminées sur la panse par 3 digitations rappelant les attaches d'anses des vases en bronze (fig. $82 \mathrm{~b}$ et $85 \mathrm{~K}$ ), 1 incomplete et 18 tessons.

B. Couvercles d'ollae. 1 entier (fig. $85 \mathrm{M}$ ), 3 incomplets, et 36 fragments de 4 autres.

(.. Jattes à goulot : fragment de panse à anse en ruban, caractéristique, et 2 fragments de bord.

D. Plateaux à pain : fragment de bord à 3 languettes circulaires appliquées et a fragments de 3 plateaux différents.

E. Marmites : 1 fond et 27 fragments de 3 récipients identiques.

F. Bassine : 4 fragments de bord, 3 anses et 23 fragments de panse peignée (2 récipients).

V. Poterie jaune. a) Vase à liquide à col cylindrique large et élevé. panse galbée, pied en bourrelet, anse courbe, allongée, à 2 sillons longitudinaux (H. : $28,2 \mathrm{~cm}$. I. .11. : $21 \mathrm{~cm}$.) (fig. 82 e e)

b) Vase à liquide à 2 anses arrondies simples, col cylindrique un peu évasé vers le haut, sans rebord, panse ovoide (H. : $25 \mathrm{~cm}$. D. M. : $20,4 \mathrm{~cm}$.) (fig. $82 \mathrm{f}$ ).

c) Jatte incomplète en forme de calotte sphérique pédiculée, avec graflite sous le pied (fiq. \$2 a).

d) Inse torsadée, et fragments de 2 vases, forme 1 du puits $n^{0} 7$, dont un col avec graffite : ЕСК ІГГАI (E et Ci lunaires) ${ }^{125}$ (fig. 8:3).

e) Mortier incomplet avec emplacement pour la préhension.

f) Couronne en terre cuite de forme habituelle.

(124) Forme de vase rencontrée dans les tombes gallo-grecques de Saint-Rémy-de-Provence : P. de Bri' np. cil., pl. 2, fig. 10.

(125) Н.CKГГГAI (d'Escinga), nom féminin déjà connu dans l'épigraphie celto-grecque régrionale par une inscription trouvée au pied de la colline de Perréal près d'Apt, à une trentaine de $\mathrm{km}$. de cavaillon : F. SAlvi, Lat région aplésienne, Éludes d'hisloire et d'archéologie, II, Gargas dans Mém. de l'Acad. de Vaucluse, 1905, p. 75, et aussi C.I.L., XII, p. 137, n² 2 . 

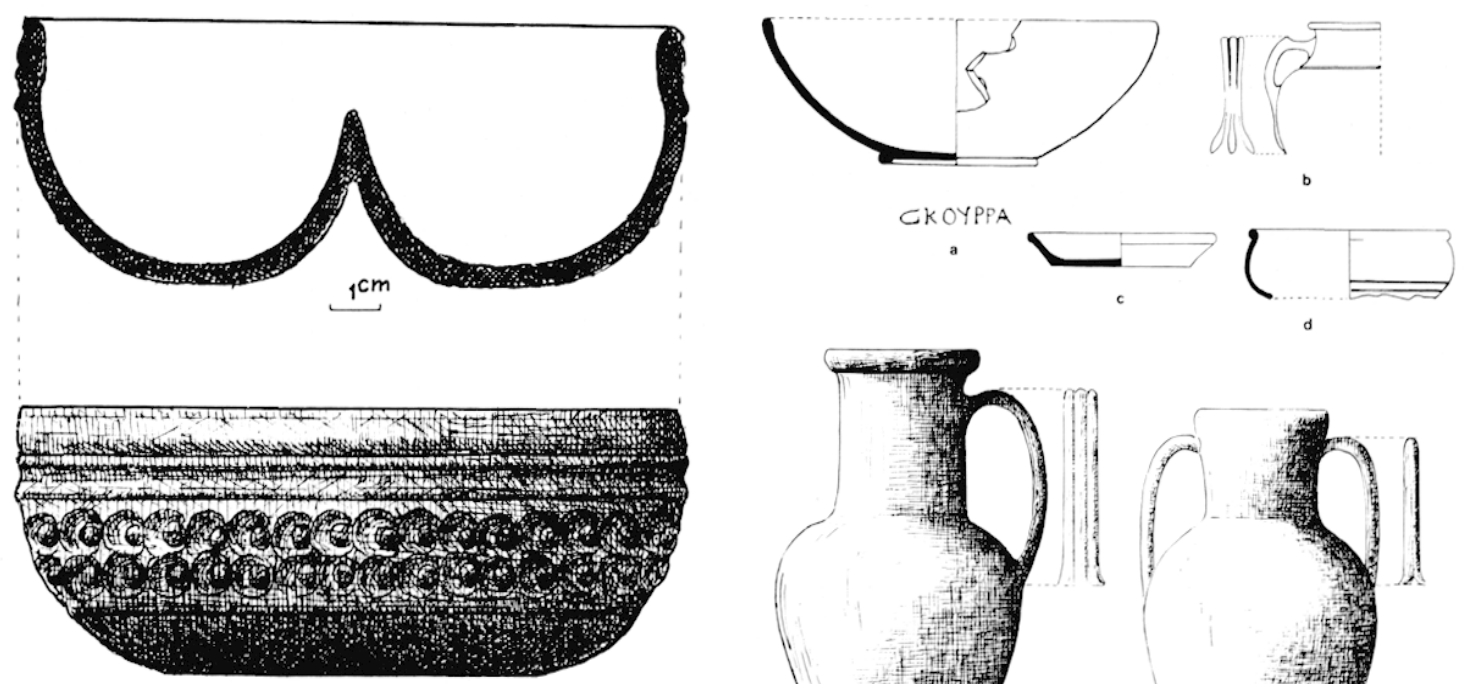

81. Vosse-silo $\mathrm{n}^{\circ} 3$. Vase à décor oculé et fond saillant intérieurement.
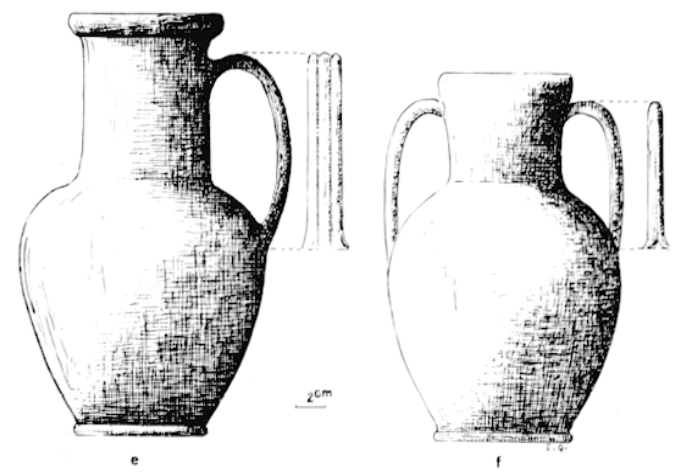

82. Fosse-silo no 3. Céramique jaune, urne à anse, jatte, bol.
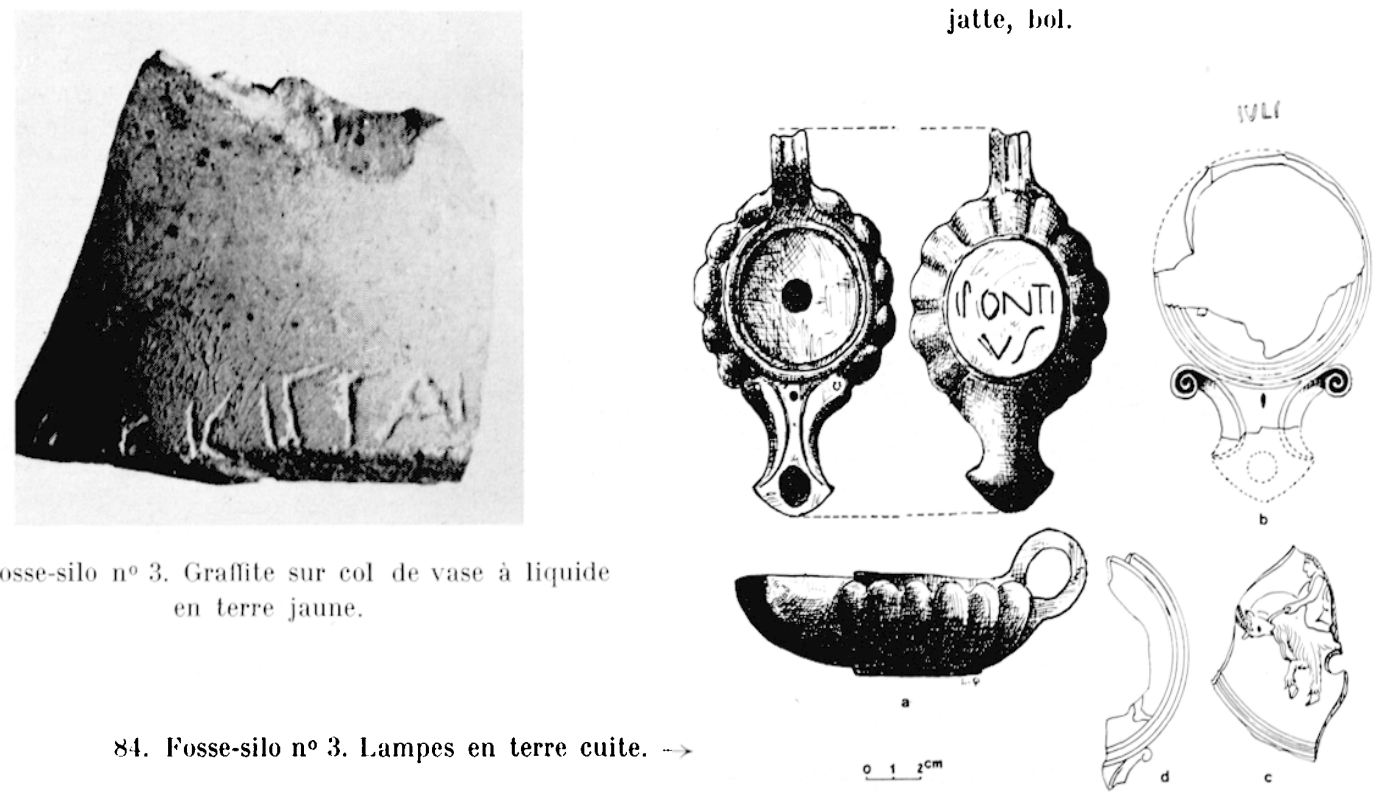

ช4. Fosse-silo no 3 . Lampes en terre cuite. $\rightarrow$ Graflite sur co
en terre jaune.

83. Fosse-silo $n^{\circ}$ 3. Graflite sur col de vase à liquide

VI. Poterie gris /er. Goulots de 2 vases à liquide, à bec tréflé, et une cinquantaine de tessons.

VII. Lampes. a) Lampe en argile jaune dure, à couverte brun rouge, réservoir godronné verticalement tout autour; trou central et anse rubanée; bec allongé, avec sur le dessus, ébauche de volutes, terminé en angle obtus. Marque sous le réservoir PONTIVs'se en 2 lignes, gravée à la pointe avant cuisson (type de lampe apparenté à la forme 10 de Dressel) (fig. 84 a et $85 \mathrm{~N}$ ) que l'on peut classer au début de l'époque augustéenne.

b) Lampe en terre jaune recouverte d'un vernis marron, volutes latérales saillantes et très

(126) R. BailLy, op. cil., p. 114 et fig. 115 , no 10 . Ce nom de fabricant de lampe ne se trouve dans aucune liste régionale. Dans Waitras, op. cil., une seule lampe, f. Dressel 9, porte ce nom sous la forme : L. PoNtı (p. 84, no jãl). 
enroulées, le dessus manque ainsi que l'extrémité du bec. sous le pied marque en relief : IVLI (fig. $84 \mathrm{~b})$.

c) Fragment de dessus de lampe orné : silène à cheval sur un bouc, à gauche (fig. $84 \mathrm{c}$ ).

d) Fragment droit de lampe a volutes, avec jambe humaine (fig. 84 d) et 7 petits fragments d'une autre lampe a couverte brun clair, dont un bec incomplet angulaire.

VIII. Verre. a) Gobelet en verre violacé épaissi vers le haut (variante de la f. 71 de Morin-Jean) ${ }^{127}$ (fig. $80 \mathrm{j}$ et $85 \mathrm{H}$ ).

b) Autre gobelet de même type, incomplet, ainsi qu'un petit fragment de récipient à décor alvéolaire.

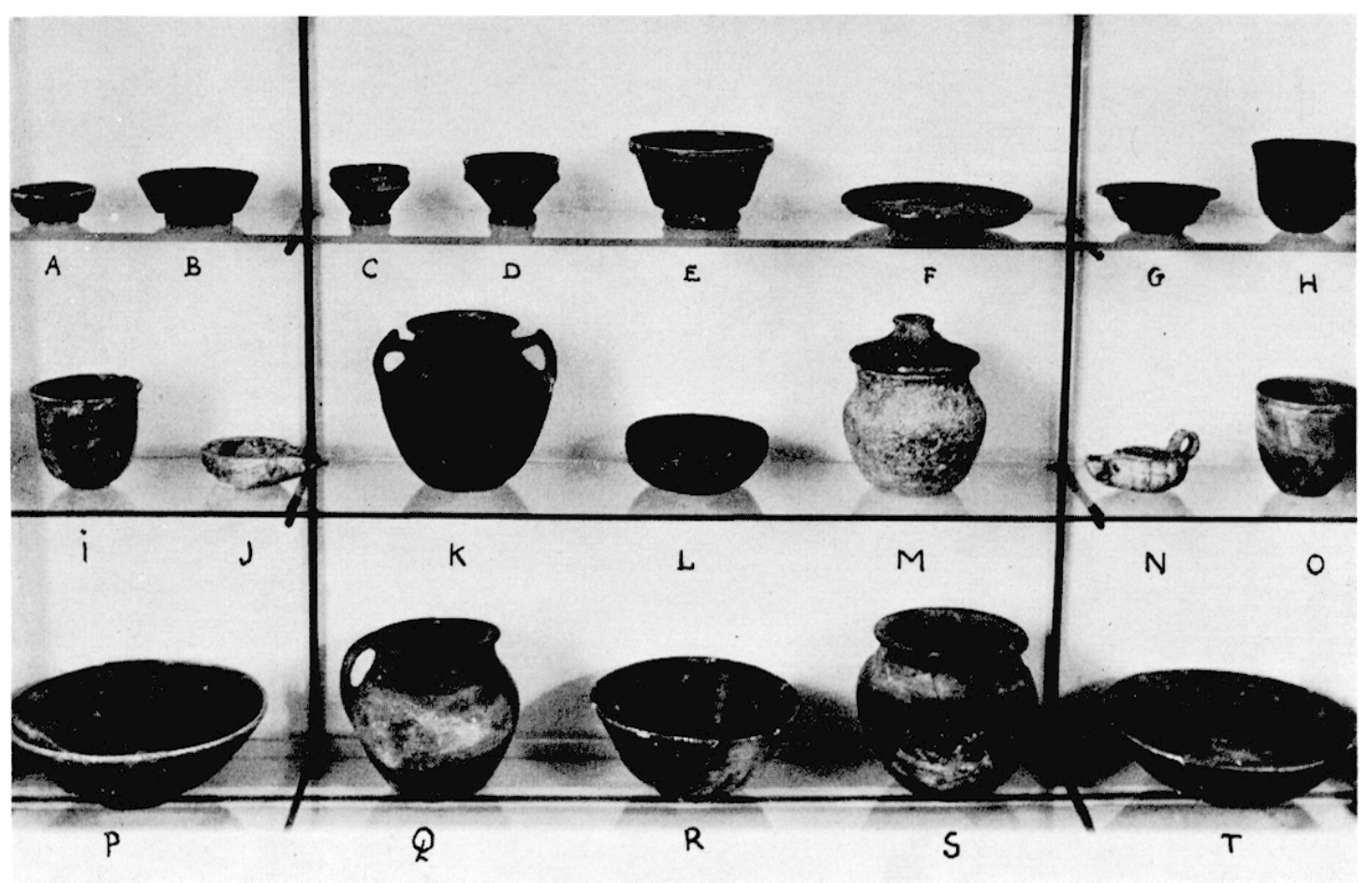

85. Vitrine de la fosse-silo $n^{\circ} 3$, an .Musie de (anvaillon.

IX. Fer. Lne rinquantaine de fragments de tiges et clous. ainsi que 2 plaquettes avec rivets.

X. Divers. Corne de chivre avec trace de sciage très marquée à la base, éclat de silex marron avec conchoïde de percussion présentant de fines retouches sur sa bordure. en 2 endroits. (fig. $76 \mathrm{~d}$ ).

XI. Vestiges organiques contenus dans la terre de la fosse $n^{0}: 3$.

A. Aliments d'origine végétale. $1^{0}$ Pain et galettes :

a) Fragments bien caractérisés d'un pain grossier de céréales. en forme arrondie de "miche", de taille movenne.

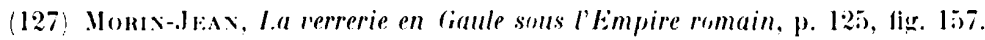


b) Fragments plus nombreux d'une sorte de galette plate, à la surface de laquelle apparaissent, ça et là, des pépins de raisin.

c) fragments d'une galette de même nature que la précédente avec pépins de figue.

20 Fruits et graines ${ }^{128}$ : Yoix (juglans sp.), figues (ficus sp.), pois chiche (cicer arielinum L), graines d'avoine (avena sp.), d'aubépine (azérolier ou aubépine du Roussillon : cralaegus ruscillonensis $L$ ), de courge (cucurbila sp.), de groseiller commun (ribes rubrum $L$ ) de pin-pignon (pinus pinea $L$ ), de paliure austral (paliurus australis - Rhoem et Sch.), de palmier nain (chamaerops humilis), noyaux de cerisier de Sainte-Lurie (cerasus Mahaleb) Mill.) et d'olives (olea europea $L$ ).

Cette nomenclature appelle quelques remarques. Yotons d'abord que les graines ne sont aucunement carbonisées; leur séjour en terre depuis environ ¿ millénaires n’en a pas altéré la forme. C'ne lente minéralisation s'est effectuée. Ce ne sont pas des graines fossiles, mais en voie de fossilisation ${ }^{129}$. I)'autre part, on peut classer ces vestiges d'origine végétale en 2 catégories : ceux provenant du ramassage et ceux ayant nécessité une préparation. Parmi les 1 ers, il n'est pas étonnant de trouver les baies de l'aubépine et du groseiller, les graines du pin pignon ${ }^{130}$, des noyaux du cerisier Mahaleb, les olives, les noix, et les figues, fruits de plantes communes dans la contrée. Les graines de courge étaient peut-être utilisées pour la fabrication l'une huile (on sait que leur teneur en matière oléagineuse peut aller de 38 à $5 \% \%{ }^{131}$.

L'avoine pouvait servir à confectionner des galettes ou des bouillies. Plus singulière est l'existence des fruits du paliure austral, plante médicinale dont les graines sont astringentes et expectorantes ${ }^{132}$, et du palmier nain qui est la seule espèce indigène de l'Europe méridionale ${ }^{133}$.

En ce qui concerne le pain et les galettes, la différence entre les deux est très marquée. L'examen au microscope des fragments de pain a permis de voir nettement des fibres de tissu ligneux minéralisé accompagnant les grains concassés des céréales, tandis que les galettes, qui ne présentent aucun alvéole ou soufllure, semblent avoir été faites d'une pâte cuite après malaxage à l'eau sans levain. La présence de pépins de raisin et de figue parsemant leur surface est un témoignage de la pratique qu'avaient les anciens d'incorporer à

(128) Détermination effectuée par le professeur R. Salgues, de l'Institut botanique de Briqnoles (Var).

(129) "La matière organique ne s'est pas imprégnée de sels calcaires ou de silice, ou des deux, par apport de l'extérieur ", nous a précisé le professeur Salgues "elle s'est simplement épuisée en ses constituants labiles tels que protides, lipides et hydrates de carbone, ce qui l'a enrichie, si l'on peut dire, en cellulose et en matières minérales par le phénomène de concentration".

(130) J.es pignons de pommes de pin confits dans le miel, s'appellent chez les Taurins, acquiceles (PliNe, Hisl. nat., XV, 9).

(131) Dr A. Macrizio, Iisloire de l'alimenlation végélale depuis la Préhistoire jusqu'à nos jours (traduit par le Dr F. Givox), Payot, Paris, 1932, p. 313.

(132) Gildet et Magive, Nouvelle Rore française. Iescriplion des planles... avec l'indicalion de leurs propriétés et de leurs usages en médecine, $6^{\circ}$ édition, Paris, Garnier, 1887, p. 101. D'après Pl..Ne (Hist. Nal., LXXIV, 71 ), la graine du paliure ètait employée efficacement chez les Romains contre la piqùre des scorpions et aussi contre la maladie de la pierre et contre la toux.

(133) Le Maott et Decaisse, Trailé général de Bolanique descriplive el analylique, Paris, Didot, 1876, p. 653 et 636 : "palmier abondamment répandu en Sicile en Italie et en Espagne et qui peut vivre en plein air dans le Sud-Est de la France ". PLIX: (XIII, 9) indique que sa feuille est tris utile pour faire des liens. 
certaines galettes des condiments, épices ou autres ${ }^{134}$. Les Italiens dans certaines régions, font encore gonfler des raisins secs dans une pâte de céréales et les cuisent avec elle ${ }^{135}$.

B. Aliments d'origine animale et vestiges d'animaux utilisés dans l'alimentation. (Euf de pigeon, écailles et vertèbres de poisson, os de volaille, d'un oiseau de petite taille, de porc, mouton, bocuf, carapace de tortue terrestre (partie ventrale), hélix.

C. Animaux divers : cloportes, os de rongeurs.

D. Débris ligneux. Plusieurs fragments de tiges de bois, épaisses (frêne) dont la forme fait penser aux barreaux d'une échelle, ainsi qu'une coupelle creusée dans du bois de prunier ${ }^{136}$.

Cette fosse alimentaire contient un intéressant matériel archéologique de la $2^{\mathrm{e}}$ moitié du $\mathrm{I}^{\mathrm{er}}$ siècle av. J.-C., avec couche terminale d'époque Julio-Claudienne.

\section{Les fosses funéraires}

Trois sépultures à inhumation ont été découvertes dans la zone des puits. En arrachant des oliviers à la partie supérieure de son exploitation, en 1936, M. Jeune, propriétaire de la carrière, mit au jour 2 fosses peu profondes creusées dans le gravier à quelques mètres l'une l'une de l'autre. Tout ce que nous avons pu savoir, c'est qu'elles avaient la forme d'une "poche» et contenaient des tessons de poterie avec quelques os humains très fragmentés, qui ne furent malheureusement pas conservés, sauf une machoire incomplète de la l re fosse.

\section{FOSSE FLNÉRAIRE: $\mathrm{N}^{0}$ I}

I. Polerie fine. A. Céramique d'Asie mineure : 29 tessons que l'on peut classer ainsi d'après la composition de la pàte et la technique.

10. Bucchero gris éolien, de Villard ${ }^{137}$. Irgile gris clair très dure a engobe gris foncé très adhérent: fragment de bord oblique de large récipient avec traits ondés sous une rainure horizontale (fig. $86 \mathrm{a}$ ). tesson orné de lignes sinueuses sous des pointillés verticaux (fig. 86 b), fragment épais à 6 sillons parallèles (fig. $86 \mathrm{c}$ ), fragment de bord de vase caréné (fig. $86 \mathrm{~d}$ ).

$2^{0}$ Imitation massaliote du bucchero gris éolien.

a) Argile gris clair semi-dure, à engobe gris foncé, peu adhérent : 3 fragment de bord de bols carénés (fig. 86 , e f g) ${ }^{138}$; coupe incomplète à bord court oblique, et anse horizontale relevée ${ }^{139}$ (fig. $86 \mathrm{~h}$ ), pied profond oblique de grande coupe (fig. $86 \mathrm{i}$ ), 2 fragment de bords concaves (fig. $86 \mathrm{j} \mathrm{k}$ ). un fond plat (fig. 86 1), et 8 tessons.

b) Argile gris clair tendre, à engobe peu apparent : fragment de paroi de vase épais orné de lignes ondées très serrées (fig. $86 \mathrm{~m}$ ) et 7 tessons.

(134) Dr A. Mavaizio, op. cil., p. 515 et suiv.

(135) Id., ibid., p. 523. Au Musée d'art et d'archéologie de Clermond-Ferrand, dans une vitrine contenant la sépulture d'un jeune enfant provenant des Martres-de-Veyres (P.-de-D.), on peut voir une sorte de galette avec pépins de raisin, identique à celle de Cavaillon.

(136) Déterminations du laboratoire d'analyse du .lusée du Louve (Sept. 1959).

(137) F. Vil.tars, op. cil., p. 51 et 52.

(138) Id., ibid., pl. 7, nos 9, 10, 11.

(139) Id., ibid., a rapprocher des profils, pl.155, no 4 et pl.156, nol3. 


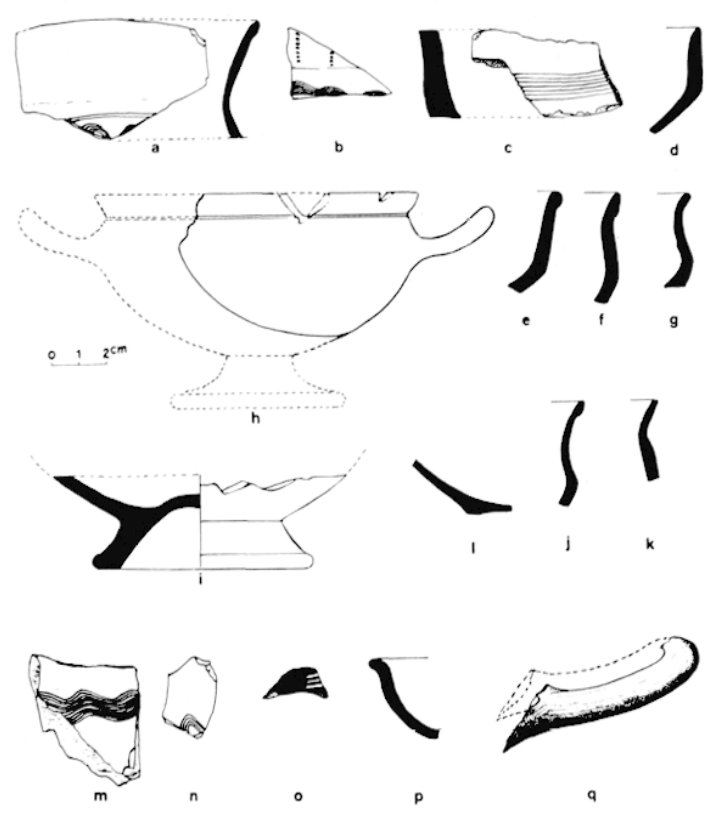

86. Fosse funéraire no 1 . Céramique phocéenne et imitation.

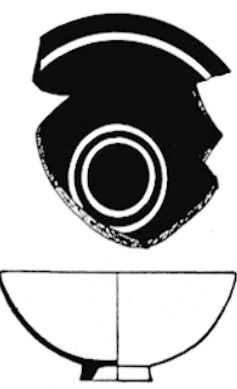

a
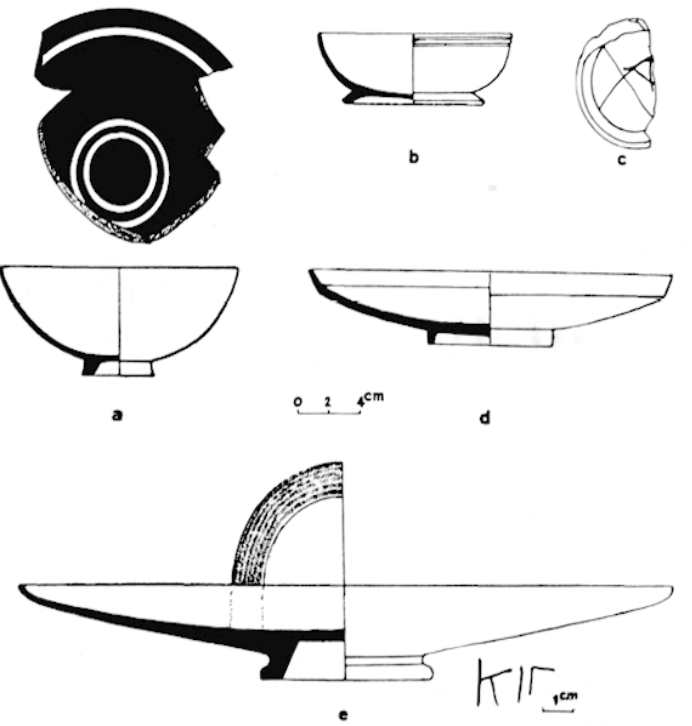
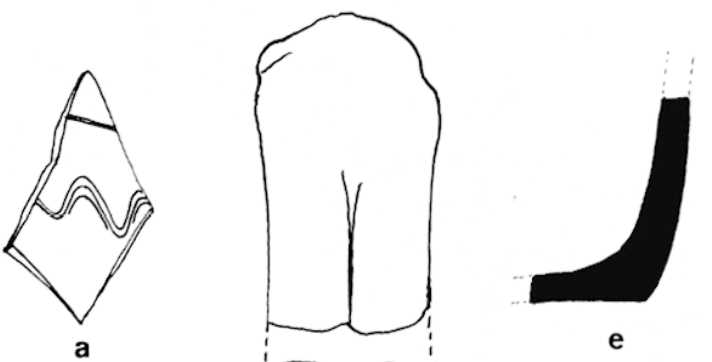

e
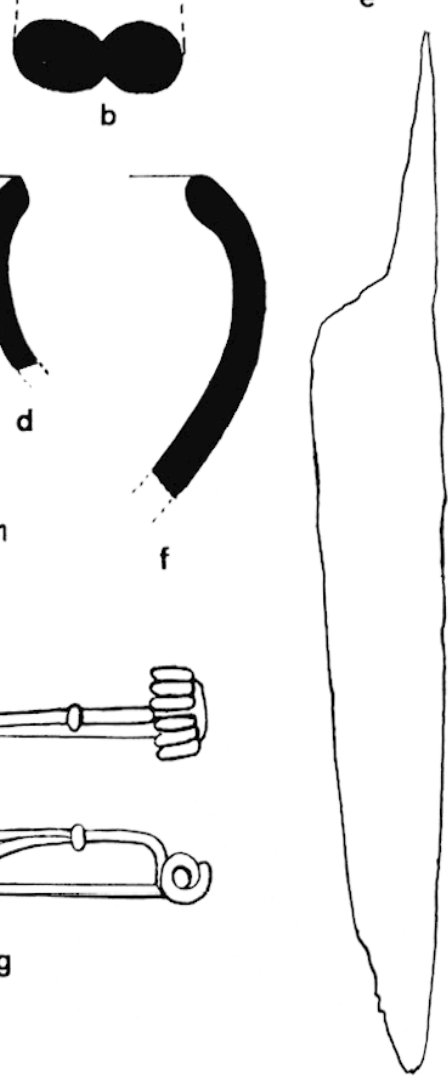

$\mathrm{h}$

87. Fosse funéraire no 2. Ceramique, fibule et couteau en fer.

c) Argile gris clair dure à engobe gris marron : fragment de bord de coupe carénée lisse ${ }^{140}$.

d) Argile gris clair dure sans engobe : petit fragment orné de traits ondés (fig. $86 \mathrm{n}$ ).

B. Céramique "attique ", italiote : un seul petit tesson en argile pâle, à vernis noir brillant, avec 3 bandes peintes en rouge (fig. 86 o).

II. Polerie commune. Fragment de coupe en argile rougeâtre lissée, à surface marron mate (bord un peu déversé) (fig. $86 \mathrm{p}$ ), anse incomplète latérale de coupe imitation indigène du type gris éolien, en terre cuite jaune clair lustrée (fig. 86 q).

(140) Id. ibid.. bucchero gris local, pl. 36, no 5 et pl. 37, no 4. 
III. Mâchoire humaine. Branche inférieure gauche d'une màchoire humaine qui se distingue par la hauteur et le peu de longueur du maxillaire. La hauteur de la branche horizontale, prise au niveau des prémolaires, est de $3,9 \mathrm{~cm}$, sa longueur de $8 \mathrm{~cm}$. Les dents régulières el saines semblent appartenir à un homme dans la force de l'àge. I noter un intervalle assez grand entre la dernière molaire et la branche montante du maxillaire.

\section{FOSSE FLNÉRAIRE $\mathrm{N}^{0} 2$}

I. Céramique. A. Polerie fine. fragment de paroi de vase en argile grise. dure, à engobe foncé (bucchero gris, de Villard) orné d'une double ligne sinueuse (fig. 87 a), anse double avec traces d'engobe (fig. $87 \mathrm{~b}$ ), fragment de coupe en Campanien B (fig. $87 \mathrm{c}$ ).

B. Polerie commune. fragment de bord évasé et fond d'olla de type hallstattien (fig. $87 \mathrm{~d} \mathrm{e}$ ), de couvercle d'urne, et de jatte à paroi courbe et bord rentrant (fig. $87 \mathrm{f}$ ).

II. Fer. Fibule (long : $8 \mathrm{~cm}$,), lype caractéristique de la Tène II à appendice caudal orné d'un bouton globuleux réuni au milieu de l'arc par une bague épaisse annulaire, ressort bilatéral à 6 spires a corde extérieure (fig. $87 \mathrm{~g}$ ). Couteau en fer à lame triangulaire allongée, avec soie pointue dan: le prolongement d'un des bords (long : $18 \mathrm{~cm}$ ) (fig. $87 \mathrm{~h}$ ).

\section{FOSSE FLNÉRAIRE xo 3}

Découverte en 1954 dans la carrière Bouchet, clle se présentait creusée dans le gravior sur $3 \mathrm{~m}$. de haut el $1.30 \mathrm{~m}$. de large. Des fouilleurs clandestins ont récupéré le matériel archéologique qu'elle contenait et nous l'ont remis quelques temps après. Cette $3^{\mathrm{e}}$ fossc sépulcrale est très différentr des 2 premières : clle se classe à la mème époque que celle du romblement des puits ot fosses-silos, (2e moitié du Ier siècle av. .J.-C.).

I. Céramique. Campanien 1 : 1. Patère f. 7, avec 4 trous de réparation, dont l'un est encore obstrué par du plomb, le long d'une cassure ayant intéressé tout le diamètre du récipient (H. : $4.8 \mathrm{~cm}$. I). (). : 24.3 cm.) (fig. $88 \mathrm{~d}$ ).

2. Bol haut (f. :31), avec 3 bandes peintes intérieures, l'une pres du bord, les 2 autres sur le fond (H. : $7,1 \mathrm{~cm}$. D. (). : 15,4 cm.) (fig. 88 a).

3. Pied de vase avec graflite à la partie exlerne (fig. 88 c) .

Campanien B : 1. Grand plateau (f. 5) à pied profond oblique, guilloché intérieurement. et bords peu élevés, arrondis. (irafrite près du pied à l'extérieur (II. : $5,6 \mathrm{~cm}$. D. 0. : $42.8 \mathrm{~cm}$.) (fig. 88 e) et fragment d'un autre semblable.

2. Bol à pied tres large. bord a 2 sillons parallèles (forme I) (H. : 4.5 cm. D. (). : 12.6 cm.) (fig. $88 \mathrm{~b}$ ).

11. Lampe. Lampe en campanien (: (engobe gris), à bec évasé arqué à son extrémité. stries lombantes tout autour d'une cuvette rétrécie, anse épaisse annulaire. Sous le réservoir lettre $L$ (II. : :3.4 cm. L. : $10.3 \mathrm{~cm}$.) (fig. 89 j). ('est le type de lampe funéraire des tombes de saint-Rémy-deProvence ${ }^{14}$. . Ion figurée par Dressel, cette forme paraît être contemporaine du type delphinoïde (époque républicaine).

III. Monnaie. Petit bronze coulé, très boursouflé, imitation barbare de la pièce au taureau de Massalia. A l'avers. tète à gauche. au revers, taureau ì gauche (fig. 89 k).

(141) P. de Brix, Nole sur quelques sépullures gallo-grerques..., "p. cil., p1. 1, 10 10. Idem, l.es lampes antiques en argile du . Musee des Alpilles..., "p. cil., p. $3,1^{\circ} 2$. 

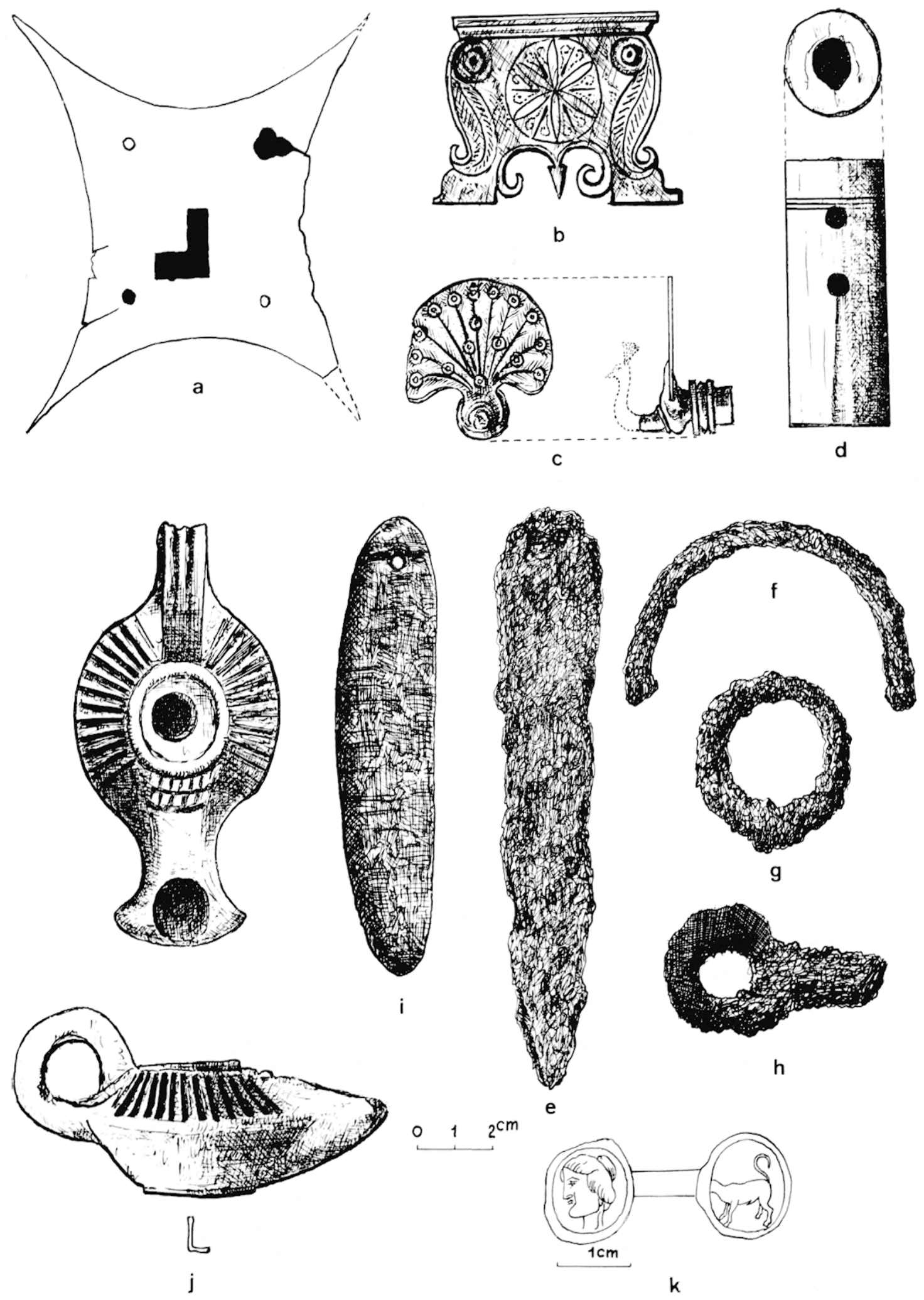

89. Fosse funéraire no 3 . Mobilier. 
IV. Bronze. Éléments d'un cofiret comprenant :

10 Une plaque d'entrée de serrure, mince, quadrangulaire, à pointes allongées, avec trous de fixation et fente coudée pour l'insertion de la clef (fig. 89 a);

$2^{\circ}$ Lne plaque ciselée à décor gravé au burin sur une face. Le motif central représente une fleur à 6 pétales allongés avec volutes affrontées en pointillé entre les pétales, le tout inscrit dans un cercle. De chaque còté : feuille nervurée courbe à enroulement lerminal (fig. 89 b) ;

$3^{0}$ Autre plaque plus petite représentant un paon faisant la roue, formant corps avec une tige moulurée (fig. $89 \mathrm{c}$ ).

V. Fer. Lame de grand couteau ou poignard, très oxydée (L. : $14 \mathrm{~cm}$ ) (fig. $89 \mathrm{e}$ ), anse en arc de cercle (fig. 89 f), grand anneau fermé (fig. $89 \mathrm{~g}$ ), tige courte épaisse terminée par un anneau à la surface duquel on remarque les restes d'un tissu à fils entrecroisés imprégnés d'oxyde de fer (fig. 89 h).

VI. (Os. 8 éléments de charnière cylindriques, à trous latéraux (fig. $89 \mathrm{~d}$ ), qui faisaient partie sans doute du colfret décrit plus haut.

VII. P'ierre. galet allongé rappelant le brunissoir néolithique, en roche dure vert foncé. perforé a une exlrémilé et portant sur sa surface des stries dorées avec traces ferrugineuses (fig. $89 \mathrm{i}$ ).

VIIJ. Ossements humains. Du squelette qui se trouvait à l'intérieur, seuls ont été conservés quelques fragments de boîle crànienne.

\section{Addendum}

Il nous faul enfin mentionner quelques tessons de poterie grecque, découverts en juillet 195i). lors du creusement d'une piscine dans le terrain de M. Miller, chirurgien-dentiste, confrontant à l'Est la carrière Bouchel.

10 Partie centrale, avec cupule profonde (diam. : 5 cm) d'une "assielte à poisson " en poterie campanienne à pàte.$^{142}$. forme 2.3 de Lamboglia (fig. 90 a) ( $1 v^{\mathrm{e}}-11 \mathrm{I}^{\mathrm{e}}$ s. av. J.-(C.). I noter un graffite sur la paroi externe en 2 endroits et des empreintes digitales particulierement nettes sur le bord du pied.

$2^{\circ} 2$ vases incomplets a haut bord vertical munis de 2 anses latérales dont il ne reste que le: allaches (fig. 90 b). L'un est en argile pàle à vernis noir externe, passant au marron foncé à l'intérieur qui porte des traces de hachures; l'autre est à vernis noir métallique par places à fond orné de palmettes dont on ne voit que le sommet.

$3^{\circ}$ Fragment de fond de vase avec palmette sous une rangée de petits traits doubles (fig. 90 c).

$$
\therefore
$$

La découverte de puits et fosses antiques sur les pentes terminales de la colline SaintJacques, à Ciavaillon, au cours de l'exploitation de carrières de gravier, apporte une appréciable contribution à l'archéologie de l'ancienne cité des Cavares, en mettant en relief un aspect nouveau de sa civilisation et en élargissant considérablement les connaissances de

(142) Sur cette forme particulière de récipient, voir F. BexorT, l.e Grand Congloué, p. 84 et p. 87, pl. VIII. 67 exemplaires ont eté trouves dans l'épave du navire grec explorée par l'équipe du Commandant Cousteau. Cf. aussi M. Almagro, op. cil., I, tombe a incinération, Las Corts, $n^{\circ} 113$, p. 356, fig. 339, $n^{\circ} 6$, mais la caviti centrale est plutót petite et conique. 


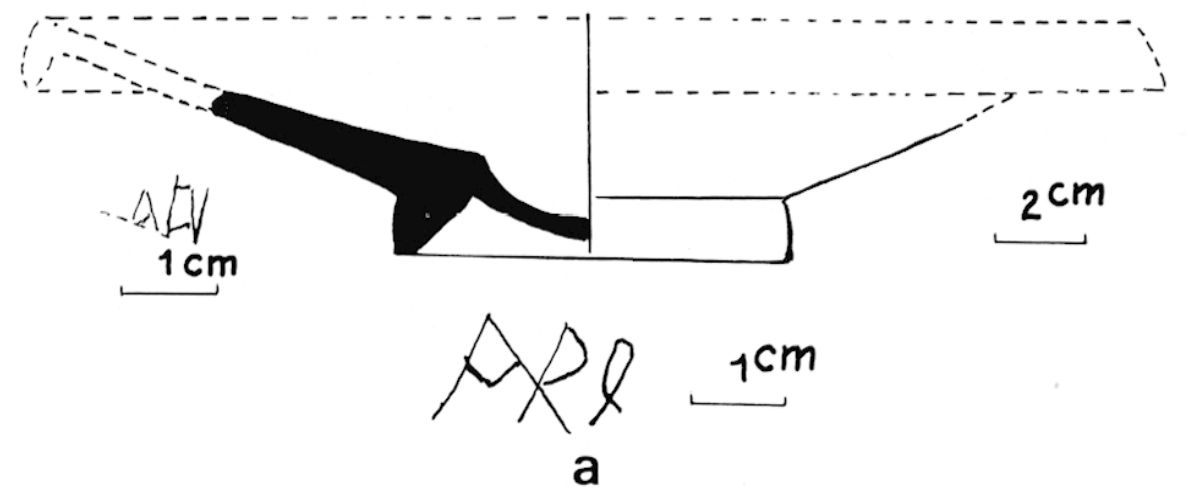

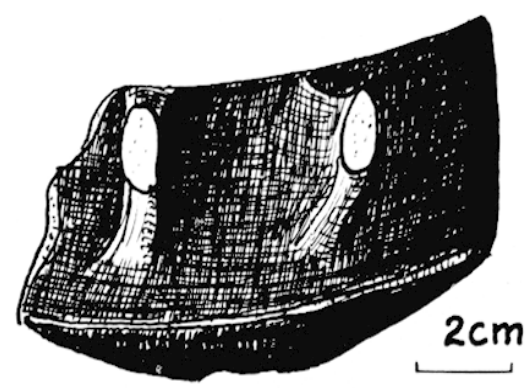

b

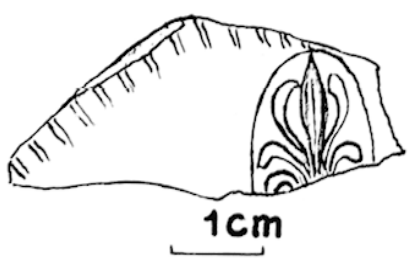

C

90. Ciramique campanienne du terrain Miller.

la céramique en usage chez ses habitants. La présence de ces excavations au contact de l'oppidum et de la plaine montre qu'avant l'arrivée des Romains, les populations primitives n'étaient pas exclusivement cantonnées sur le sommet de la colline, à l'intérieur du périmètre des remparts, mais qu'elles s'étendaient en dehors du camp retranché près des terres cultivables où s'est développé dès la fin du 1er Age du Fer, un habitat rural important au voisinage d'une voie antique ${ }^{143}$. En cas de danger, l'oppidum tout proche servait de refuge. Les puits ne sont pas, bien entendu, localisés seulement aux endroits où les carrières ont été ouvertes ; leur emplacement en surface se signale à l'attention par une végétation plus haute, plus touffue et de forme circulaire.

Quoique n'étant pas uniformément répartis et autant que l'on puisse en juger par un examen superficiel, ils paraissent disséminés dans le même secteur sur environ 3 hectares.

Il faut noter que la colline Saint-Jacques ne possède aucune source. Les besoins en eau des occupants de l'oppidum étaient assurés, comme le sont encore ceux des propriétaires des "bastides", par des citernes taillées dans le rocher (on en voit encore en plusieurs en-

(143) Les stèles funéraires celto-grecques étudiées par Félix Mazacric: (Noles sur une imporlanle découverle d'inscriplions celliques, dans Revue du Midi, Nìmes, 1910), ont été trouvées dans le territoire de Cavaillon à environ $2 \mathrm{~km}$. de la colline Saint-Jacques. l. auteur avait conclu que ces stèles provenaient du quartier de Saint-Bardou (ou des Vergers), oủ passait l'ancienne voie sallo-grecque longeant là le pied de la colline (à proximité des puits). 
droits). Dans les parties basses de la colline, l'existence d'une nappe d'eau souterraine peu profonde et la facilité de creuser le gravier, ont donné lieu au forage de nombreux puits à une époque qui ne doit pas remonter plus haut que la fin du 1er Age du Fer. Auprès de ces puits, se sont groupées, vers le ve siècle av. J.-C., des habitations, d'abord simples cabanes indigènes en matériaux périssables, dont toute trace a disparu, puis des constructions plus importantes, de technique gallo-romaine, au milieu du ier siècle av. J.-C. possédant. des silos ou garde-manger pour conserver les denrées.

En ce qui concerne les puits, si donc, à l'origine, ils ont été bien creusés pour avoir de l'eau, on peut se demander quelle a été la raison de leur comblement ? Remarquons d'abord qu'un certain nombre d'entre eux (4 pour 10 environ) sont restés vides, dans lesquels on a par conséquent continué à puiser de l'eau, ce qui semblerait indiquer qu'il n'y a pas eu abandon général de ces puits, mais plutôt une réduction dans le nombre de leurs usagers. Ce fait peut s'expliquer par une mise en culture d'une partie des terrains jusqu'alors en friche; il en a résulté la disparition de l'aire des habitations primitives et la fin de l'utilisation d'un certain nombre de puits qui ont alors servi de dépotoir ${ }^{144}$. Quant aux fosses à provisions, qui sont à classer dans le "gallo-romain précoce», elles ont cessé d'exister comme telles pour la même raison que les puits, c'est-à-dire par l'abandon des habitations dont elles lépendaient, leur remplissage terminal étant le fait de ceux qui continuaient à vivre sur le site ou aux alentours. Elles ont l'avantage d'avoir conservé des vestiges de leur première destination : échantillons des denrées qui y étaient entreposées mêlées aux vases brisés qui les contenaient.

Mis à part les puits no 1 et 13 qui ont été comblés à la fin du ier Age du Fer, le début de l'utilisation de ces excavations comme dépotoir peut se situer vers le milieu du I $^{\mathrm{er}}$ siècle. av. J.-C. Leur abandon final est daté par les poteries les plus récentes des puits no 4 , 5) et 6 : sigillée de la Graufesenque tardive et des ateliers de l'Est, et lampes de l'époque des Antonins, soit la fin du II $^{\mathrm{e}}$ siècle ap. J.-(:.

Les fosses sépulcrales à inhumation ne se rattachent à aucun ensemble funéraire ${ }^{145}$. La fosse $n^{0} 1$ est la plus ancienne, probablement du ve siècle av. J.-C. avec de la poterie grise d'Asie Mineure tardive ; la fosse $\mathrm{n}^{0} 2$ a donné une fibule de la Tène II ; la fosse $n^{0} 3$ est du milieu du $\mathrm{I}^{\mathrm{er}}$ siècle av. J.-C.

L'examen du contenu des puits et fosses suggère quelques remarques :

10 Importance de la céramique d'origine massaliote, que ce soit l'imitation attardée de la "phocéenne " grise ( $v^{e}$ siècle av. J.-C. avec fibules de la Tène I dans le puits no 13 ) ou celle de la Campanienne à vernis noir ou gris du I $^{\mathrm{er}}$ siècle av. J.-Ci. Ceci n'a rien de surprenant, les relations commerciales entre la métropole grecque et l'agglomération cavare (qu'Artémidore qualifiait de "ville de Marseille ") étant attestée par la grande quantité de pièces de monnaie de Massalia trouvées sur l'oppidum depuis les oboles au revers en

(144) Des puits semblables ,ayant servi de dépotoir après leur destination primitive, ont été signalés en de nombreux endroits. Citons seulement ceux d'Alésia, où l'on avait "précipité tous les résidus de la vie courante * (J. Toutaix, op. cil., p. 161), ceux de l'oppidum des Nitiobriges (Agen), du Crêt-Châtelard etc. (J. Dŕchelertr, dans Bullelin monumental, 1903 , p. 562 et 563).

(145) La région de Cavaillon a fourni un seul puits sépulcral mais il est de l'époque néolithique : $\mathrm{S}$. Gagisiens: et L. Vareild.es, Puits funéraire de Couslellel à Cabrières d'Avignon (Vse), Cahiers d'IIisl. el d'Arch. de Nìmes, $n^{\cap} 3$. 
creux de haute époque jusqu'aux pièces de la décadence, et aussi par l'existence d'un petit bronze portant la légende : KABE, en caractères grecs.

20 La poteric indigène se divise en deux catégories nettement différenciées :

a) des formes hallstattiennes attardées : urnes non tournées à décor caractéristique au col et ouverture à lèvres évasées, gobelets et écuelles lissées, dont certaines de fabrication soignée (puits nos 1 et 13 ).

b) des types de la Tène, dans la majorité des autres : urnes tournées, à rebord horizontal et encoches au col, avec couvercles, jattes à goulot court et anses en ruban aplati, plateaux circulaires, marmites, bassines, toujours accompagnés par des vases à liquide en terre jaune dont les plus caractéristiques sont les pichets à anse torsadée, les "olpi " à panse globuleuse et les mortiers à bec de déversement et emplacements digitaux pour la préhension.

$3^{\circ}$ La céramique d'importation est caractérisée par l'abondance des petits gobelets à "parois minces ", la qualité des vases arétins et la rareté des coupes à "décor oculé ", de l'Ihérique et de la poterie peinte de Lezoux.

Retenons l'importance des vestiges datant de la deuxième moitié du I ${ }^{\mathrm{er}}$ siècle av. J.-C., principalement au début de l'Empire romain. C'est précisément la période où la cité de Cabellio, fondée par les conquérants au pied de la colline, venait de se voir attribuer le titre de colonie latine par Auguste, et frappait, sous le XIe consulat de cet empereur ( 23 av. J. (i.) des monnaies autonomes avec la légende : $C O L$. CABE. au revers de la corne d'abondance, ouvrant une ère de prospérité qui ne s'est pas démentie au cours des siècles et fait encore aujourl'hui sa renommée.

André [)emollis. 